\title{
DIGITAL MODEL OF GROUND-WATER FLOW IN THE PICEANCE BASIN, RIO BLANCO AND GARFIELD COUNTIES, COLORADO
}

U.S. GEOLOGICAL SURVEY

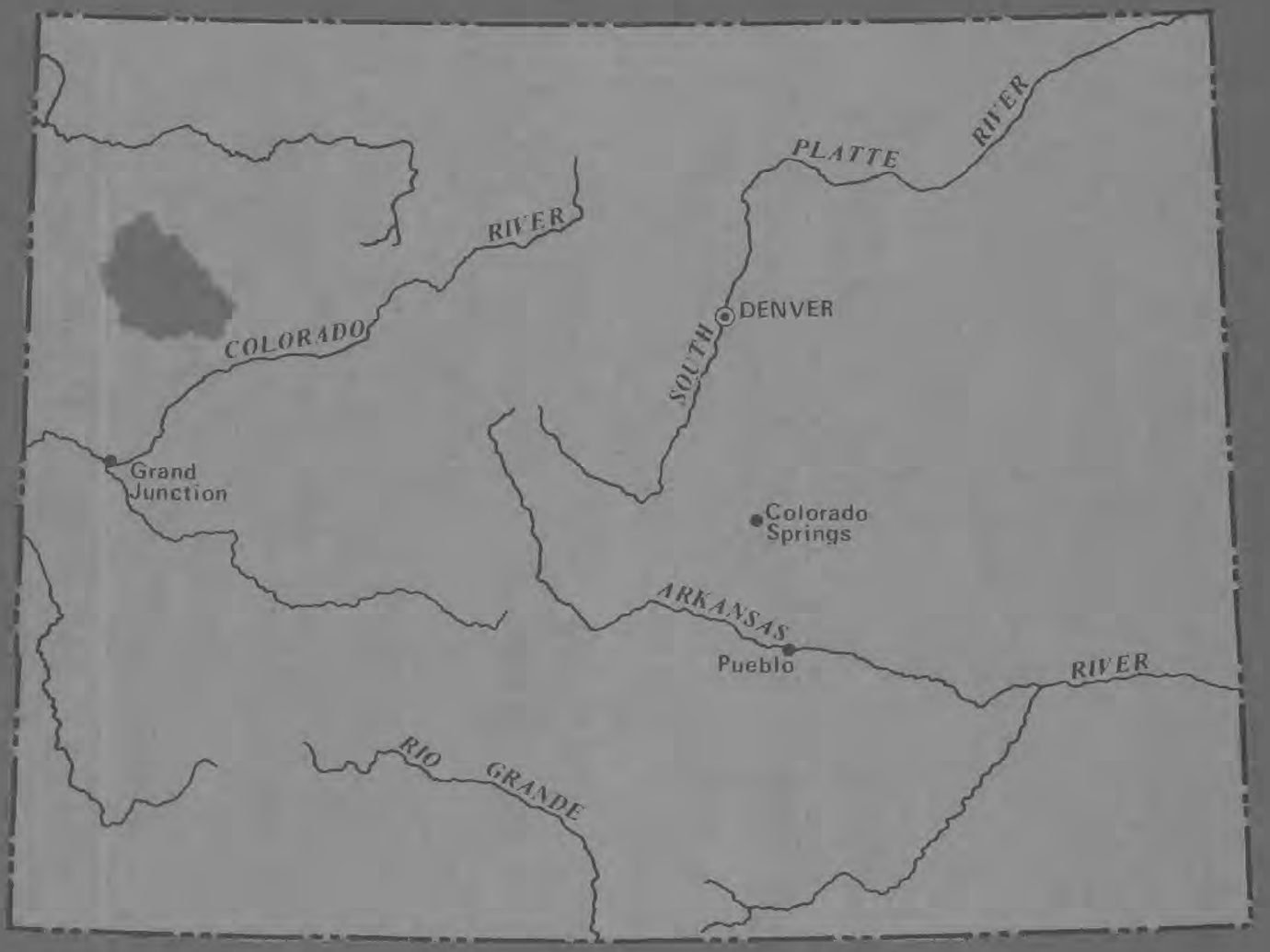

Water Resources Investigations $78-46$ 


\begin{tabular}{|c|c|c|}
\hline $\begin{array}{l}\text { BIBLIOGRAPHIC DATA } \\
\text { SHEET }\end{array}$ & 1. Report No. & 3. Recipient's Accession No. \\
\hline \multirow{2}{*}{\multicolumn{2}{|c|}{$\begin{array}{l}\text { 4. Title and Subtitle } \\
\text { DIGITAL MODEL OF GROUND-WATER FLOW IN THE PICEANCE BASIN, } \\
\text { RIO BLANCO AND GARFIELD COUNTIES, COLORADO }\end{array}$}} & $\begin{array}{r}\text { 5. Report Date } \\
\text { June } 1978 \\
\end{array}$ \\
\hline & & 6. \\
\hline \multicolumn{2}{|l|}{$\begin{array}{l}\text { 7. Author(s) } \\
\text { John B. Weeks }\end{array}$} & $\begin{array}{l}\text { 8. Performing Organization Rept. } \\
\text { No. USGS/WRI } 78-46\end{array}$ \\
\hline \multicolumn{2}{|c|}{$\begin{array}{l}\text { 9. Performing Organization Name and Address } \\
\text { U.S. Geological Survey, Water Resources Division } \\
\text { Denver Federal Center, Box } 25046 \text {, Mail Stop } 415 \\
\text { Lakewood, Colorado } 80225\end{array}$} & $\begin{array}{l}\text { 10. Project/Task/Work Unit No. } \\
\text { 11. Contract/Grant No. }\end{array}$ \\
\hline \multirow{2}{*}{\multicolumn{2}{|c|}{$\begin{array}{l}\text { 12. Sponsoring Organization Name and Address } \\
\text { U.S. Geological Survey, Water Resources Division } \\
\text { Denver Federal Center, Box } 25046 \text {, Mail Stop } 415 \\
\text { Lakewood, Colorado } 80225\end{array}$}} & $\begin{array}{l}\text { 13. Type of Reporr \& Period } \\
\text { Covered } \\
\text { Final }\end{array}$ \\
\hline & & 14. \\
\hline
\end{tabular}

15. Supplementary Notes

16. Abstracts

The digital model used to simulate ground-water flow in the aquifer system in the basin drained by Piceance and Yellow Creeks in northwestern Colorado is described in detail. The model is quasi three-dimensional in that it simulates ground-water flow in a multiaquifer system by assuming horizontal flow in the aquifers and vertical flow through the confining layers separating the aquifers. The model uses the iterative alternating-direction implicit procedure to solve the finite-difference flow equations.

The digital model is documented by a program listing and flow charts. Data used in the model and sample output are presented to document the simulation of steady-state flow in the aquifer system. The variables used in the computer program and program options are discussed in detail.

17. Key Words and Document Analysis. 17a. Descriptors

Colorado, Computer models, Ground-water movement, 0il shale

17b. Identifiers/Open-Ended Terms

Piceance basin, Uinta Formation, Green River Formation

17. COSATI Field 'Group

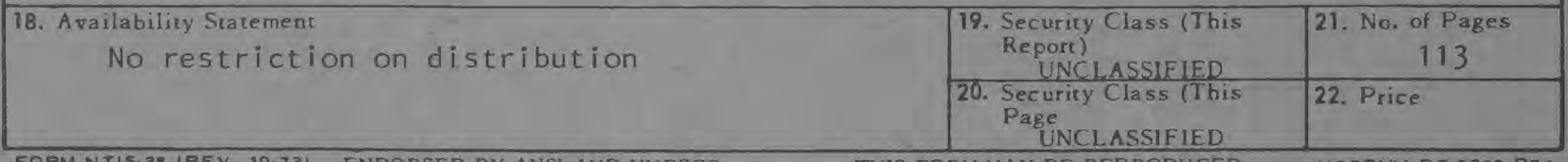

FORM NTIS.35 IREV. 10-73) ENDORSED BY ANSI AND UNESCO.

THIS FORM MAY BE REP RODUCED 
DIGITAL MODEL OF GROUND-WATER FLOW IN THE PICEANCE BASIN, RIO BLANCO AND GARFIELD COUNTIES, COLORADO

By John B. Weeks

U.S. GEOLOGICAL SURVEY

Water-Resources Investigations 78-46 


\title{
UNITED STATES DEPARTMENT OF THE INTERIOR
}

\author{
CECIL D. ANDRUS, Secretary \\ GEOLOGICAL SURVEY
}

H. William Menard, Director

For additional information write to:

District Chief

U.S. Geological Survey

Box 25046, Mail Stop 415

Denver Federal Center

Lakewood, Colo. 80225 
Metric conversions . . . . . . . . . . . . . . . . . . . . IV

Abstract . . . . . . . . . . . . . . . . . . . . . . . 1

Introduction . . . . . . . . . . . . . . . . . . . . . . 1

Purpose and scope . . . . . . . . . . . . . . . . . . . 3

Multiaquifer system . . . . . . . . . . . . . . . . . . . 3

Digital model. . . . . . . . . . . . . . . . . . . . . 5

Conceptual model. . . . . . . . . . . . . . . . . . . 6

Mathematical model. . . . . . . . . . . . . . . . . 8

Ground-water flow equation. . . . . . . . . . . . . . . 8

Finite-difference approximations. . . . . . . . . . . . . . . . 9

Model geometry. . . . . . . . . . . . . . . . . . . . 15

Computer program . . . . . . . . . . . . . . . . . . . . 15

Program variables . . . . . . . . . . . . . . . . . 15

Program options . . . . . . . . . . . . . . . . . . . . 18

Input variables... . . . . . . . . . . . . . . . . . 19

Ma in program. . . . . . . . . . . . . . . . . . . . 24

Subrout ine PARLOD... . . . . . . . . . . . . . . . . . 28

Subrout ine TRANS. . . . . . . . . . . . . . . . . . . 33

Subroutine BOUDY. . . . . . . . . . . . . . . . . . . . 39

Subrout ine RCHRG. . . . . . . . . . . . . . . . . . . . . 42

Subroutine ITERAT. . . . . . . . . . . . . . . . . . . 46

Subroutine COMPRC . . . . . . . . . . . . . . . . . . . 49

Subrout ine OUTPT. . . . . . . . . . . . . . . . . . . . 54

Subroutine CHCKP. . . . . . . . . . . . . . . . . . . 57

Subrout ine CHCKQ. . . . . . . . . . . . . . . . . . . . . 61

Input-data listing. . . . . . . . . . . . . . . . . . 63

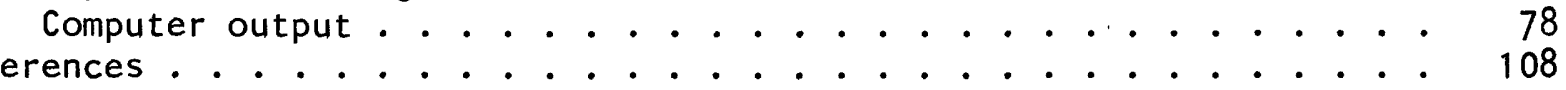

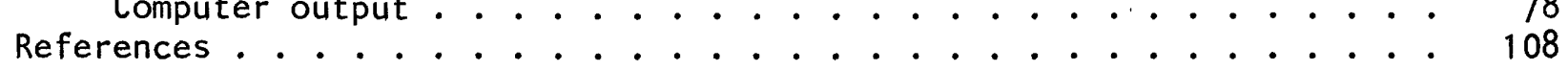

\section{ILLUSTRATIONS}

Figure 1. Map showing location of the Piceance basin and prototype

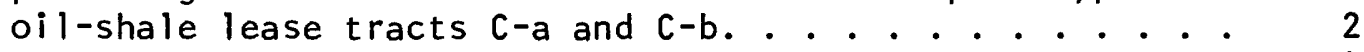

2. Geohydrologic section through the Piceance basin. . . . . . 4

3. Diagram showing flow model of the aquifer system. . . . . 6

4. Map showing aquifer boundaries used in the digital model of the ground-water system. . . . . . . . . . . . 7

5. Diagram showing index scheme for finite-difference grid at block $(i, j)$. . . . . . . . . . . . 10

6. Diagram showing block-centered, finite-difference grid used to model the Piceance basin aquifer system 


\section{TABLES}

Table 1. Definitions of variables used in the computer program which

2. FORMATs for input variables read by the computer program. . . 20

\section{METRIC CONVERSIONS}

The U.S. customary units used in this report can be converted to metric units by multiplying by the factors given in the following table:

To convert U.S. customary unit

foot

gallon

mi le

square mile
Multiply by

0.3048

3.785

1.609

2.590
To obtain metric unit

meter

liter

kilometer

square kilometer 
DIGITAL MODEL OF GROUND-WATER FLOW IN THE PICEANCE BASIN, RIO BLANCO AND GARFIELD COUNTIES, COLORADO

By John B. Weeks

\section{ABSTRACT}

The digital model used to simulate ground-water flow in the aquifer system in the basin drained by Piceance and Yellow Creeks in northwestern colorado is described in detail. The model is quasi three-dimensional in that it simulates ground-water flow in a multiaquifer system by assuming horizontal flow in the aquifers and vertical flow through the confining layers separating the aquifers. The model uses the iterative alternating-direction implicit procedure to solve the finite-difference flow equations.

The digital model is documented by a program listing and flow charts. Data used in the model and sample output are presented to document the simulation of steady-state flow in the aquifer system. The variables used in the computer program and program options are discussed in detail.

\section{INTRODUCTION}

The Piceance basin (fig. 1), which consists of the drainage basins of Piceance and Yellow Creeks, contains extensive deposits of oil shale in the Green River Formation of Eocene age (Donnell, 1961). The hydrology of the Piceance basin has been studied by the U.S. Geological Survey since 1964, when the Survey, in cooperation with the Colorado Water Conservation Board, began a reconnaissance investigation. Coffin, Welder, Glanzman, and Dutton (1968) and Coffin, Welder, and Glanzman (1971) reported on the study.

Intensive environmental studies have been conducted in the area since 1971 when the U.S. Department of the Interior announced plans for a prototype leasing program to permit development of a small part of the oil-shale resources on public lands in Colorado, Utah, and Wyoming. In 1974, two prototype leases in the Piceance basin, tracts $\mathrm{C}-\mathrm{a}$ and $\mathrm{C}-\mathrm{b}$, were sold by competitive bidding for private development under controlled conditions. Development of lease tracts $c-a$ and $c-b$ will require mine-dewatering systems which may have significant effects on the water resources of the Piceance basin. 


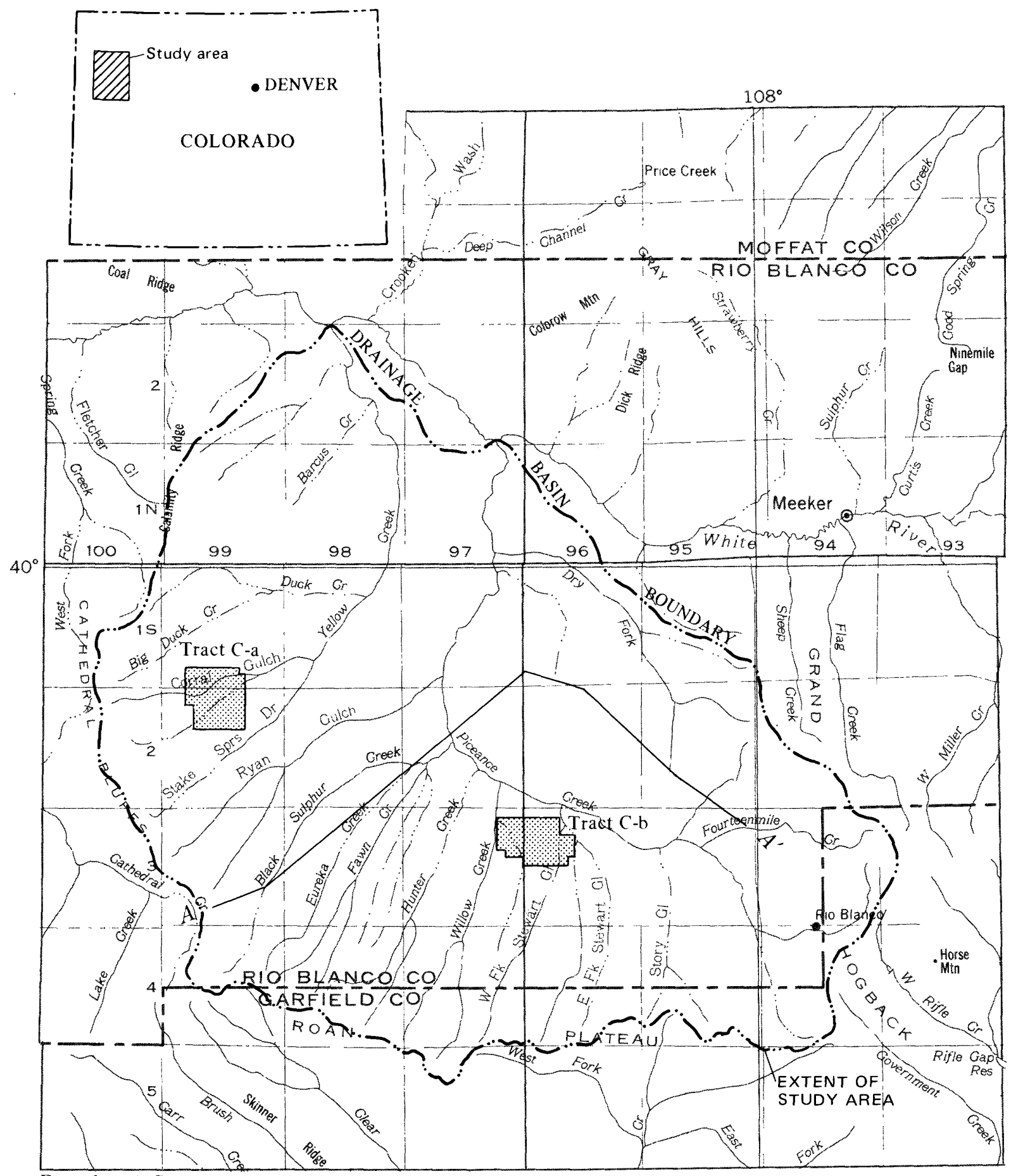

Base from U.S. Geological Survey

State base map, 1969

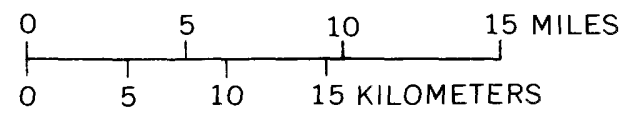

Figure 1.-- Location of the Piceance basin and prototype oil-shale lease tracts $\mathrm{C}-\mathrm{a}$ and $\mathrm{C}-\mathrm{b}$. (Modified from Weeks and others, 1974.) 
In 1972, the U.S. Geological Survey, in cooperation with the Colorado Department of Natural Resources, began a comprehensive study of the water resources of the Piceance basin. Water-resources data were compiled and published by Ficke, Weeks, and Welder (1974) and Weeks and Welder (1974). A water budget for Piceance and Yellow Creeks drainage basins was developed by Wymore (1974) and an evaluation of hillslope and channel erosion was made by Frickel, Shown, and Patton (1975). Ground-water flow and surface-water runoff models were developed and used to predict the impact of development on the quantity and quality of water in the basin. The results of the study were published in "Simulated Effects of Oil-Shale Development on the Hydrology of Piceance Basin, Colorado," by Weeks, Leavesley, Welder, and Saulnier (1974), U.S. Geological Survey Professional Paper 908.

\section{Purpose and Scope}

The report by Weeks, Leavesley, Welder, and Saulnier (1974) briefly describes the digital model used to simulate ground-water flow in the Piceance basin; however, details of the model were not presented. The purpose of this report is to describe the ground-water flow model in detail and document the computer program used to make the analyses contained in the above report.

The digital model was designed to simulate flow in the multiaquifer system in the Piceance basin. The theoretical development of the finitedifference equations used to approximate the equations governing ground-water flow and a complete listing of the computer program used to solve the equations are included in this report. Input data used in the model and the resulting solution output are presented also. Although the model is specifically for the Piceance basin, those people who are experienced in modeling should have no difficulty in revising the computer program to simulate other multiaquifer systems.

\section{Multiaquifer System}

A brief description of the geohydrology of the Piceance basin is presented below. The purpose of this discussion is to familiarize the reader with the type of multiaquifer system simulated by the digital model. For a detailed discussion of the multiaquifer system, the reader is referred to Weeks, Leavesley, Welder, and Saulnier (1974).

The ground-water system in the Piceance basin, an area of about 900 square miles, consists of two principal aquifers. The upper and lower aquifers are separated by the Mahogany zone in the Parachute Creek Member of the Green River Formation as shown on figure 2. The Mahogany zone is less permeable than the aquifers it separates.

The upper aquifer consists of fractured, lean oil shale (marlstone) in the upper part of the Parachute Creek Member above the Mahogany zone and the fractured marlstone, siltstone, and sandstone of the overlying Uinta Formation 


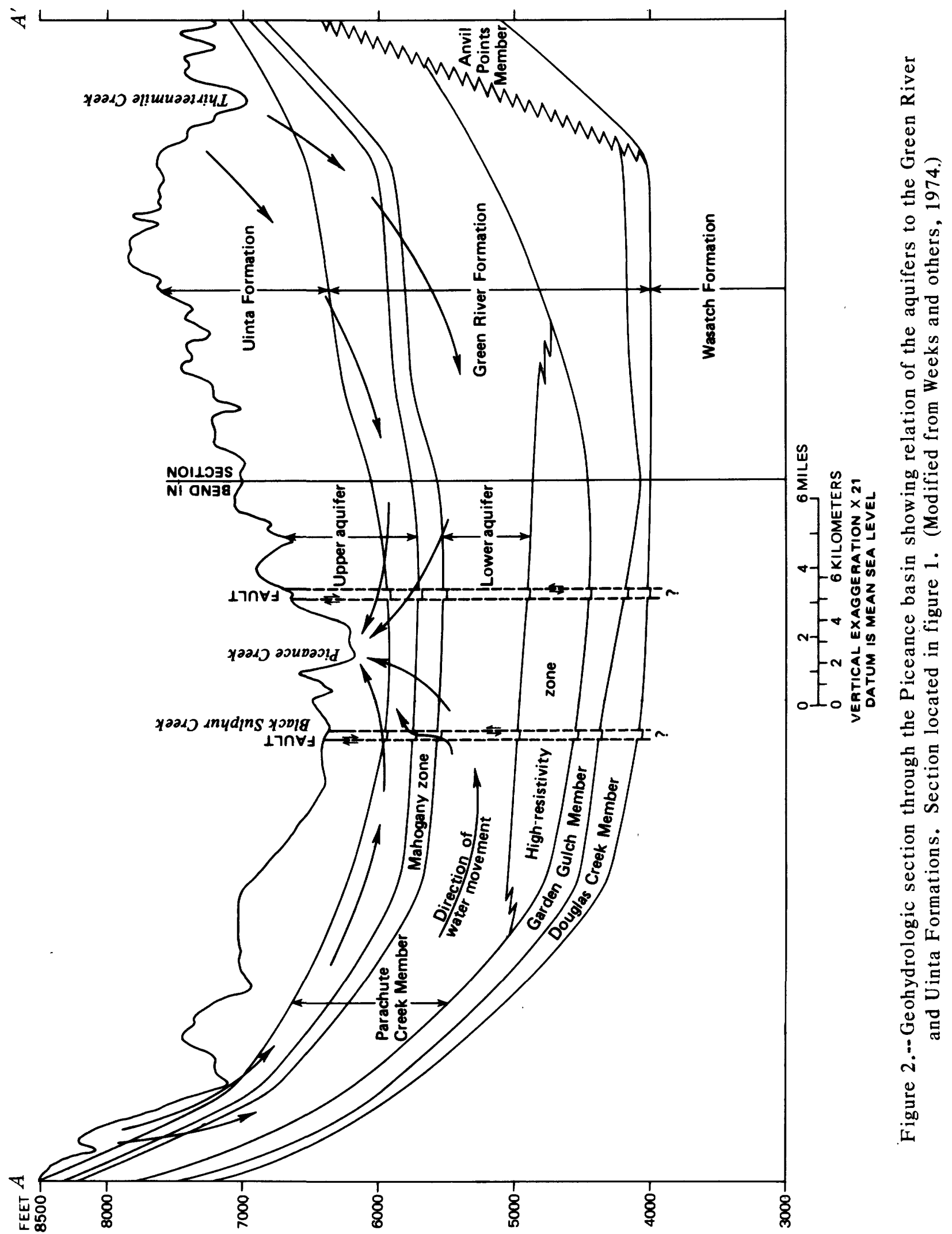


of Eocene age (fig. 2). The permeability of the aquifer is mainly due to secondary or fracture porosity. The upper aquifer is about 1,000 feet thick in the center of the basin and consists of a series of marlstone and sandstone beds with varying permeabilities and degrees of confinement. The aquifer is generally confined by low-permeability sandstones but may be unconfined in many locations, particularly near outcrop areas.

The upper and lower aquifers are separated by the Mahogany zone, an interval of rich oil shale 100 to 200 feet thick. The Mahogany zone extends to all margins of the basin and impedes the flow of water between the aquifers.

The lower aquifer consists of fractured marlstone in the lower part of the Parachute Creek Member below the Mahogany zone (fig. 2). The secondary porosity and permeability of the lower aquifer have been enhanced by the solution of minerals. The thickness of the lower aquifer is generally 400 to 700 feet but may be as much as 1,000 feet. In the central part of the basin, the high-resistivity zone (fig. 2), an interval of rich oil shale, forms the base of the lower aquifer. Where the high-resistivity zone is not present, the Garden Gulch Member of the Green River Formation (fig. 2), which has low permeability, is the base of the aquifer system.

Recharge to the aquifers mainly occurs from snowmelt above an altitude of 7,000 feet along the basin margins. Recharge infiltrates to the upper aquifer and flows toward the north-central part of the basin as illustrated on figure 2. In the recharge area, the hydraulic head in the upper aquifer is higher than that in the lower aquifer and water moves down, through the Mahogany zone, to the lower aquifer. In the north-central part of the basin and in the major stream valleys, the hydraulic heads in the aquifers are reversed and water moves upward from the lower aquifer through the Mahogany zone. Water from the aquifers is eventually discharged in the stream valleys as evapotranspiration and base flow. Ground-water discharge to the white River (fig. 1) is prevented by the structure of the basin.

Very little development of the ground-water resource has occurred in the Piceance basin. Consequently, the ground-water system has not been significantly stressed and changes in the system are due to natural variations in recharge only. Thus, for the purposes of modeling, the aquifer system was assumed to be in a condition of dynamic equilibrium or steady state.

\section{DIGITAL MODEL}

A digital model of ground-water flow in the Piceance basin was developed so that hypothetical dewatering operations for oil-shale mines at tracts $\mathrm{C}-\mathrm{a}$ and $c-b$ (fig. 1) could be simulated. To develop the model, the aquifer system was idealized to permit a mathematical description of the system. 
The upper and lower aquifers are assumed to be horizontal and isotropic. The Mahogany zone confining layer is assumed to permit vertical connection between the aquifers without storage in the confining layer. The flow model assumed for the Piceance basin aquifer system is illustrated on figure 3 by a generalized east-west cross section through the model system. It is assumed that water enters the model aquifer system by recharge from precipitation in the recharge areas at a specified rate. Ground water circulates through the upper and lower aquifers in response to differences in potentiometric heads. The ground water is finally discharged to the stream valley as base flow and evapotranspiration. The lateral and lower boundaries of the aquifer model are assumed to be impermeable so that no water can enter or leave the system by crossing the boundaries. Thus, under steady-state conditions, the rate of recharge must equal the rate of ground-water discharge to the stream valleys.

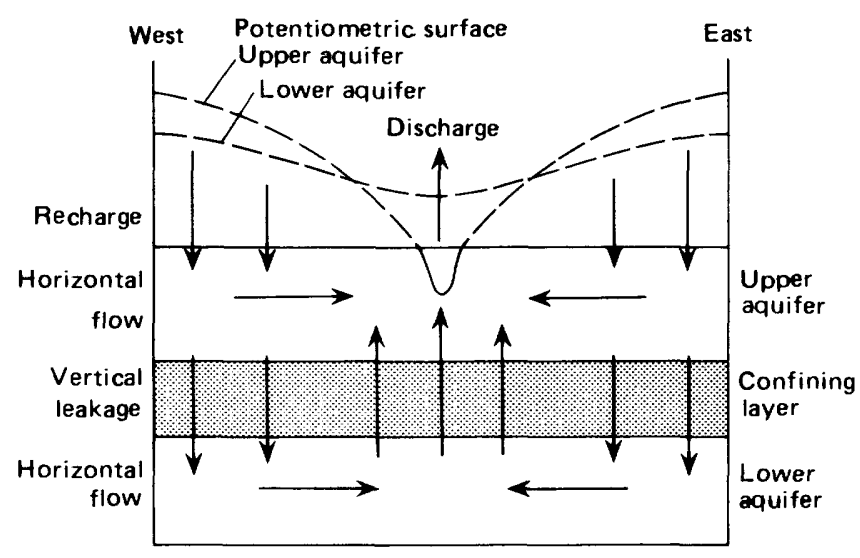

Figure 3.-- Flow model of the aquifer system.

(From Weeks and others, 1974.)

The lateral boundaries of the aquifer model are shown on figure 4 . The lateral boundaries of the model are assumed to be impermeable and coincide with the outcrop of the Green River Formation on the north, east, and west. To the south, the model boundary is assumed to be impermeable and coincide with the ground-water divide on the Roan Plateau. The modeled area is about 900 square miles. The stream valleys of Piceance Creek, Yellow Creek, Dry Fork Piceance Creek, and Black Sulphur Creek (fig. 4) are assumed to be constant-head boundaries in the upper aquifer, and represent areas where ground water is discharged to the stream valleys from the upper aquifer. 


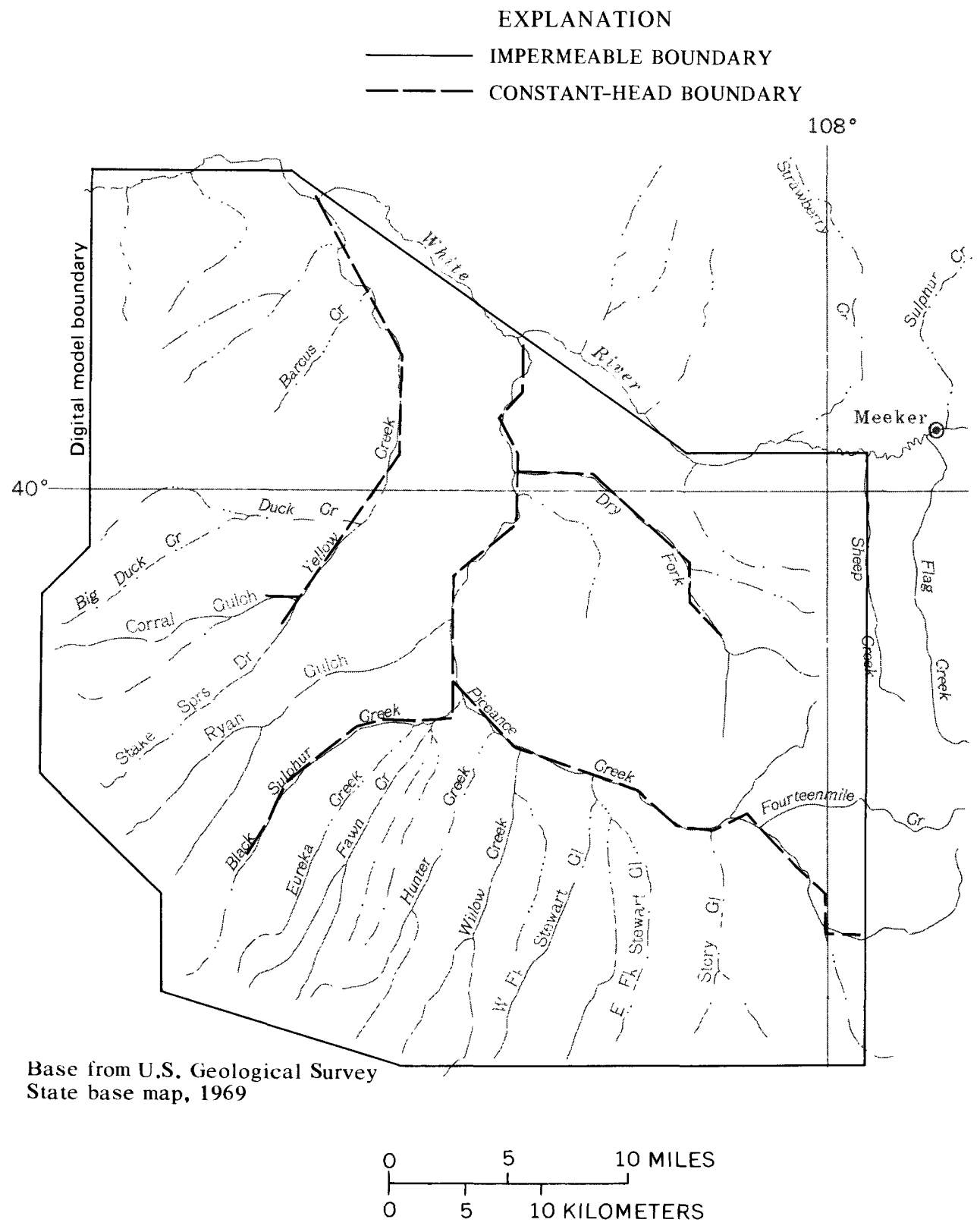

Figure 4.--Aquifer boundaries used in the digital model of the ground-water system. (From Weeks and others, 1974.) 


\section{Mathematical Model}

The digital model used to simulate the multiaquifer system in the Piceance basin was originally developed by Bredehoeft and Pinder (1970). The model is quasi three-dimensional in that it simulates a three-dimensional, multiaquifer system by assuming horizontal flow in the aquifers and vertical flow through the confining layers, or beds, which separate the aquifers. These assumptions reduce the mathematical problem to one of solving coupled two-dimensional equations for each aquifer in the system. An iterative, alternating-direction-implicit scheme is used to solve the system of simultaneous, finite-difference equations which describe the response of the aquifer system to applied stresses.

Bredehoeft and Pinder (1970) tested the model by simulating pumpage from a leaky aquifer system for which the theoretical solution for drawdown was known. The agreement between the numerical solution and the theoretical solution (Bredehoeft and Pinder, 1970, fig. 2) was excellent.

\section{Ground-Water Flow Equation}

The general equation which governs the flow of water in a two-dimensional, isotropic, confined aquifer is:

$$
\frac{\partial}{\partial x}\left(T \frac{\partial h}{\partial x}\right)+\frac{\partial}{\partial y}\left(T \frac{\partial h}{\partial y}\right)=S \frac{\partial h}{\partial t}+W(x, y, t)
$$

where $T$ is the transmissivity of the aquifer $\left(L^{2} / t\right)$,

$h$ is the hydraulic head in the aquifer (L),

$S$ is the storage coefficient of the aquifer (dimensionless), and

$W(x, y, t)$ is the flow rate per unit area of a source or sink $(L / t)$.

The transmissivity and storage coefficient are both functions of the space variables $x$ and $y$. The source term is a function of the space variables, time, $t$, and also may be a function of hydraulic head. The source term, $W$, incorporates the effects of natural recharge or discharge, recharge or discharge from wells, and leakage from adjacent aquifers. For leakage without storage in the confining bed, the vertical flow through the confining bed to an adjacent aquifer is given by:

$$
L=\frac{K^{\prime}}{m}(\hat{h}-h),
$$

where $L$ is the flow rate per unit area $(L / t)$,

$\hat{h}$ is the hydraulic head in the adjacent aquifer (L),

and

$K^{\prime}$ is the vertical hydraulic conductivity of the confining layer $(\mathrm{L} / \mathrm{t})$,

$m$ is the thickness of the confining layer (L).

Equation 1 can be written for both the upper and lower aquifers. Substitution of $L$, along with other appropriate source and (or) sink terms, for $W$ 
couples the equations describing the head distribution in the two adjacent aquifers.

The appropriate boundary and initial conditions required to obtain a unique solution to equation 1 depend on the conceptual model. The boundaries of the conceptual model are assumed to be impermeable. Therefore, the boundary conditions applicable to equation 1 require that the partial derivative of the head, $h$, in the direction normal to the aquifer boundary must be zero. For transient-flow problems, the initial conditions require specifying the head, $h$, as a function of the space variables, $x$ and $y$, at time zero. For steady-state flow, the solution to equation 1 is independent of time and, therefore, independent of initial conditions.

\section{Finite-Difference Approximations}

To solve equation 1 for heterogeneous aquifers with irregular boundaries, the aquifers are subdivided into rectangular blocks in which the aquifer properties are assumed to be uniform. The derivatives in equation 1 are replaced by finite-difference approximations for the derivatives at the center of each block. The result is a system of simultaneous algebraic equations (Bredehoeft and Pinder, 1970) which can be solved efficiently by digital computers.

The finite-difference approximations to equation 1 were developed for a block-centered grid with variable grid spacing. The index scheme used for the grid system is shown on figure 5. The approximation method used is known as the iterative alternating-direction implicit procedure (Douglas and Rachford, 1956). The procedure consists of two steps involving the solution of two tridiagonal sets of equations for each aquifer. The first tridiagonal set of equations is derived by expressing one space derivative implicitly and the other explicitly in terms of known head values from the previous iteration. The next step is to reverse the procedure of the first step utilizing the intermediate solution as known values. Thus, the procedure is to alternate the direction of the implicit and explicit derivatives. For the calculation by rows $\left(\partial^{2} h / \partial x^{2}\right.$ implicit), equation 1 can be approximated by

$$
\begin{aligned}
\frac{1}{\Delta x_{i}}\left[\left(T \frac{\partial h}{\partial x}\right)_{i+\frac{1}{2}, j, k}^{n+\frac{1}{2}}\right. & \left.-\left(T \frac{\partial h}{\partial x}\right)_{i-\frac{1}{2}, j, k}^{n+\frac{1}{2}}\right]+\frac{1}{\Delta y_{j}}\left[\left(T \frac{\partial h}{\partial y}\right)_{i, j+\frac{1}{2}, k}^{n}-\left(T \frac{\partial h}{\partial y}\right)_{i, j-\frac{1}{2}, k}^{n}\right] \\
& =\frac{S_{i, j}}{\Delta t_{k}}\left(h_{i, j, k}^{n+\frac{1}{2}}-h_{i, j, k-1}\right)+w_{i, j, k}+I_{i}\left(h_{i, j, k}^{n+\frac{1}{2}}-h_{i, j, k}^{n}\right),
\end{aligned}
$$

where $\Delta x_{i}$ is the width of column $i$ (fig. 5) (L), $\Delta y_{j}$ is the height of row $j$ (fig. 5) (L), $\Delta t_{k}$ is the $k^{\text {th }}$ time increment $(t)$, 


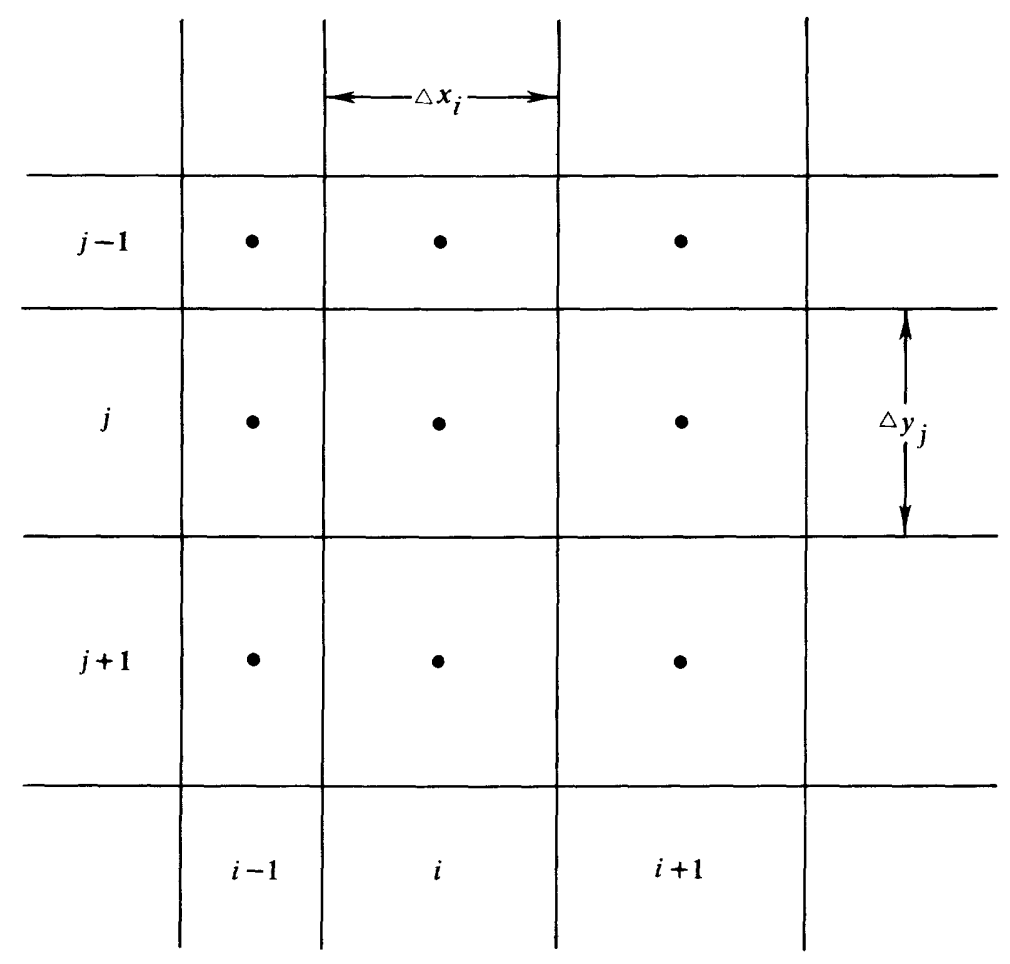

Figure 5..- Index scheme for finite-difference grid at block $(i, j)$.

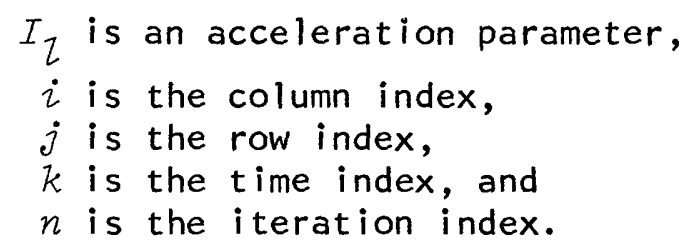

The purpose of the acceleration parameter is to reduce the number of iterations to converge to a solution.

The derivatives in equation 3 can be approximated as follows:

$$
\left(T \frac{\partial h}{\partial x}\right)_{i+\frac{1}{2}, j, k}^{n+\frac{1}{2}}=\left(\frac{T}{\Delta x}\right)_{i+\frac{1}{2}, j}\left(h_{i+1, j, k}^{n+\frac{1}{2}}-h_{i, j, k}^{n+\frac{1}{2}}\right) .
$$

The ratio $(T / \Delta x)_{i+\frac{1}{2}, j}$ in equation 4 must be approximated using known values at blocks $(i, j)$ and $(i+1, j)$. The ratio is usually approximated by the harmonic mean of the ratios at the two neighboring nodes. Thus, if

$$
\left(\frac{T}{\Delta x}\right)_{i+\frac{1}{2}, j}=\frac{2 T i+1, j^{T} i, j}{\Delta x_{i}^{T} i+1, j+\Delta x_{i+1}^{T} i, j},
$$


and

$$
\begin{aligned}
& A_{i, j}=\frac{1}{\Delta x_{i}}\left(\frac{T}{\Delta x}\right)_{i-\frac{1}{2}, j}, \\
& C_{i, j}=\frac{1}{\Delta x_{i}}\left(\frac{T}{\Delta x}\right)_{i+\frac{1}{2}, j}, \\
& E_{i, j}=\frac{1}{\Delta y_{j}}\left(\frac{T}{\Delta y}\right)_{i, j-\frac{1}{2}}, \\
& F_{i, j}=\frac{1}{\Delta y_{j}}\left(\frac{T}{\Delta y}\right)_{i, j+\frac{7}{2}},
\end{aligned}
$$

equation 3 for row computations can be written as

$$
\begin{aligned}
A_{i, j}\left(h_{i-1, j, k}^{n+\frac{1}{2}}\right. & \left.-h_{i, j, k}^{n+\frac{1}{2}}\right)+C_{i, j}\left(h_{i+1, j, k}^{n+\frac{1}{2}}-h_{i, j, k}^{n+\frac{1}{2}}\right) \\
& +E_{i, j}\left(h_{i, j-1, k}^{n}-h_{i, j, k}^{n}\right)+E_{i, j}\left(h_{i, j+1, k}^{n}-h_{i, j, k}^{n}\right) \\
& =\frac{S_{i, j}}{\Delta t_{k}}\left(h_{i, j, k}^{n+\frac{1}{2}}-h_{i, j, k-1}\right)+w_{i, j, k}+I_{i}\left(h_{i, j, k}^{n+\frac{I}{2}}-h_{i, j, k}^{n}\right) .
\end{aligned}
$$

Similarly, the equation for column computations ( $\partial^{2} h / \partial^{2} y$ implicit) is

$$
\begin{aligned}
A_{i, j}\left(h_{i-1, j, k}^{n+\frac{1}{2}}\right. & \left.-h_{i, j, k}^{n+\frac{1}{2}}\right)+C_{i, j}\left(\begin{array}{c}
h_{i+1, j, k}^{n+\frac{1}{2}} \\
n_{i, j, k}
\end{array}\right) \\
& +E_{i, j}^{n+\frac{1}{2}}\left(h_{i, j-1, k}^{n+1}-h_{i, j, k}^{n+1}\right)+E_{i, j}\left(h_{i, j+1, k}^{n+1}-h_{i, j, k}^{n+1}\right) \\
& =\frac{S_{i, j}}{\Delta t_{k}}\left(h_{i, j, k}^{n+1}-h_{i, j, k-1}\right)+w_{i, j, k}+I_{i}\left(h_{i, j, k}^{n+1}-h_{i, j, k}^{n+\frac{1}{2}}\right) .
\end{aligned}
$$

An iteration consists of alternately solving equations 7 and 8 for each aquifer in the system. For the model of the Piceance basin, there are two aquifers and an iteration consists of solving the row and column equations for the upper aquifer and repeating the procedure for the lower aquifer. For each time step, iteration continues until the greatest head difference between consecutive iterations is less than a prescribed error or tolerance. When closure is achieved, the procedure is repeated for the next time step. 
The acceleration parameters, $I_{Z}$, are calculated by the model from the equation

$$
I_{z}=\omega_{z}\left(A_{i, j}+C_{i, j}+E_{i, j}+E_{i, j}\right) \text {, }
$$

where the iteration parameter, $\omega_{l}$, ranges between a maximum value of one and a minimum value computed from

$$
\begin{aligned}
\omega_{\min }=\operatorname{Min}(\text { for all } i, j) & {\left[\frac{\pi^{2}}{2 N_{x}^{2}} \frac{1}{1+\frac{\Delta x_{i} C_{i, j}\left(\Delta y_{j}\right)^{2}}{\Delta y_{j} F_{i, j}\left(\Delta x_{i}\right)^{2}}},\right.} \\
& \left.\frac{\pi^{2}}{2 N_{y}^{2}} \frac{1}{\left.1+\frac{\Delta y_{j}{ }^{F} i, j}{\Delta x_{i} C_{i, j}\left(\Delta x_{i}\right)^{2}}\right)^{2}}\right],
\end{aligned}
$$

where $N_{x}$ is the number of columns and $N_{y}$ is the number of rows. Recall from equation 6 that $\Delta x_{i} C_{i, j}$ and $\Delta y_{i} F_{i, j}$ are the harmonic means of the ratios as defined by equation 5 . The set of parameters, $\omega_{l}$, are spaced in a geometric sequence by the formula

$$
\omega_{z+1}=\omega_{z} \exp \left[\frac{\ln \left(1 / \omega_{\min }\right)}{M-1}\right],
$$

where $M$ is the number of iteration parameters selected by the user. The parameters, $\omega_{l}$, are computed once in the model and stored in an array. As iteration proceeds, beginning with $\omega_{1}=\omega_{m i n}$, the iteration parameters are cycled. The acceleration parameter, $I_{Z}$, is calculated from equation 9 during the solution of each set of row or column equations.

The optimum number of iteration parameters is problem dependent. For the Piceance basin model, five to seven iteration parameters will provide rapid convergence for transient solutions. For a more detailed discussion of the iteration parameters, see Trescott, Pinder, and Larson (1976, p. 20).

The source term, $W_{i, j, k}$, incorporates the effects of pumpage, recharge, and leakage through the confining layers. Assuming outflow is positive and inflow is negative,

$$
W_{i, j, k}=\frac{Q_{i, j}}{\Delta x_{i} \Delta y_{j}}-R_{i, j}+L_{i, j, k},
$$


where $Q_{i, j}$ is the well discharge rate at block $(i, j)\left(L^{3} / t\right)$,

$R_{i, j}$ is the recharge rate per unit area of block $(i, j)(L / t)$, and

$L_{i, j, k}$ is the leakage (flow rate per unit area) to adjacent aquifers at block $(i, j)$ during the $k^{\text {th }}$ time step $(L / t)$.

The well discharge rate $\left(Q_{i, j}\right)$ and the recharge velocity (rate per unit area, $R_{i, j}$ ) are specified at each block in the model. Well discharge is assumed to be constant for a specified pumping period which may include several time steps. The recharge rate is assumed to be constant throughout the period of simulation. However, leakage through the confining layers (including discharge to constant-head boundaries) is computed by the model during each iteration. For the upper aquifer,

$$
L_{i, j, k}=V_{i, j}\left(h_{i, j, k}^{r}-W T i, j\right)+\hat{V}_{i, j}\left(\hbar_{i, j, k}^{r}-\hat{h}_{i, j, k}^{n}\right) \text {, }
$$

where ${ }^{W T}{ }_{i, j}$ is the constant hydraulic head in the upper aquifer at $(i, j)(L)$,

$V_{i, j}$ is the leakance $\left(K^{\prime} / m\right)$ of the layer confining the upper aquifer

$\hat{h}_{i, j, k}^{n}$ denotes the hydraulic head in the lower aquifer at $(i, j, k)$

calculated during the previous $\left(n^{\text {th }}\right)$ iteration $(L)$, $\hat{V}_{i, j}$ is the leakance of the confining layer separating the upper and

$r$ is the iteration index which equals $n+\frac{3}{2}$ for row computations and $n+1$ for column computations.

The constant hydraulic heads $(W T, j, j$ ) and the leakance of the upper confining layer $\left(V_{i, j}\right)$ are convenience arrays used to control discharge from (or recharge to) the upper aquifer to surface streams (constant-head boundaries). For example, if a constant head at block $(i, j)$ in the upper aquifer is desired, then the desired constant head is assigned to $W T i, j$ and an extremely large leakance value is assigned to $V_{i, j}$. Application of Darcy's law causes the hydraulic head in the upper aquifer, $h_{i, j, k}$, to be about equal to $W T i, j$. The values initially assigned to $W T T_{i, j}$ are never changed during computing.

Although not applicable to the model of the Piceance basin, the WT and $V$ arrays can be used to simulate flux between the upper aquifer and an overlying water-table aquifer. In addition, the arrays can be used to model the effects of low permeability stream sediments on ground-water discharge to streams or other surface-water bodies. 
by

For the lower aquifer, the leakage through the confining layer is given

$$
L_{i, j, k}=\hat{V}_{i, j}\left(\hat{h}_{i, j, k}^{r}-h_{i, j, k}^{n}\right)
$$

Equation 7 can now be rearranged so that all unknown quantities are on the left side of the equation and all known quantities are on the right. By substituting equations 12 and 13 for $w_{i, j, k}$ in equation 7 and rearranging, the equation for row computations in the upper aquifer becomes

$$
\begin{aligned}
A_{i, j} h_{i-1, j, k}^{n+\frac{1}{2}}- & {\left[(A+C+V+\hat{V})_{i, j}+\frac{S_{i, j}}{\Delta t_{k}}+I_{l}\right]_{i, j, k}^{n+\frac{1}{2}}+C_{i, j} h_{i+1, j, k}^{n+\frac{1}{2}} } \\
= & -E_{i, j} h_{i, j-1, k}^{n}+\left(E_{i, j}+F_{i, j}-I_{l}\right) h_{i, j, k}^{n}-F_{i, j} h_{i, j+1, k}^{n} \\
& -\frac{S_{i, j}}{\Delta t_{k}}{ }_{i, j, k-1}-V_{i, j} W T_{i, j}-\hat{V}_{i, j} \hat{h}_{i, j, k}^{n}+\frac{Q_{i, j}}{\Delta x_{i} \Delta y_{j}}-R_{i, j},
\end{aligned}
$$

which can be simplified to

$$
A_{i, j} h_{i-1, j, k}^{n+\frac{1}{2}}+B_{i, j} h_{i, j, k}^{n+\frac{1}{2}}+C_{i, j} h_{i+1, j, k}^{n+\frac{1}{2}}=D_{i, j},
$$

where $B_{i, j}$ and $D_{i, j}$ are defined by equation 15 . This system of equations is readily solved by the computer program using Gaussian elimination (Carnahan and others, 1969, p. 441).

The form of equation 16 is identical for row or column computation in both the upper and lower aquifers. For column computations in the upper aquifer,

$$
a_{i, j} h_{i, j-1, k}^{n+1}+b_{i, j} n_{i, j, k}^{n+1}+c_{i, j} h_{i, j+1, k}^{n+1}=a_{i, j},
$$

where $a_{i, j}=E_{i, j}$,

$$
\begin{aligned}
& b_{i, j}=-(\alpha+c+V+\hat{V})_{i, j}-\frac{s_{i, j}}{\Delta t_{k}}-I_{\eta}, \\
& c_{i, j}=F_{i, j},
\end{aligned}
$$

and

$$
\begin{aligned}
d_{i, j}= & -A_{i, j} h_{i-1, j, k}^{n+\frac{1}{2}}+\left(A_{i, j}+C_{i, j}-I_{\imath}\right)_{i, j, k}^{n+\frac{1}{2}}-C_{i, j} h_{i+1, j, k}^{n+\frac{1}{2}} \\
& -\frac{S_{i, j}}{\Delta t_{k}} h_{i, j, k-1}-V_{i, j} W T_{i, j}-\hat{V}_{i, j} \hat{h}_{i, j, k}^{n}+\frac{Q_{i, j}}{\Delta x_{i} \Delta y_{j}}-R_{i, j} .
\end{aligned}
$$


Equations 16 and 17 also apply to row and column computations in the lower aquifer if $h$ is replaced by $\hat{h}$ and the coefficients are computed from transmissivity and storage-coefficient data for the lower aquifer. The coefficients which are different are those involving the leakage term (see equations 13 and 14 ).

Before equations 16 and 17 can be solved, values must be assigned to all parameters from which the coefficients are computed. The values assigned to the parameters become input data to the computer program. The input data that must be provided are the block dimensions, transmissivity and storage coefficients of the aquifers, leakance of the confining layers, initial hydraulic heads in the aquifers, and the recharge velocity and pumping rate for each node in the model. The input data are included in a listing at the back of this report and will be discussed later. The development of the data is discussed by Weeks, Leavesley, Welder, and Saulnier (1974).

\section{Model Geometry}

The digital model is based on a rectangular block-centered grid which permits irregular grid spacing. The planar area of the model is divided into a rectangular grid with the origin of the coordinate system at the northwest corner. The map reference lines for the grid are shown on figure 6 and the grid spacing is given in the input data listing for the computer program. The grid system used for the Piceance basin aquifer system consists of 29 columns and 33 rows. The grid spacing ranges from 1.0 to 2.8 miles. The locations of constant-head nodes, which correspond to the location of streams hydraulically connected to the upper aquifer, are shown on figure 6 also. The grid is constructed so that at least one block at the beginning and end of each row and column is outside the modeled area. This permits irregular model boundaries within the rectangular grid. The finite-difference grid is used to overlay maps of aquifer boundaries, transmissivity, storage coefficient, leakance, and recharge so that values of the parameters required for computational purposes can readily be coded on card format for input to the model.

\section{COMPUTER PROGRAM}

The computer program is written in FORTRAN IV utilizing variables which will enable users to adapt the model to other multiaquifer systems.

\section{Program Variables}

The principal variables used in the computer program are defined in table 1. Several other variables are used in the program for intermediate computations or array indices. The use of these variables is generally selfexplanatory in the program. 


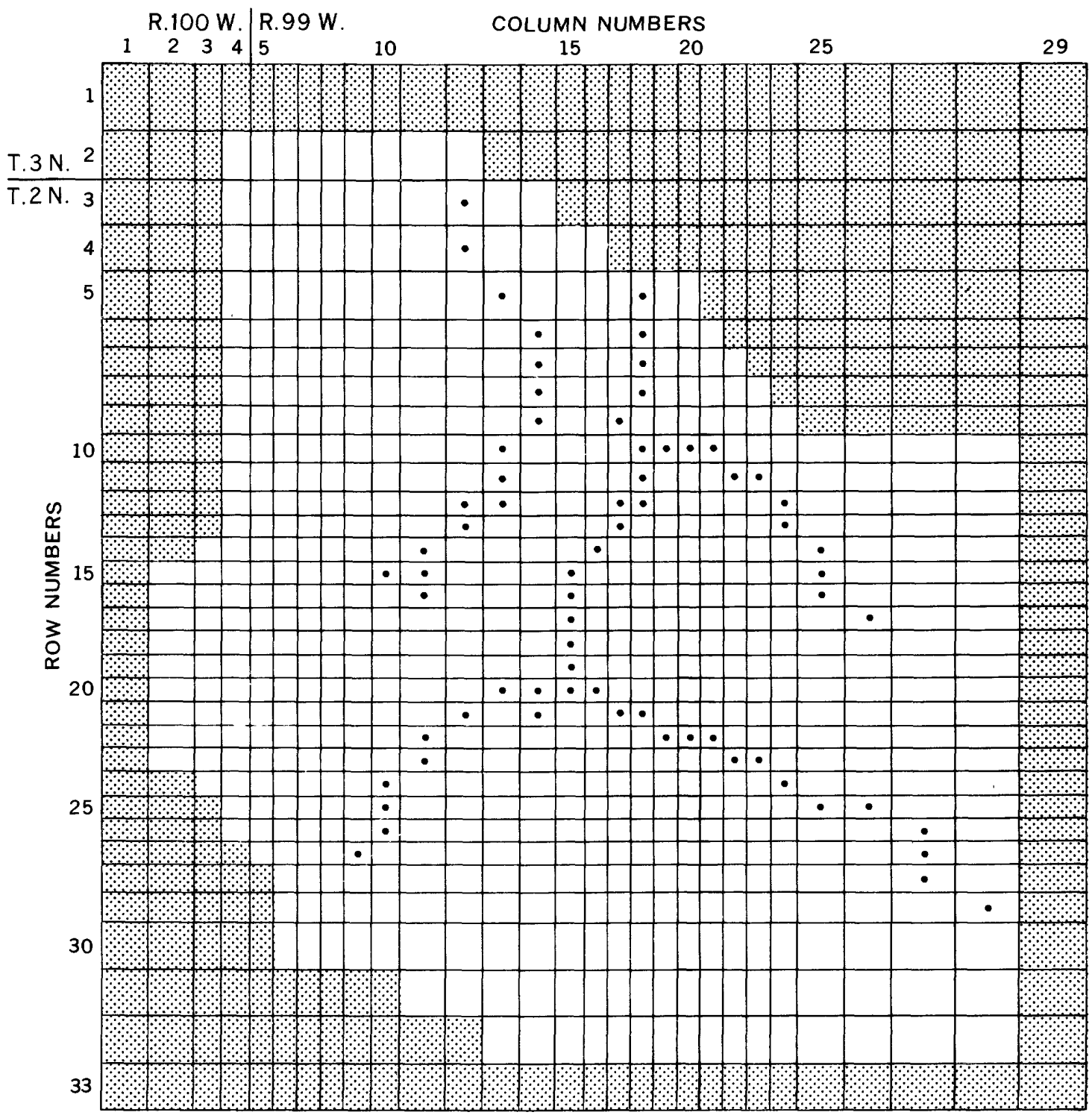

EXPLANATION

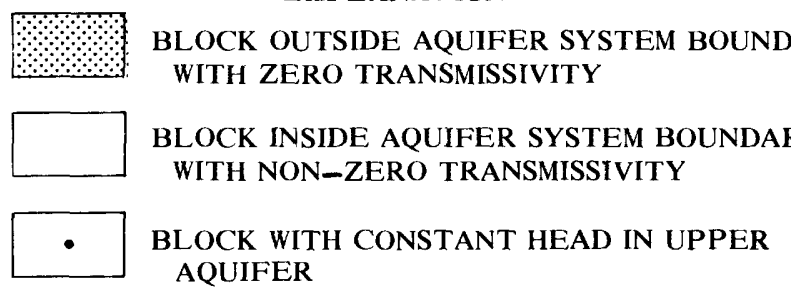

Figure 6..-Block-centered, finite-difference grid used to model the Piceance basin aquifer system showing township and range reference lines and grid-numbering system. 
Table 1.--Definitions of variables used in the computer program which simulates ground-water flow in the Piceance basin

Variable

AOPT (NITP)

$B A L$

FCTR

FLUXI

FLUX2

$H C(N X, N Y, N L)$

$H I(N X, N Y, N L)$

$H K(N X, N Y, N L)$

HMIN

$H R(N X, N Y, N L)$

ID

$\mathrm{IH}(\mathrm{NX})$

IL

INT

I PMP

IRHI

KOUNT

$\mathrm{N}$

NITP

NL

NPMP

NPNT

NREC

NTIM

$N X$

NY

OPTP

PARAM

PINT (NPMP)

PUMP

PYR

$Q(N X, N Y)$

QIN

QOUT

QSTR

$\mathrm{R} 1, \mathrm{R} 2, \mathrm{R} 3$

$R C I(N X, N Y, N L)$

$\operatorname{REC}(N X, N Y, N L)$

RECH

REMN
Definition

Array of iteration parameters.

Transient mass balance.

Scale factor for input data.

Net outflow at start of time period.

Net outflow at end of time period.

Array of head values, column solution.

Array of initial head values.

Array of head values, time step solution.

Minimum iteration parameter.

Array of head values, row solution.

Directional index for transmissivity array.

Array of head differences.

Aquifer or confining layer index.

Pumping period index.

Mass balance option variable.

Initial head option variable.

Iteration counter.

Time step index.

Number of iteration parameters.

Number of aquifers or confining layers.

Number of pumping periods.

Print control option variable.

Number of pumping nodes.

Number of time steps.

Number of columns.

Number of rows.

Output option variable.

I teration parameter.

Array of pumping periods.

Total pumping rate.

Summation of pumping periods.

Outflow rate at constant head nodes.

Summation of inflow rate.

Summation of outflow rate.

Volume of water from aquifer storage.

Recharge rate factors.

Array of uniform recharge rates.

Array of recharge + pumping rates.

Total recharge rate.

Print control variable. 
Table 1.--Definitions of variables used in the computer program which simulates ground-water flow in the Piceance basin--Continued

\begin{tabular}{ll}
\multicolumn{1}{c}{ Variable } & \multicolumn{1}{c}{ Definition } \\
\hline RCE & Pumping rate at node. \\
S $(N X, N Y, N L)$ & Storage coefficient array. \\
SUMT & Summation of time. \\
TEST & Convergence test variable. \\
TDEL & Time step. \\
THCK $(N X, N Y, N L)$ & Aquifer thickness array. \\
TIM $(N T I M)$ & Time step array. \\
TMRX $(N X, N Y, I D)$ & Harmonic-mean transmissivity array. \\
TOL & Convergence tolerance. \\
VFLUX & Volume of flow to constant-head nodes. \\
VPRM $(N X, N Y, N L)$ & Leakance or transmissivity array. \\
VPUMP & Volume of pumping. \\
VRECH & Volume of recharge. \\
WT $(N X, N Y)$ & Array of constant hydraulic heads. \\
XDEL $(N X)$ & X-spacing array. \\
YDEL $(N Y)$ & Y-spacing array.
\end{tabular}

\section{Program Options}

The computer program has several options available to the user. These options control output printing, computations, and data input. Options are assigned values in SUBROUTINE PARLOD between line numbers B46 and B51. The line numbers appear at the right end of each line in the program listing. The option variables have assigned values as follows:

Line B46: NPNT is a print control index. For transient-flow problems, the head solution matrix is printed out every NPNT time steps. For steadystate problems, the head solution is achieved during the first time step and NPNT should be assigned an integer value greater than one.

Line B47: OPTP controls the printing of the hydraulic head solution matrix. If OPTP is assigned the value 1 , the solution matrix is printed in tabular form. If OPTP is assigned the value 2 , the solution matrix is printed in tabular and map form. The hydraulic-head maps can be contoured by hand although the map is not to scale. If OPTP is assigned the value 3 , the solution matrix is printed in tabular and map form and punched on card format. The punched output is for use as initial conditions for subsequent simulations or as input to a separate computer program to contour the solution at a suitable map scale. 
Line B48: IPMP controls the mass-balance computations. If IPMP is set to zero, no mass balance will be computed. A mass balance is calculated if IPMP is assigned the value 1 for steady-state solutions or 2 for transient solutions.

Line B49: IRHI controls the method of inputing initial hydraulic-head values. If IRHI is equal to 1 , initial hydraulic heads are read in card format. If $\mid R H I$ is equal to 2 , initial hydraulic heads are assigned in SUBROUTINE BOUDY.

Line B50: NITP is the number of iteration parameters to be computed in SUBROUTINE PARLOD. The purpose of the iteration parameters is to speed convergence to a solution. The optimum number of parameters is problem dependent. For the two-aquifer model of the Piceance basin, five to seven iteration parameters were found to provide rapid convergence for transient problems. Simulation of steady-state conditions in the Piceance basin resulted in extremely slow convergence irrespective of the number of iteration parameters when relatively large leakance values were used. Rapid convergence to a steady-state solution was achieved by obtaining a solution for the upper aquifer $[N L=1$ and $\operatorname{VPRM}(N X, N Y, 2)=0]$. The solution for the upper aquifer was then used as initial conditions for the upper aquifer and lower aquifer in the two-aquifer model. Rapid convergence was then obtained.

Line B51: TOL is the parameter used to test for convergence to a solution. Convergence is achieved when the hydraulic head at every node in each aquifer does not change in absolute value by an amount greater than TOL, in feet, between successive iterations. The magnitude of TOL should depend on the adequacy of the data to meet input-data requirements (a subjective evaluation by the user) and the range in hydraulic-head values expected in the solution. However, the value of TOL must be small enough to obtain a satisfactory solution as indicated by the mass balance. In the model of the Piceance basin aquifer system, values of 0.1 foot for steady-state solutions and 1.0 foot for transient solutions were assigned to TOL.

\section{Input Variables}

Input data required by the computer program are either read from cards or assigned within the program. The formats for data read by the program are contained in the program listing and summarized in table 2 .

The following variables are read or assigned values at the line numbers indicated in SUBROUTINE PARLOD.

Line B20: NTIM is the number of time steps per pumping period to be assigned to the array TIM. For a steady-state solution, NTIM is assigned the value 1. For transient solutions, NTIM must be assigned a value such that the summation of NTIM time steps (SUMT) is equal to or greater than the longest pumping period. The value of NTIM must be less than 100 (the dimension of array $T(M)$. The last time increment (TDEL) in each pumping period is adjusted 
Table 2.--FORMATs for input variables read by the computer program

\begin{tabular}{|c|c|c|c|}
\hline $\begin{array}{l}\text { Order } \\
\text { read }\end{array}$ & $\begin{array}{l}\text { Variable and } \\
\text { dimension }\end{array}$ & $\begin{array}{l}\text { READ } \\
\text { FORMAT }\end{array}$ & $\begin{array}{c}\text { FORMAT line } \\
\text { number }\end{array}$ \\
\hline $\begin{array}{l}1-\cdots-- \\
2-\cdots-- \\
3-\cdots-- \\
4-\cdots-- \\
5-\cdots--\end{array}$ & $\begin{array}{l}\operatorname{PINT}(25) \\
\operatorname{XDEL}(29) \\
\operatorname{YDEL}(33) \\
\operatorname{VPRM}(29,33,2) \\
\operatorname{VPRM}(29,33,2)\end{array}$ & $\begin{array}{l}25 F 3.0 \\
29 F 2.0 \\
33 F 2.0 \\
29 F 2.0 \\
29 F 2.0\end{array}$ & $\begin{array}{ll}B & 114 \\
C & 148 \\
C & 149 \\
C & 146 \\
C & 146\end{array}$ \\
\hline $\begin{array}{r}6-\cdots-- \\
7--\cdots \\
8-\cdots-- \\
9-\cdots-- \\
10-\cdots-\end{array}$ & $\begin{array}{l}\text { WT }(29,33) \\
\text { HI }(29,33,2) \\
\operatorname{RCI}(29,33,2) \\
\text { NREC } \\
\text { IX, IY, IL, RCE }\end{array}$ & $\begin{array}{c}\text { 10F8.2/10F8.2/9F8.2 } \\
10 F 8.2 \\
29 F 2.0 \\
113 \\
313, F 10.5\end{array}$ & $\begin{array}{ll}\text { D } & 65 \\
\text { D } & 67 \\
\text { E } & 78 \\
\text { E } & 75 \\
\text { E } & 74\end{array}$ \\
\hline
\end{tabular}

in SUBROUTINE ITERAT so that the solution will be obtained at exactly the end of each pumping period.

Line B21: NPMP is the number of pumping periods to be simulated. NPMP must be assigned the value 1 for steady-state solutions and as much as 25 (dimension of PINT) for transient solutions.

Line B22: $N X$ is the number of columns in the solution matrix or the number of blocks in the $X$-direction of the finite-difference grid. The value of NX is 29 in the model of the Piceance basin.

Line B23: $N Y$ is the number of rows in the solution matrix or the number of blocks in the Y-direction of the finite-difference grid. The value of NY is 33 in the model of the Piceance basin.

Line B24: NL is the number of aquifers or confining layers simulated. The value of $\mathrm{NL}$ is 2 in the model of the Piceance basin. The upper aquifer and overlying confining layer is indexed by the number 1 and the lower aquifer and its overlying confining layer is indexed by the number 2 .

Line B67: PINT(NPMP) is an array of pumping intervals. NPMP pumping intervals are read from card format (table 2) in convenient units. The variable FCTR is used to change the units of PINT to seconds. At the beginning of each pumping period, subroutine RCHRG is called and the number of pumping nodes (NREC) to be simulated during the pumping period is read. (See discussion of NREC.)

Line B87: TIM(NTIM) is an array of time increments, in seconds, which are used to simulate the duration of each pumping period. The time-step variable (TDEL) in SUBROUTINE ITERAT is assigned successive values from the 
array TIM until calculations have proceeded the maximum number of time steps (NTIM) or the end of the pumping period is reached. For transient-flow problems, the duration, in seconds, of each time increment (TIM) may be assigned successively larger values. In line B84, the first time step, TIM(1), is assigned a value, in seconds, from which successive time steps are computed. In fact, a pumping period can be simulated by a single time step although the number of iterations required for convergence may become excessive. An initial time step of about $1 / 100$ of the shortest pumping period is frequently used with successive time increments multiplied by a factor of 1.5 or 2.0. For steady-state solutions, any initial time step may be used.

The following variables are read or assigned values at the line numbers indicated in SUBROUTINE TRANS.

Line C19: XDEL(NX) is an array of column widths (finite-difference grid spacing in the X-direction). NX column widths are read from card format (table 2) in convenient units. The variable FCTR is used to change the units of XDEL to feet. In the program for the Piceance basin, XDEL is read in units of $0.2 \mathrm{mile}$.

Line C20: YDEL(NY) is an array of row heights (finite-difference grid spacing in the $Y$-direction). NY row heights are read from card format (table 2) in convenient units. The variable FCTR is used to change the units of YDEL to feet. In the program for the Piceance basin, YDEL is read in units of $0.2 \mathrm{mile}$.

Line C43: $S(N X, N Y, N L)$ is an array of aquifer storage coefficients. The storage coefficients for NL aquifers are assigned values in the program. For steady-state solutions, the array of storage coefficients must be set equal to zero. For transient-flow solutions with the Piceance basin model, uniform storage coefficients of 0.001 were assigned to the upper aquifer $(N L=1)$ and 0.0001 to the lower aquifer $(N L=2)$.

Line C57: VPRM(NX,NY,NL) is an array used to read the transmissivity of NL aquifers and the leakance of NL confining layers. The transmissivity of the aquifers is read from card format (table 2) in convenient units. The variable FCTR is used to change the units of VPRM to feet squared per second. In the model of the Piceance basin, the transmissivity is read in units of 100 gallons per day per foot. The directional harmonic-mean transmissivities (TMRX) are then computed from VPRM, XDEL, and YDEL for each node in the model. Elements of the array THCK are then assigned values of 1.0 at nodes where VPRM is not zero. One or more consecutive elements at the beginning and end of each row and column in the transmissivity matrix must be zero. These elements correspond to nodes in the finite-difference grid which are outside the aquifer boundaries. Zero values of transmissivity at nodes within the aquifer boundaries are not allowed.

Line C120: After the transmissivity data have been read into the program, the leakance of $N L$ confining layers is read from card format (table 2) into the array $\operatorname{VPRM}(N X, N Y, N L)$ in convenient units. The variable FCTR is 
used to change the units of VPRM to inverse seconds. The leakance of the upper confining layer $(\mathrm{NL}=1)$ is used to simulate the stream-aquifer interface. A negative value of leakance is used to flag nodes along the stream courses where ground-water discharge or recharge occurs. An appropriate leakance value is then assigned in the program (line C134) to the corresponding negative element of VPRM $(N X, N Y, 1)$ to simulate hydraulic connection between the stream and aquifer. In the Piceance basin model, an extremely large leakance value $\left(1.0 \mathrm{~s}^{-1}\right)$ is assigned to these elements. In effect, the large leakance value creates a constant head at each node on the stream course. A smaller value could be used to simulate impedance to flow at the stream-aquifer interface. All other elements of the array $\operatorname{VPRM}(N X, N Y, 1)$ are assigned zero values. In the Piceance basin model, VPRM $(N X, N Y, 2)$ is the leakance of the ccnfining layer separating the upper and lower aquifers and a uniform value of $1.55 \times 10^{-10} \mathrm{~s}^{-1}$ is assigned to the array.

The following variables are read or assigned values at the 1 ine numbers indicated in SUBROUTINE BOUDY.

Line D18: $W T(N X, N Y)$ is an array of constant hydraulic heads used to control leakage at the stream-aquifer interface and create constant-head nodes in the upper aquifer $(N L=1)$. Elements of the array WT are read, in feet, from card format. A constant head at node $\mathrm{I}, \mathrm{J}$ in the upper aquifer is obtained by setting $W T(I, J)$ to the desired head value and assigning $\operatorname{VPRM}(I, J, 1)$ the value 1.0. In the Piceance basin model, the altitude of the stream channel, in feet above mean sea level, is assigned to corresponding elements of WT where hydraulic connection between the alluvium and upper aquifer occurs. Arbitrary values may be assigned to WT wherever $\operatorname{VPRM}(N X, N Y, 1)$ is equal to zero.

Line D24: $\mathrm{HI}(\mathrm{NX}, \mathrm{NY}, \mathrm{NL})$ is an array of initial hydraulic heads. Elements of the array $\mathrm{HI}$ are read, in feet, from card format for $\mathrm{NL}$ aquifers if IRHI has been assigned the value 1. If $|R H|$ has the value 2 , the computer cards which make up the $\mathrm{HI}$ data set must be omitted and the array $\mathrm{HI}$ is assigned values in SUBROUTINE BOUDY, line D34. For steady-state solutions, the initial hydraulic heads may be arbitrary; however, convergence to a solution may require an excessive number of iterations. Therefore, the initial hydraulic heads should approximate the solution as closely as possible. For transientflow problems, the solution depends directly on the initial hydraulic heads which must be precisely defined. The Piceance basin ground-water-flow system is essentially in a steady-state condition so that the steady-state solution (HK) is used as the initial conditions for transient-flow simulations.

The following variables are read at the line indicated in SUBROUTINE RCHRG.

Line E38: $R C I(N X, N Y, N L)$ is an array of uniform recharge velocities. Uniform recharge to $\mathrm{NL}$ aquifers is read in convenient units from card format (table 2). The variables $R 1, R 2$, and $R 3$ are used to change the units of $R C l$ to feet per second. Recharge to the aquifers is considered to be negative. SUBROUTINE RCHRG is called at the beginning of each pumping period but the value of each element of $\mathrm{RCl}$ is assumed to be uniform throughout the period of 
simulation. Consequently, the recharge rate cannot be varied without modification of the program. In the Piceance basin model, uniform recharge from precipitation is assumed to occur only in the upper aquifer. Therefore, only the elements of $\mathrm{RCl}(\mathrm{NX}, \mathrm{NY}, 1)$ are read into the model and the elements of $\mathrm{RCI}(\mathrm{NX}, \mathrm{NY}, 2)$ are assigned the value zero in the program.

Line E57: NREC is the number of pumping nodes to be simulated during each pumping period. If NREC is greater than zero, the program will read ( 1 ine E61) NREC computer cards (table 2) containing the location (IX,IY,IL) of the pumping node and the pumping rate (RCE) for that pumping node. A data card specifying NREC followed by NREC data cards defining IX, IY, IL, and RCE must be included in the data set for each pumping period. In the Piceance bas in model, no wells are simulated in the steady-state solution and a value of zero is read for NREC.

A listing of the computer program used to simulate steady-state groundwater flow in the Piceance basin follows. A flow chart is included for the main program and each subroutine to aid the user in understanding the program. The program listing includes the input data set and a copy of the printed output. The hydraulic head distribution given in the output is the steadystate solution which is used as initial conditions for transient-flow problems. 


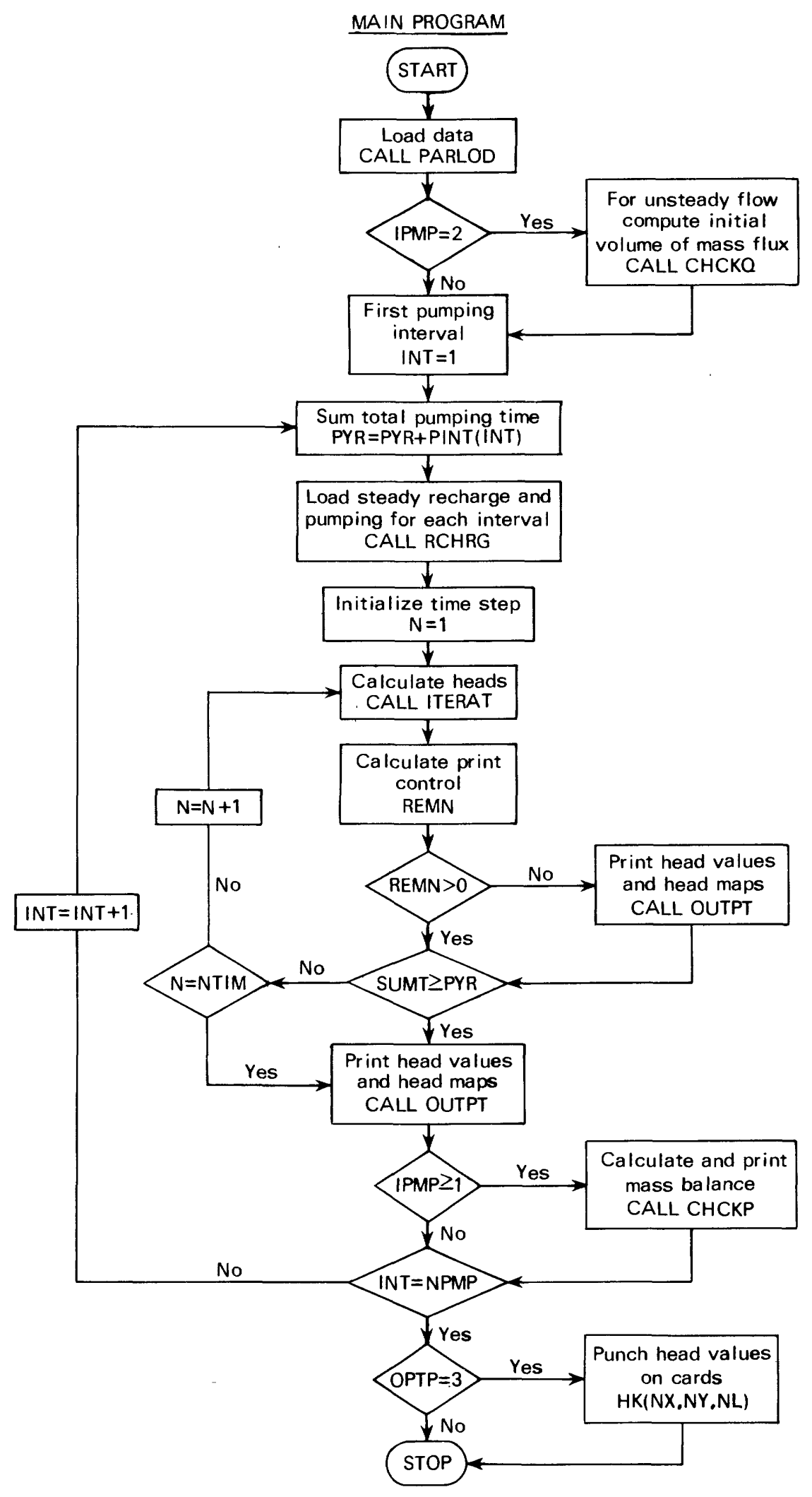




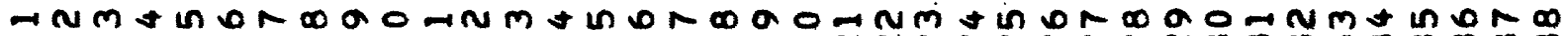

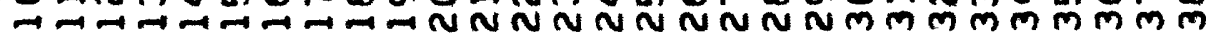

$<<<<<<<<<<<<<<<<<<<<<<<<<<<<<<<<<<<<<<<$

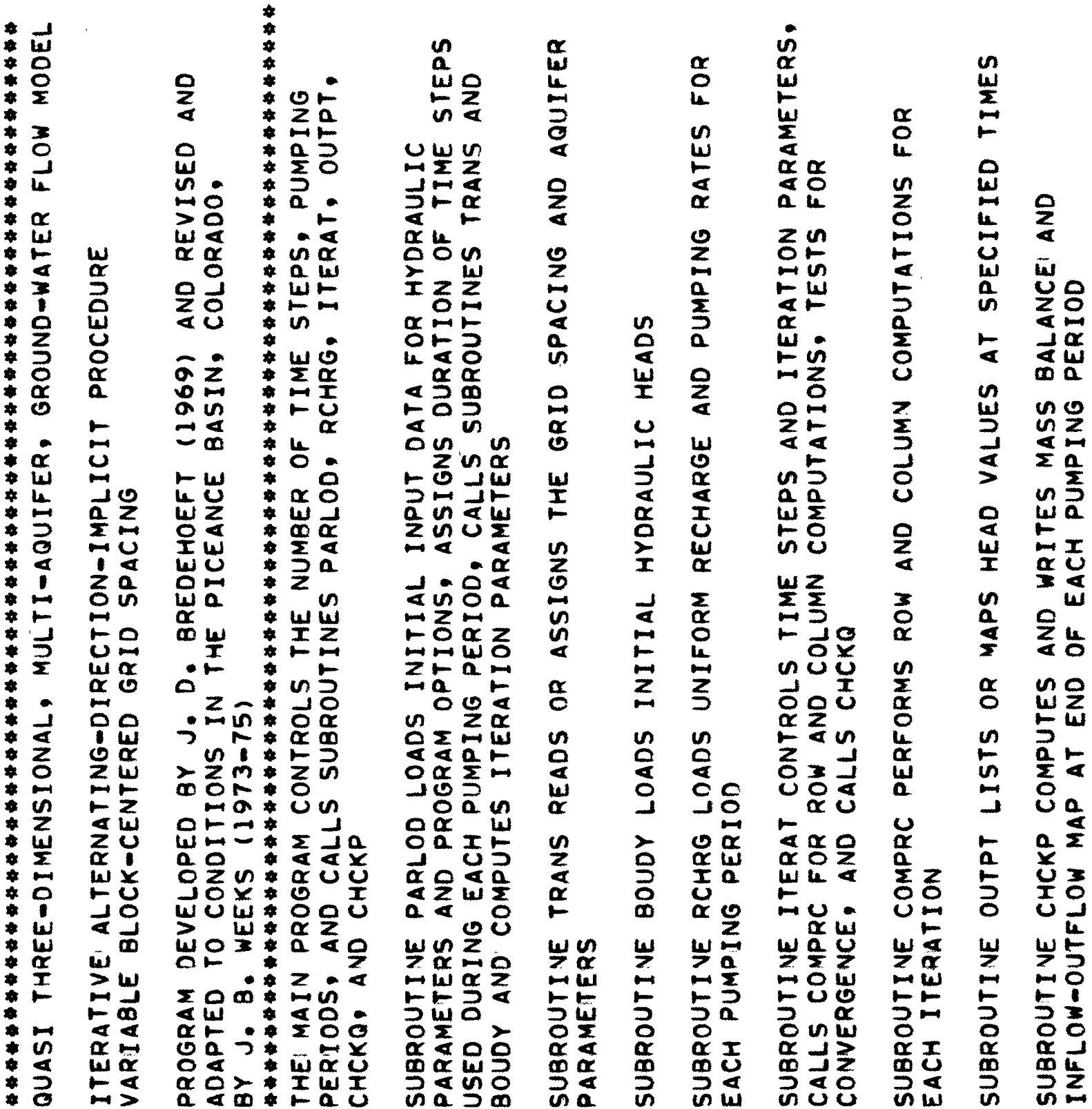




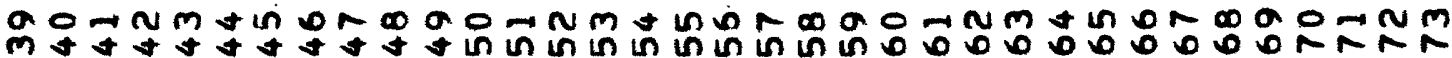
$<<<<<<<<<<<<<<<<<<<<<<<<<<<<<<<<<<<$

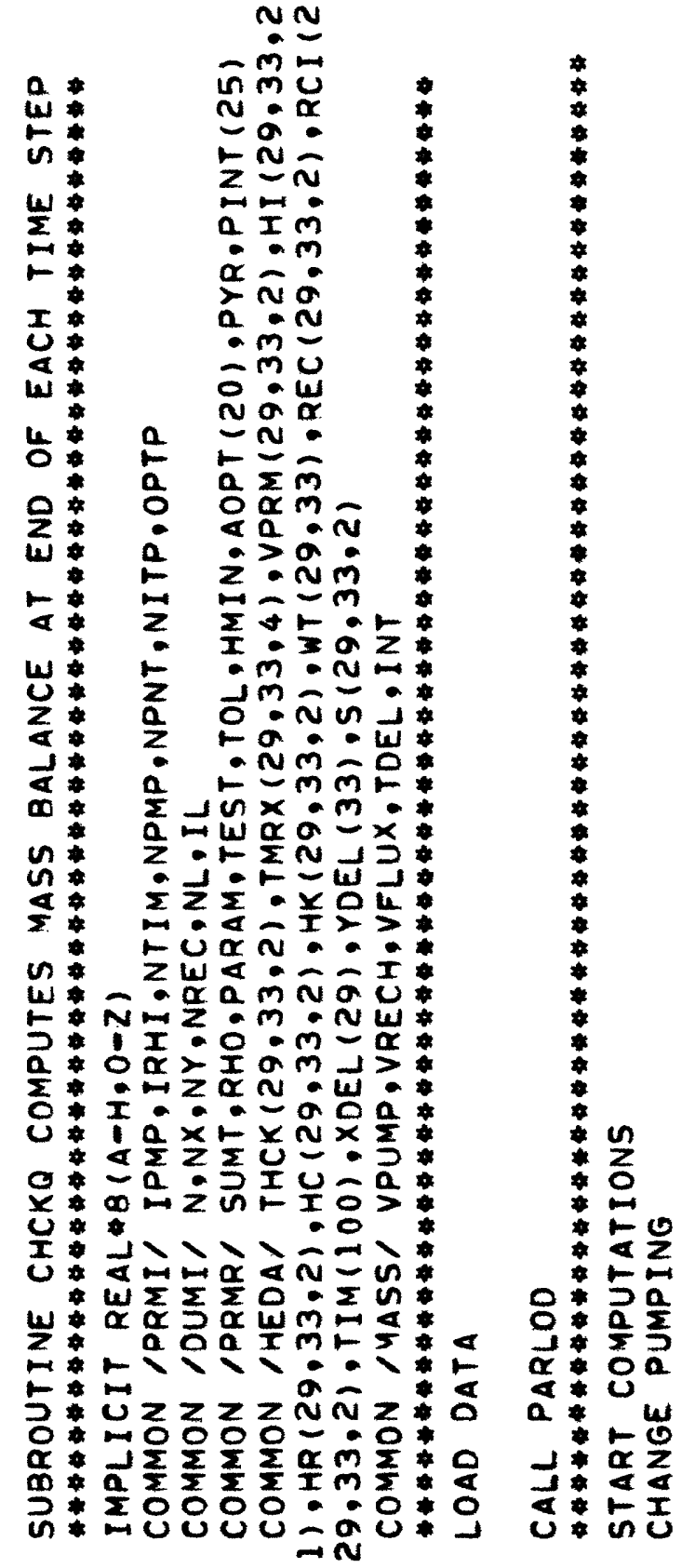

\begin{tabular}{l}
0 \\
$\frac{1}{U}$ \\
\hline
\end{tabular}

$\frac{1}{4}$

40 $\frac{a}{2}$ a N $z$

- 1 $0 \rightarrow z$ w $n$ $a \geq z$ $\frac{1}{2} i$ a 2 $\rightarrow 0 a$ $- \pm \frac{1}{a}$ $40>$

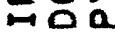

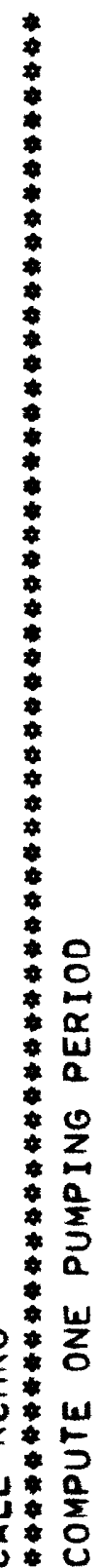
안 88 $=0 \quad \alpha$ $\sum a_{i}^{2} 0^{2}$ $z-z=0-\infty$ $-\alpha=0$ แ $10 \geq 1-\omega$

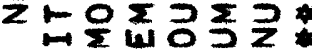

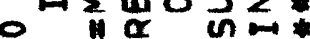
$N \pm \frac{2}{2}-1-1$

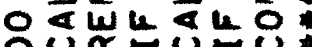
00 
- nonadomnmtunt

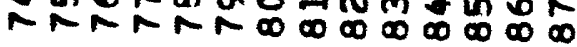

$\alpha<<<<<<<<<<<<<$

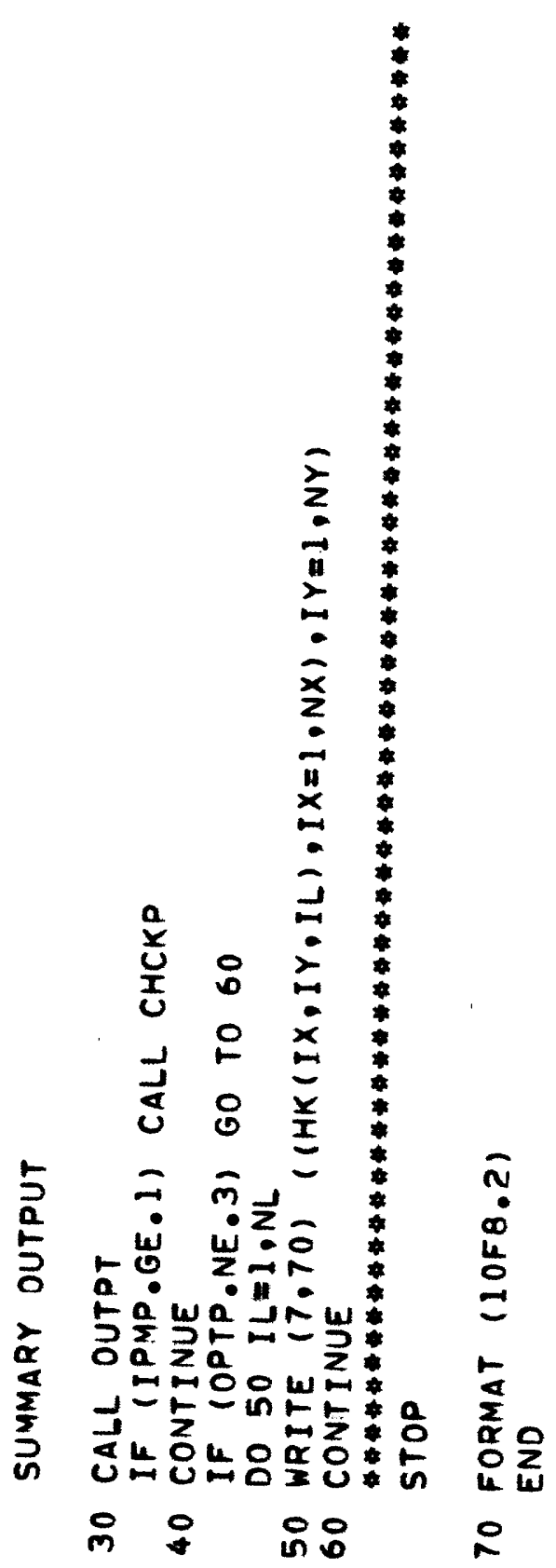

UU U U 
SUBROUTINE PARLOD

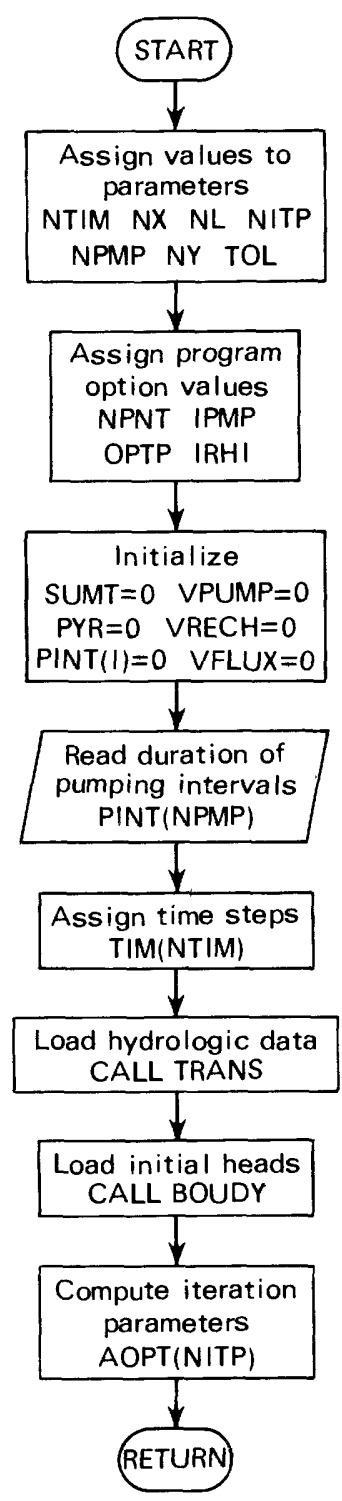




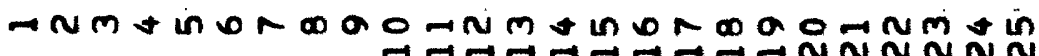

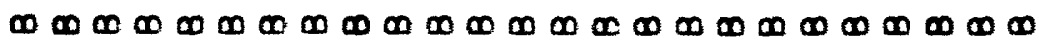

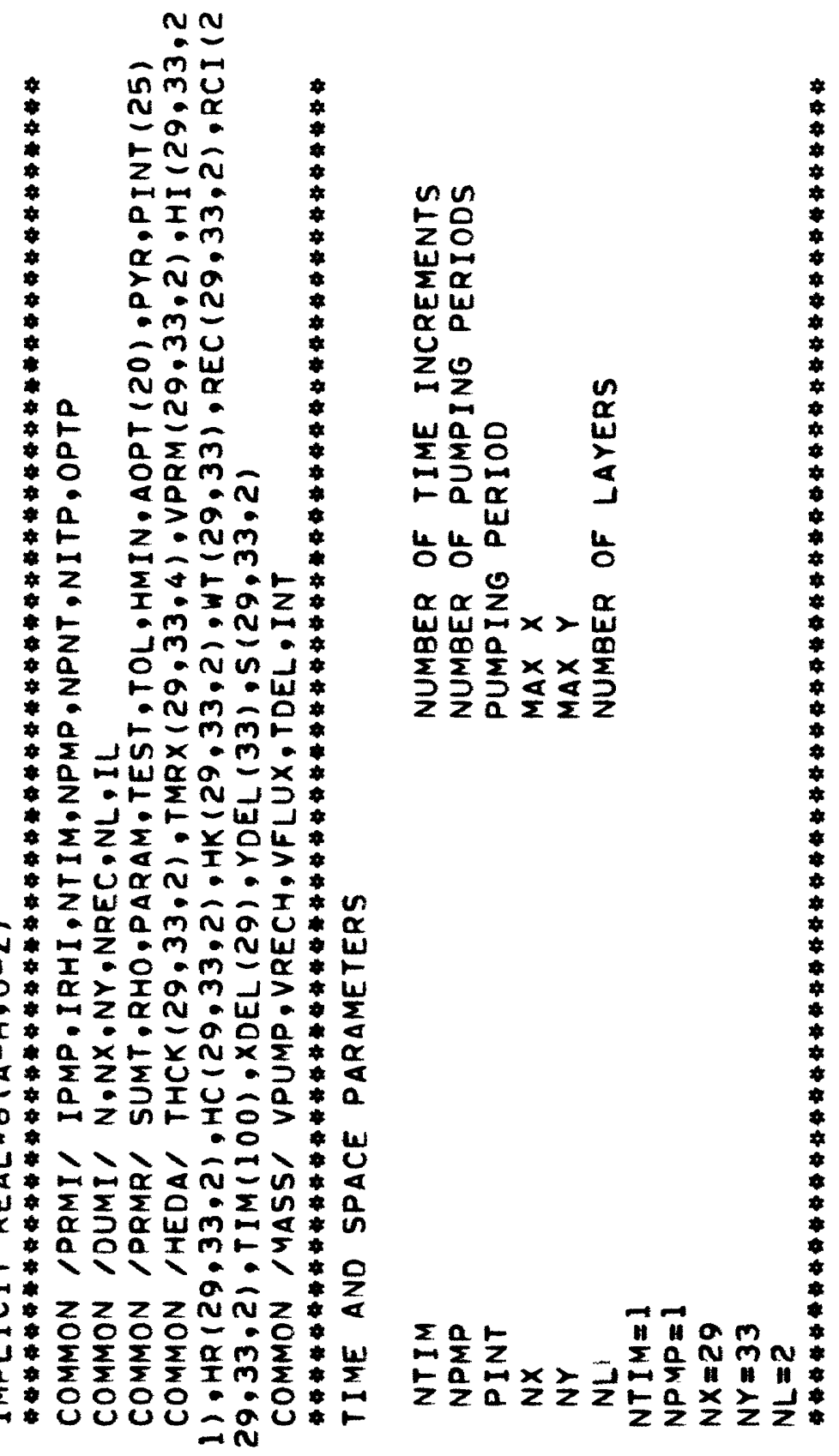




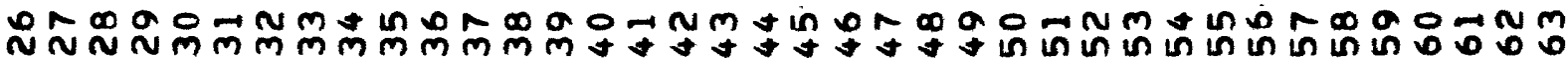
ח ח
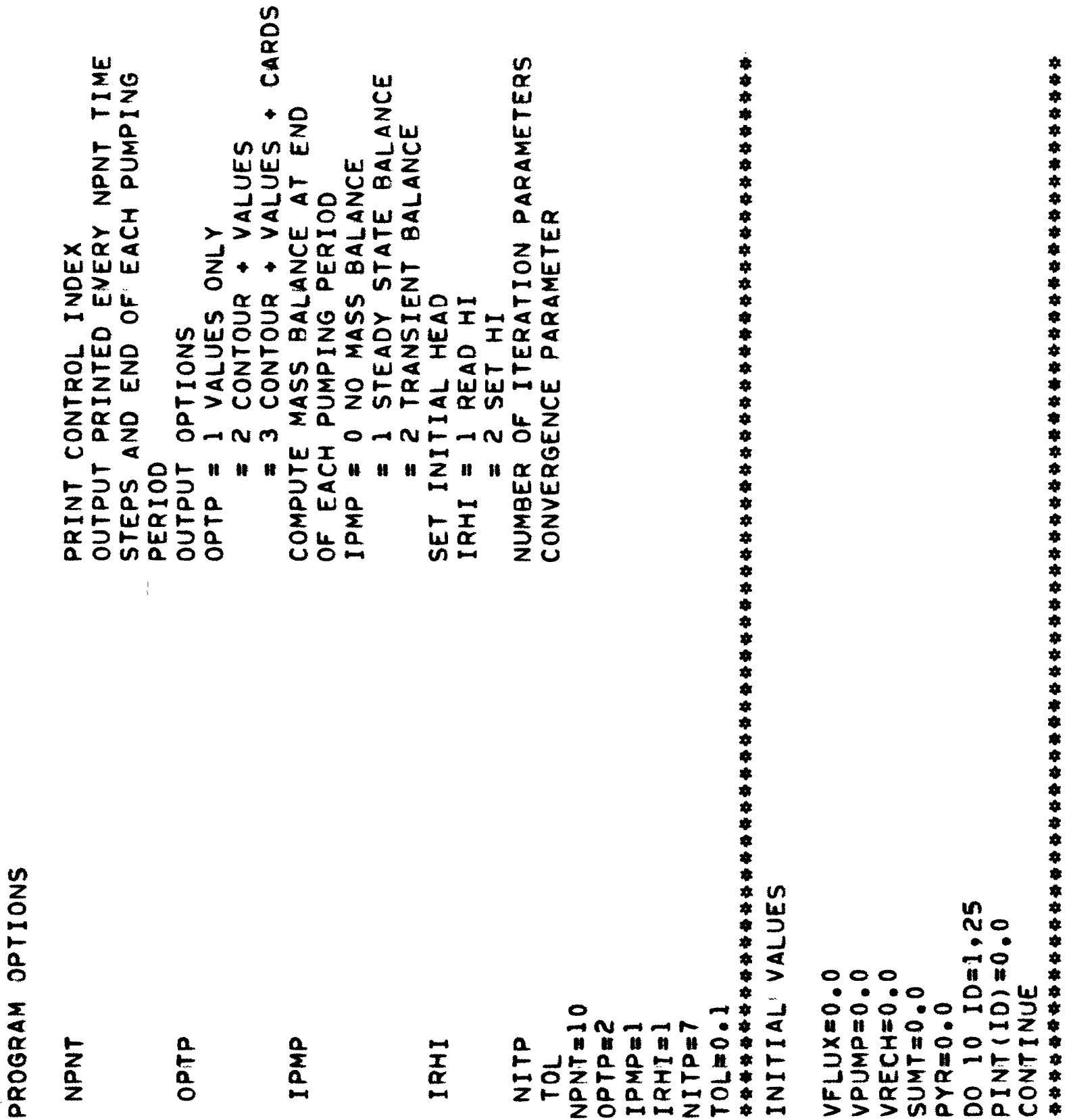

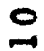




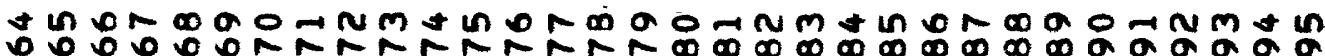

n

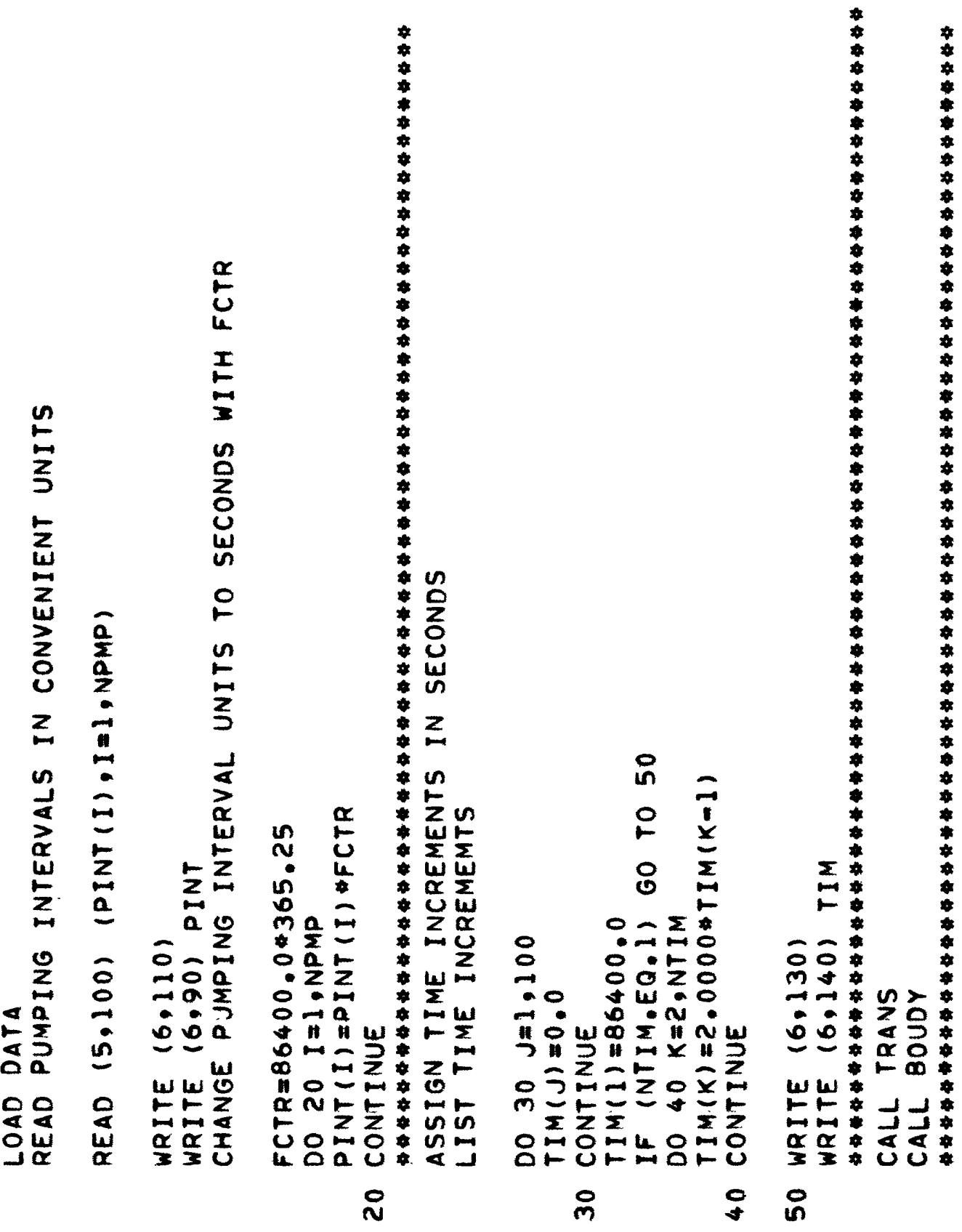

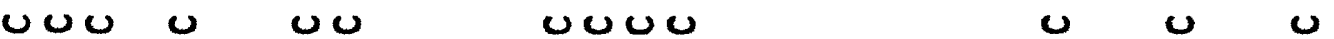




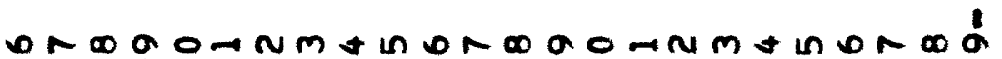

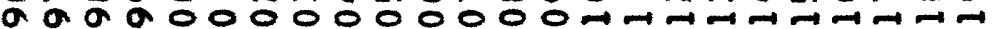

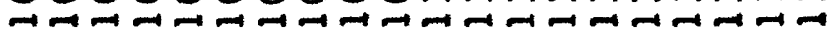

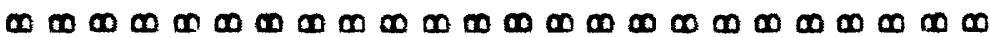

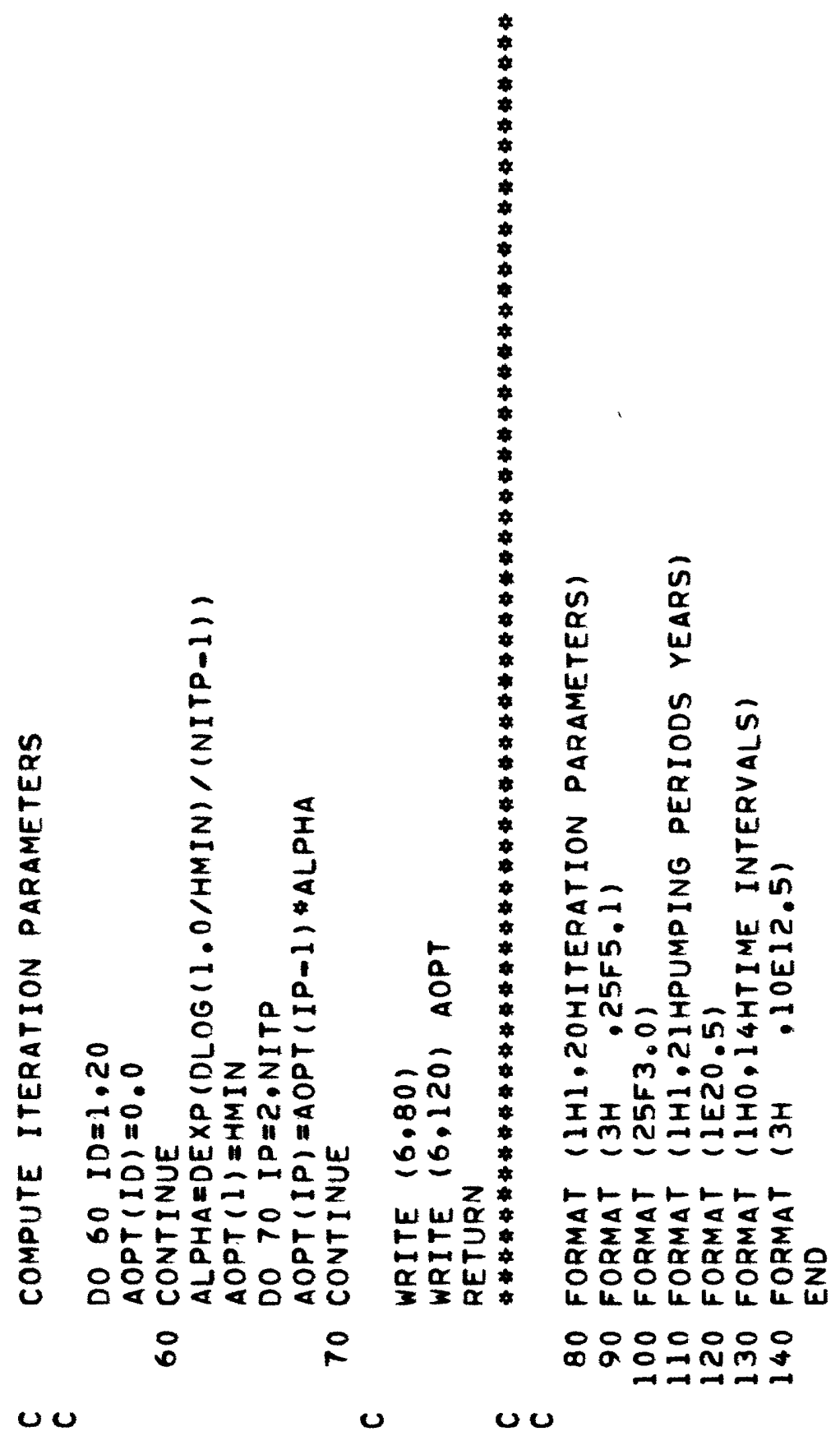




\section{SUBROUTINE TRANS}

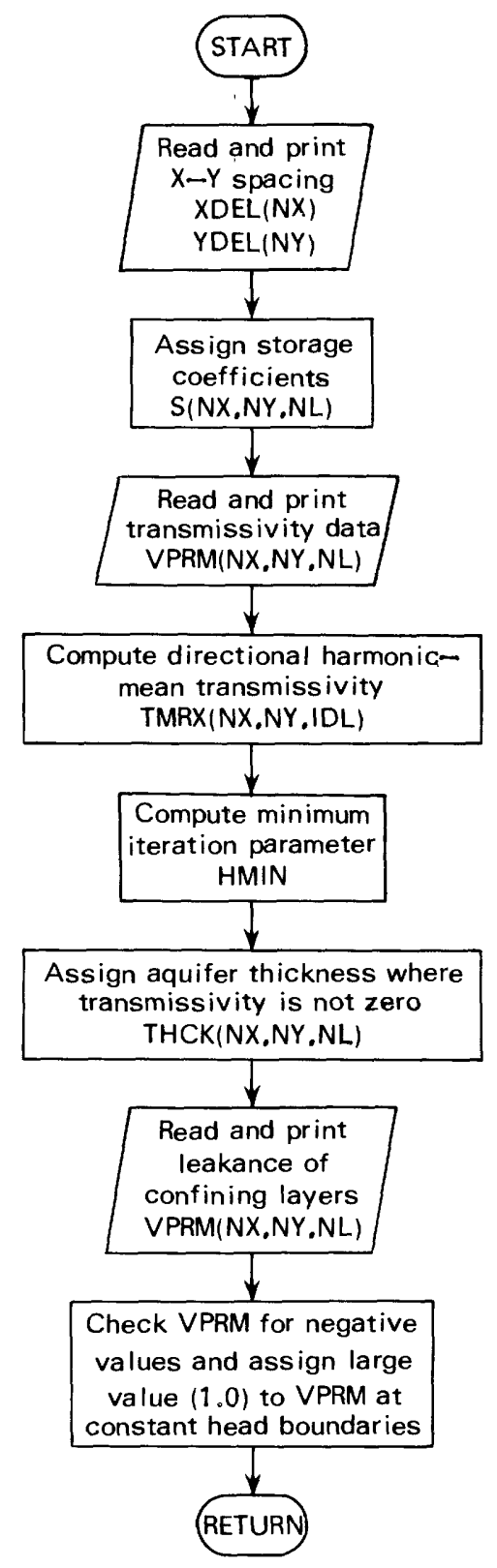




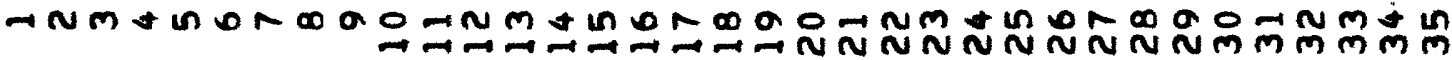

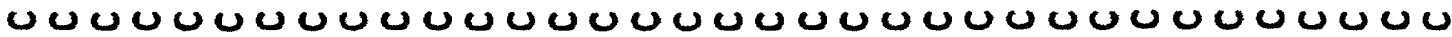

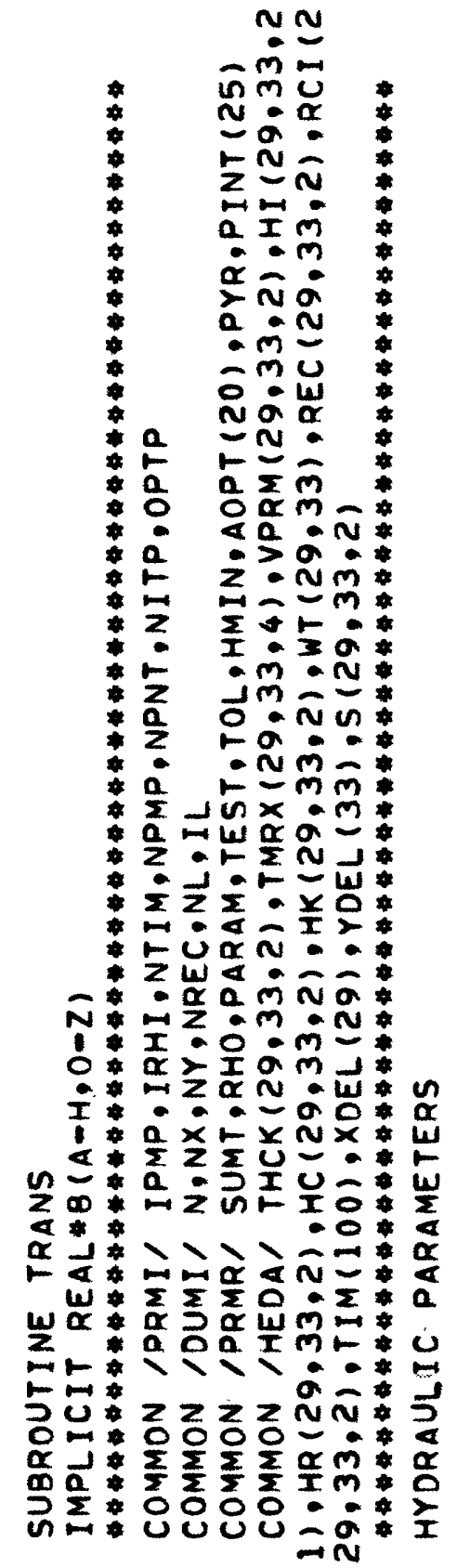

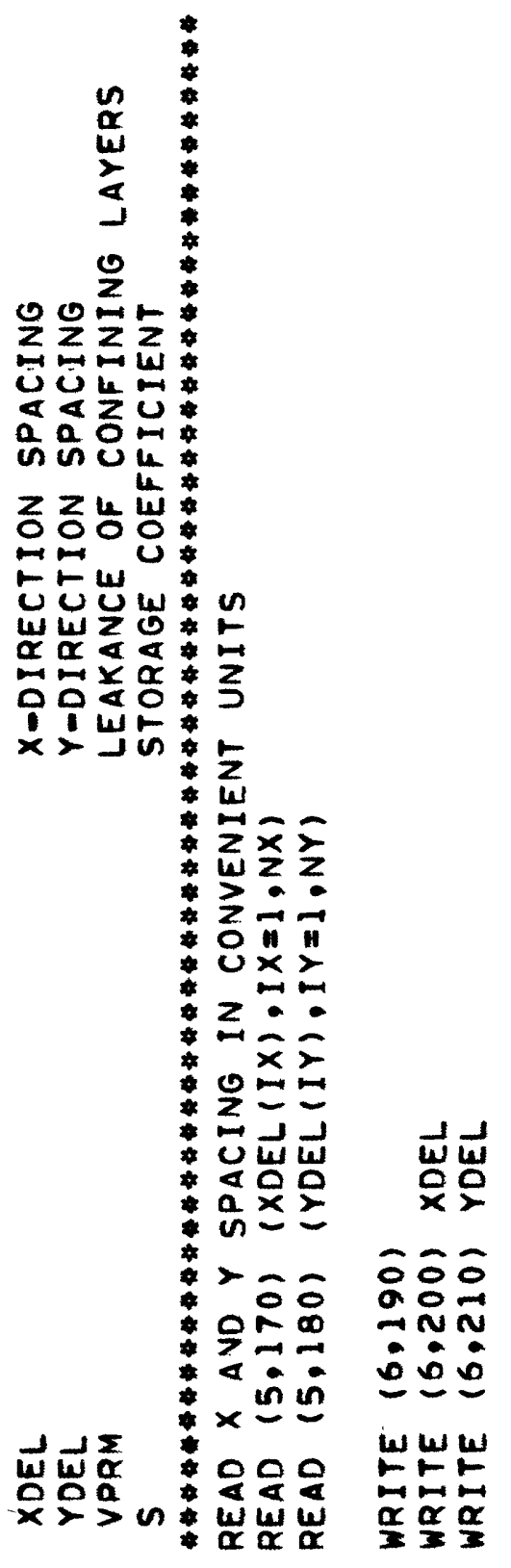

$a$
0
5
5
5
$w$
$w$
$w$

出

L.

0

$0_{0} N \quad$ $20 \times 2$ $\rightarrow \sum \omega$ $00-0 \quad-0$ $4 \cdot \rightarrow x$ a o in N $x \geq 3$ $\omega_{1}$ in 0002 난

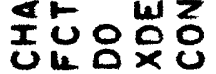


ON

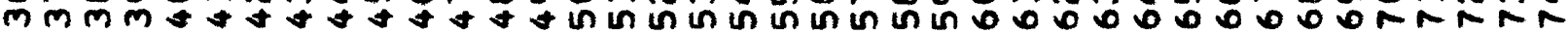

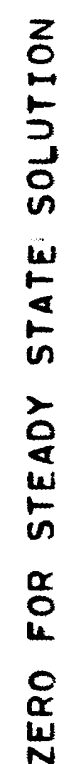

$\sum_{n-1}^{n}$

w5

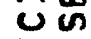

in

is

w 2

엄

U분

岁出

\&u

뚱

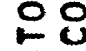

$\overrightarrow{10} \mathrm{n}=$

$2 \omega$

0 \&

봉

ํํㄴ

\& in

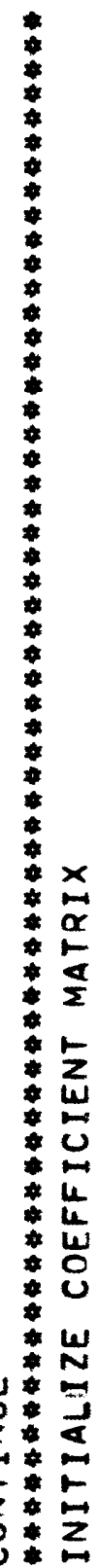

m

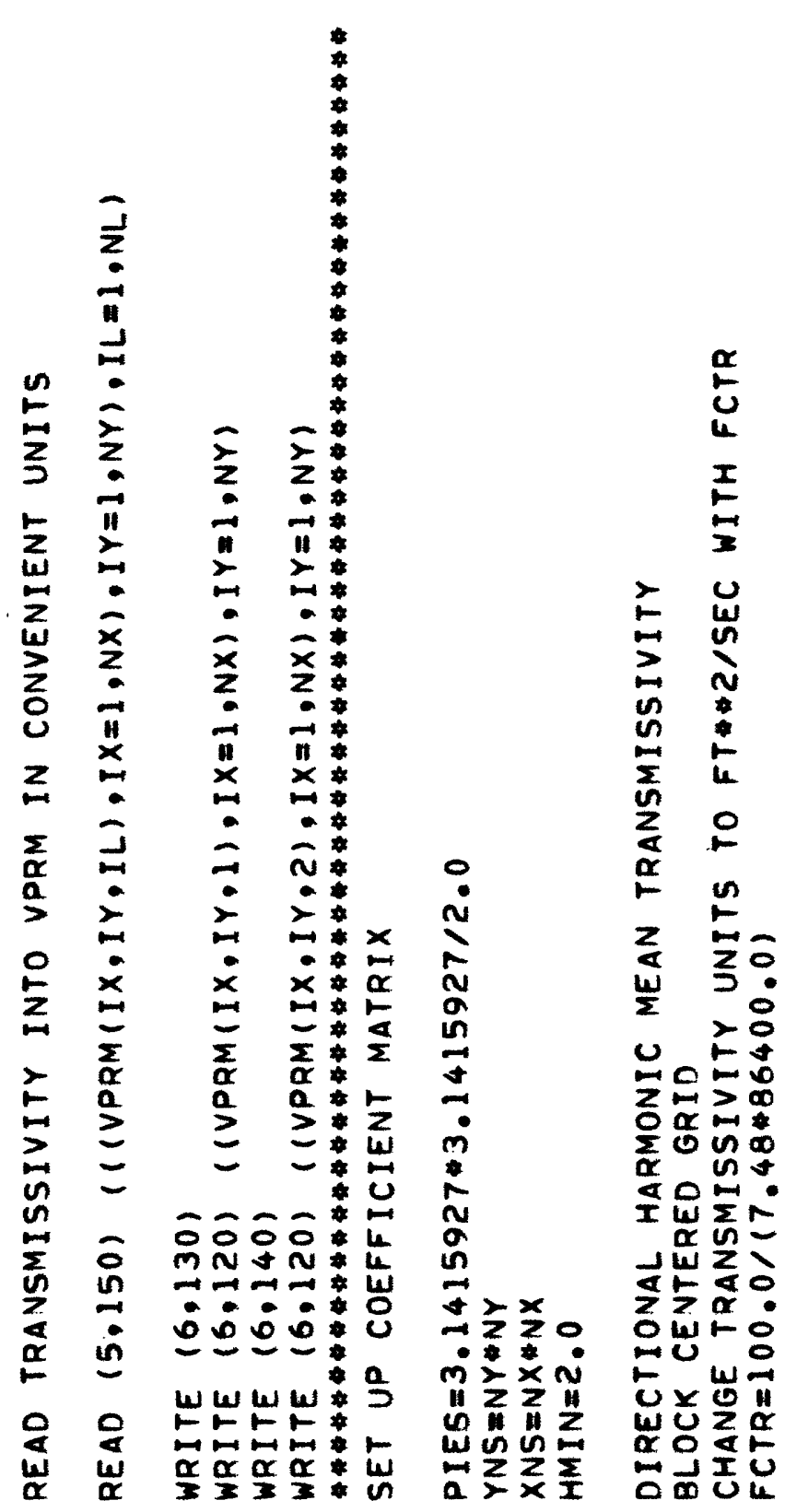

$+$ 
nONrRara

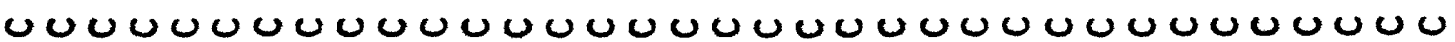

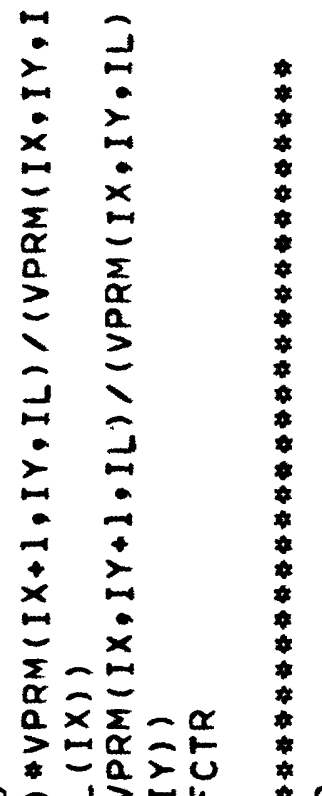

$0<1>11$

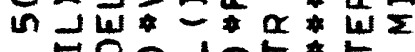
$\because 0-1=5 *$

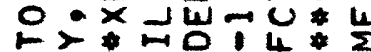
$2 \div \div 0 \%$ $0 \div-1>+\infty$ $0 \times-2+0$

$\rightarrow-\infty>0$ $\widetilde{0}-x=1-1$ $0 \geq m-2-z$ - $a-2-\infty \times 10$ $0-2 \leq+\cdots$ $->-\alpha>x+1$

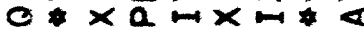
$40 m>a r<\alpha$ $-1 \times 2 \times 24$ $-N=0-1-1$ - I $\alpha \cdot-\| \Sigma \Sigma$ $m-a \mathrm{~N}=1 \%$

$-\rightarrow>\| \frac{\alpha}{\alpha} \rightarrow n$ $>x>1+2 a+2$

$\Rightarrow z z-0-0>00+\frac{2}{2}$

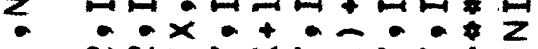

$-N \times m>x>-15$ $-1+x=0,0<0$

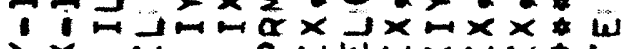
$\geq x \rightarrow$ a $2 z 0+00>-0-1-2+0$ in in $x$ in $-x \times x$ u $x \times 2$

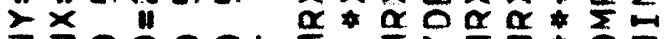

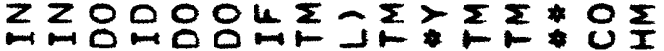

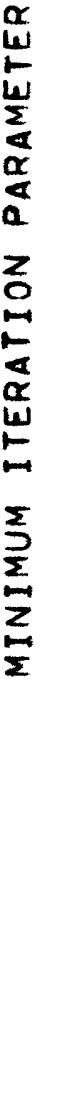

in

$0 x$ $0 \ln \frac{\alpha}{2}$

0

$8 \vdash$

$-0>$

6 -

$0-1,4$

- $a x>$

$000=0 \sum \Sigma$

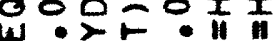

- $0 * 4-z z$

$-4 \sim \alpha \sim-m$

$\because \div \div 0$ -

000.

머들

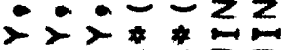

mm Un

- * $z Z I I$

$x \times x \times \geq \div$

$m m=-1$

$x=x$ in in

$\alpha$ a $w \omega x>$

$\Sigma \frac{2}{\Sigma}=\omega \sum \Sigma$

$-1-a$ a I I

$-2 \| n=2$ $-x>$

uk a $\sum \sum$ u $m \omega \alpha I I m \omega$

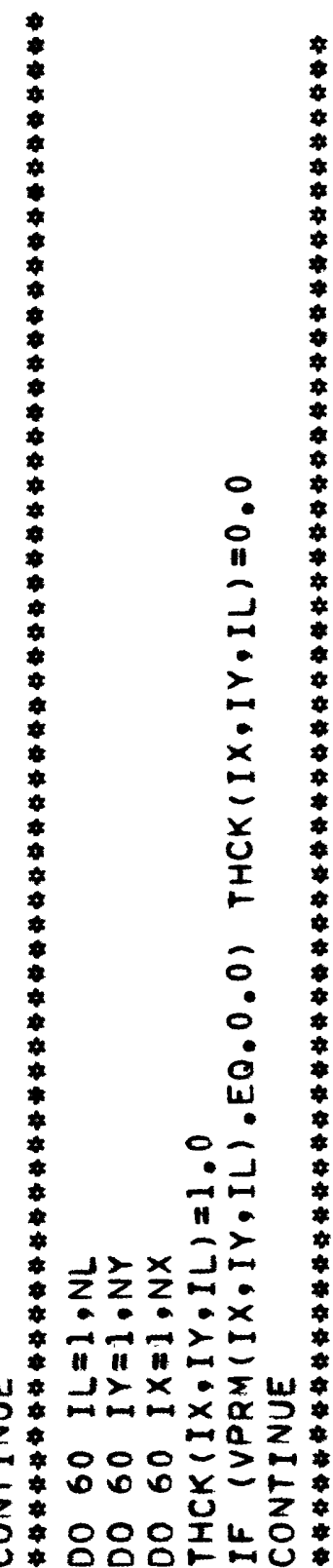

in

$$
0
$$




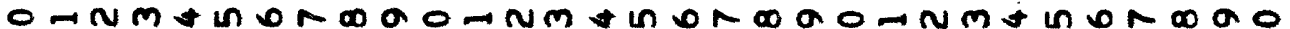

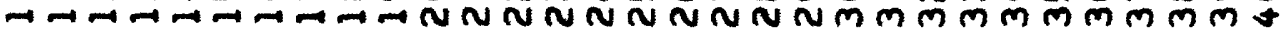

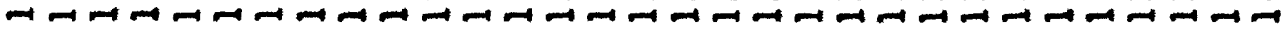

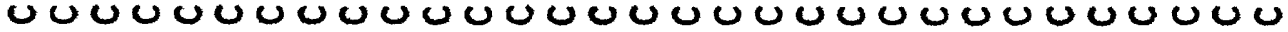

年

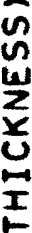

$\checkmark$

$>n$

$-\infty$

$\rightarrow 2$

a)

\&

$w z$

$\sum_{\alpha}$

wa $z$

a.

32

$\cup \mathcal{U}$

$\rightarrow$

52

w

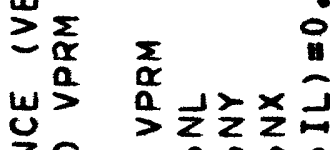

$\sum_{0} \quad \omega \div-\div ;$

\& $\quad w \vec{n} \vec{u}=$

$a m-1>x \rightarrow \omega$

w $15 m m \times 3$

lu 2000 m

OU $-N R N=$

기 $\Rightarrow 000$ 年

wa $w_{1} 00000$

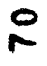

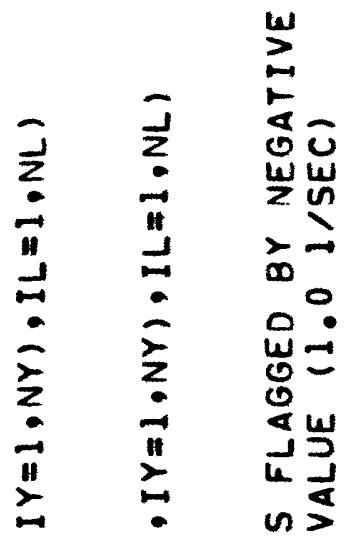

a w

$-0 k$

00

To

E⿻

mw

II

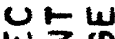

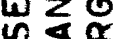

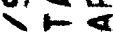

U

$0 \sum_{0}^{2}$

$\rightarrow$

n 4

$-6 \mathrm{n}$

$2 z$

02

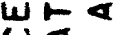

乐它

जo $\quad \begin{aligned} & 2 \\ & 0\end{aligned} \frac{2}{\alpha}$

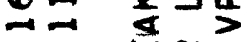

iv $\alpha$

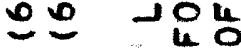

wh $0 \times 4$

- 1 zU⿺ 一

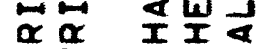

$\boldsymbol{I} \boldsymbol{U}>$

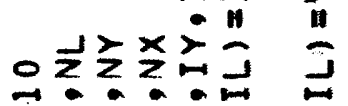

i $\rightarrow-x<$ -

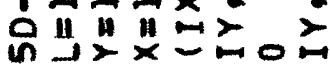

n $m m \sum-0 \cdot \mu$ -1000 a $0^{\alpha}-x \geqslant$

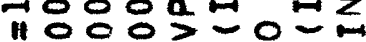

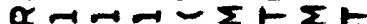

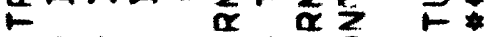

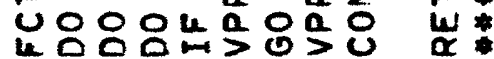

$$
\begin{array}{lll}
0 & 0 & 0 \\
0 & 0
\end{array}
$$

$\cup \cup$ 


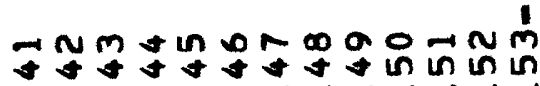

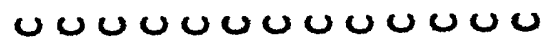

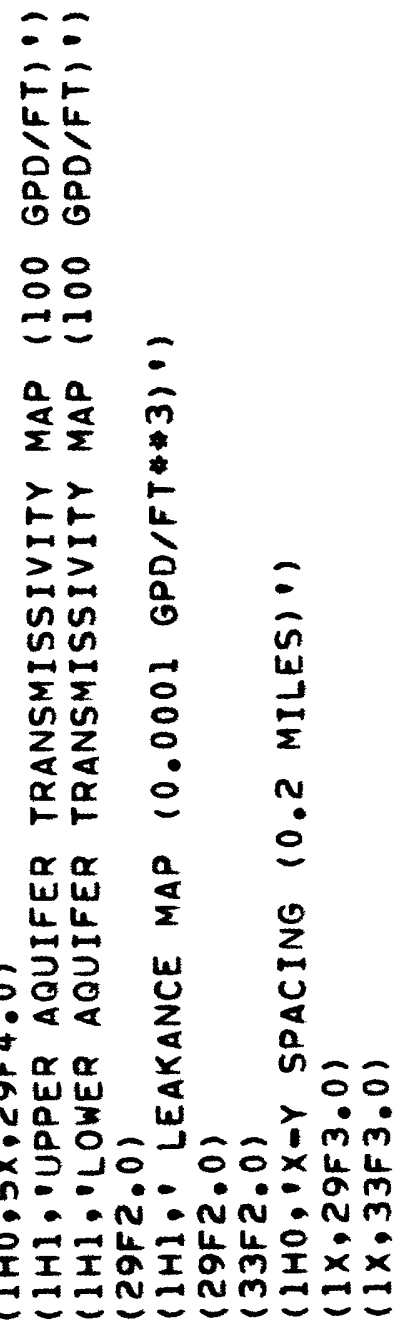

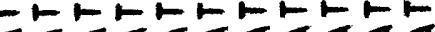

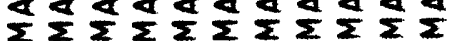
$\alpha \alpha \alpha \alpha \alpha \alpha \alpha \alpha \alpha \alpha \alpha 0$ 000000000002

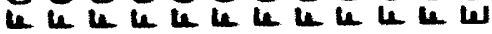
웅ㅇㅇㅇㅇㅇㅇㅇㅇㅇ a $M+\ln 0$ -

$\omega$ 
SUBROUTINE BOUDY

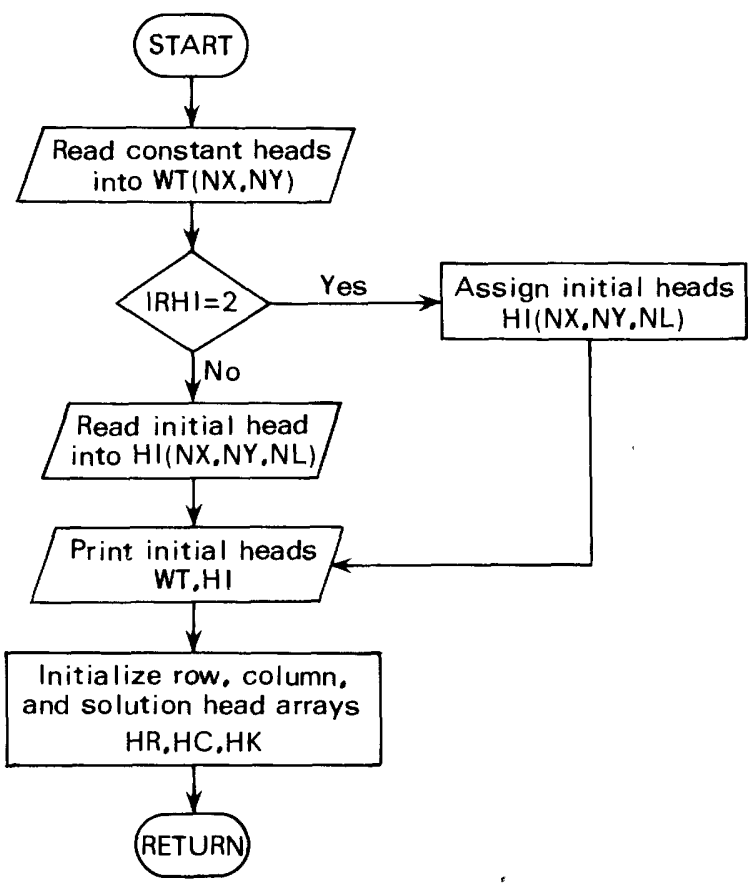




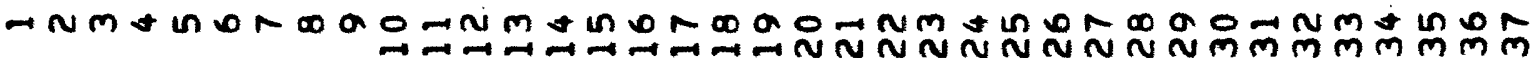

N

$m \rightarrow$

$\ln \omega$

$\sim a \underline{\alpha}$

Z $\widetilde{\mathrm{N}}$

$m m$.

a Im

- $m$

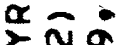

a $-\mathrm{N}$

- m-

mu

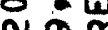

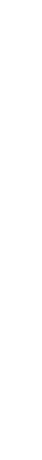

$\frac{1}{a}=\frac{N}{0}$

- o oam-

- a a r.

- a $->a$

- $-z a n m$

* m $m 2$ -

- Z I \pm 10

- $I-x a$.

* $-m \cdot N$

* z

* z $-a-\ldots$

* anm-

*a $\leftarrow$ Inm

- $\Sigma-u \times a m$

- a mua $a-$

* Z-r $\mathbf{I} \mathrm{N}$

+ Jar-u.

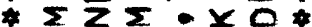

- $-4-I>$

* tuan - .

- zua. - -

- a a man

$N * m Z \cdot m-N$

- I $-0 \cdot m$.

$0 * \bar{\alpha}>I a m$,

- $-z \alpha N-u * u$

I $*-200$

1 axpxnx u

\& $\Sigma \geq \sum \omega-\ldots$

$>-a$ a

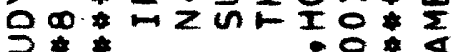

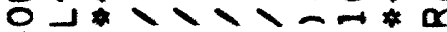

$\infty<* m \alpha \alpha \hat{N}=* \alpha$

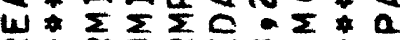

u $\alpha * \alpha \geqslant \alpha \omega m$

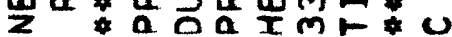

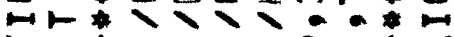

一U

$0=00000$

$\alpha+\sum \sum \sum \sum \alpha m$

क a

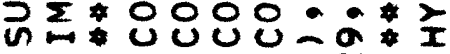
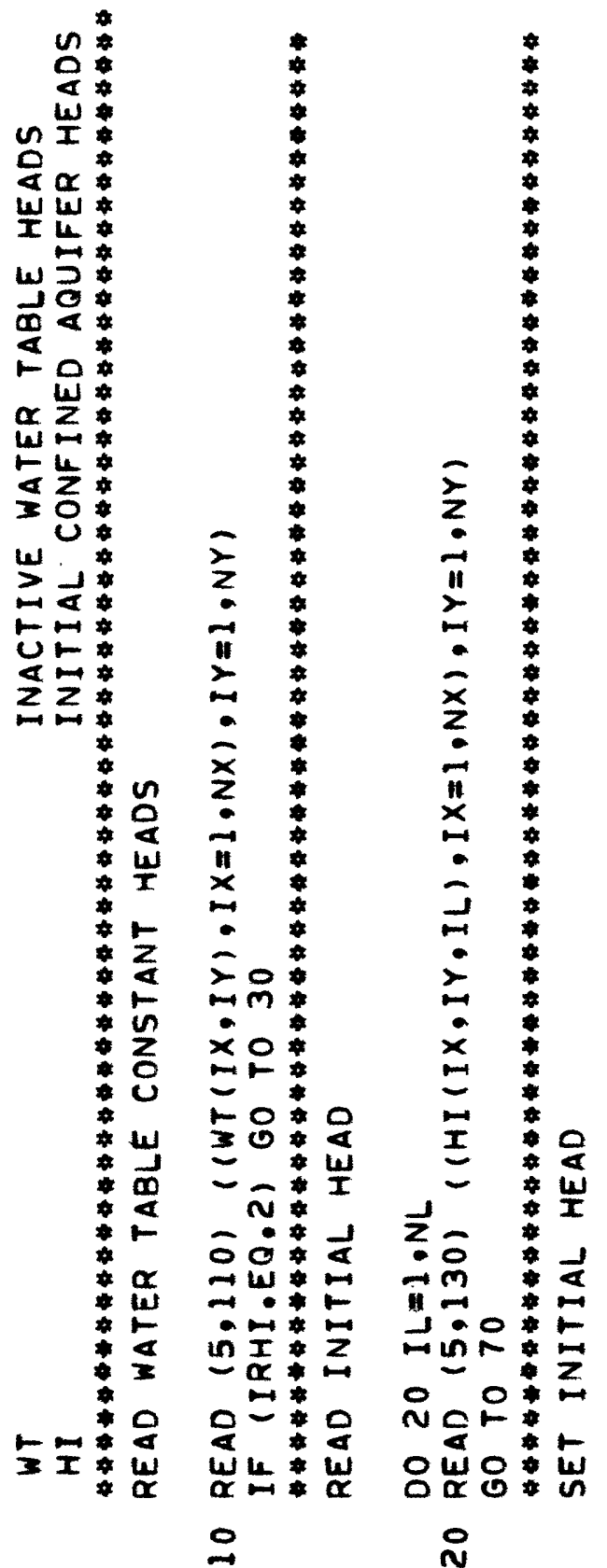

8

\&

o

$\infty$

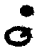

u.

- 0

70

$=0$

$-0$

$>-1$

$1>x-n$ n

$z_{0} z_{0} \div \ldots$

$\therefore \div \dot{m} \vec{m}$

in in 1 - $\circ$ a

$-2 x x>0>\omega$

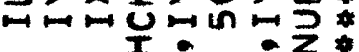

$000=x 0 \times m$

in $n$ in $-m+15$

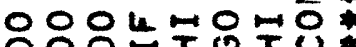

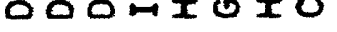




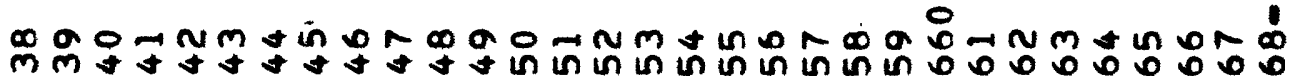
0000000000000000000000000000000

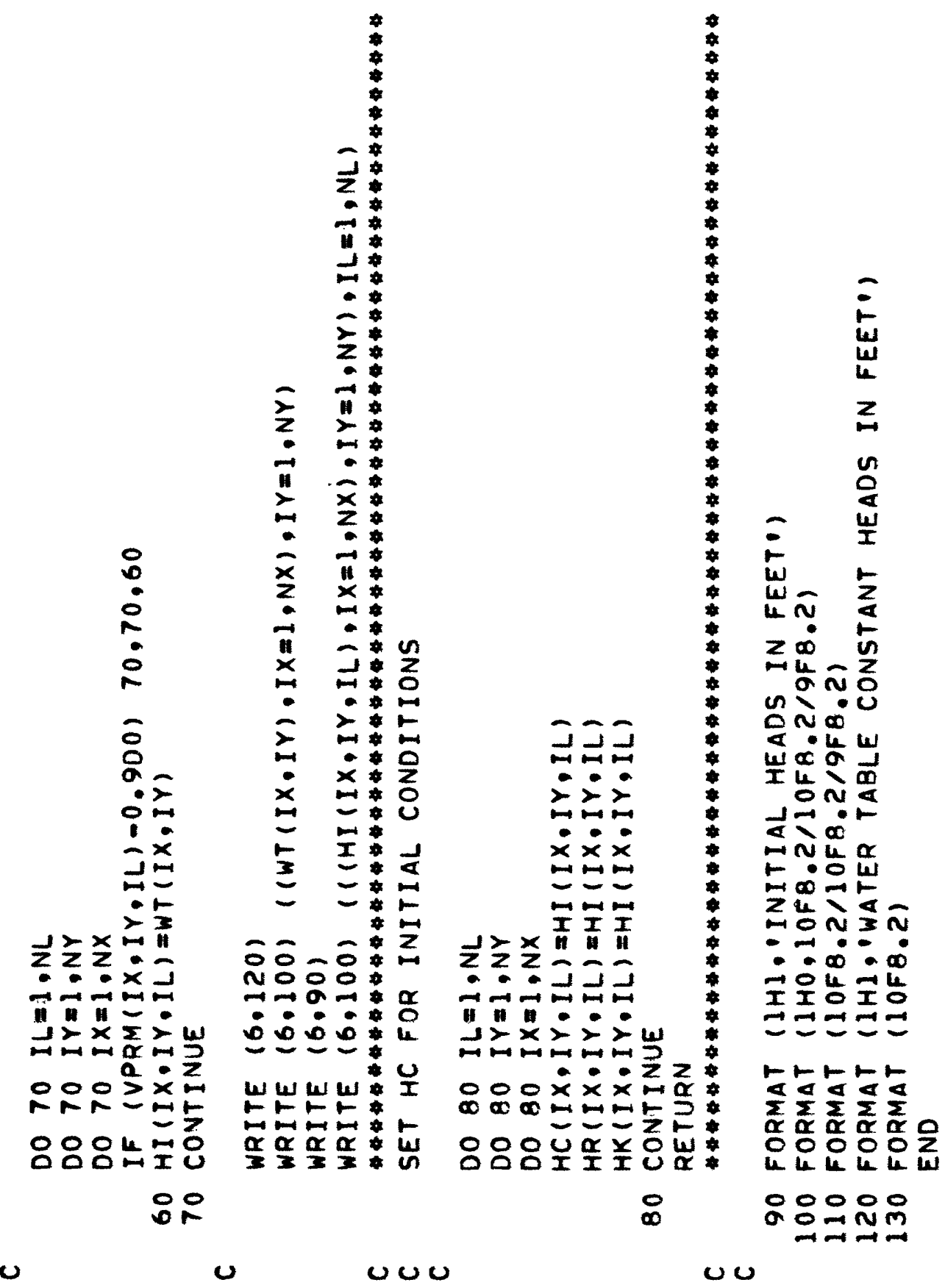


SUBROUTINE RCHRG

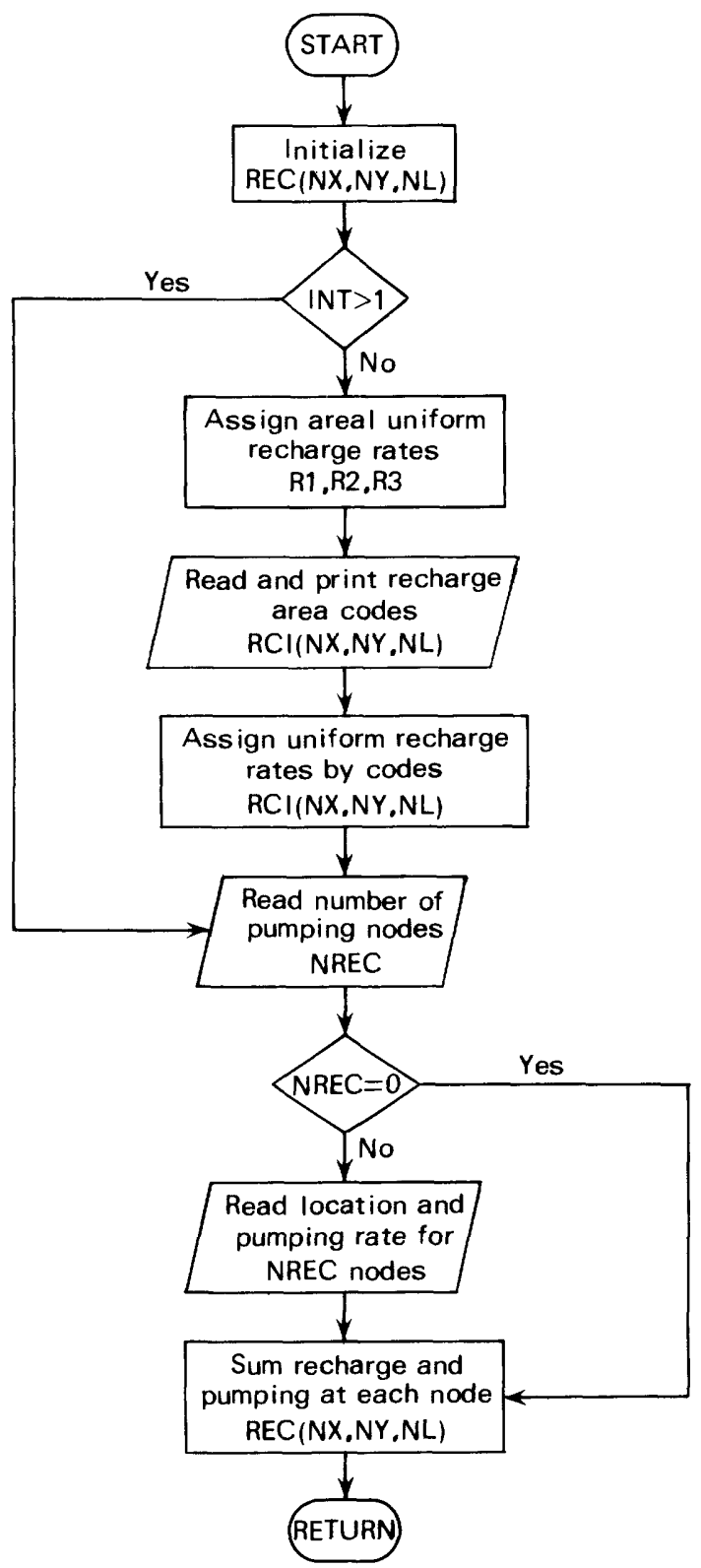




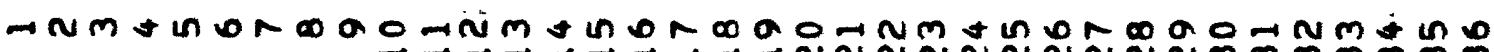

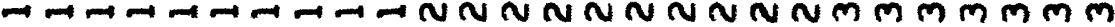

|

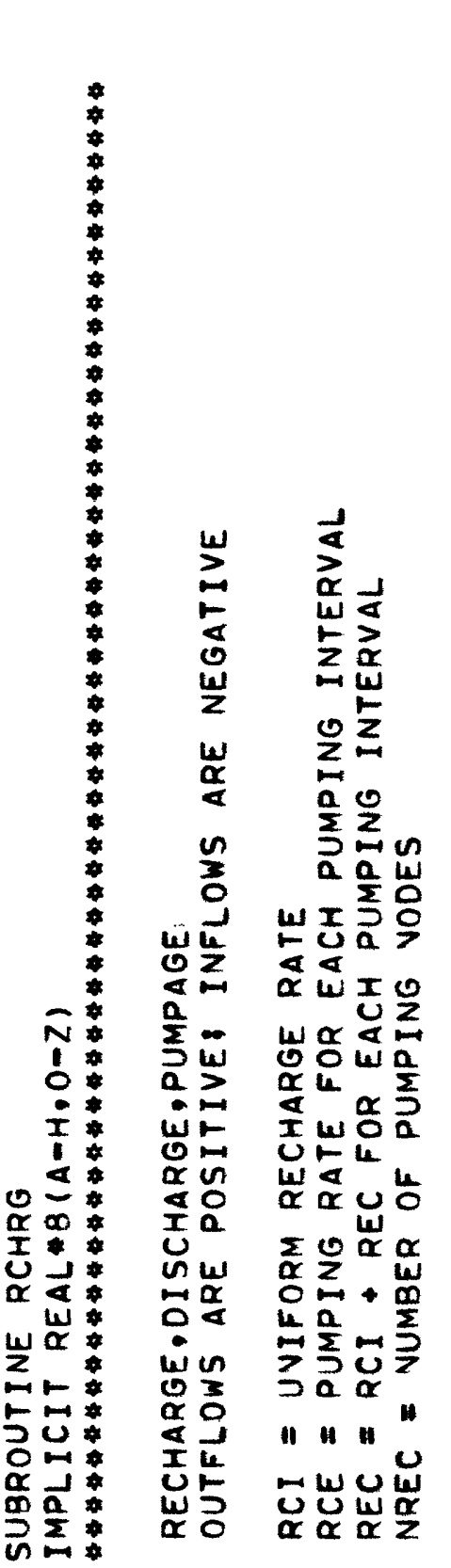

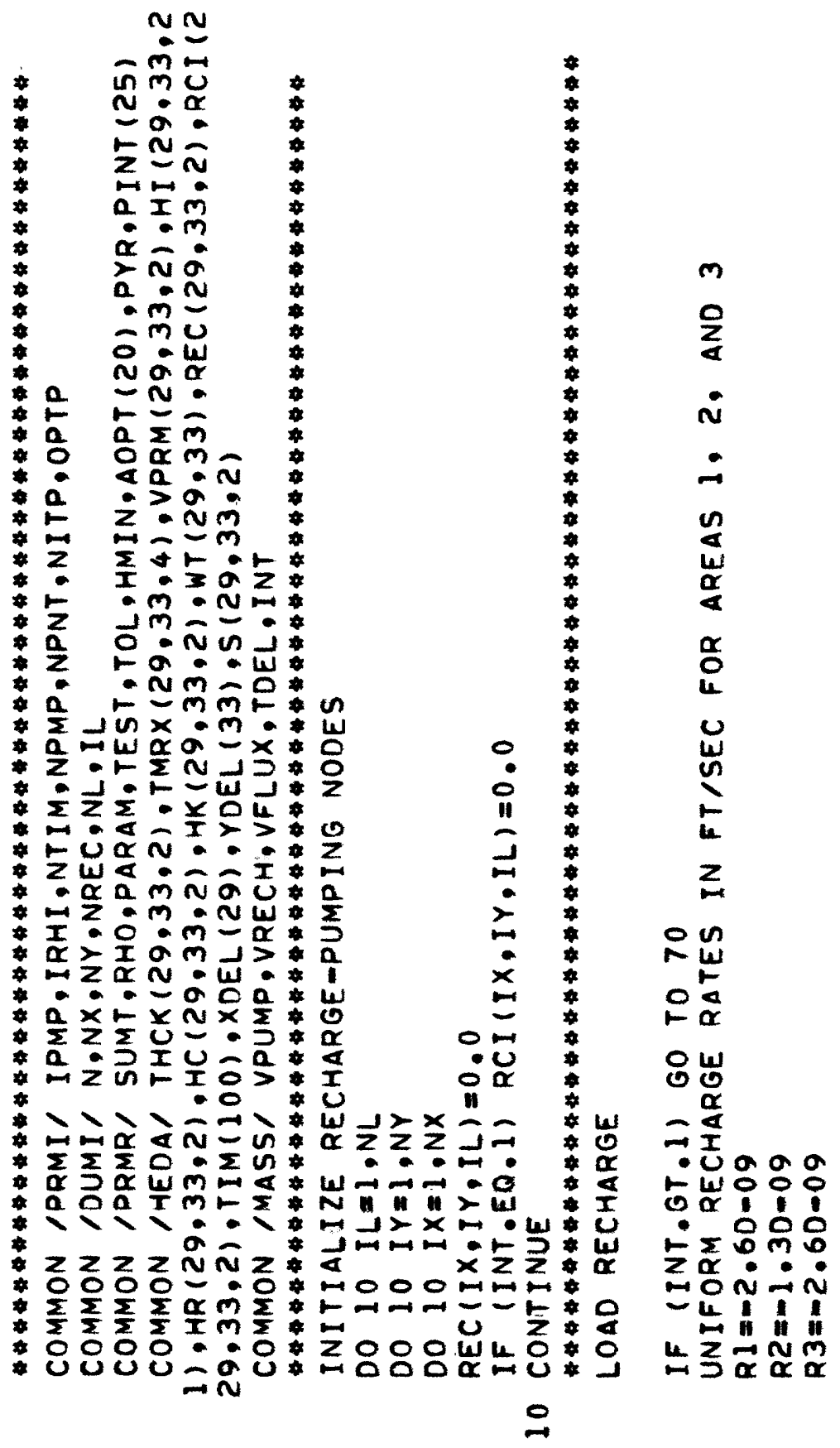

$\boldsymbol{\omega}$

$\omega \cup \cup \omega$ 
n

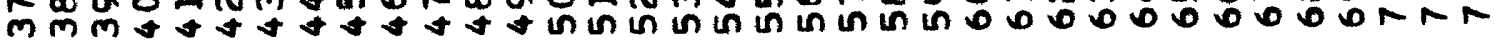

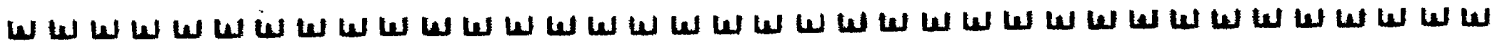

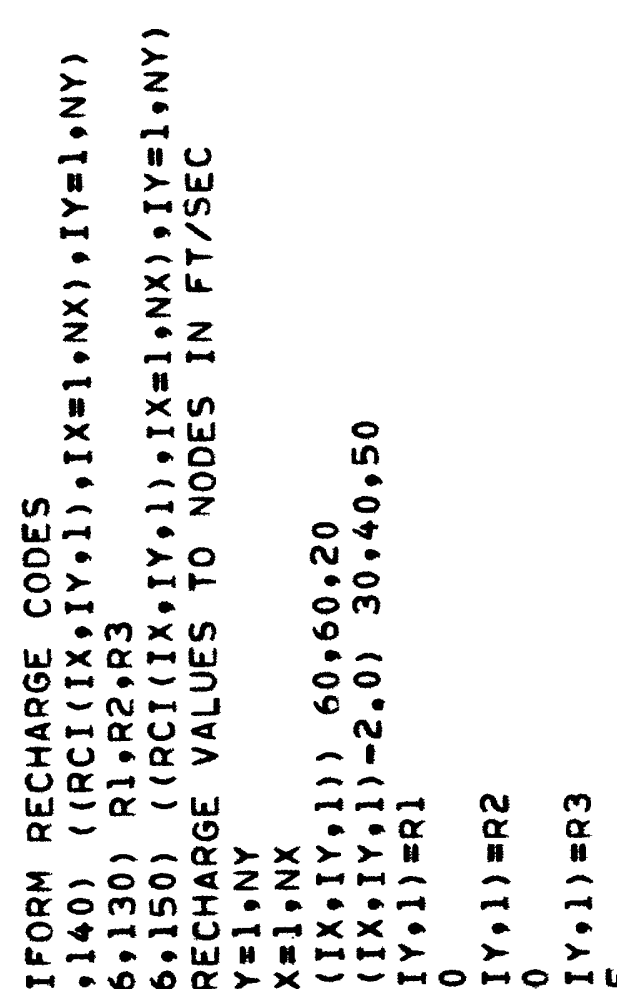

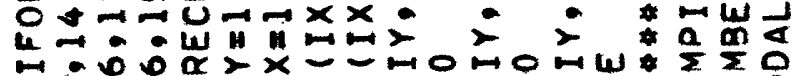

zin

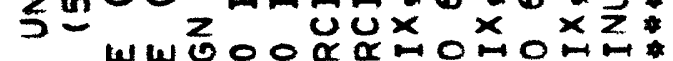
(1)

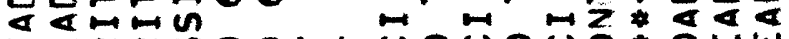

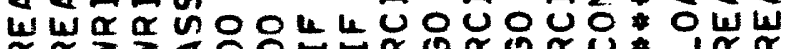

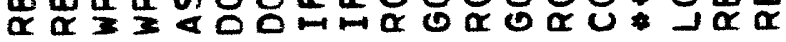

$$
\text { No } \& \text { in }
$$

$n$
$w$
0
$z$

$\omega$

$\frac{w}{2}$

$\frac{\alpha}{0}$

n

$z$

$\underset{5}{m}$

$\omega \alpha$

$\alpha$

$\geq 0$

n

$4 \geq$

20

$0 \frac{0}{2}$

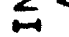

a $z$

5

4

100

0 $2 \geqslant 2$ 0
$\alpha$
$\alpha$
$\alpha$ (a) 
$m+n \infty N \infty a 0 \rightarrow n \dot{m}$ NRNRRR⿻

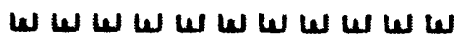

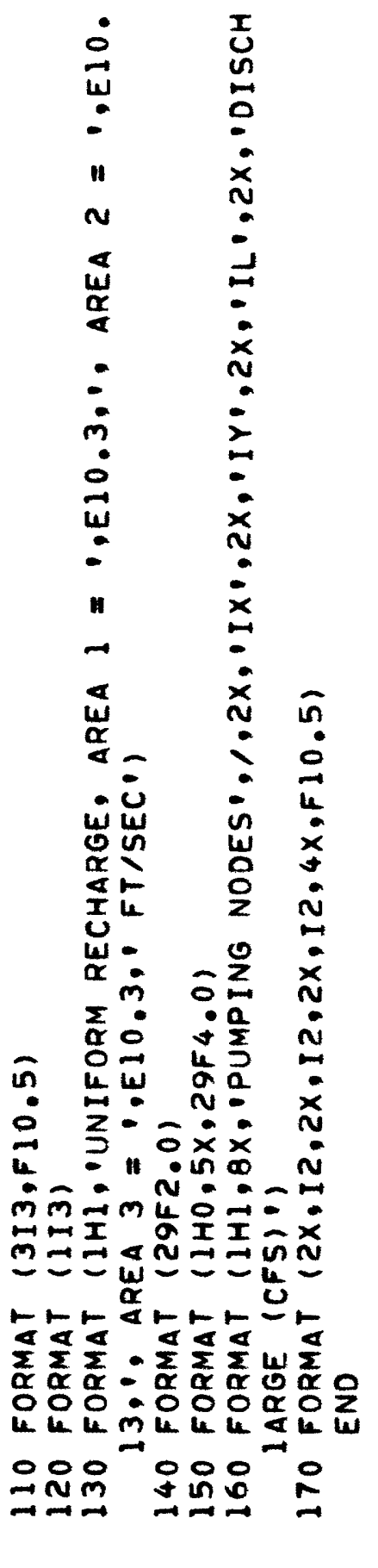


SUBROUTINE ITERAT

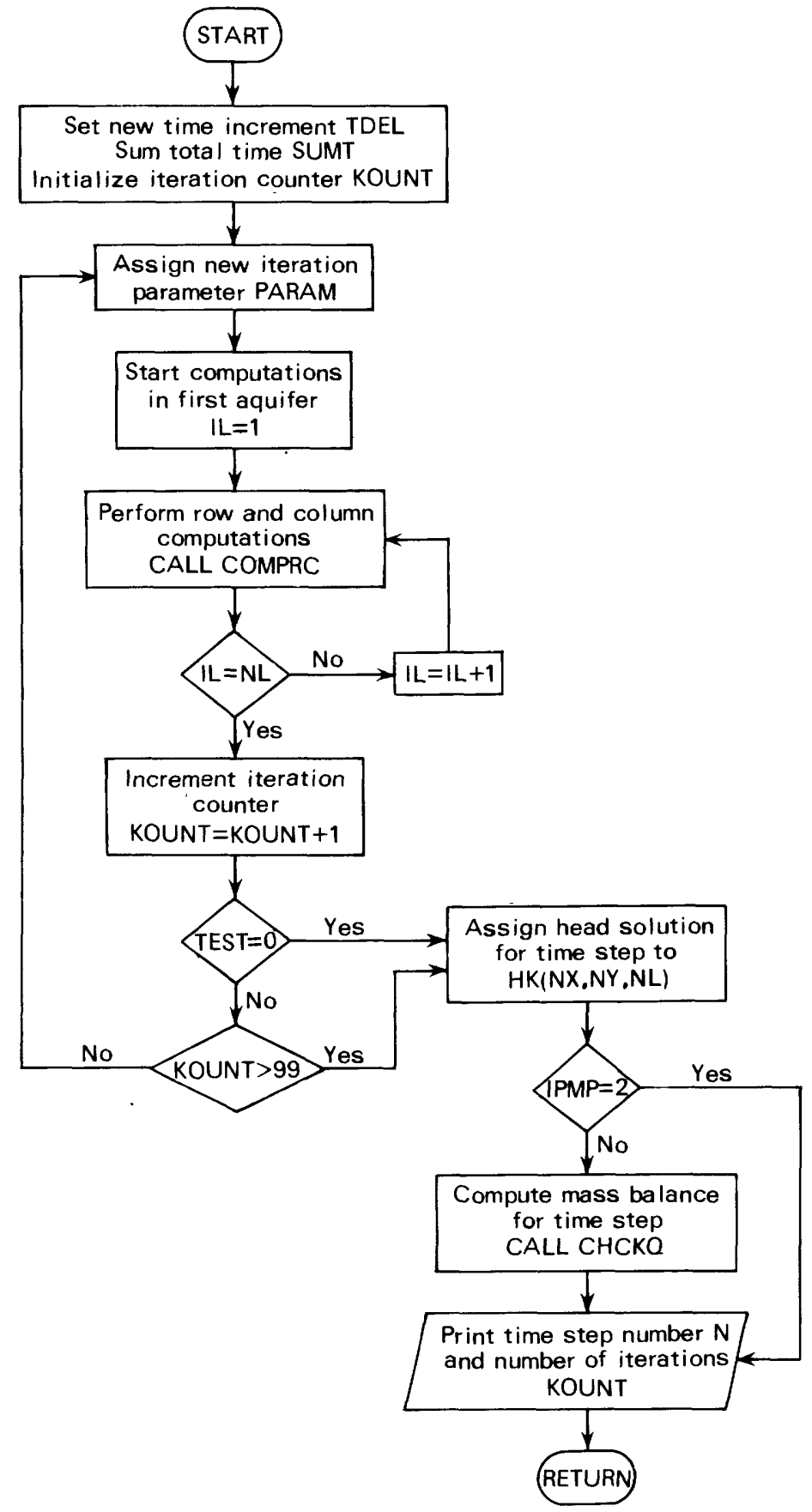


-Nm $\operatorname{tin}$ N

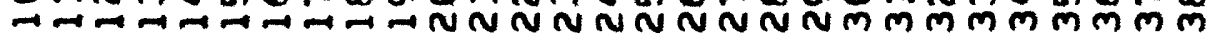

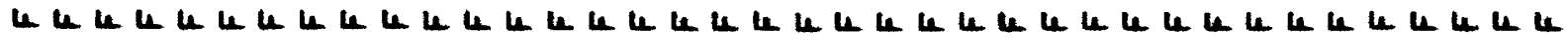

$\mathbf{N}$

$-1$

nmu

प⿺辶

$-\mathrm{N}=$

$z-N$

난

a I $m$

- $-m$

$\sim \hat{\mathrm{N}} \mathrm{a}$

a. $-N$

- $m-$

$-m u$

- L t

a Na

- E -

- a a $5 m$

* o orm

- a $a \frac{a}{a}$

- $z$ z

- $m-2 m$

* $z \pm \neq 0$

* I $I=0$

$+$

$m=-$

- a O - n in

- z

-a

- Nm-

a t-mm

$=-m \times-m$

a muar.

* zar

* $\div \div \frac{1}{2} \div \mathrm{L}$

$-m-a-I>$

- $\operatorname{\omega an}$ a

* zu<- -

- - a a ma

$N * m-m-N$

1. I-0.m-

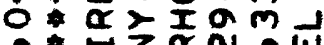

* I $>a+a$

$-a c 0$

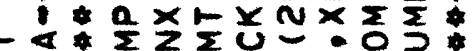

$\Delta=0$ - TU T T

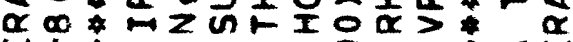
$\omega *$ $-0$

- $u$

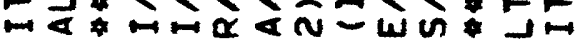

$\omega * \sum \sum \frac{2}{2} 0-\sum \sum \omega * u$

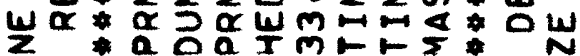

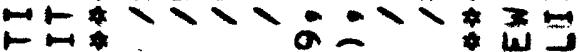
50 z z z zN N z z On $1000-00 \%$

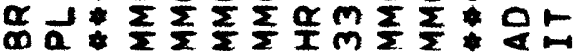
कa

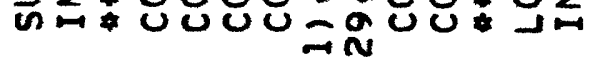

a.

n $?$ a $\frac{1}{2} z 2$

\% OO L

* $\mapsto 0$ * $-0-\alpha$

* $a \geq \leq w$

$\alpha \propto \alpha \geq$

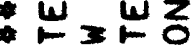

-

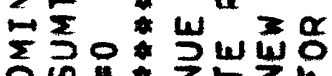

Oे

1 $5=-20$

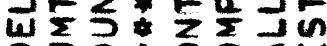

은

$-I$

$z z$

$i-F$

zo z

$\because 00<00$

15⿺辶

90 in 0120

o iv u i wo N $2 \leq z \leq \leq 1$ 인요

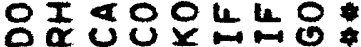

$\operatorname{li}_{-1}=0$

0

$\mathbf{v}$ 


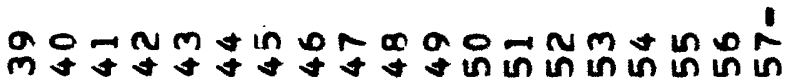

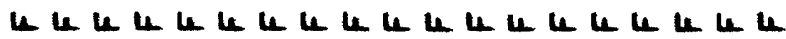

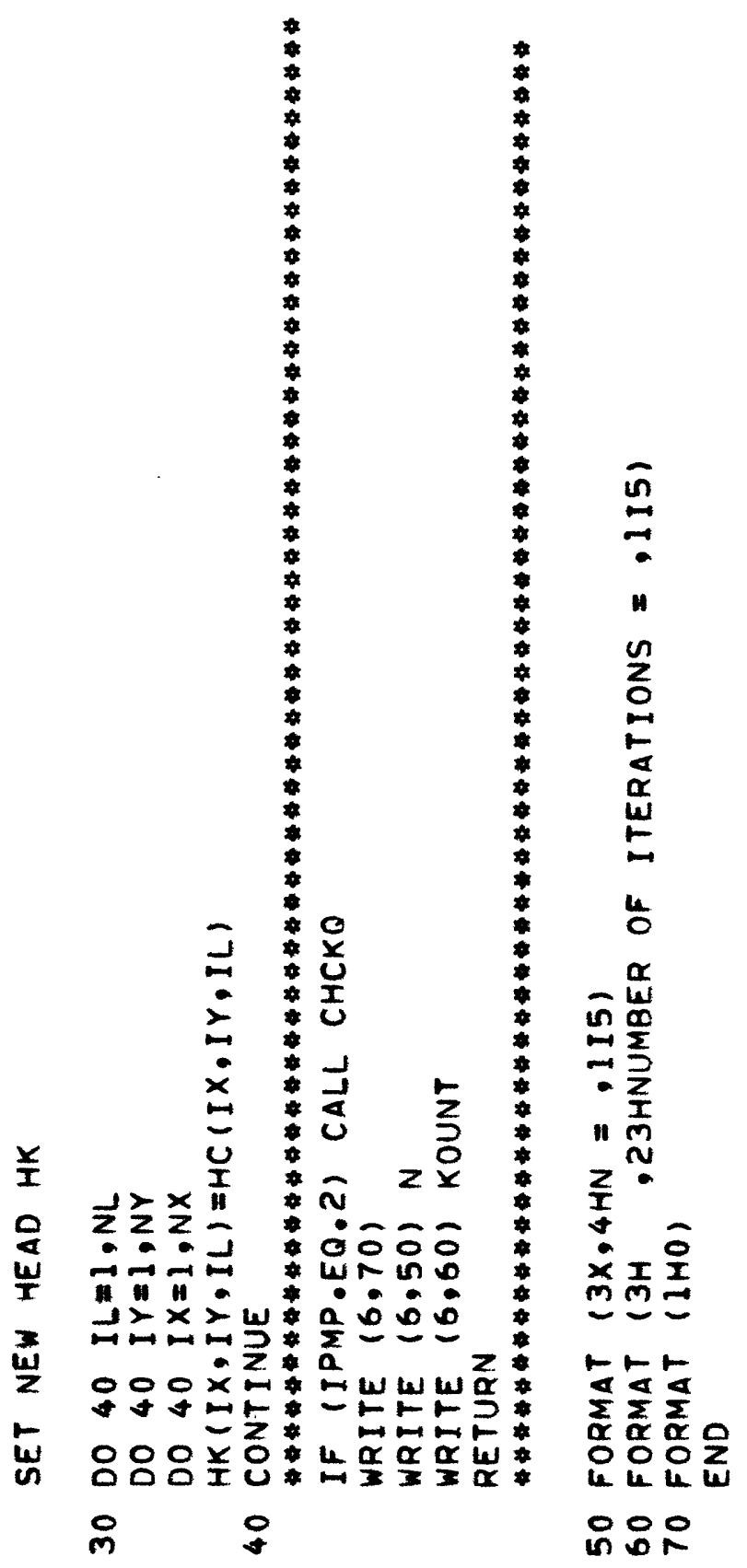

$\boldsymbol{U} \omega \boldsymbol{U} \boldsymbol{U}$ 
SUBROUTINE COMPRC

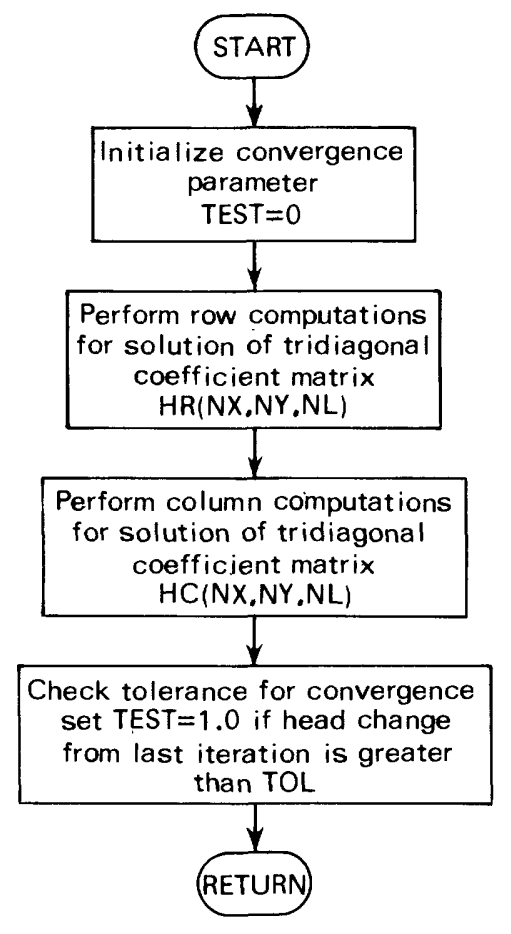




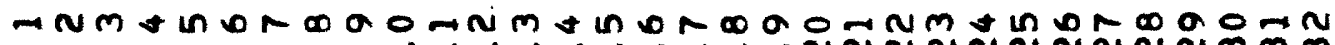

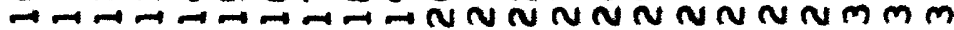

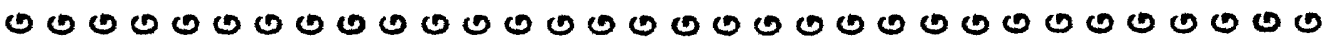

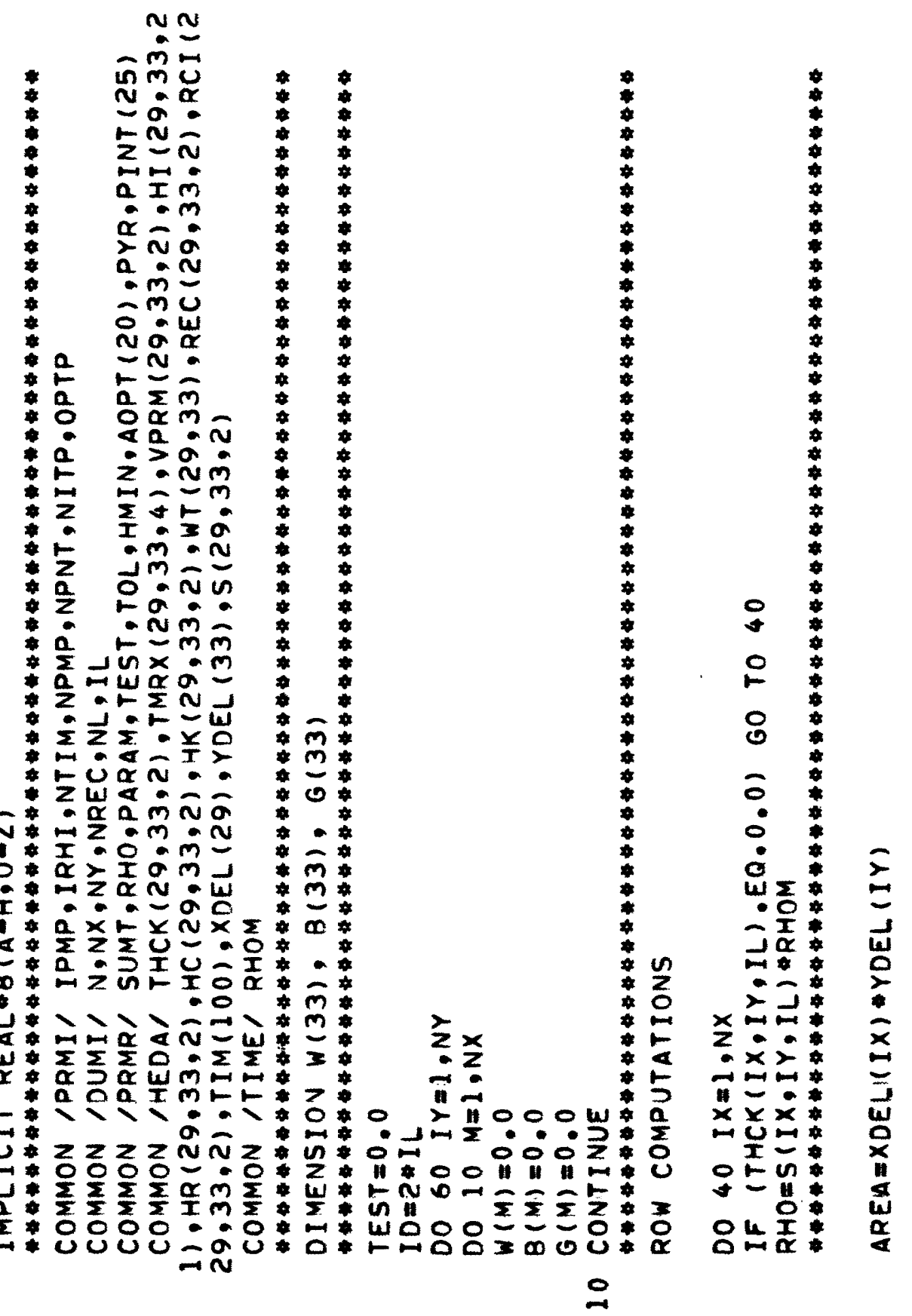
$\boldsymbol{U}$
$\omega \omega$
$\cup \cup \cup$
$\cup U \cup U$ 


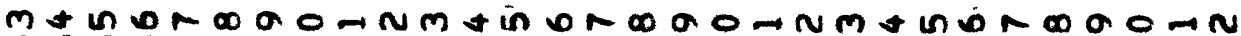

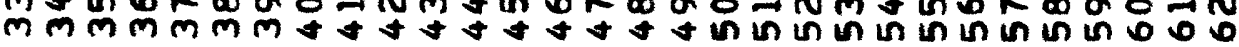

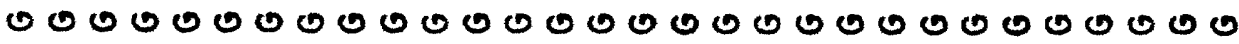

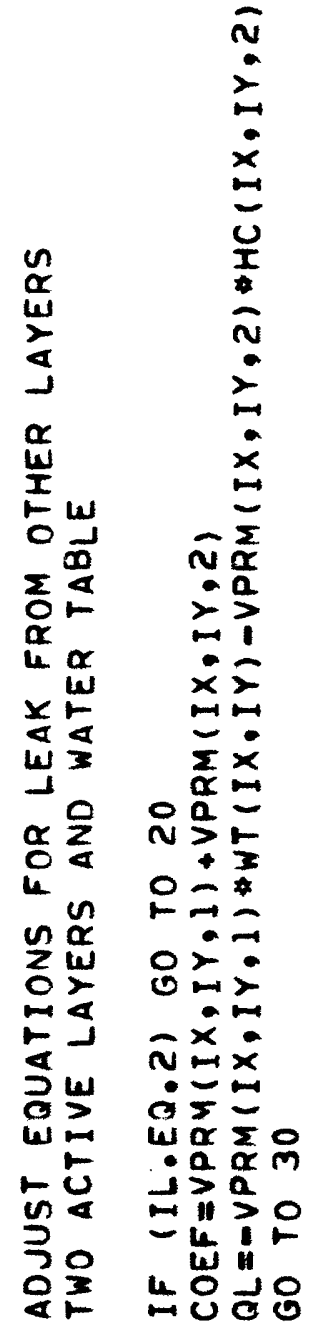

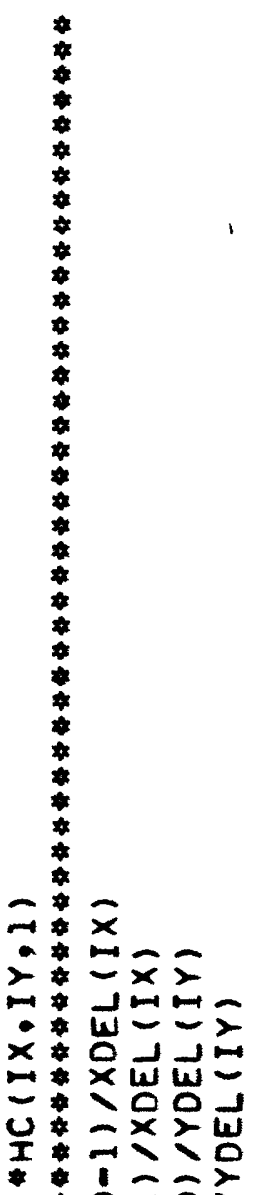

N-10 02

$\rightarrow \infty \rightarrow 0$

$m>>-\infty$

$-i+m-1.20$

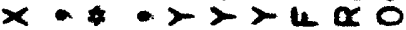

$4 \times *-m+2<x$

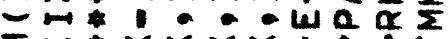

$\Sigma=x \times x \times+4$

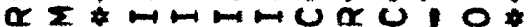

a a $=-2$ $>a+x \times x<a<+a$

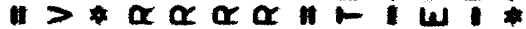

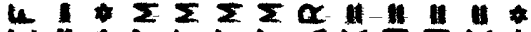

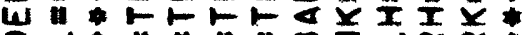
034 in in a c i ja a 列 n

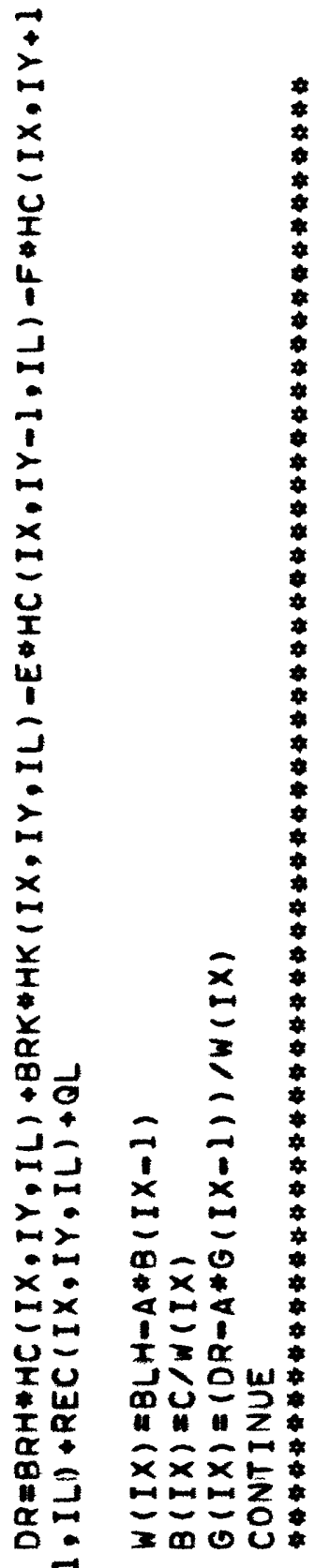

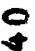

$\omega \omega 0$

$\omega$

3

16

(3)

(3) 


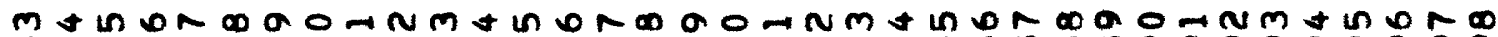

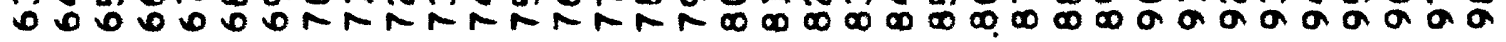

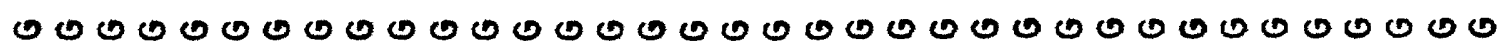

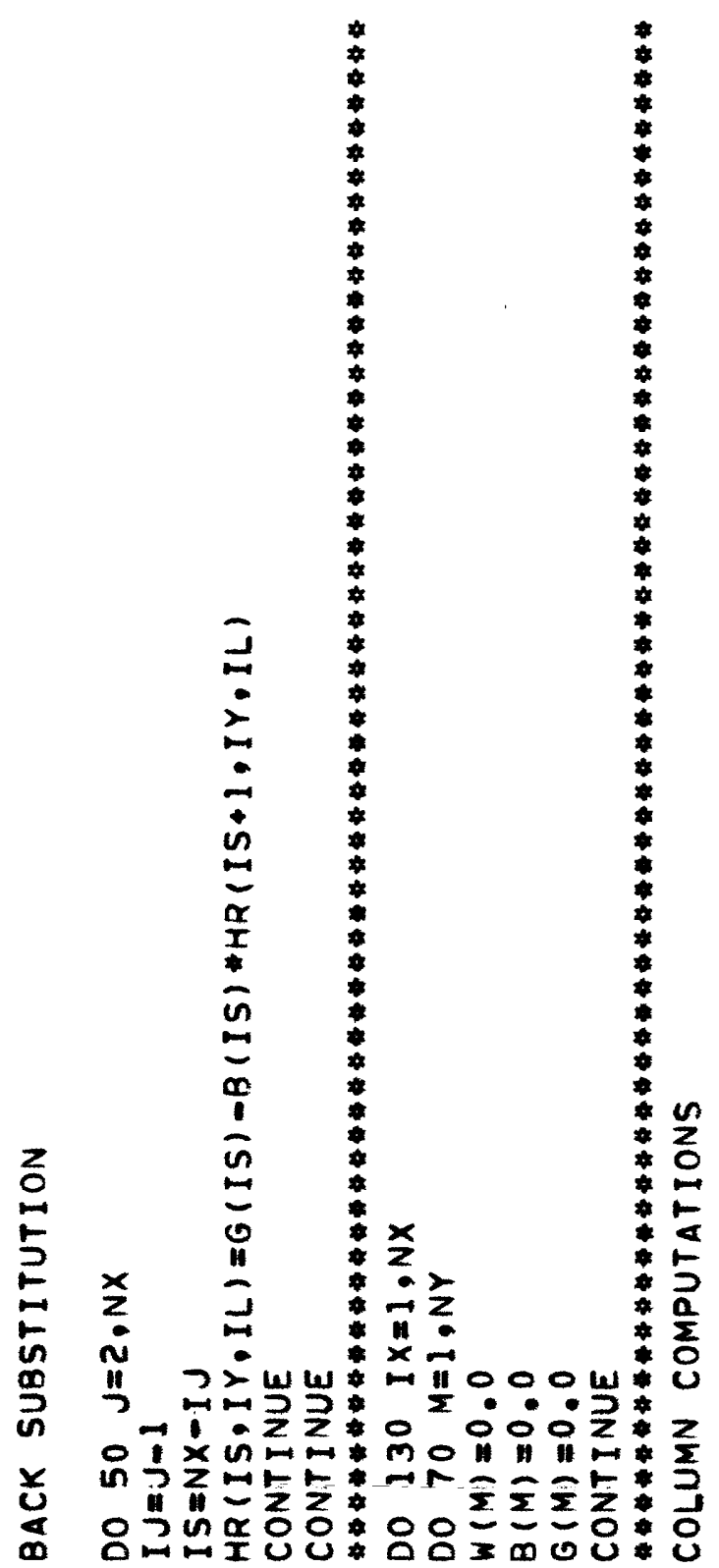

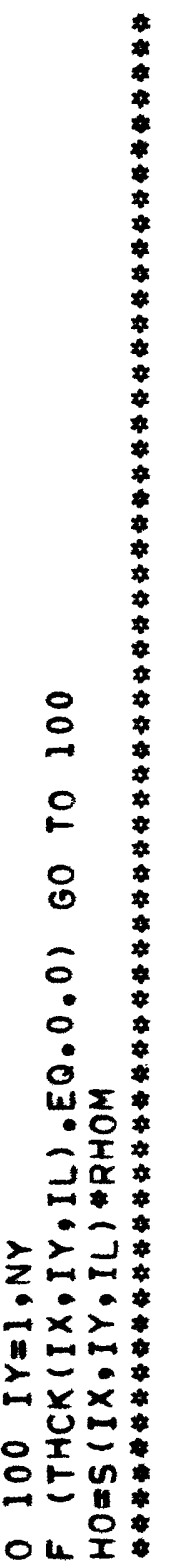

$\lim _{0} \frac{1}{\alpha}$ $\ln _{\infty}$
1

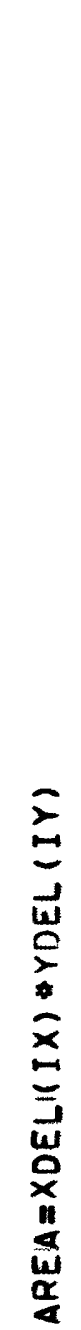

N

$n$

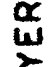

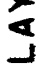

$\alpha$

II

$0 \frac{1}{6}$

$\sum_{0}^{2}$ a

La

$x$

a

w

$=\quad \alpha$ 일

$\frac{\alpha}{6} 2$

L.

in

n $\alpha$

$2 w$

in

$-1$

5 is

o $>$

uा

$-5$

ज4

30

$2=$

o a

$\infty>$

$0 \div$

$-7=$

$8>$

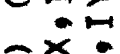

n $m$

- $=$

a $=$

- $\frac{\alpha}{\alpha}$

$1>a$

$-n>0$

wi

$10 \div 0$

岁这

$\infty$

$\frac{2}{\alpha}$

$>a$

$n>$

i.

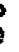

00

$\omega$

$\omega \omega U$

$\cup u$

UUU

$u$

$\omega$ 


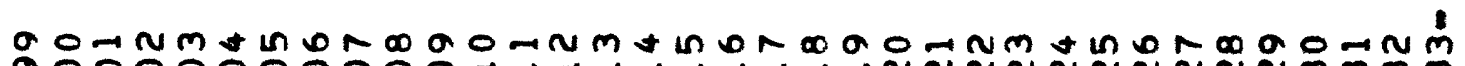

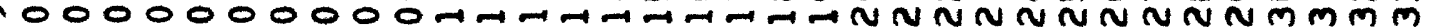

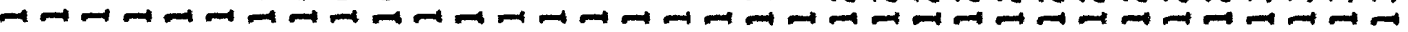
0 0 0 0 0 0 0 0 0 0 0 0 0 0 0 0 0 0 0 0 0 0 0 0 0 0 0 0 0 0 0 0 0 0

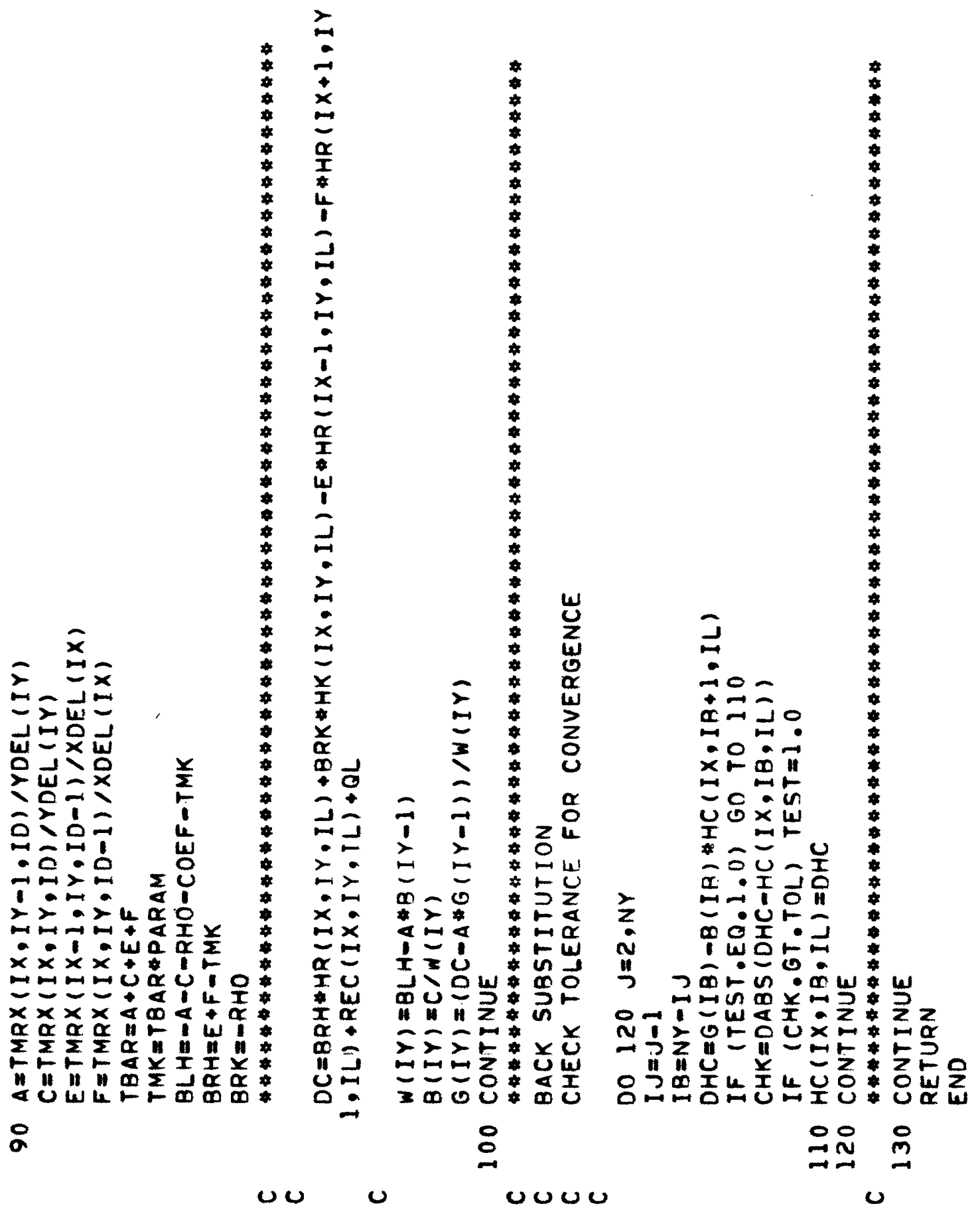




\section{SUBROUTINE OUTPT}

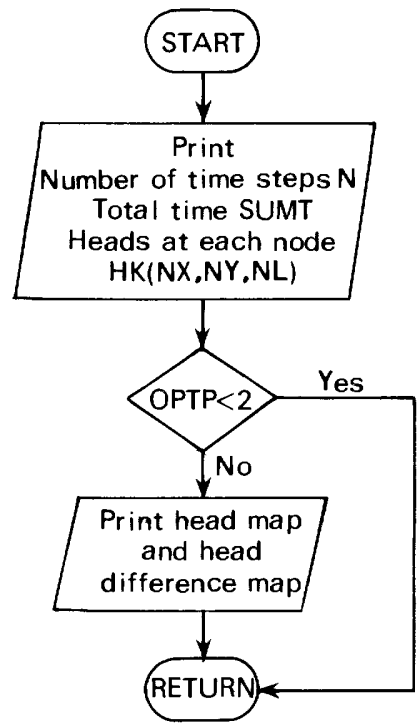




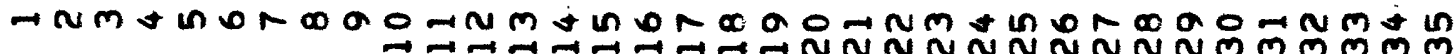

IIIIIIIIIIIIIIIIIIIIIIIIIIIIIIIIIII

N

$\ddot{m}=$

in $m u$

No $\alpha$

$-N-$

$z-n$

H.

a. Im

- $m$

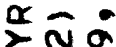

a. $\rightarrow$ N

$m-$

-mu

의

a - n.

*

* o orm

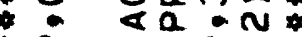

+ a $\quad>a$.

* $z z-N m$

$+m-\infty m$

* Z $\Sigma \leqslant t<$

- I - I a

- $-m \cdot N$.

* Z

* 2 - 0 -

- - Nm-

*a $\quad-1 m m$

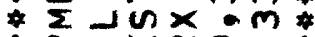

- a w wa 0 -

- Z - ENJ

* $\Sigma \equiv \leq 1-240$

$+4 a-\frac{1}{1}-4$

* $-\omega a n-*$

* zWa-

- a amán

$N * m+m \cdot n *$

$1 * I * 0 \cdot m-*$

$0 * \bar{a}>I a m \omega$

$-4 z a r \cdot \omega *$

$I *-0-00$

- $a x-y N x$

* IZZEU上.

m- $a$ a $D I U-*-4$

a $\infty \sqcap$ Z

$1+\neq$ $-0 * r_{0}+0 \leq$

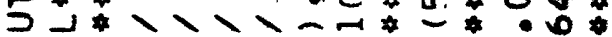

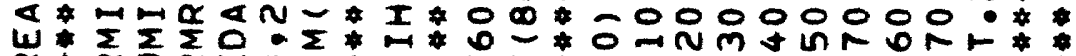

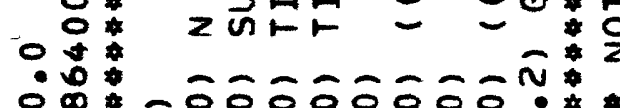

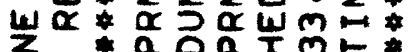

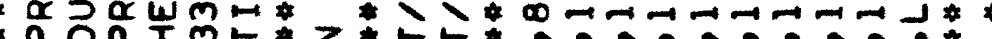

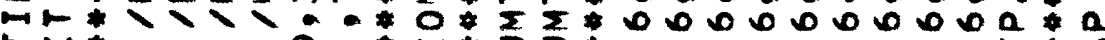

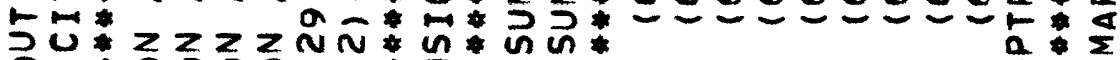

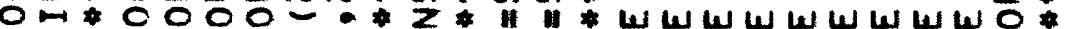

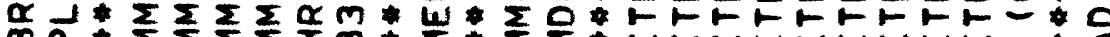

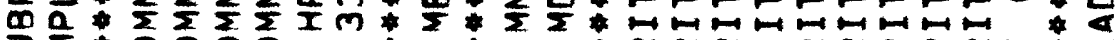

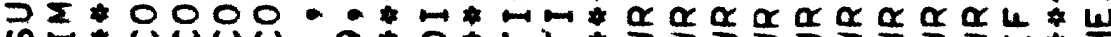

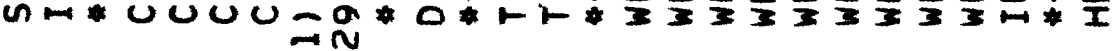

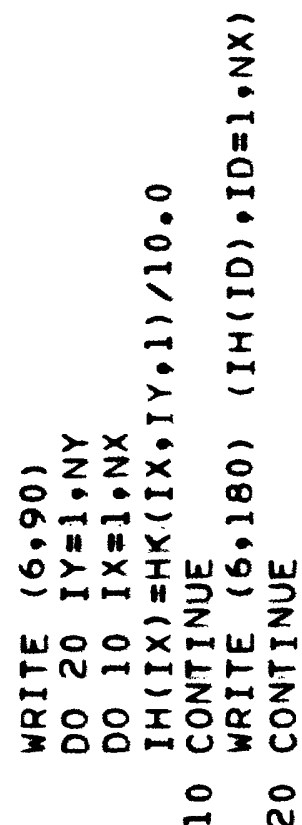

(3)

60

6

000 


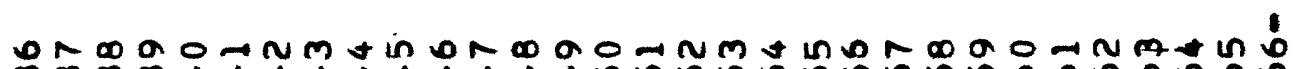
m $m$ mm t IIIIIIIIIIIIIIIIIIIIIIIIIIIIIII

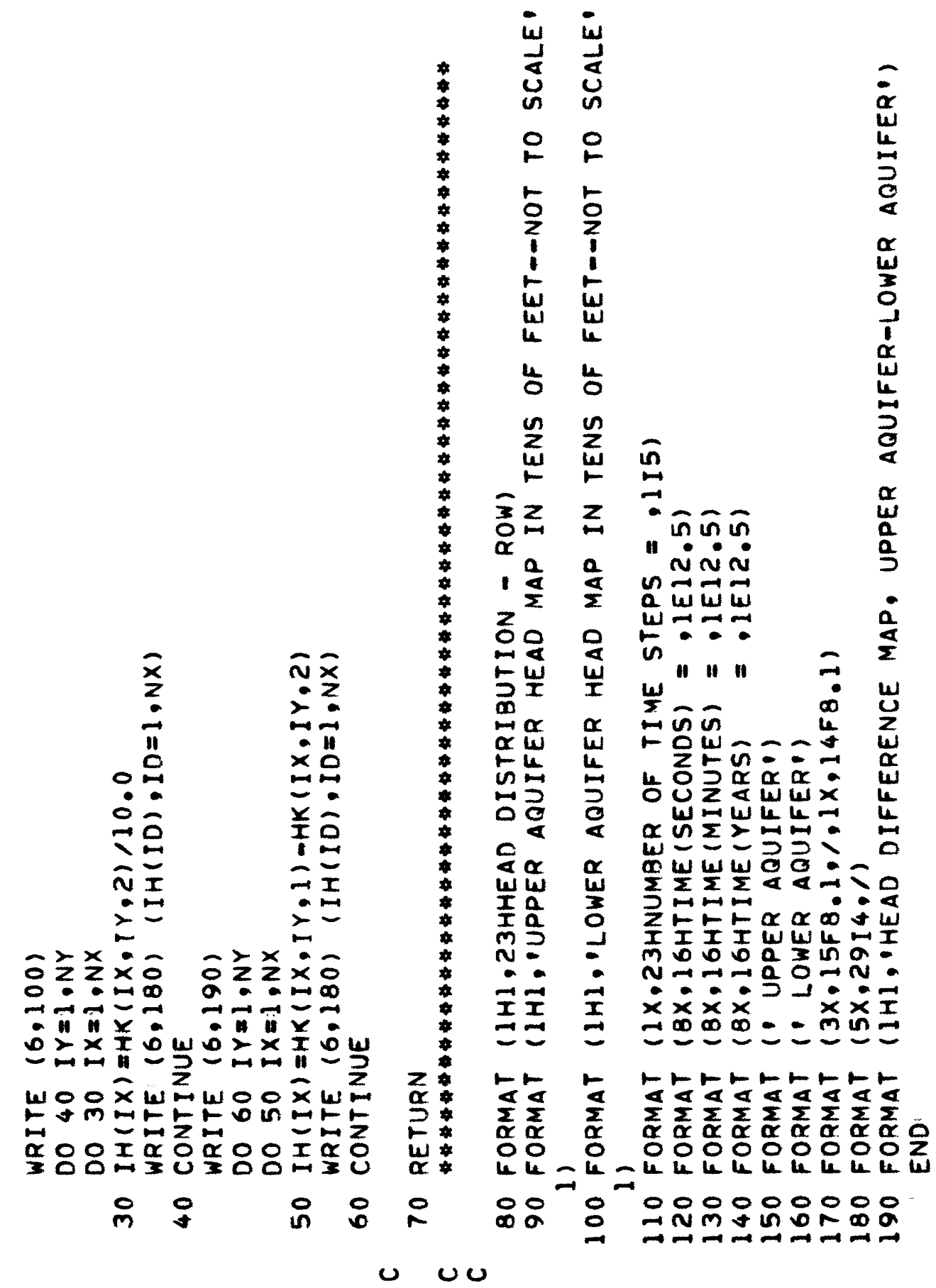




\section{SUBROUTINE CHCKP}

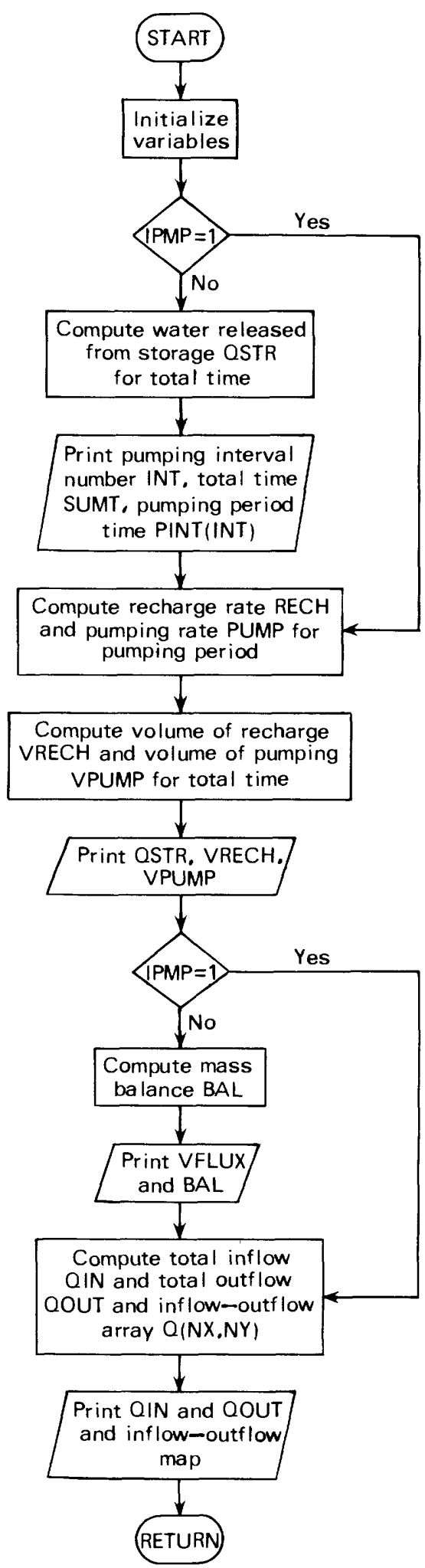




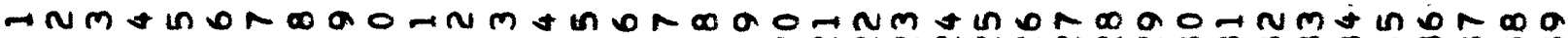

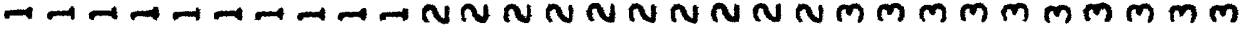

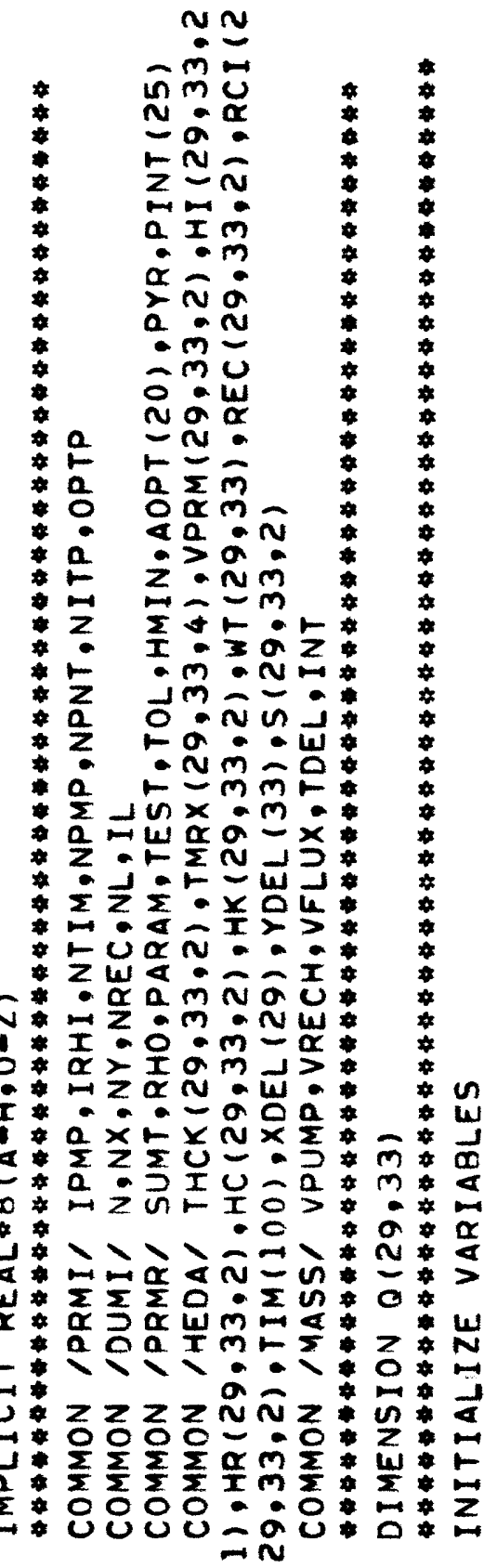

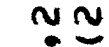

in $m u$

$\underline{a}$

$-\mathrm{N}-$

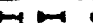

a. Im

$-m$

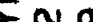

a. $N$

- $m-$

- N .

$0=m$

$0 \alpha m$

$<a-n$

zam

$-2 m$

$-m \cdot N \rightarrow$

गी

a o il

- Nm $n$

$m m+$

m.

$-v a n=\ldots$

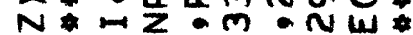

- $I-0 \cdot m-\alpha *$

-

*

$4 * \sum \geq \leq 0-200$

a $* a * D I U-a * m * 4$

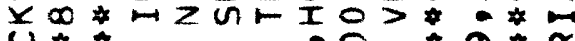

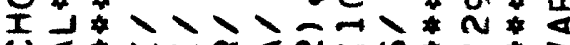

$U<* m a<N-\omega *-*>$

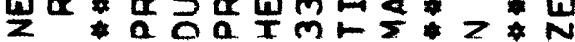

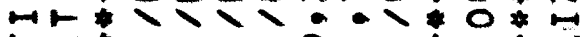

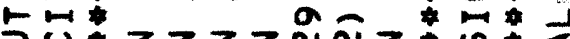

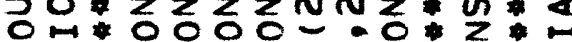

$\alpha\rfloor+\sum \sum \sum \sum \alpha m \sum$ 此

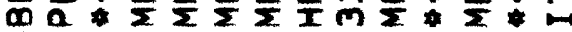

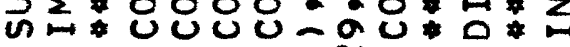
$-N$

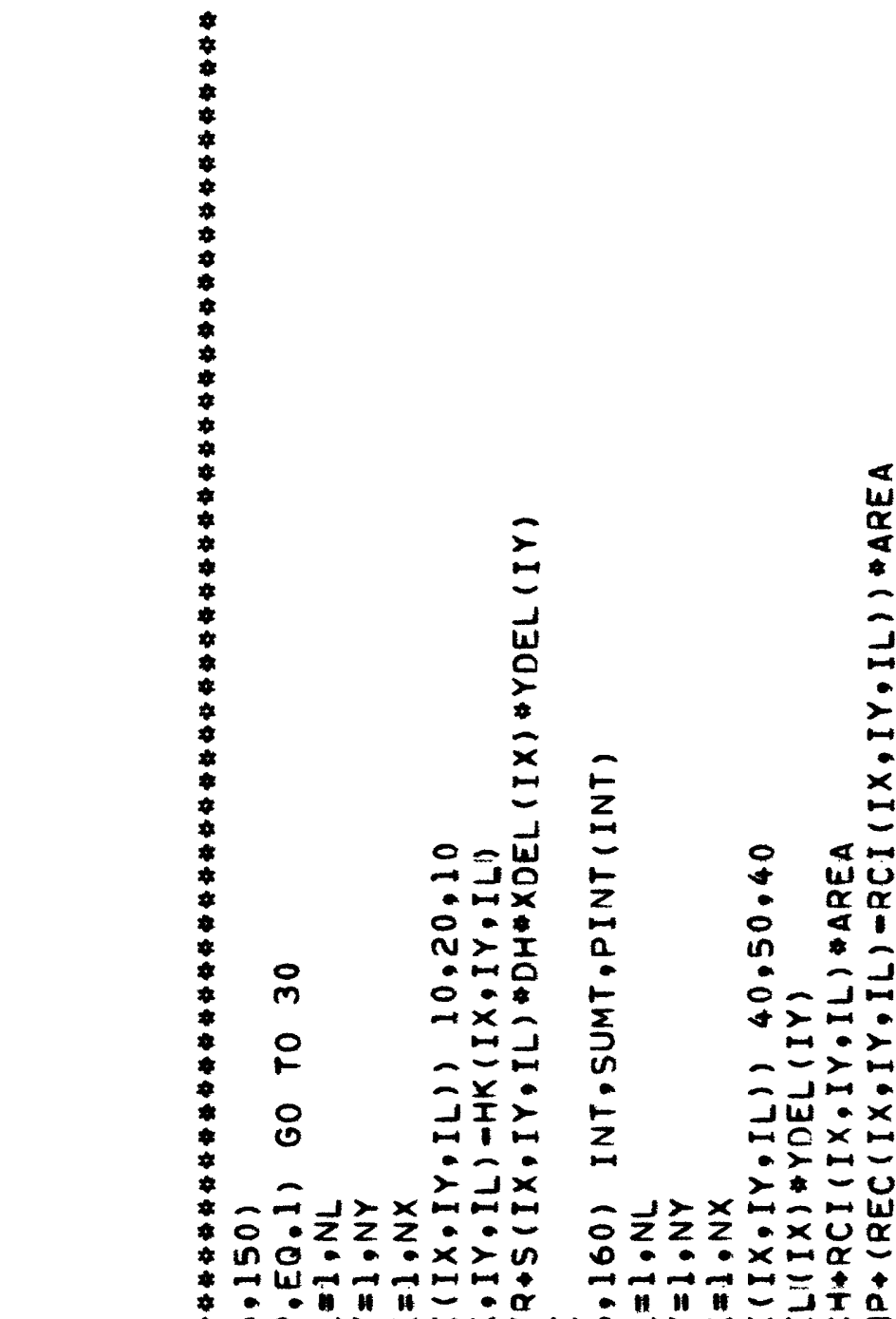

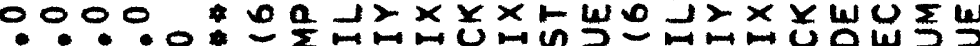

- 4 in -20

- il $1 \mathrm{u}=\mathrm{T}$ 0000 - a I

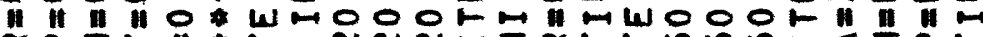

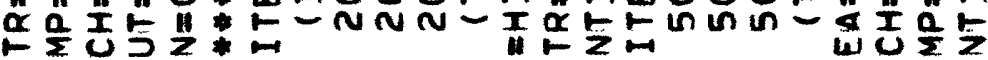

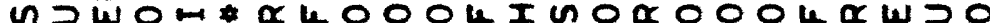

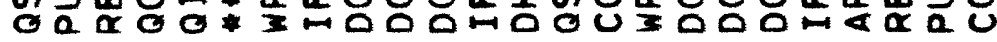

$0 \quad \stackrel{0}{0} \underset{1}{0}$ 


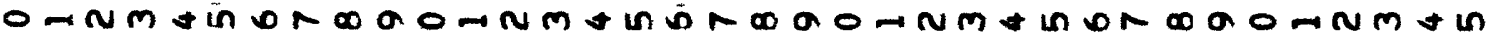

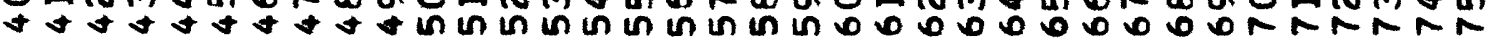

$\underset{\mathbf{O}}{\mathbf{0}}$

$\frac{2}{2} * 0$

施*

* a

* +

* I FEI

* $* 0 \geq z \omega$

a $>\frac{1}{2}$

$>+\infty \infty$

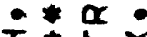

U*

$\omega * 0]$

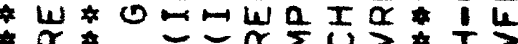

* a *

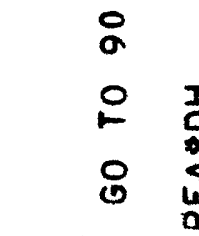

吉 แิ宀 -

$\alpha \rightarrow 0$

$-20 \div 0$

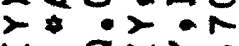

$=0-0>0$

$\sim-w \rightarrow m 0$

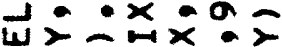

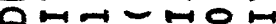

$x \cdot x-\infty$.

* - Izz-a a

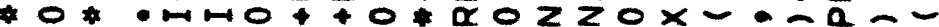

* r a a 0 a I a $>0$ -

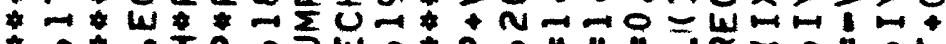

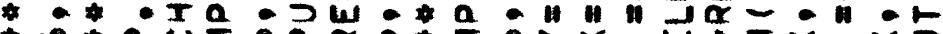

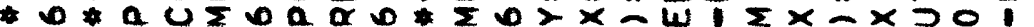

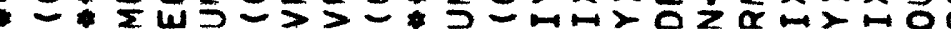
- $\sum_{a} \frac{w}{a}->>-\frac{a}{a}$ 의 $\sum_{a}^{\alpha}=2 \geq 0$

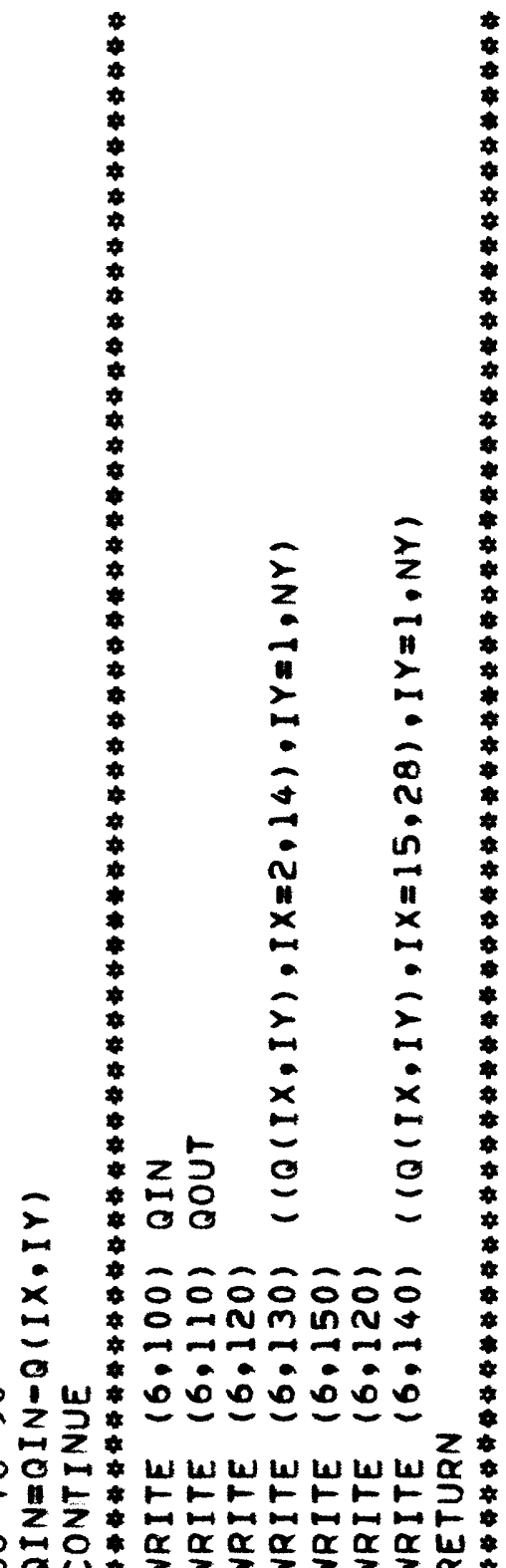

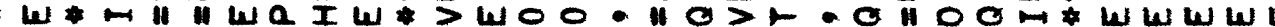
- - - Iat * $\alpha$ U

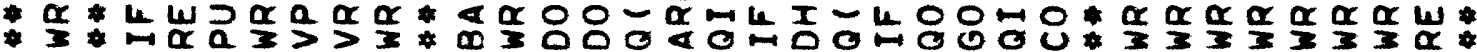

웅 $\therefore \quad 0 \%$ 


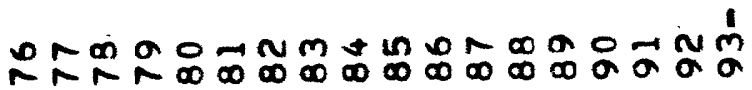

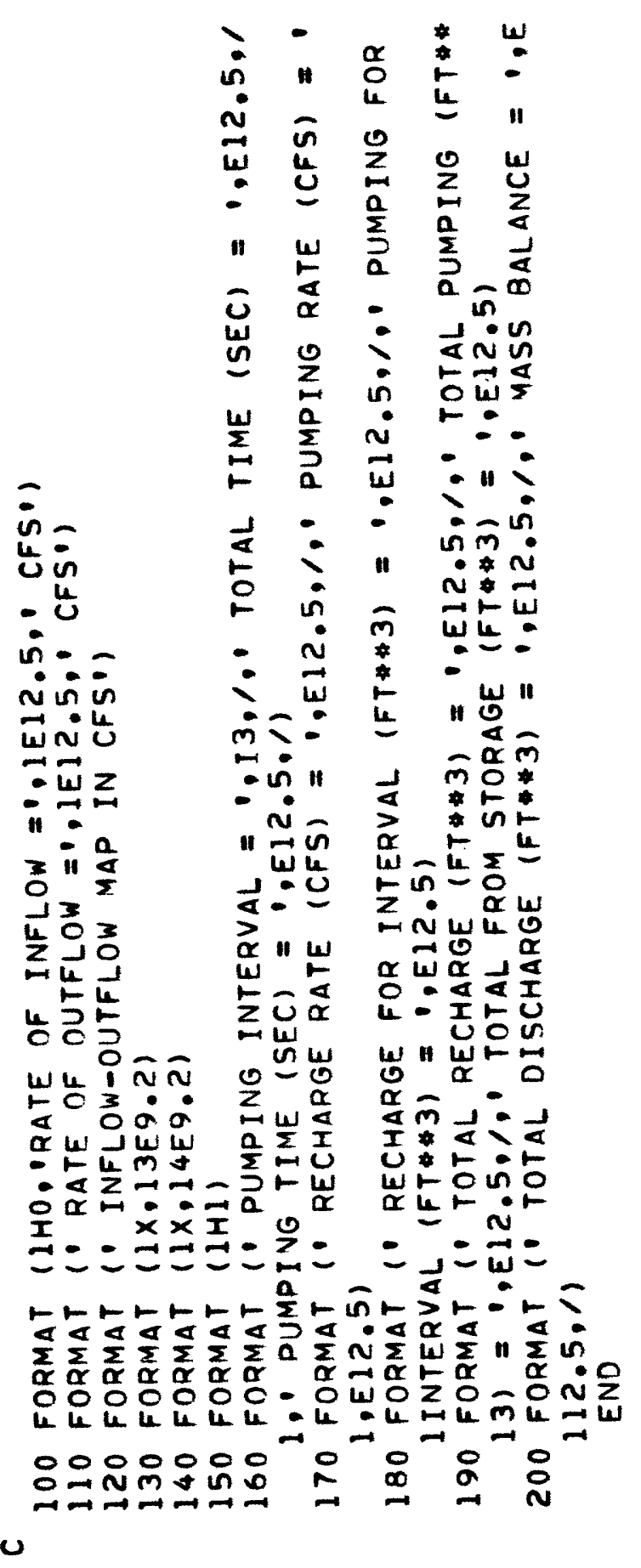


SUBROUTINE CHCKO

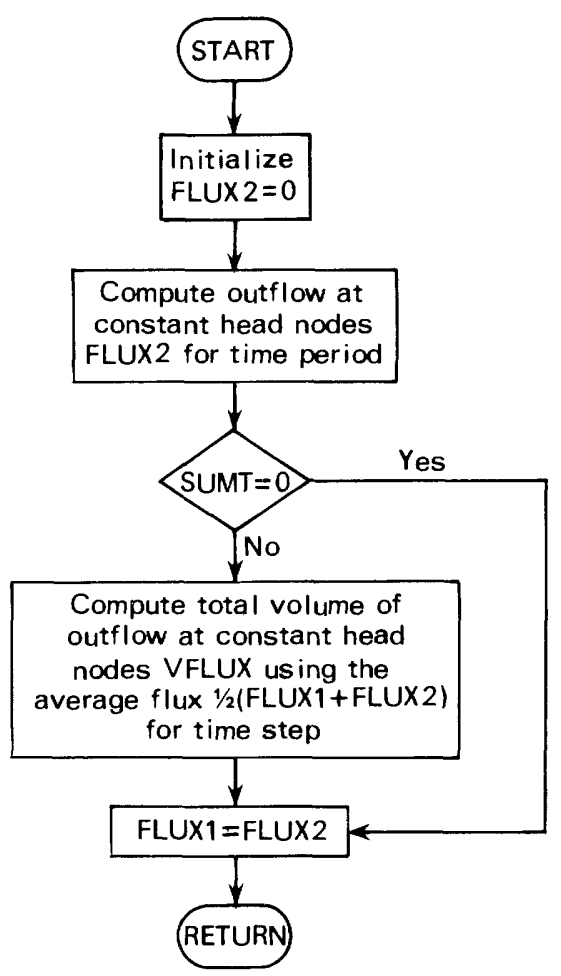




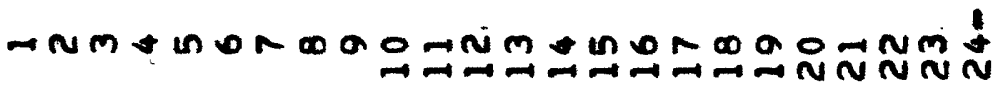

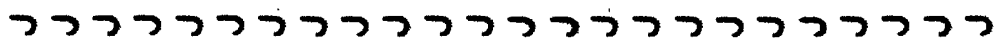

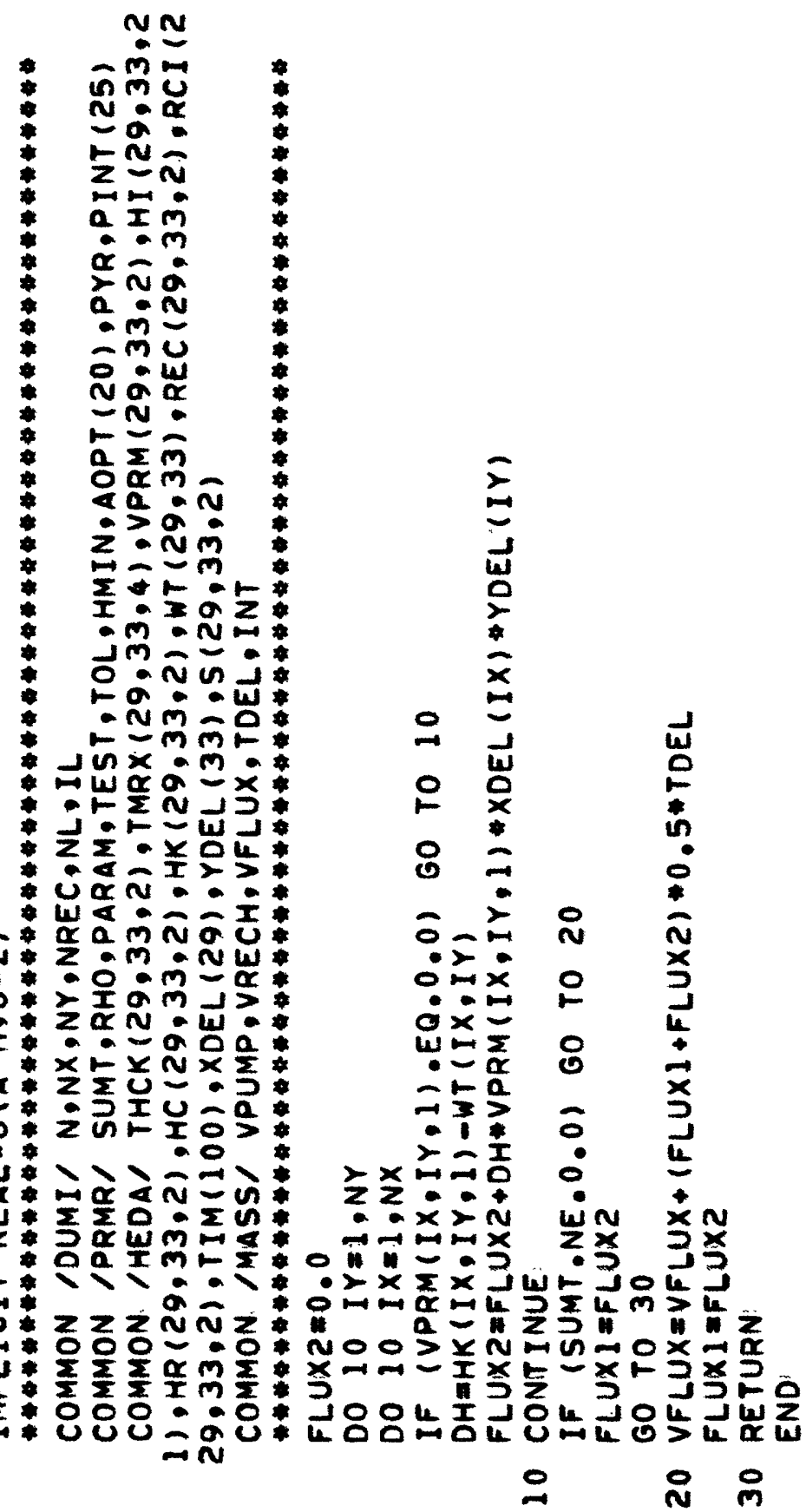

$\boldsymbol{u}$ 
INPUT DATA LISTING

\begin{tabular}{|c|c|c|}
\hline Card numbers & Data set for & FORMAT \\
\hline $\begin{array}{l}1 \\
2 \\
3 \\
4-36\end{array}$ & $\begin{array}{l}\operatorname{PINT}(25) \\
\operatorname{XDEL}(29) \\
\operatorname{YDEL}(33) \\
\operatorname{VPRM}(29,22,1)\end{array}$ & $\begin{array}{l}25 \mathrm{~F} 3.0 \\
29 \mathrm{~F} 2.0 \\
33 \mathrm{~F} .20 \\
29 \mathrm{~F} 2.0\end{array}$ \\
\hline $\begin{array}{c}37-69 \\
70-102 \\
103-135 \\
136-234\end{array}$ & $\begin{array}{l}\operatorname{VPRM}(29,33,2) \\
\operatorname{VPRM}(29,33,1) \\
\operatorname{VPRM}(29,33,2) \\
\operatorname{WT}(29,33)\end{array}$ & $\begin{array}{c}29 \mathrm{~F} 2.0 \\
29 \mathrm{~F} 2.0 \\
29 \mathrm{~F} 2.0 \\
10 \mathrm{~F} 8.2 / 10 \mathrm{~F} 8.2 / 9 \mathrm{~F} 8.2\end{array}$ \\
\hline $\begin{array}{l}235-330 \\
331-426 \\
427-459 \\
460\end{array}$ & $\begin{array}{l}\operatorname{HI}(29,33,1) \\
\operatorname{HI}(29,33,2) \\
\operatorname{RCI}(29,33,1) \\
\text { NREC }\end{array}$ & $\begin{array}{r}10 \mathrm{~F} 8.2 \\
10 \mathrm{~F} 8.2 \\
29 \mathrm{~F} 2.0 \\
113\end{array}$ \\
\hline
\end{tabular}




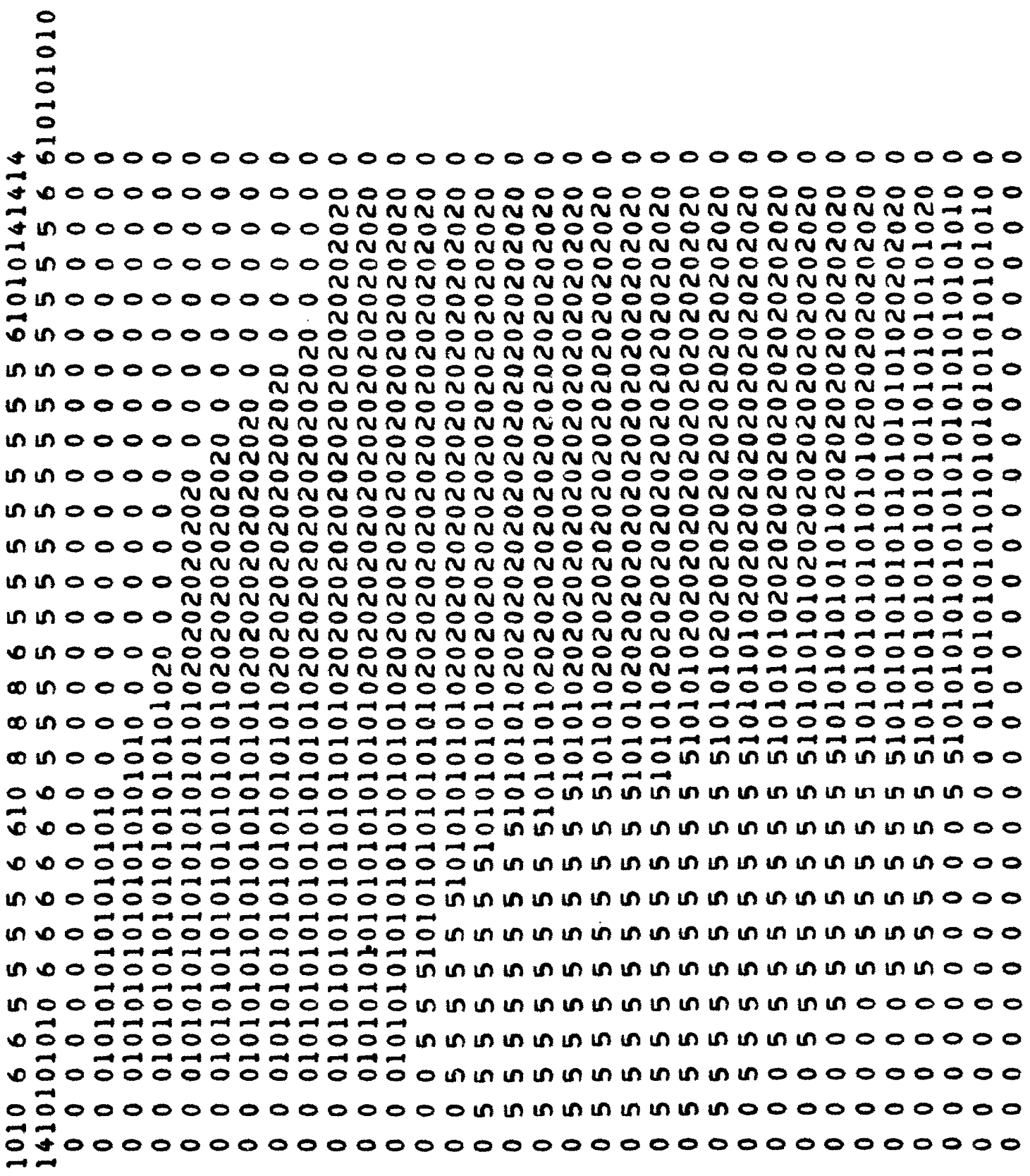

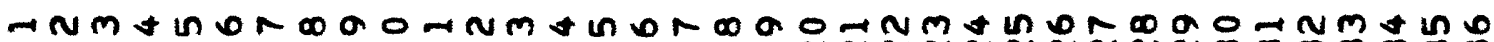

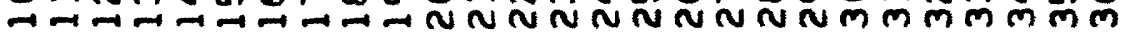

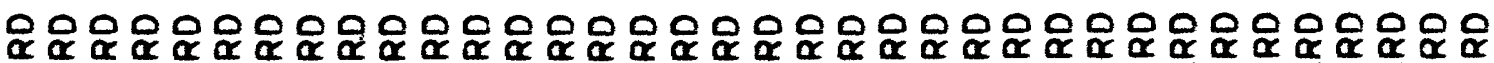

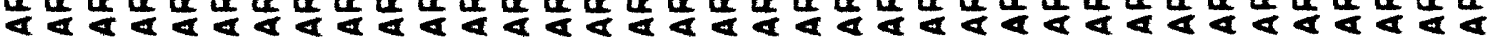
ปٔ 
000000000000000000000000000000000 000000000000000000000000000000000

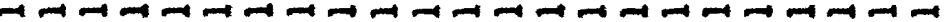
000000000000000000000000000000000

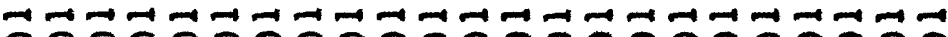
000000000000000000000000000000000

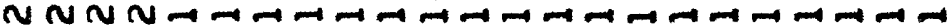

000000000000000000000000000000000

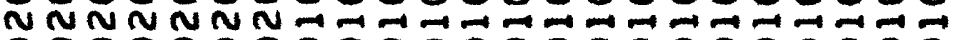
000000000000000000000000000000000

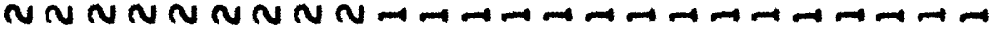
000000000000000000000000000000000

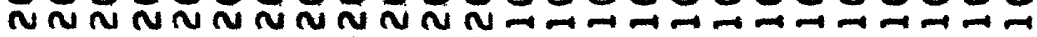
000000000000000000000000000000000

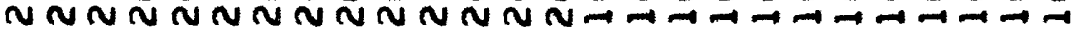

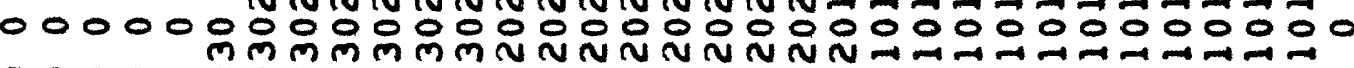

000000000000000000000000000000000

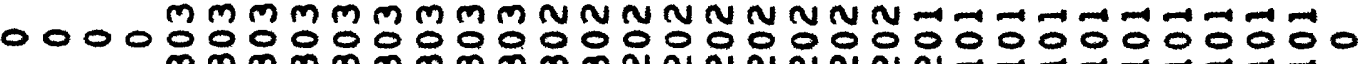

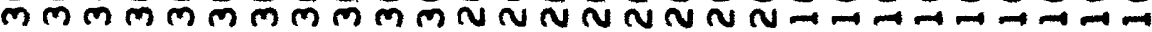

000000000000000000000000000000000

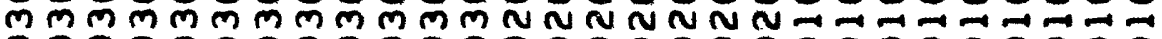

000000000000000000000000000000000

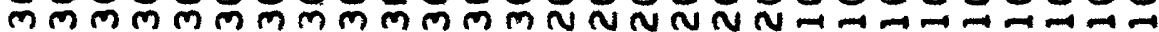

- 00000000000000000000000000000000 $\forall t+ \pm m m m m m m m m m m m$ m

000000000000000000000000000000000

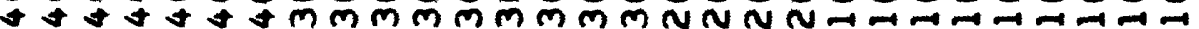

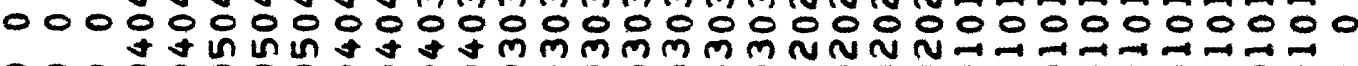

000000000000000000000000000000000

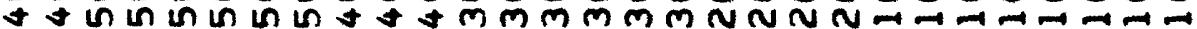

- 00000000000000000000000000000000

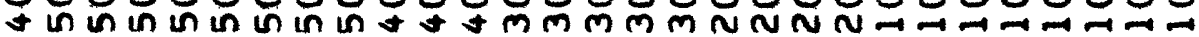

000000000000000000000000000000000

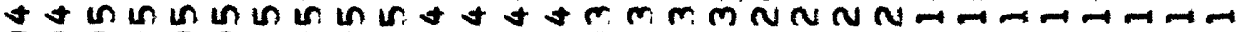

000000000000000000000000000000000

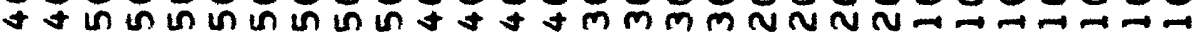

000000000000000000000000000000000

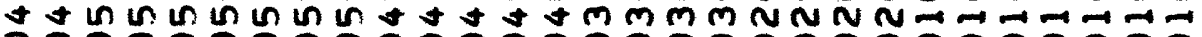

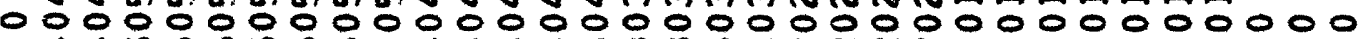

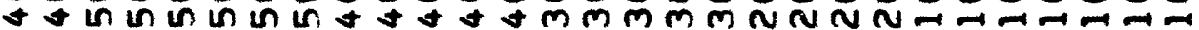

000000000000000000000000000000000

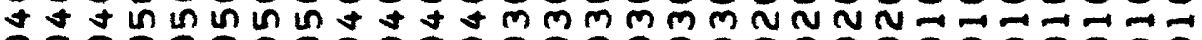

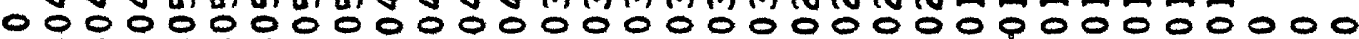

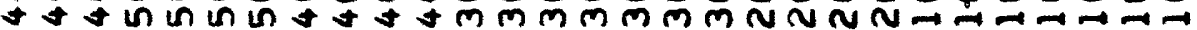

000000000000000000000000000000000 $4 t+t$ in $t+4 t+m m m m m m m n N N N=-1$

000000000000000000000000000000000

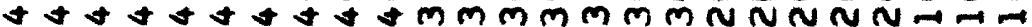

000000000000000000000000000000000 mmm N N N N N

000000000000000000000000000000000

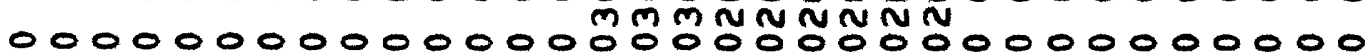

-

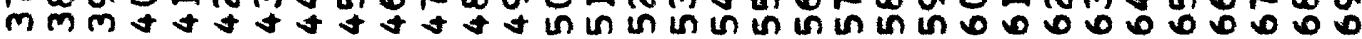

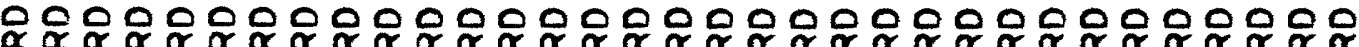

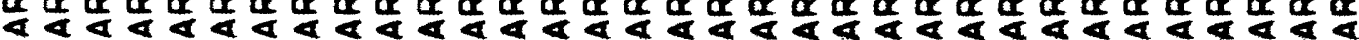

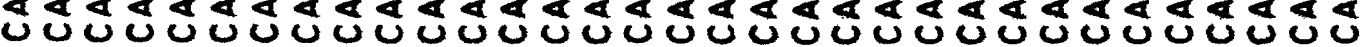


000000000000000000000000000000000 000000000000000000000000000070000 000000000000000000000000000070000 000000000000000070000000700000000 $00000000000007 \overrightarrow{1} 10000000 \% 00000000$ 000000000007700000000007000000000 $0000000000-10$ d́000000000-10000000000 $0000000000 \frac{1}{100000000000 \uparrow 10000000000}$ $000000000 \div 00000000000 \div 00000000000$ $000000000 \frac{1}{1} 00000000000 \frac{1}{1} 00000000000$ $0000777 T_{1}+17000000007000000000000$ $00000000700710000000 \frac{1}{1} 000000000000$

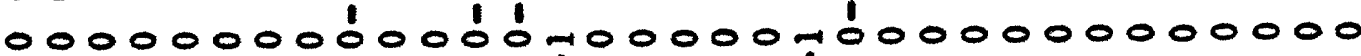

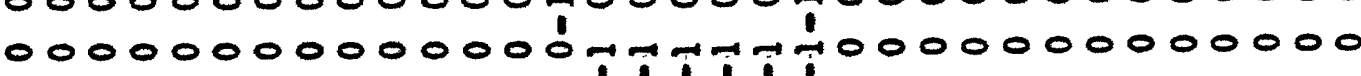

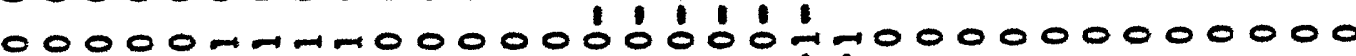

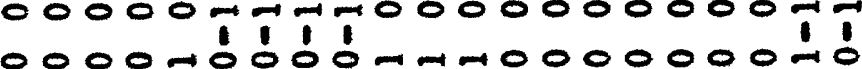

000070000717000000070000000000000

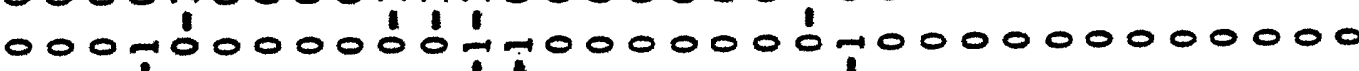

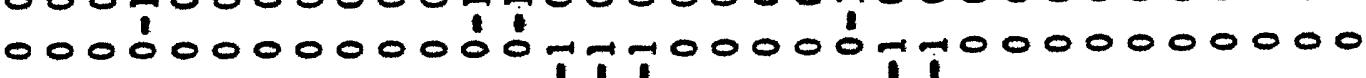

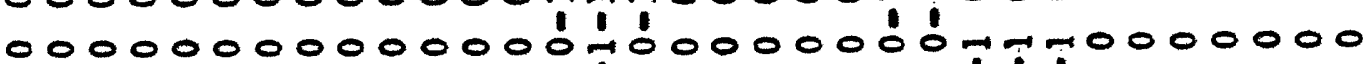

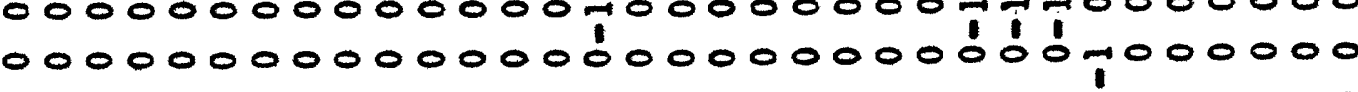

000000000000000000000000000000000 000000000000000000000000000000000 000000000000000000000000000000000 000000000000000000000000000000000 000000000000000000000000000000000 000000000000000000000000000000000 000000000000000000000000000000000 000000000000000000000000000000000

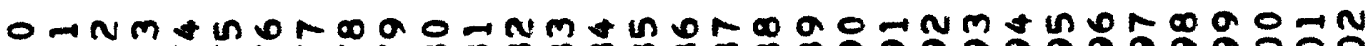

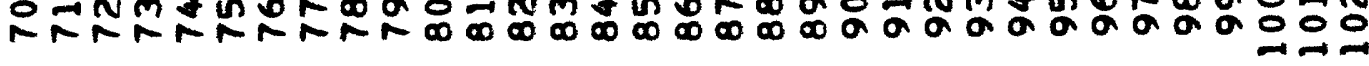

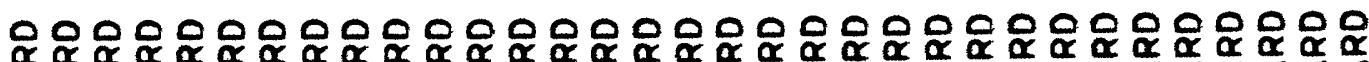

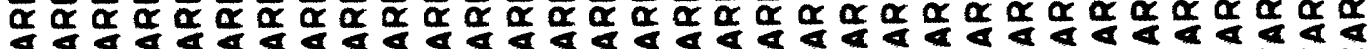

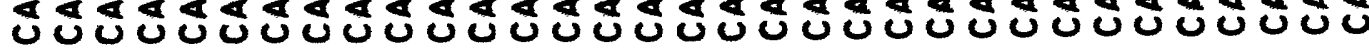


000000000000000000000000000000000

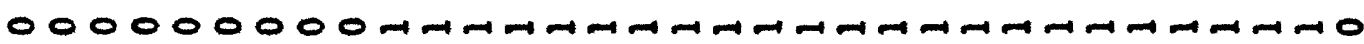

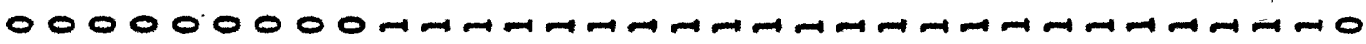

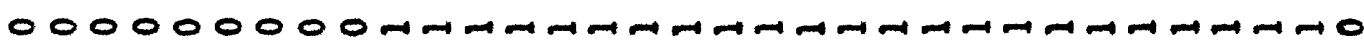

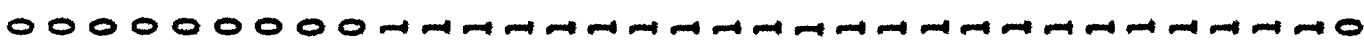

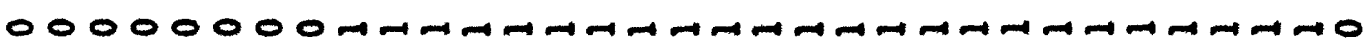

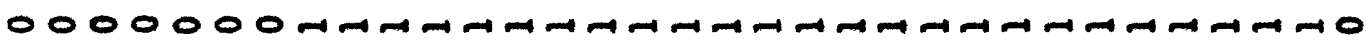

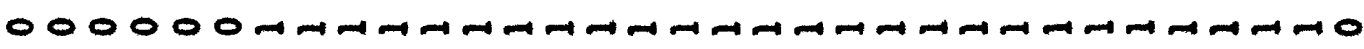
0 0000 o 0 -

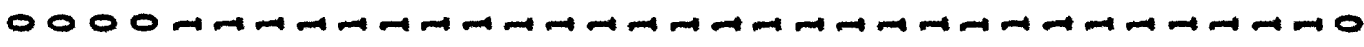

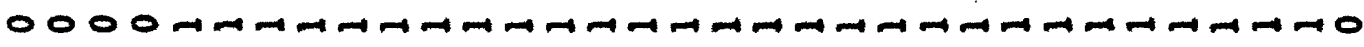

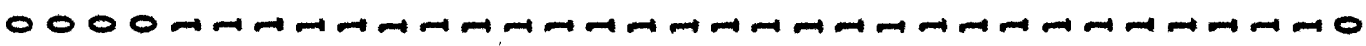

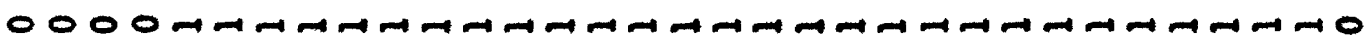

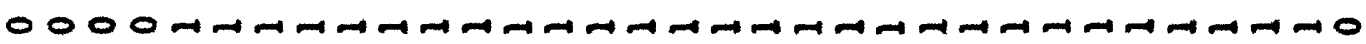
0 0 0 0 00 ○ 00 0 0 0 0 -

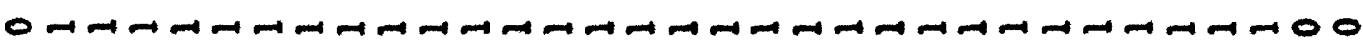

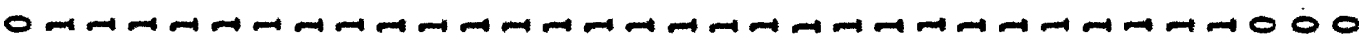

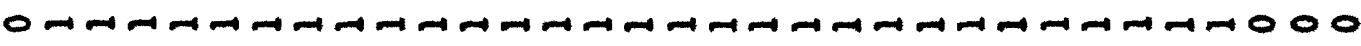

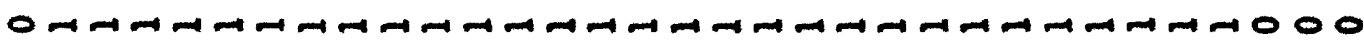
O-

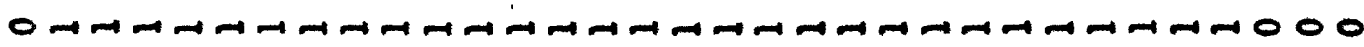

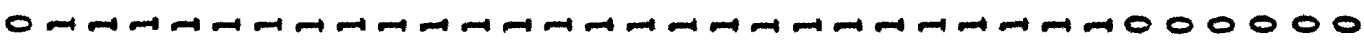
O $00000000000000 m-m-m-m-1000000000$ $00000000000000 m-m m-10000000000$ 000000000000000000000000000000000

m\$n

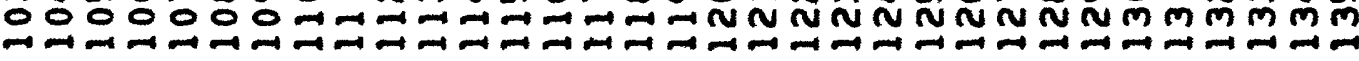

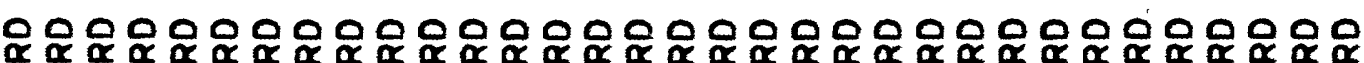

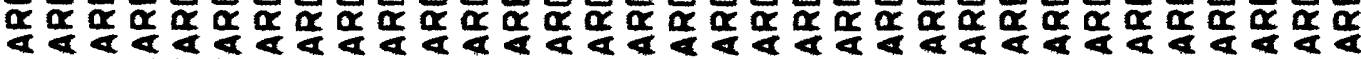

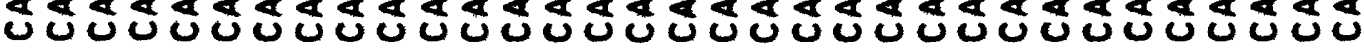




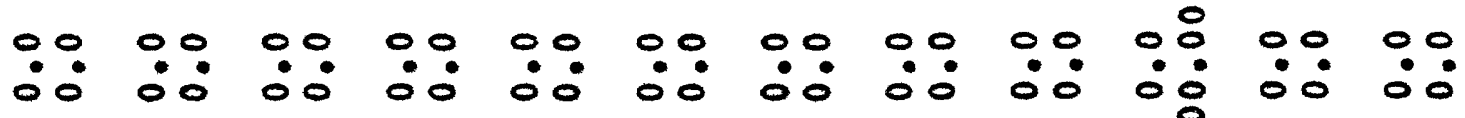

000000000000000000000000000000000000 ட் 贻

000000000000080000080080000080000000

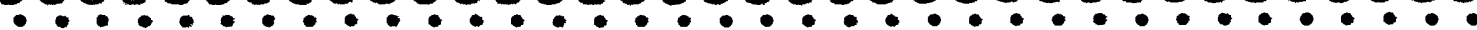
$0000000000000 \ln 00000000000000000 \ln 0000$ in in in in in

000000000000000000000000000000000000 $\therefore \circ 0 \circ 000000000000000000000000000000$ i 車

000000000000000000000000000000000000

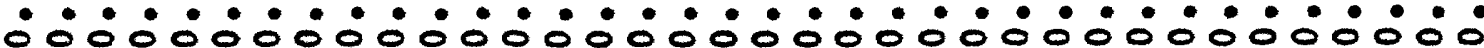

000000000000000000000000000000000000

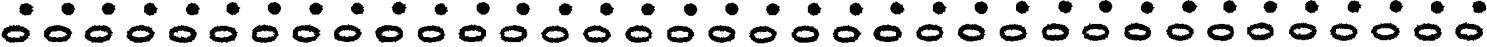

000000000000000000080080000000000008

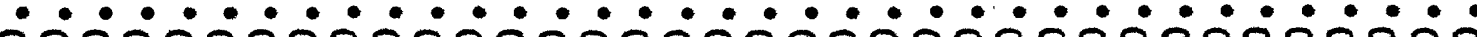
000000000000000000000000000000000000

000000000000080000000000000000000000

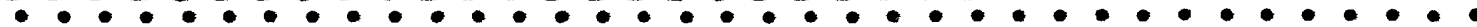
000000000000000000000000000000000000

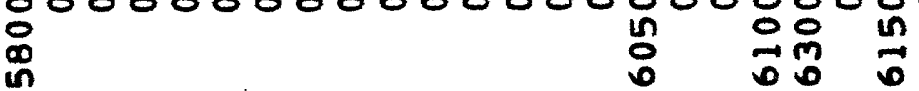

000000000000000000000000000000008000

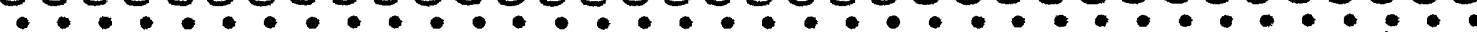
000000000000000000000000000000000000 $\stackrel{0}{1}$

in

N $\mathbf{N}$

000000000000000000000000000008000000

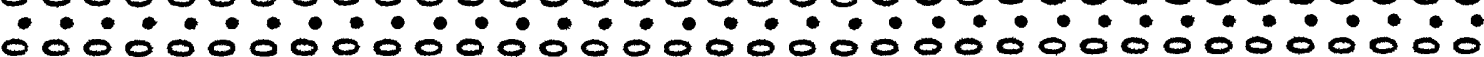
0 $\overline{0}$

ON

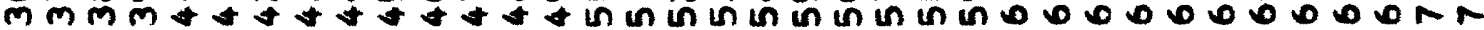

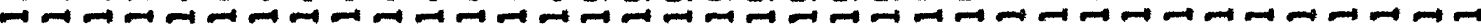

응ㅇㅇㅇㅇㅇㅇㅇㅇㅇㅇㅇㅇㅇㅇㅇㅇㅇㅇㅇㅇㅇㅇㅇㅇㅇㅇㅇㅇㅇㅇㅇㅇㅇㅇㅇㅇㅇㅇㅇㅇㅇㅇㅇㅇㅇㅇㅇㅇㅇㅇㅇ웅 


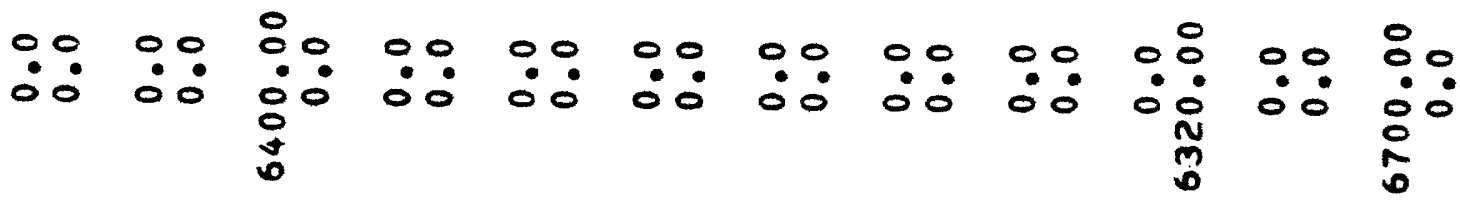

000000000000000000000000000000000000

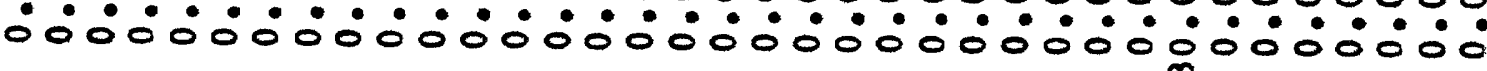
N

000000000000000000000000000000000000 ட் 莎

ฟิ

000000000000000000000000000000000000

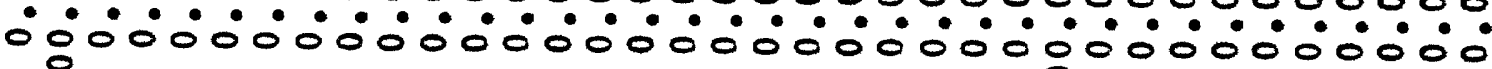
8 N

000080000000008000000080000000000000

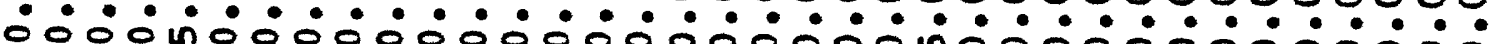
N $00000000000000000 \frac{1}{N}$
0

0000000800800800000000000000000000000

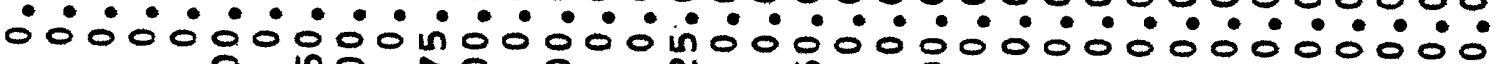

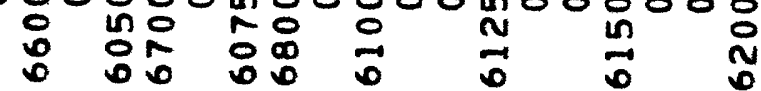

000000000000000000000000000000000000

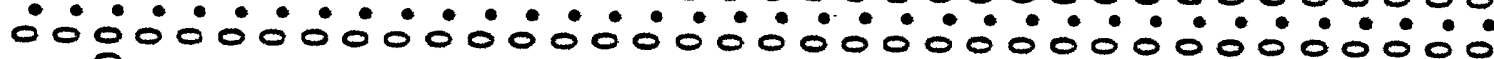
in

○

ñ

6

000000000000000000000000000000000000

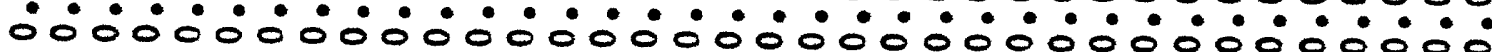
嚄

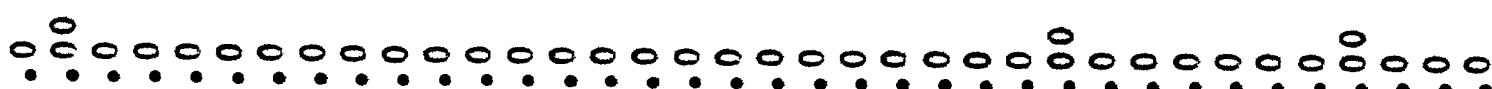

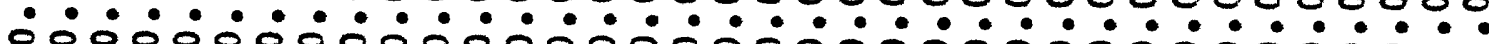
in 0000000000000000000000000000000000 ก

Ð

000080080000000000000000000080080000 0 000000000000000000000000000000000000

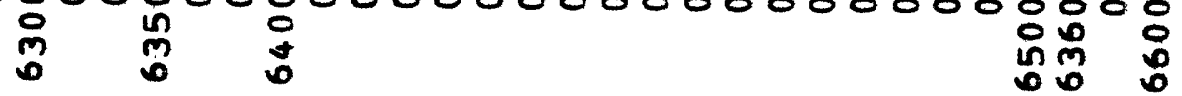

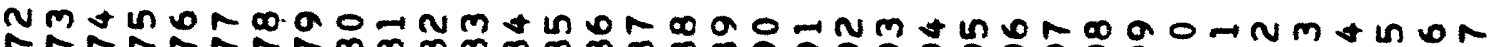

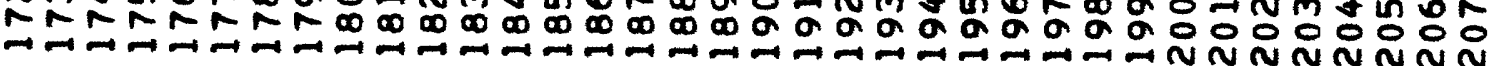

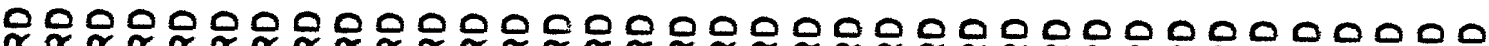

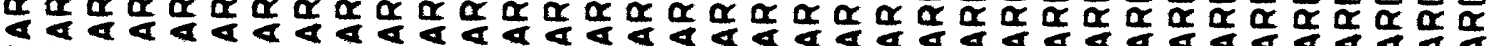

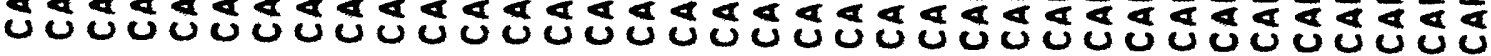


:0 :0

$\begin{array}{lll}0 & 0 \\ 0 & 0 & 0 \\ 0 & 0\end{array}$

000000000000000000000000000

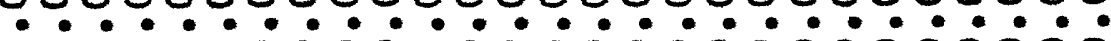
000000000000000000000000000

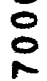

000000000000000000000000000

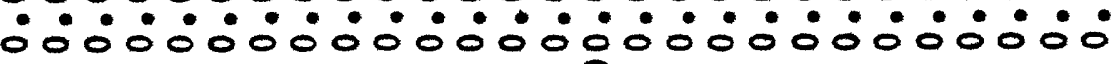
$\stackrel{-}{1}$

000008000000000000000000000

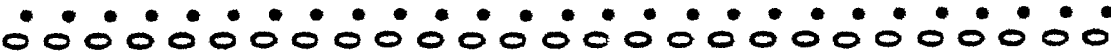
$00000800 \% 008000000000000000$ 000000000000000000000000000

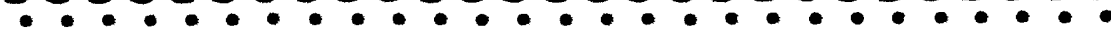

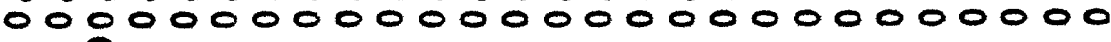
in

To

008000000000000000000000000

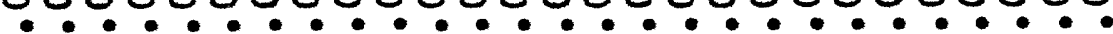
0000000000000000000000000 :

000000000000000000000000000

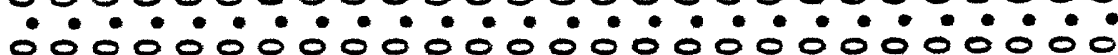

000000000000000000000000000 í

000000000000000000000000000

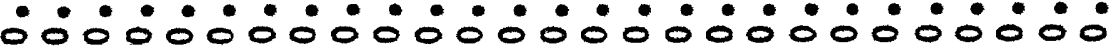

000000000000000000000000000

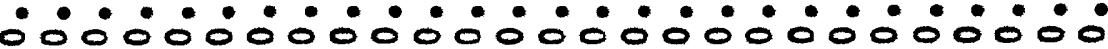

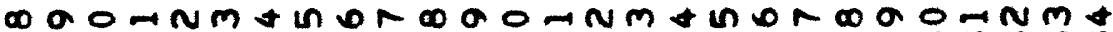

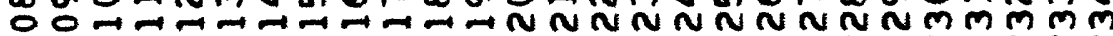

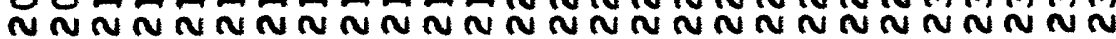

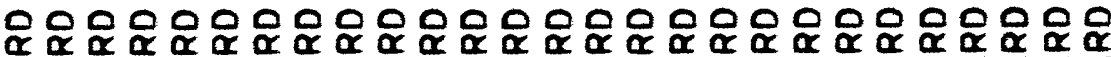

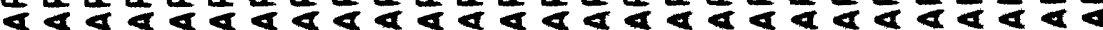

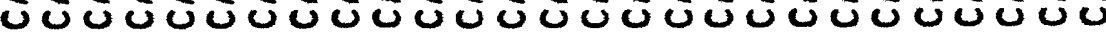




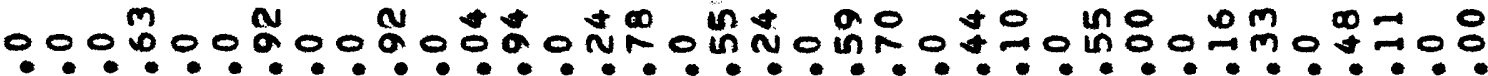

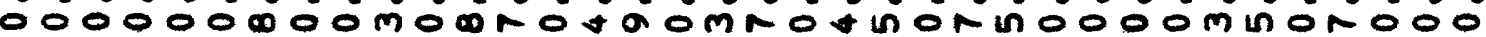

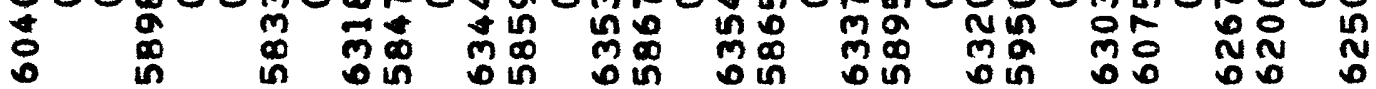

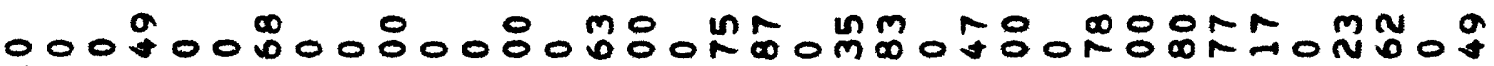
-

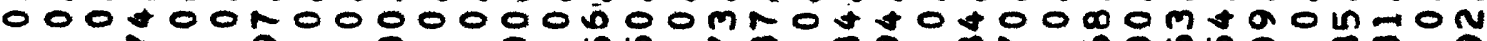

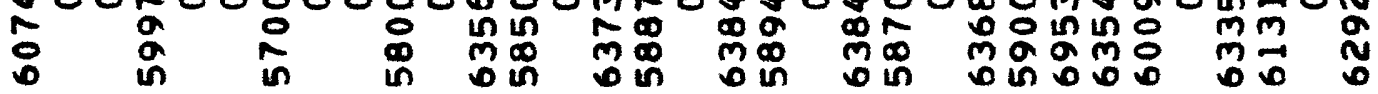

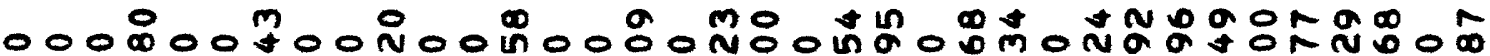
:

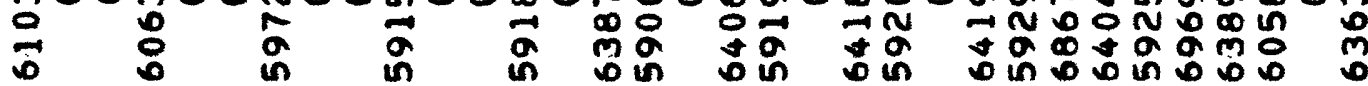
o 00 mo :00

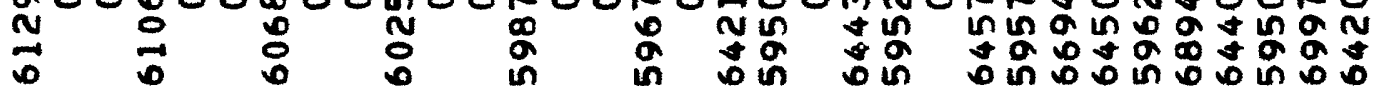

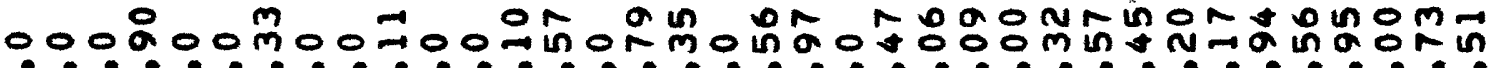

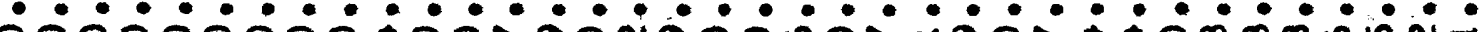

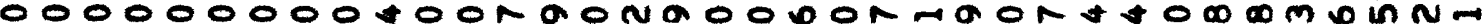

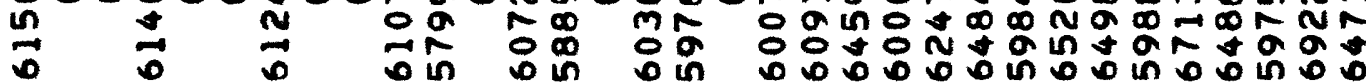

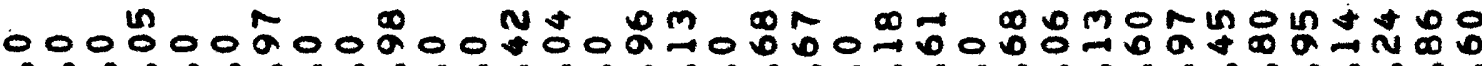

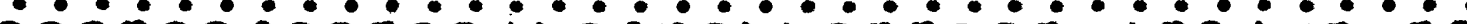

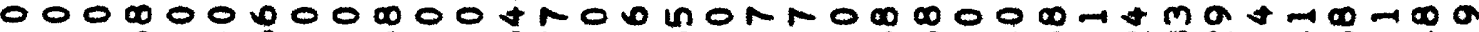

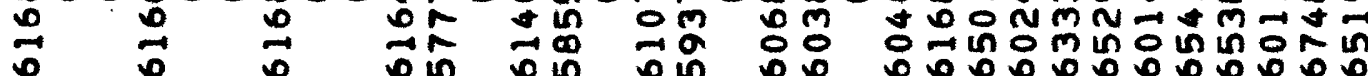
o o

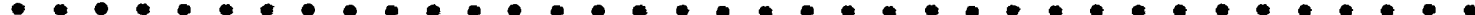

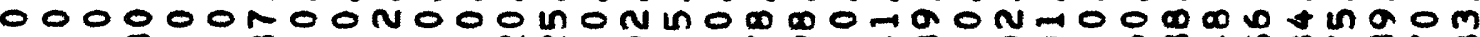

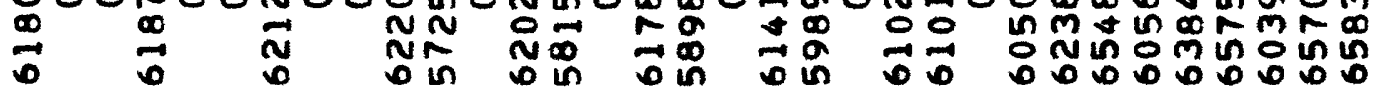

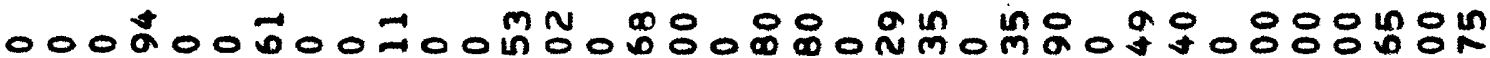
:

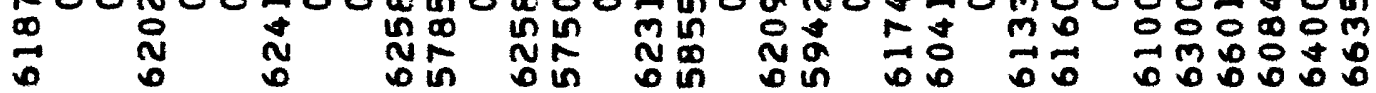
$0+0+m \rightarrow N 0$ o

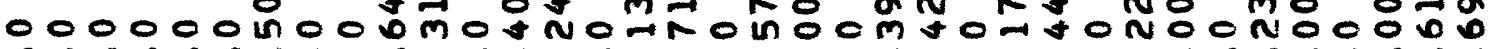

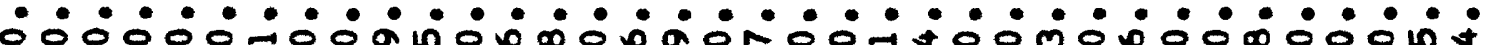

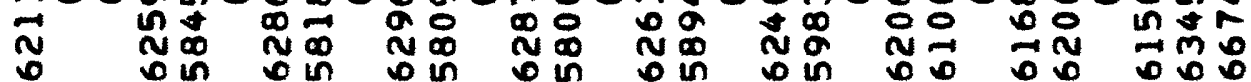
o o o o o o o o n mo \%

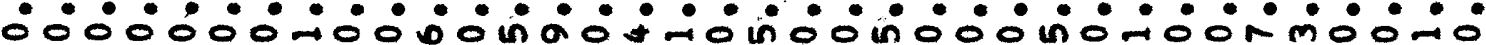

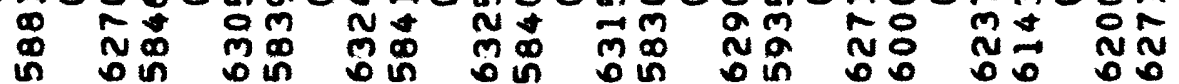

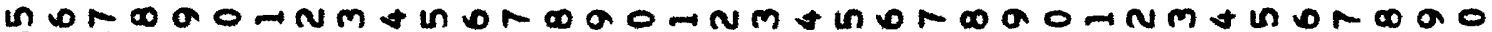

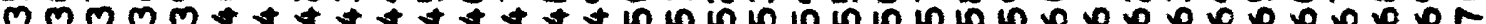

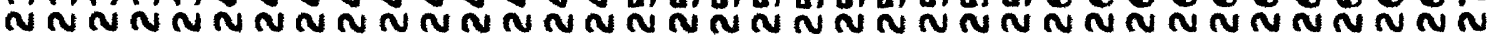

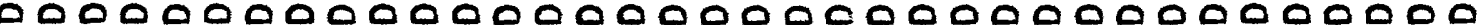

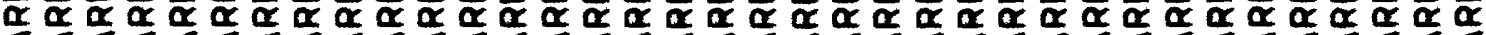

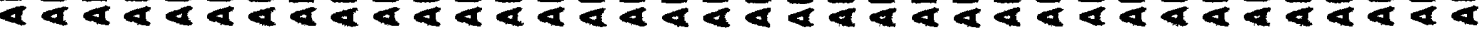

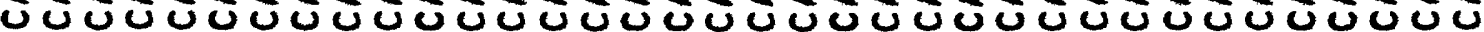




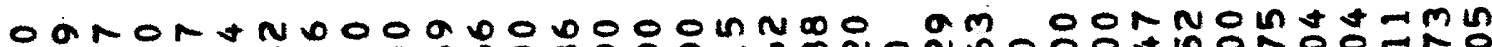
- ONN N : $m$ o a o m a

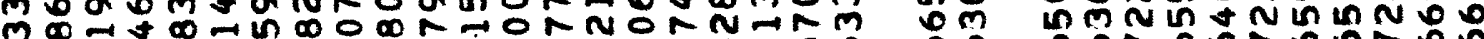

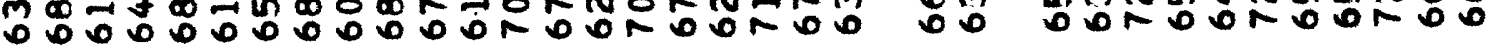

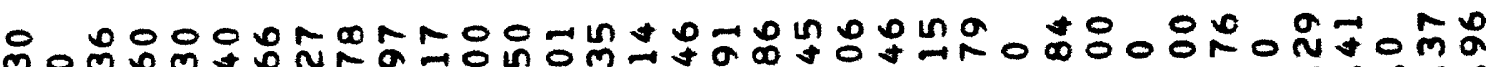

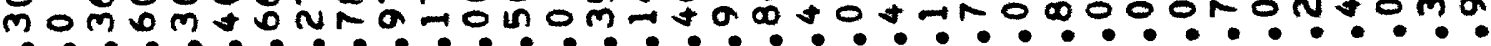
-

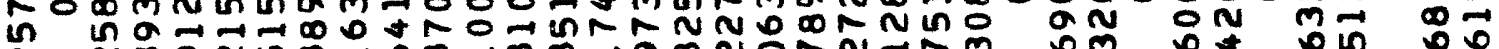

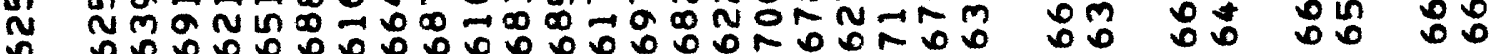

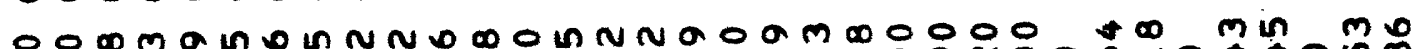

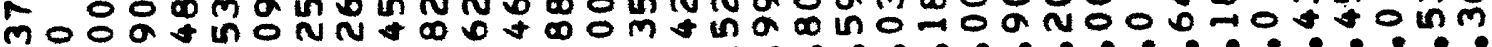
-

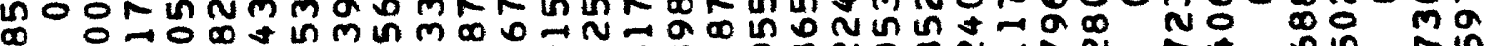

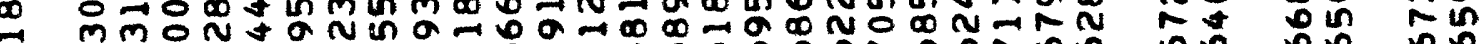

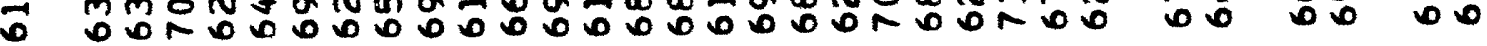

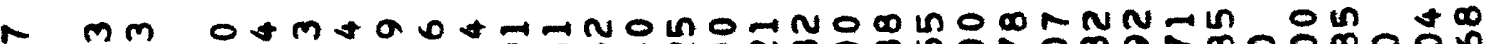

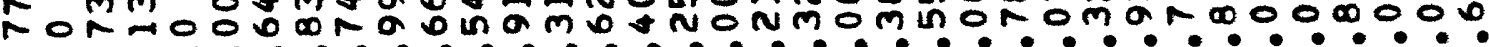
- 숭ㅇㅁ

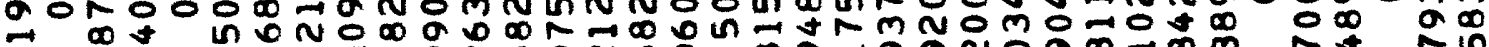

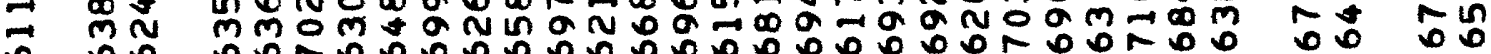

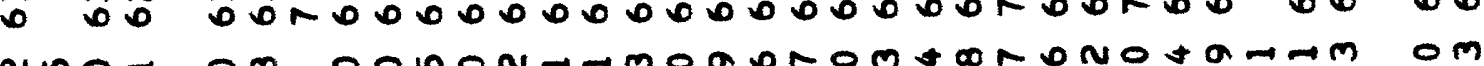
늉 - : : - : : - : - : +mN no ñ

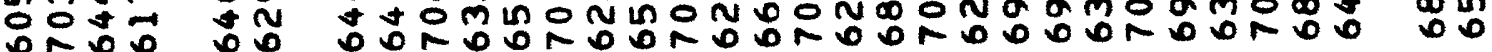

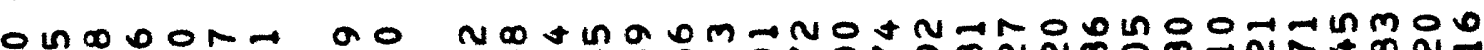

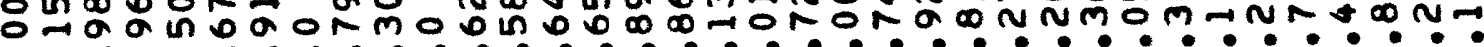

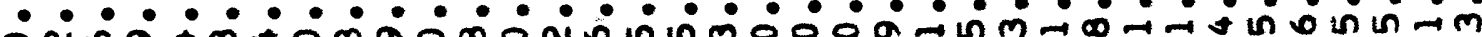
on $0+m+0 \infty a 0 \infty 0 N$ m

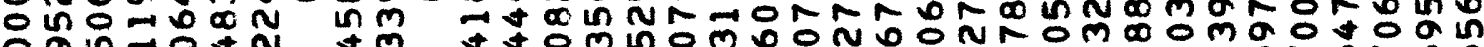

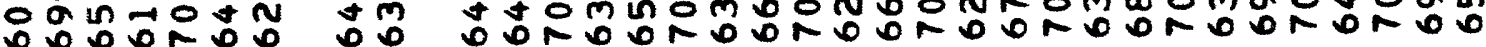

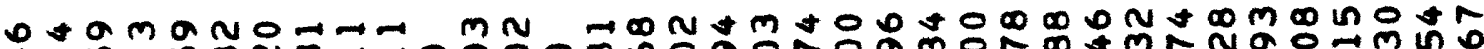

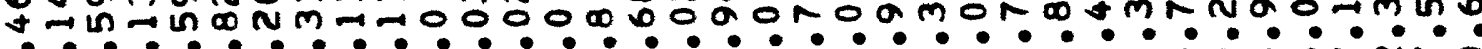

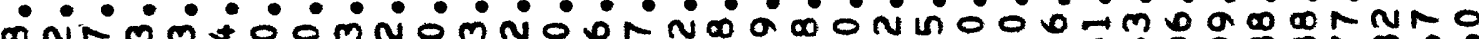

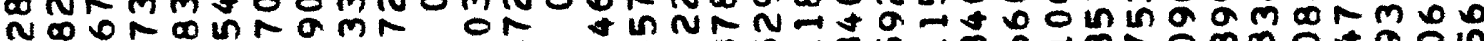

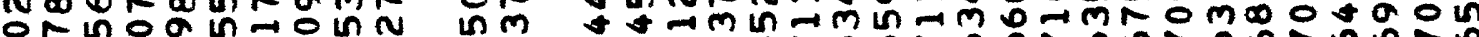

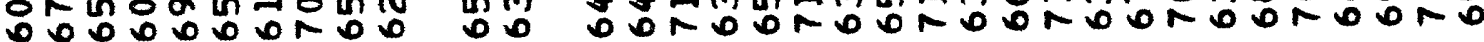

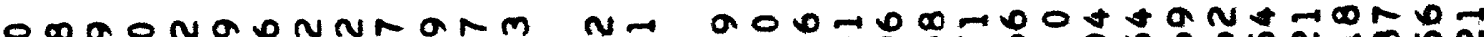

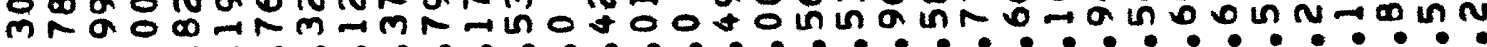
: in

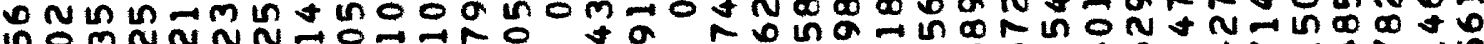

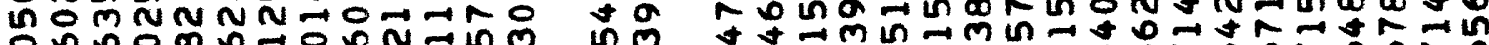
0000 Un

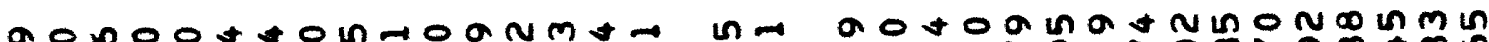

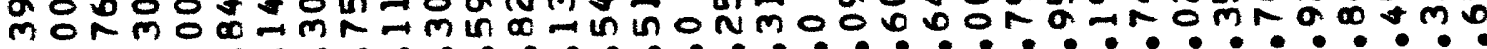

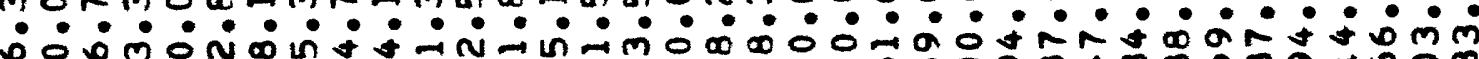

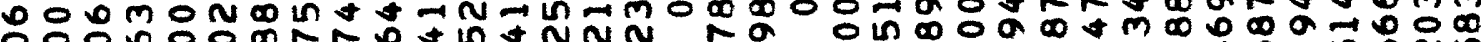

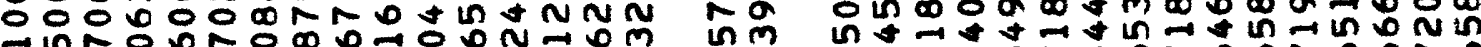

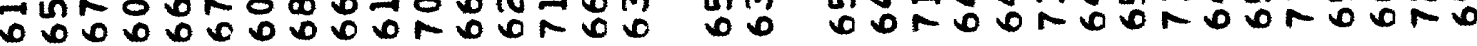

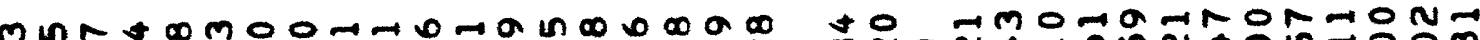

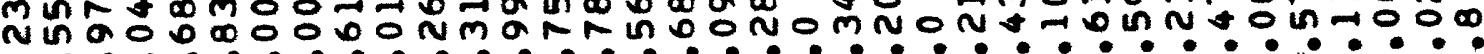
- com in

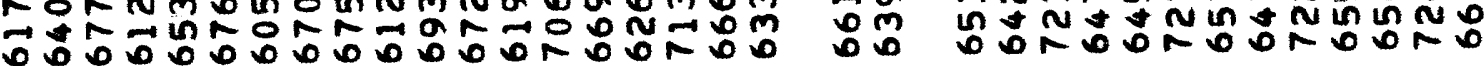

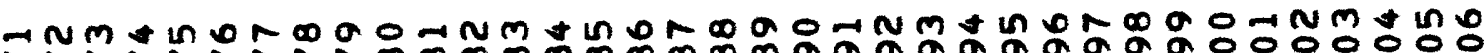
- T R R R R R

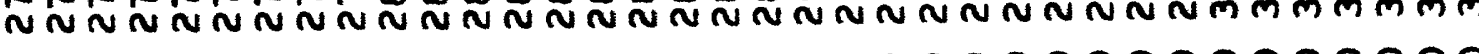

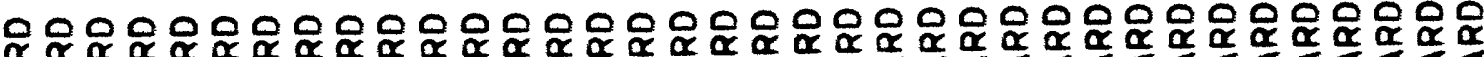

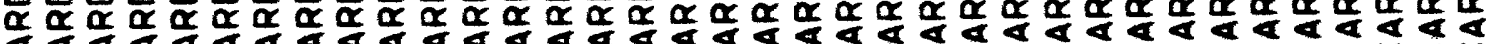
எ 


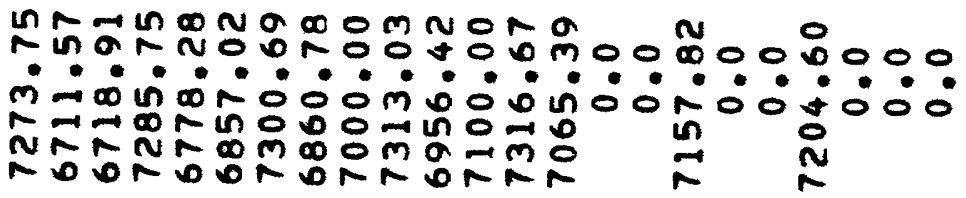

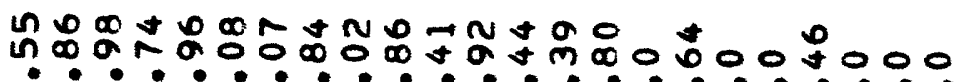
mín:

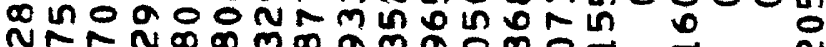

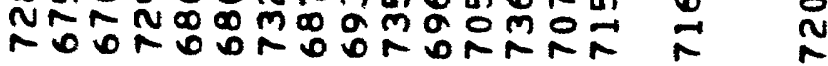

o

-

a a

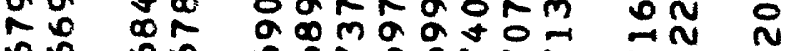

0 D 0 DDRDDRRAN N

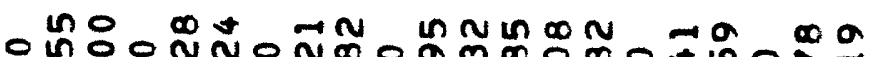

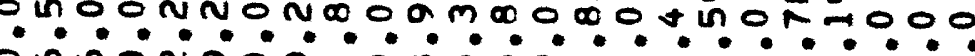

o o ona 0 a

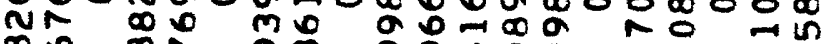

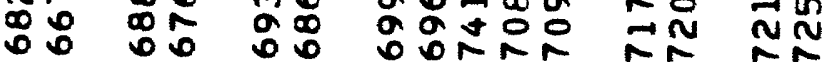

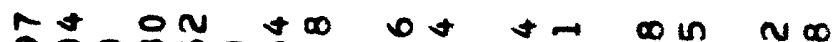

- a o o m in ino m morino

Óñ $\infty 0$ in $r+$ Nin or

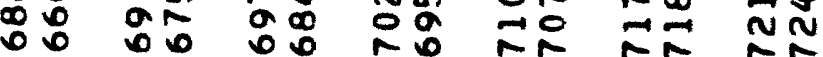

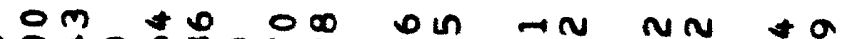

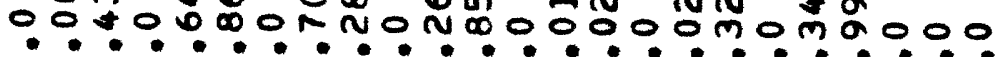

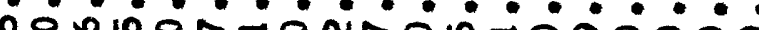

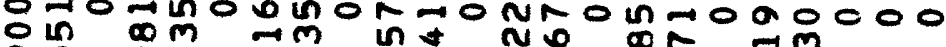

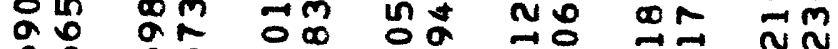

00 0 0 - 0 R 0 RN

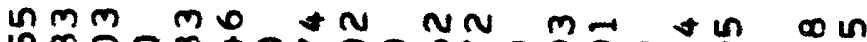

InOO

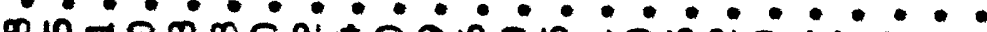

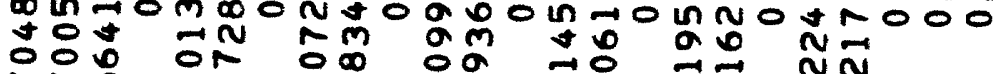

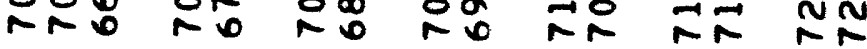

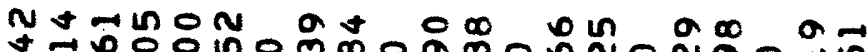

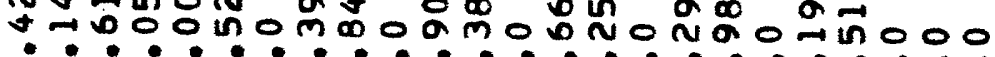

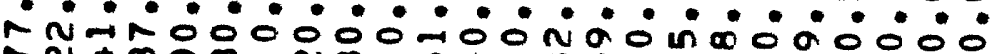

$r N \pm m 0 m$ N

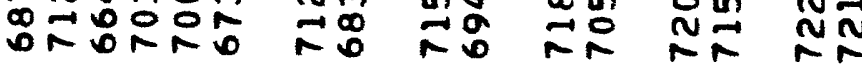

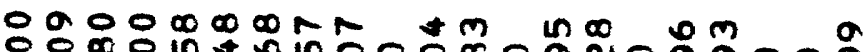

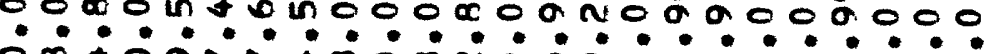

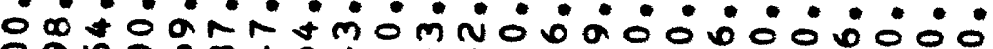
00 in 0 O

OND

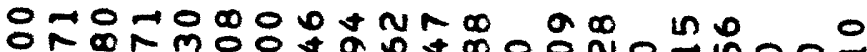

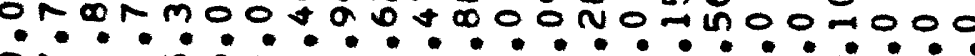

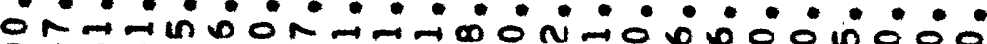

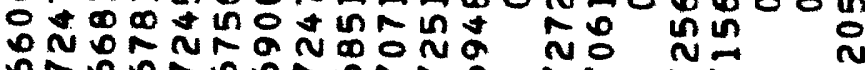
DND $0 N$ DN

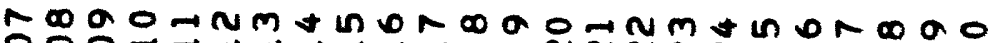

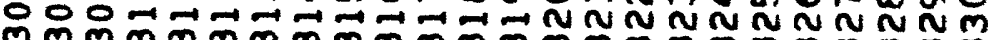
mलmmm 


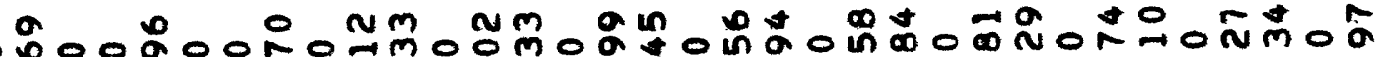

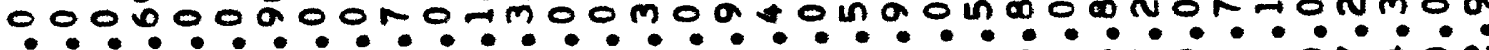

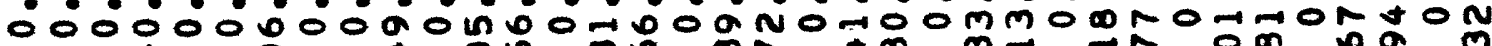

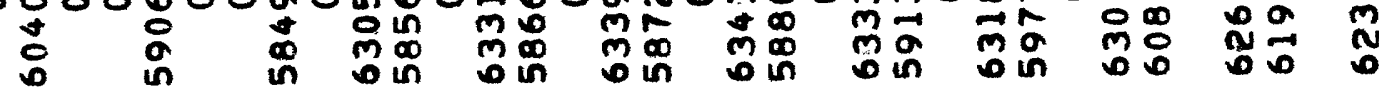

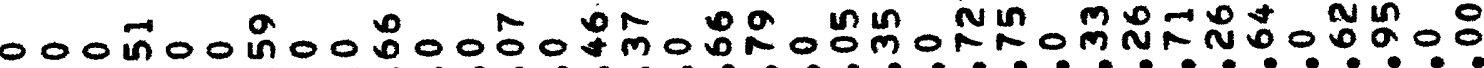

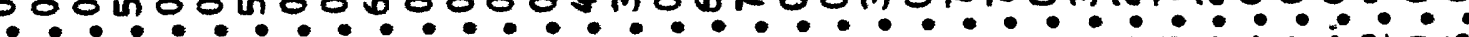

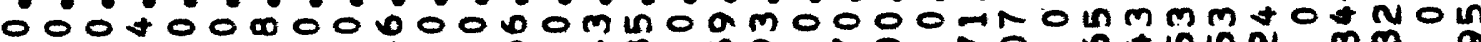

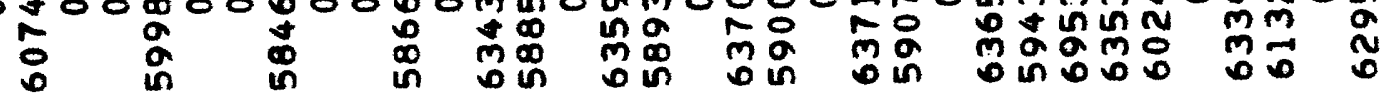

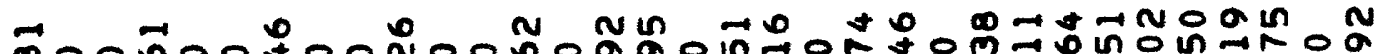

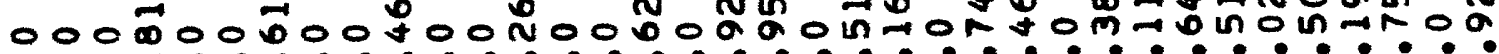
- ómó

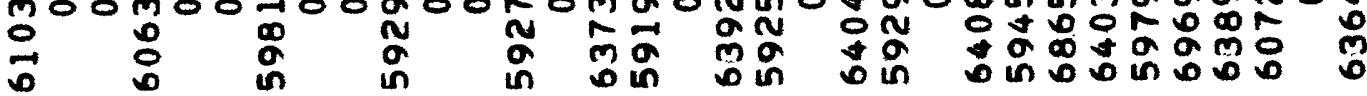

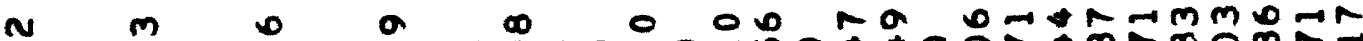

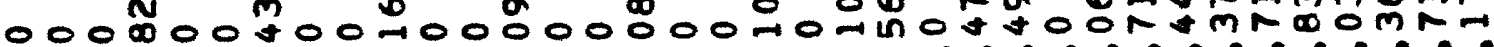

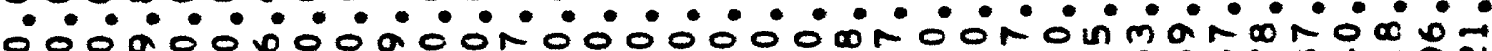

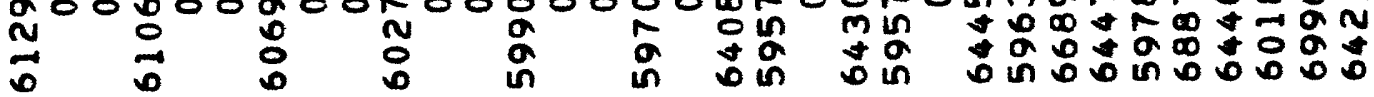
0 m

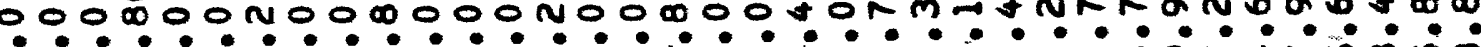
:0ं0ं0்

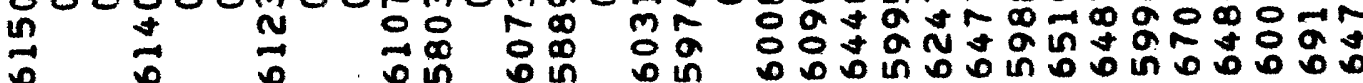
a 0 in 0 D

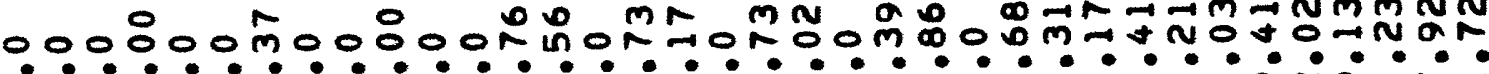

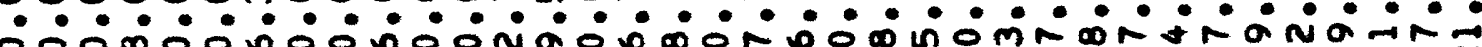

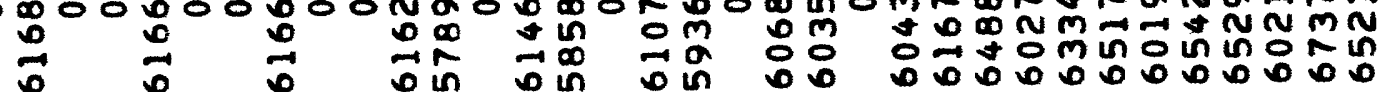

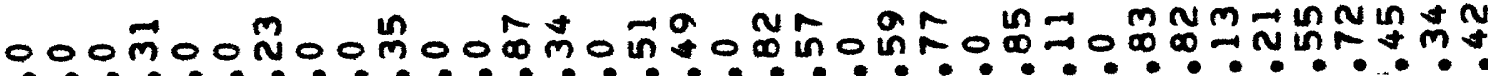

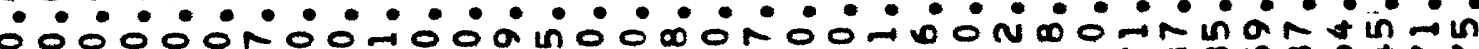

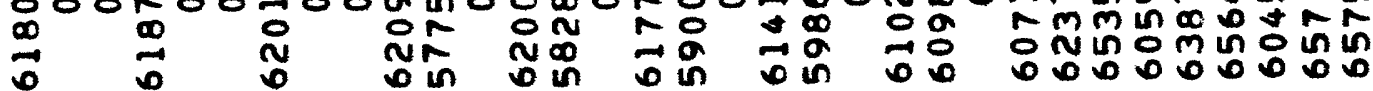

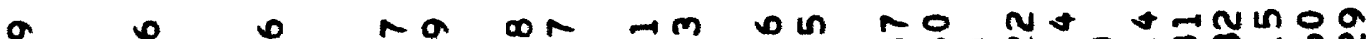

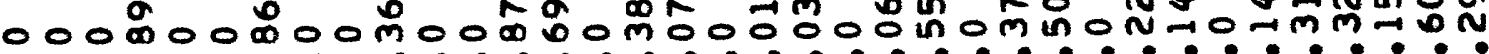
:

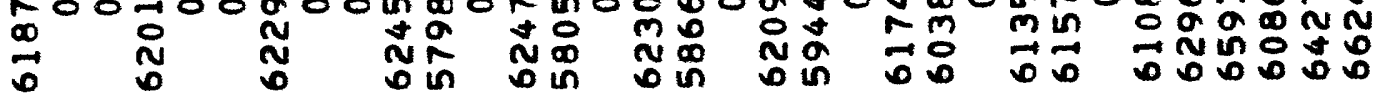

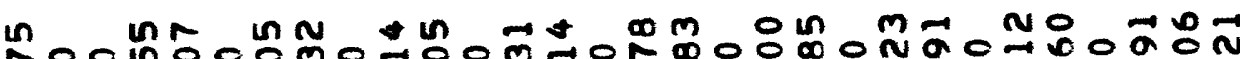

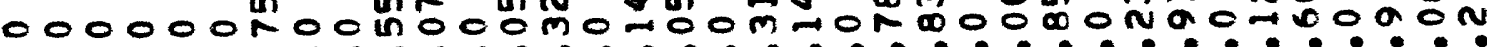
:

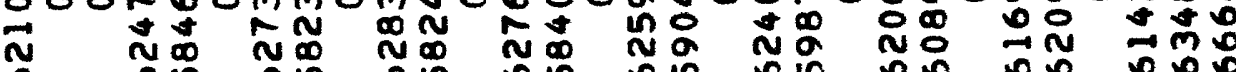

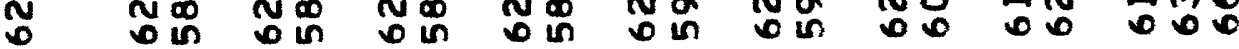

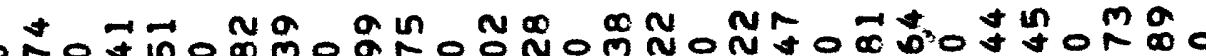
0000000 O O :

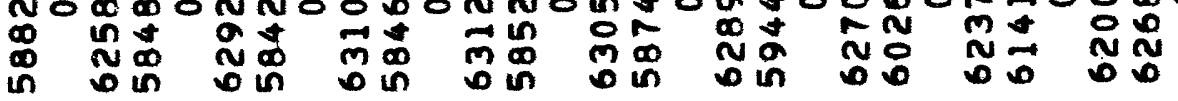

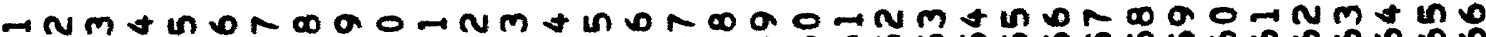

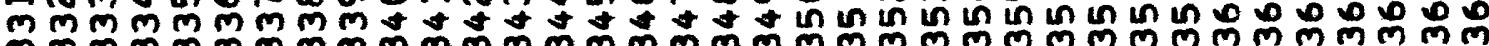

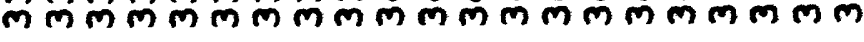

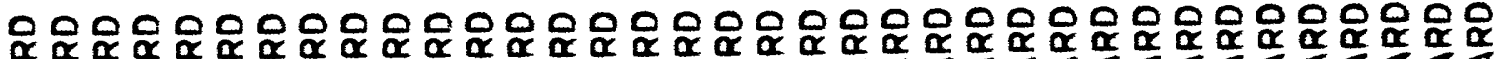

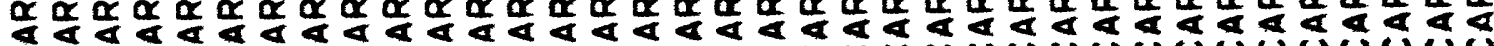

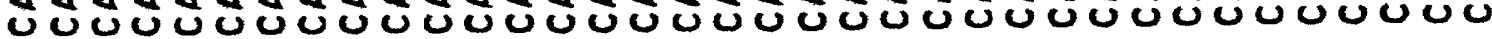




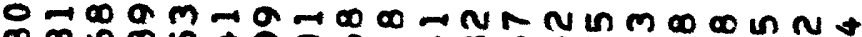

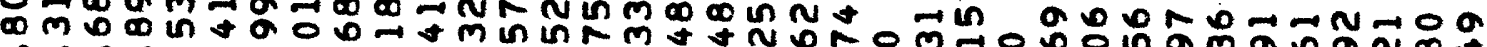

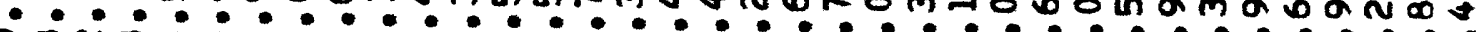

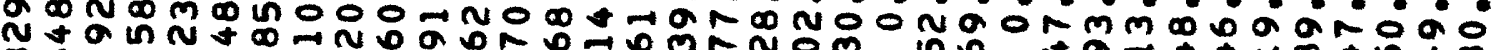

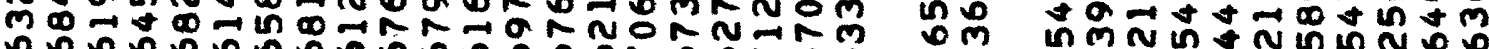

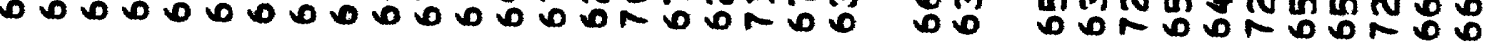

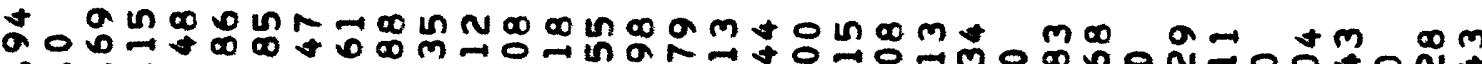

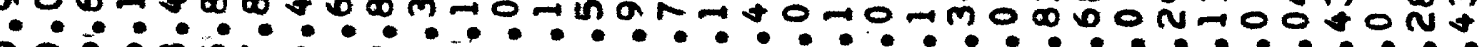

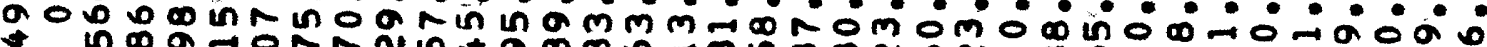

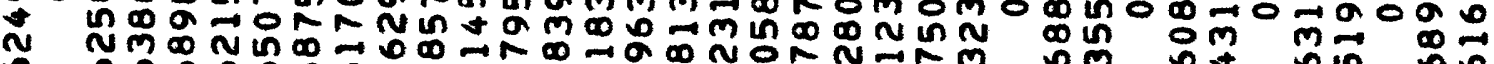

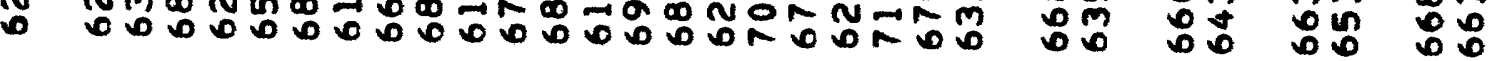

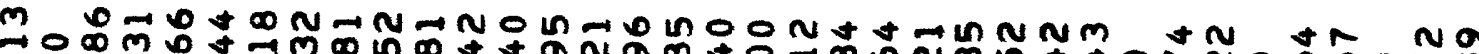

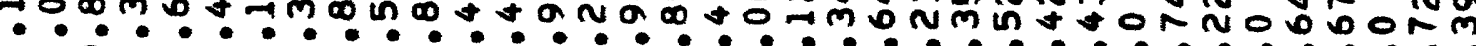

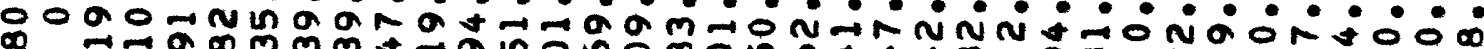

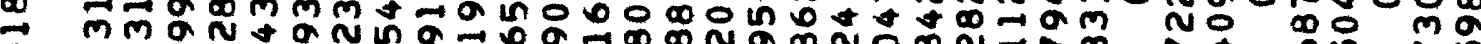
b

- Na onm n n $\quad$ n

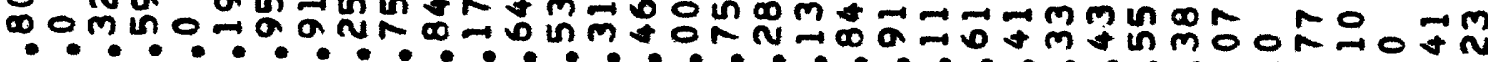

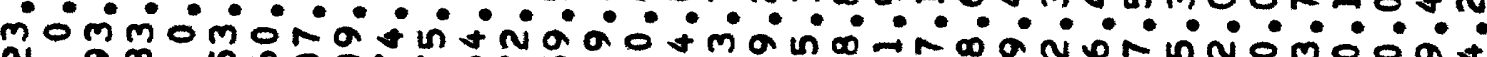

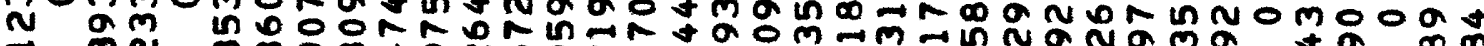

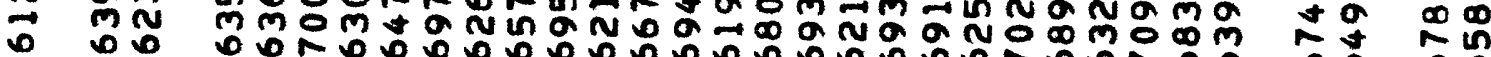
(

N Non OM

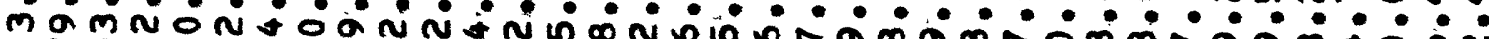

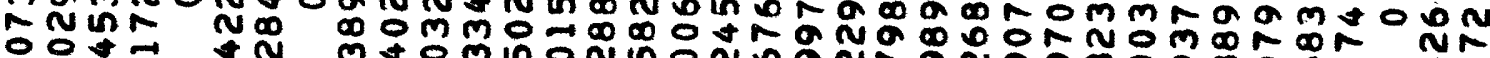
- 0 D

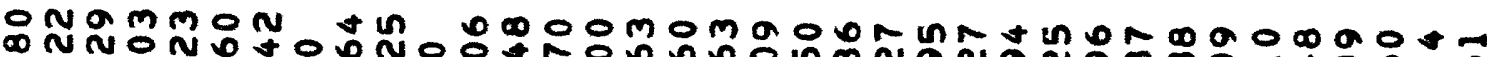

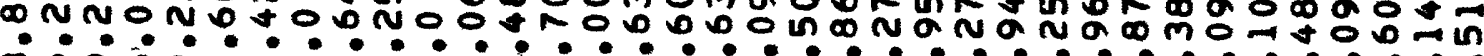

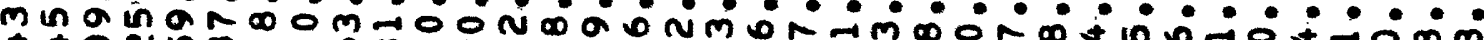

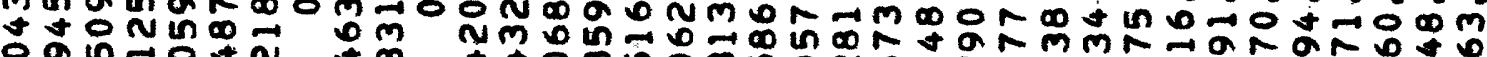

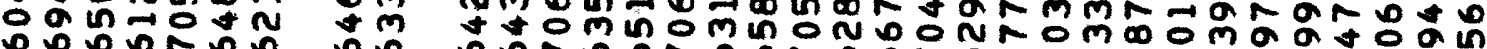

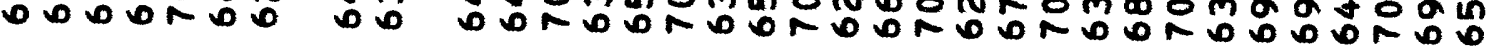

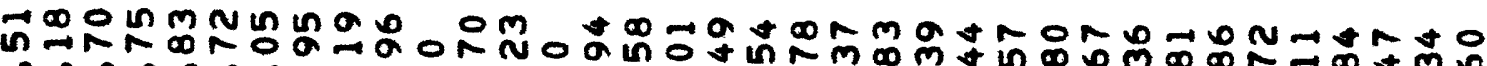
$\because$.

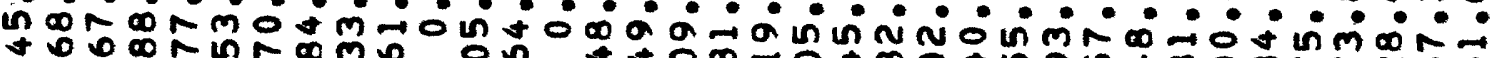

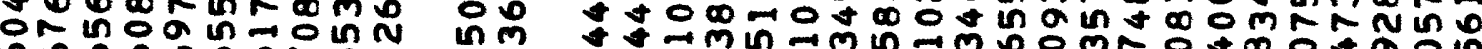

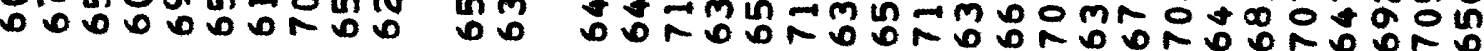

嗢

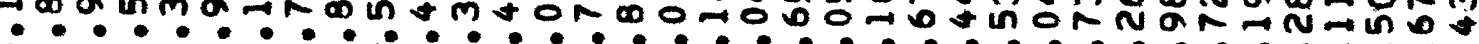

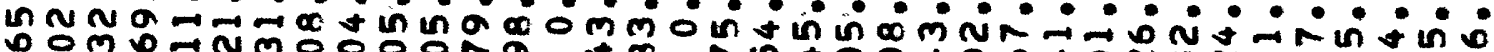

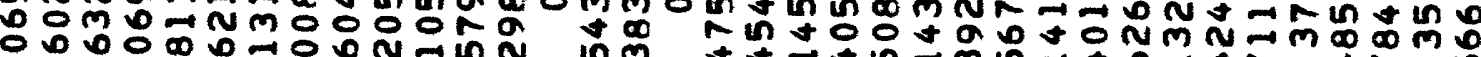

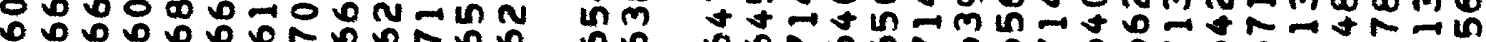
O

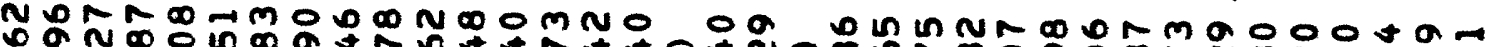

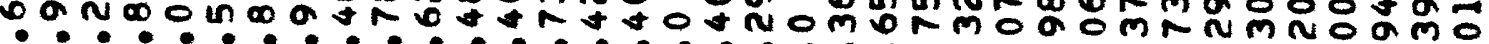

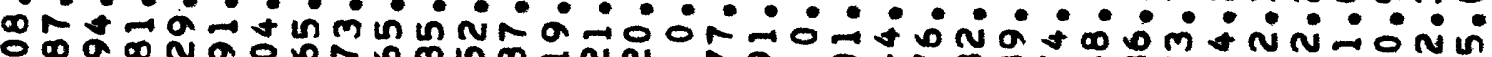

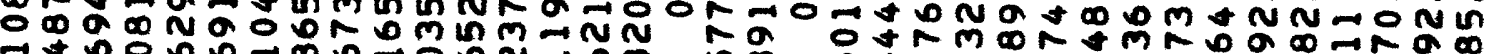

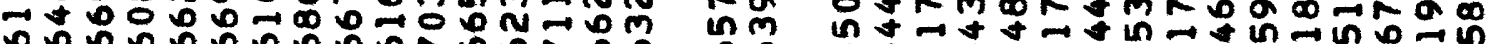

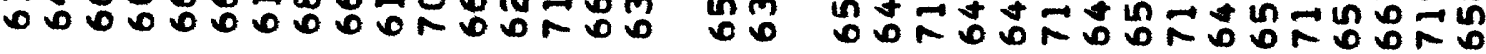

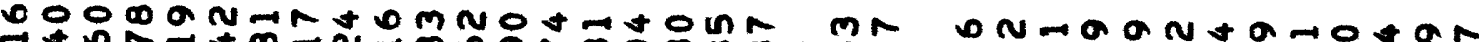

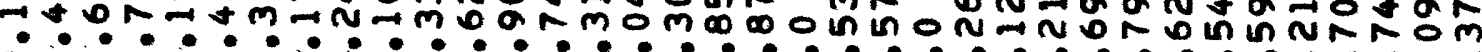

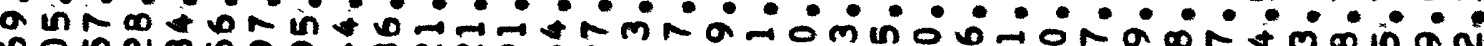
0 in 0 ina $\alpha$ m

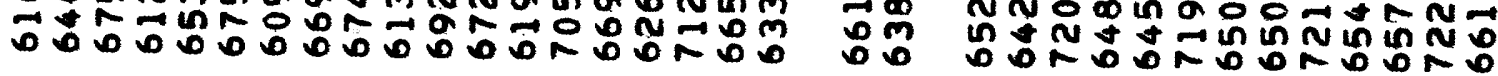

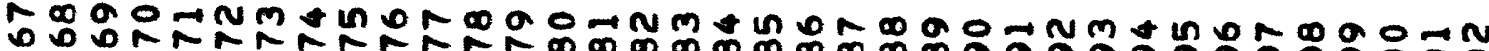
M

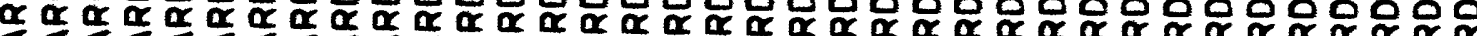

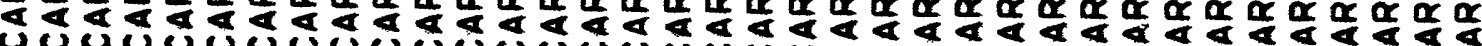
Uง 
a N

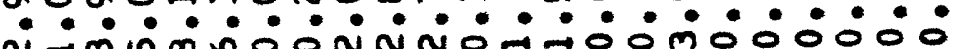

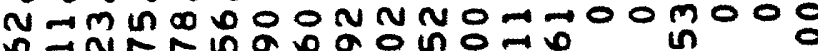

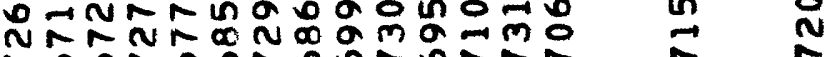

Non 0 D 0 N

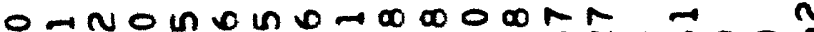

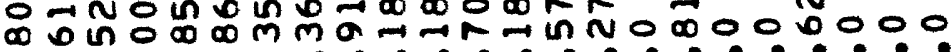

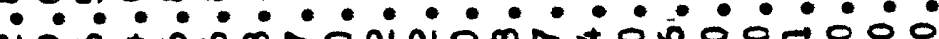

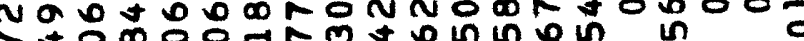

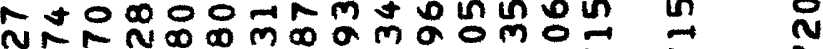

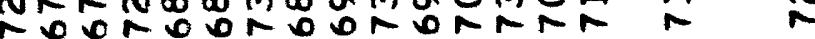

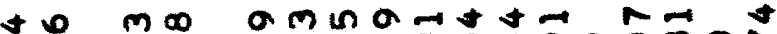

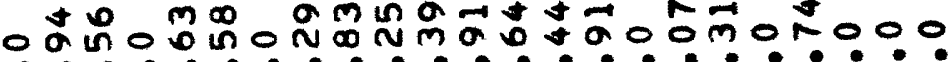

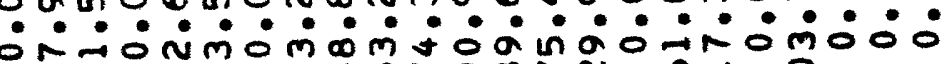

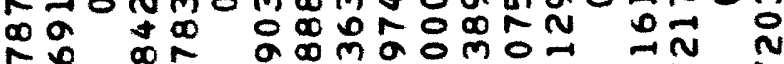

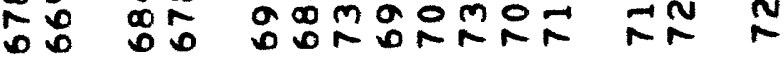

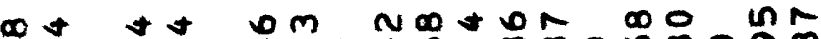

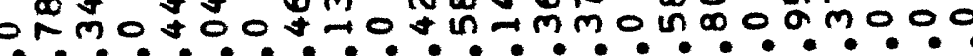

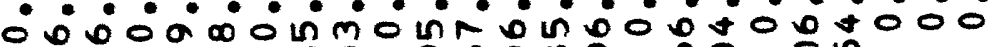

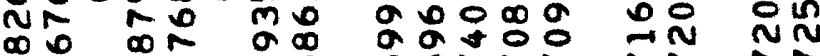

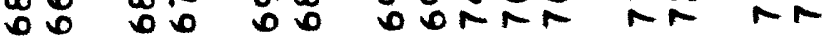

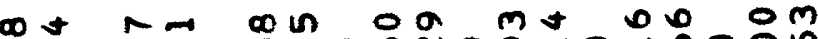

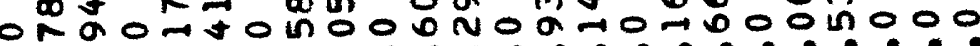

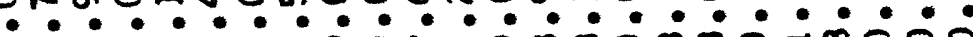

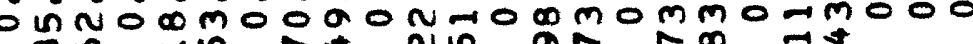

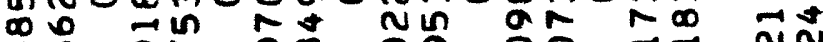

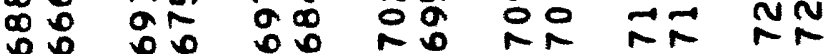

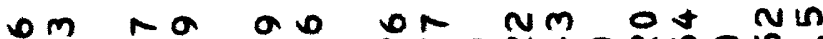

- ra o w r o n

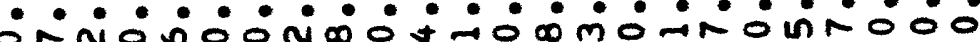

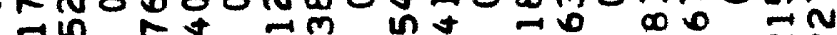

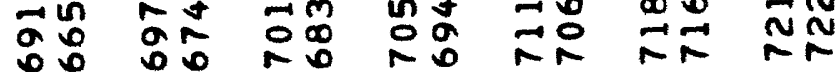

NOMNO

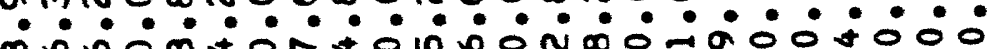

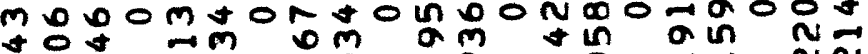

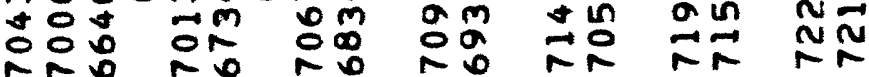

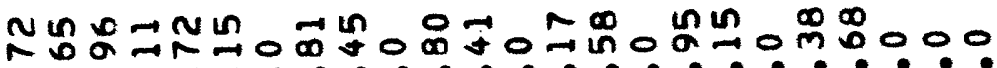

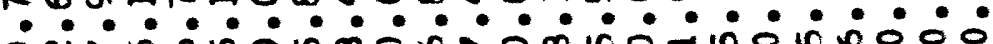

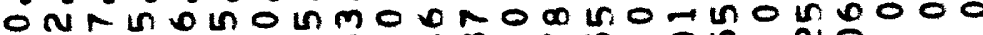

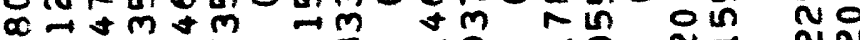

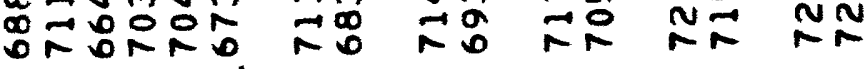

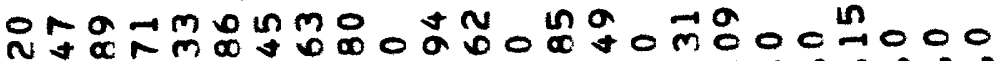
Ntarm. - manmminomomomomo

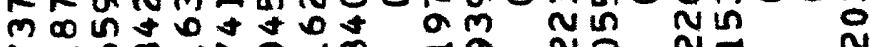

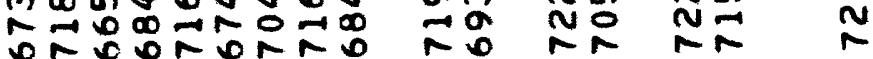

m

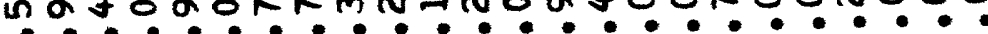

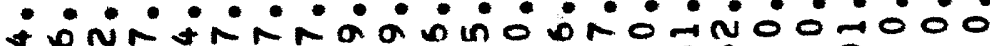

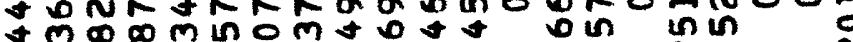
N 0 D

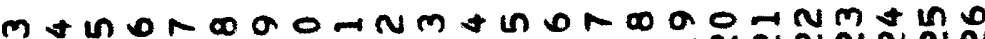

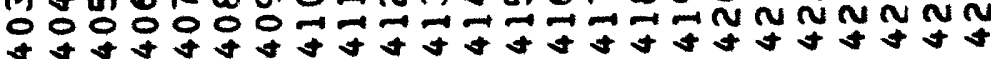

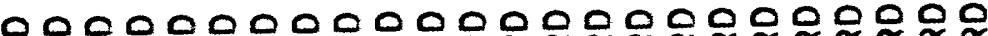

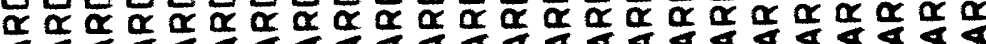

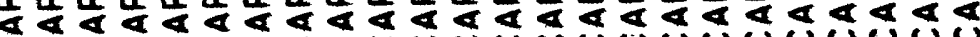


000000000000000000000000000000000

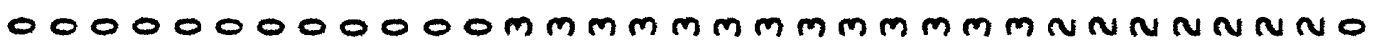
$0000000000 \mathrm{mmmmmmmmmmmmmmm000NNNNO}$ $00000000000 \mathrm{mmmmmmmmmmmmmoNNNNNNNO}$

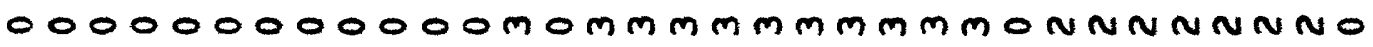

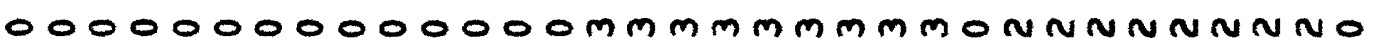

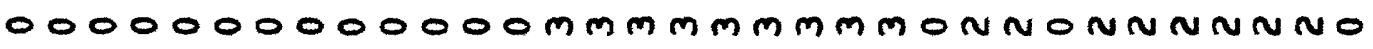

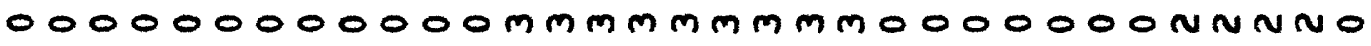

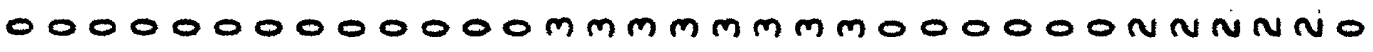

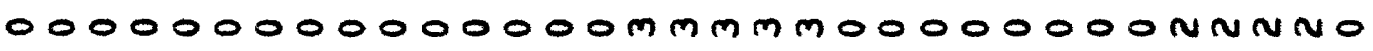
$000000000000000 \mathrm{~m} 0 \mathrm{~mm} 0000000 N N N N N 0$ $000000000000000000 m 00000000 n n N N n 0$ $0000000000000000000000000000 N N N N 0$ $00000000000000000000000000000 N N N 0$ $00000000000000000000000000 N N N N N N 0$ $0000000000000000000000000 N N N N N N n 0$ $0000000000000000000000000 N N N N N N 00$ $000000000000000000000000000 N N N N 00$ $000000000000000000000000 n n N N N N N 00$ $000000000000000000000 N N 000 N N N N 000$ $0000000000000000000000 N N N N N N N N 000$

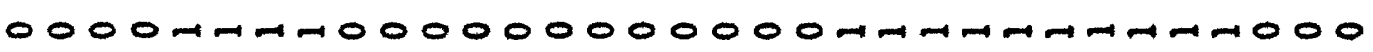

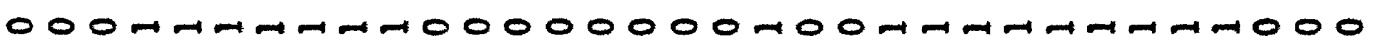
० 0 О

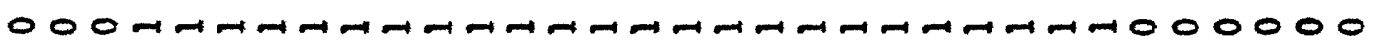

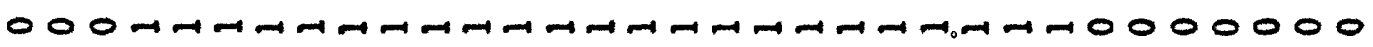

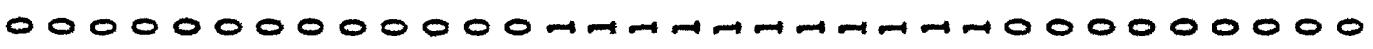
$00000000000000 M H A H A H A-0000000000$ 000000000000000000000000000000000

N

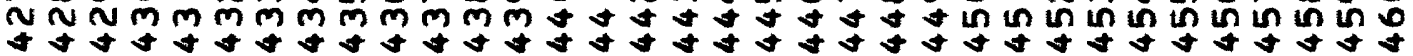

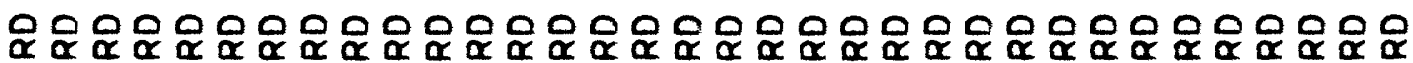

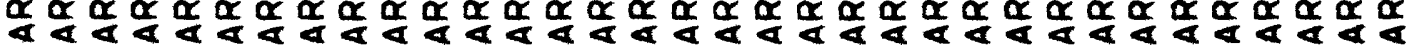

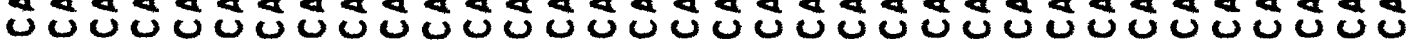


COMPUTER OUTPUT 
0000000000 : 0 0 0 :

0000000000 $\because 000000000$

0000000000 ட்ட்ட்ட்ட்0

0000000000 ல்

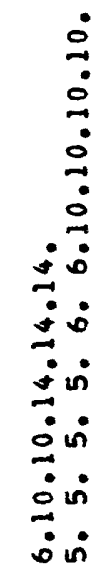

in in

0000000000 ०00000000:

in in

in เท

in in

in in

$000000000 \%$

in in

in in

in in

cin

0000000000 ப்:0்:0்:0:0

in

i in

$\dot{\infty}$ in

$\therefore$ ० 000000000
0000000000

$\therefore \circ$

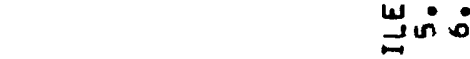

ज्ञ

w $\quad 0000000000$ I

in

o:

$2 \div$

ज००

a. :

in:

$\because \because$ 
$\dot{0} \dot{0} \dot{0} \dot{0} \dot{0} \dot{0} \dot{0} \dot{0} \dot{0} \dot{0} \dot{0} \dot{0} \dot{0} \dot{0} \dot{0} \dot{0}$ $\therefore \dot{0} \dot{0} \dot{0} \dot{0} \dot{i} \dot{i} \dot{i} \dot{i} \dot{i} \dot{i} \dot{i} \dot{i} \dot{i} \dot{i} \dot{i}$

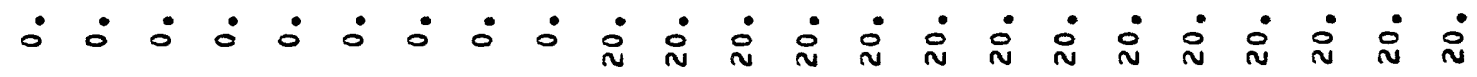
$\dot{0} \dot{0} \dot{0} \dot{0} \dot{i} \dot{i} \dot{i} \dot{i} \dot{i} \dot{i} \dot{i} \dot{i} \dot{i} \dot{i} \dot{i}$ $\dot{0} \dot{0} \dot{0} \dot{0} \dot{0} \dot{i} \dot{i} \dot{i} \dot{i} \dot{i} \dot{i} \dot{i} \dot{i} \dot{i} \dot{i} \dot{i}$

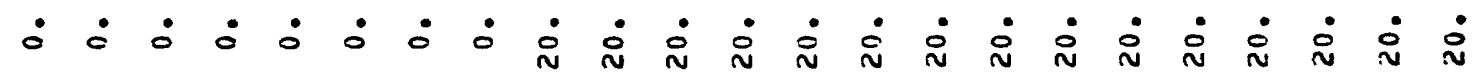

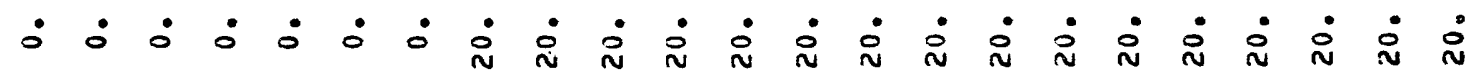

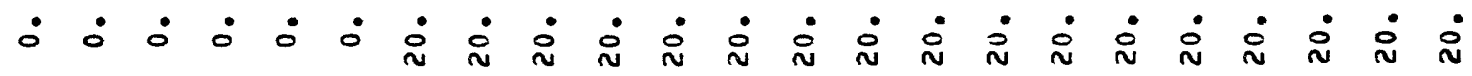
$\dot{0} \dot{0} \dot{0} \dot{i} \dot{i} \dot{i} \dot{i} \dot{i} \dot{i} \dot{i} \dot{i} \dot{i} \dot{i} \dot{i} \dot{i} \dot{i} \dot{i}$ $\dot{0} \dot{0} \dot{i} \dot{i} \dot{i} \dot{i} \dot{i} \dot{i} \dot{i} \dot{i} \dot{i} \dot{i} \dot{i} \dot{i} \dot{i} \dot{i} \dot{i}$ $\dot{0} \dot{0} \dot{i} \dot{i} \dot{i} \dot{i} \dot{i} \dot{i} \dot{i} \dot{i} \dot{i} \dot{i} \dot{i} \dot{i} \dot{i} \dot{i} \dot{i}$

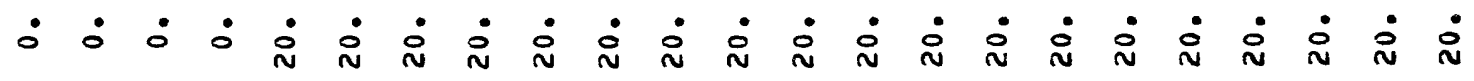
$\dot{0} \dot{i} \dot{i} \dot{i} \dot{i} \dot{i} \dot{i} \dot{i} \dot{i} \dot{i} \dot{i} \dot{i} \dot{i} \dot{i} \dot{i} \dot{i} \dot{i}$

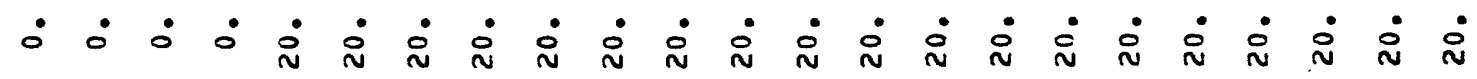
$\dot{0} \dot{i} \dot{i} \dot{i} \dot{i} \dot{i} \dot{i} \dot{i} \dot{i} \dot{i} \dot{i} \dot{i} \dot{i} \dot{i} \dot{i} \dot{i} \dot{i}$

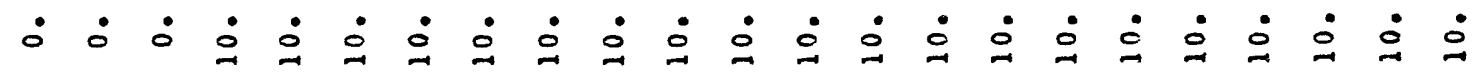

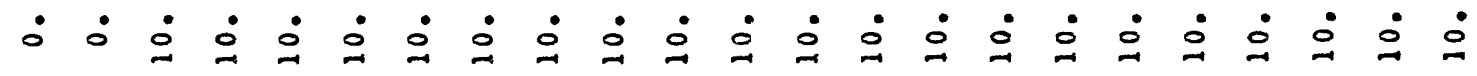

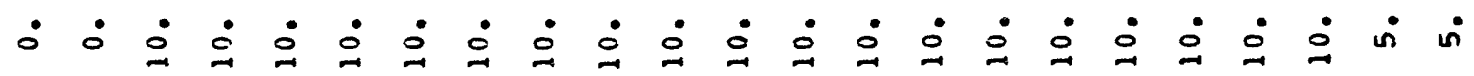

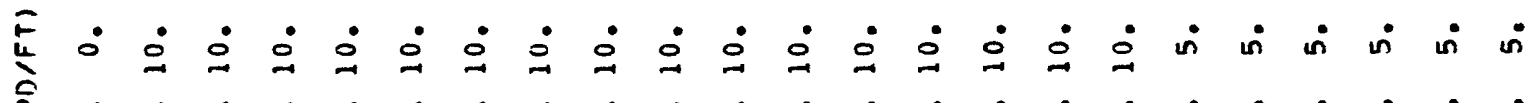

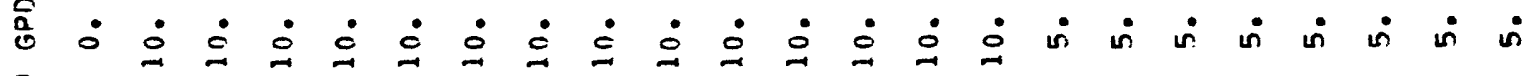

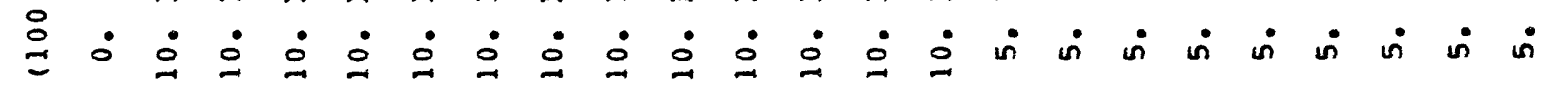

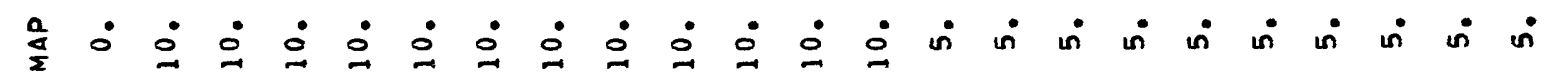

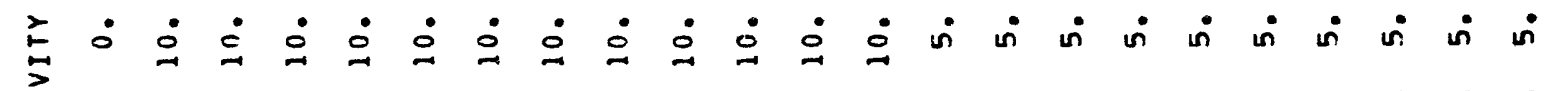

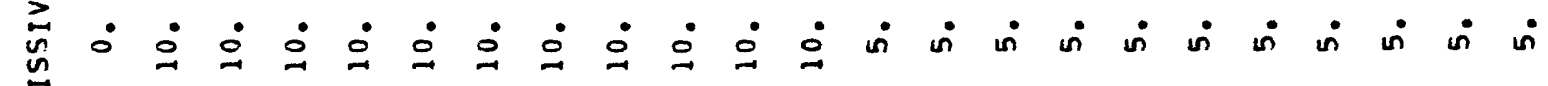

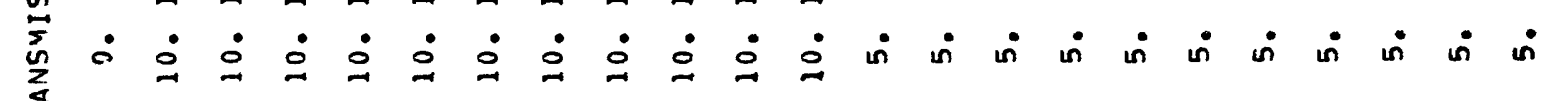

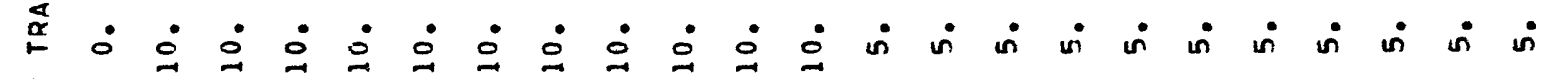

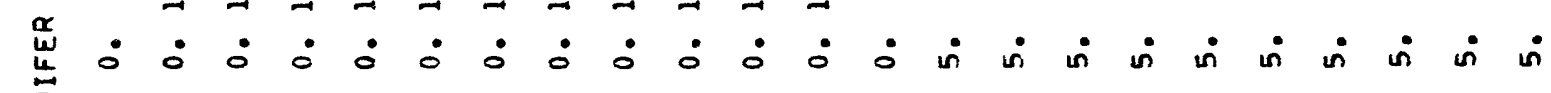

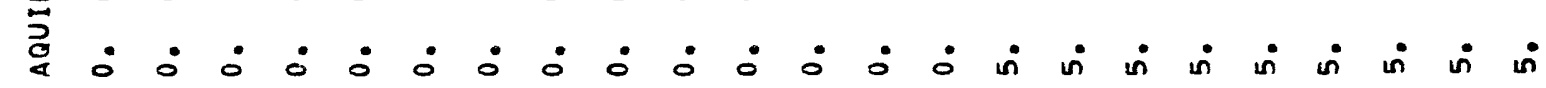

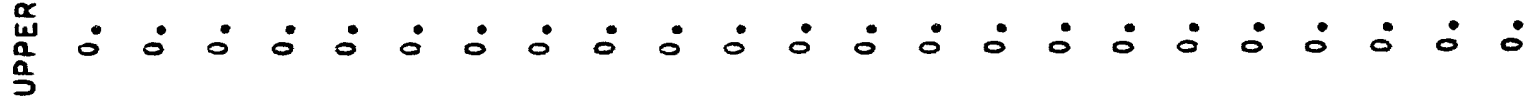


$\dot{0} \dot{0} \dot{0} \dot{0} \dot{0} \dot{0} \dot{0}$

$\dot{i} \dot{i} \dot{i} \dot{i} \dot{i} \dot{i} \dot{0}$

$\dot{\sim} \dot{i} \dot{i} \dot{i} \dot{\sim} \dot{i} \dot{0}$

$\dot{i} \dot{i} \dot{i} \dot{i} \dot{i} \dot{i} \dot{0}$

$\dot{\sim} \dot{i} \dot{i} \dot{i} \dot{i} \dot{i} \dot{0}$

$\dot{i} \dot{i} \dot{i} \dot{i} \dot{i} \dot{i} \dot{0}$

$\dot{\sim} \dot{\sim} \dot{\sim} \dot{\sim} \dot{i} \dot{i} \dot{0} \dot{0}$

$\dot{\sim} \dot{\sim} \dot{\sim} \dot{\sim} \dot{i} \dot{i} \dot{0}$

$\dot{i} \dot{\sim} \dot{\sim} \dot{i} \dot{\vdots} \dot{\vdots} \dot{0}$

$\dot{i} \dot{i} \dot{i} \dot{0} \dot{0} \dot{0} \dot{0}$

$\dot{\sim} \dot{\sim} \dot{\sim} \dot{\therefore} \dot{\therefore} \dot{0} \dot{0}$

$\dot{i} \dot{i} \dot{i} \dot{0} \dot{0} \dot{0} \dot{0}$

$\dot{i} \dot{\sim} \dot{\therefore} \dot{0} \dot{0} \dot{0} \dot{0}$

$\dot{\circ} \dot{\therefore} \dot{\circ} \dot{\therefore} \dot{\therefore} \dot{0}$

$\dot{\circ} \dot{\therefore} \dot{\circ} \dot{\circ} \dot{\circ} \dot{0}$

$\dot{\circ} \dot{\circ} \dot{\circ} \dot{\circ} \dot{\circ} \dot{0}$

$\dot{\circ} \dot{\vdots} \dot{\vdots} \dot{\circ} \dot{\circ} \dot{0} \dot{0}$

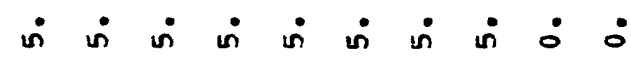

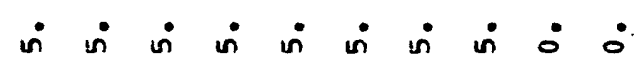

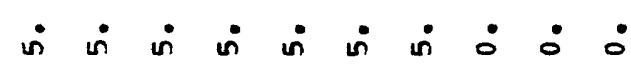

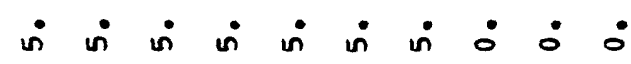

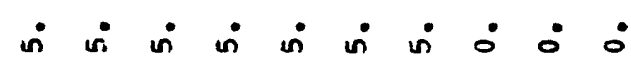

$\dot{n}$ in $\dot{n}$ in in in $\dot{0} \dot{0}:$

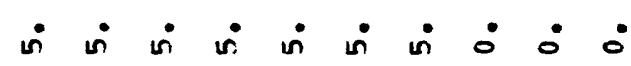

in $\dot{ }$ in $\dot{0} \dot{0} \dot{0} \dot{0}:$

in in in $\dot{0} \dot{0} \dot{0} \dot{0}$

in $\dot{0} \dot{0} \dot{0} \dot{0} \dot{0}$

$\dot{0} \dot{0} \dot{0} \dot{0} \dot{0} \dot{0}$

$\dot{0} \dot{0} \dot{0} \dot{0} \dot{0} \dot{0}$ 
$\dot{0} \dot{0} \dot{0} \dot{0} \dot{0} \dot{0} \dot{0} \dot{0} \dot{0} \dot{0} \dot{0} \dot{0} \dot{0} \dot{0} \dot{0} \dot{0} \dot{0}$

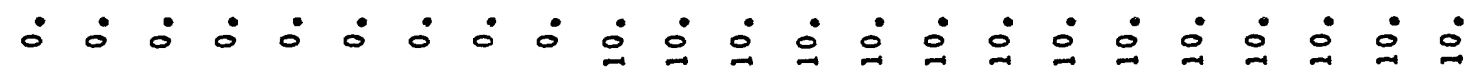

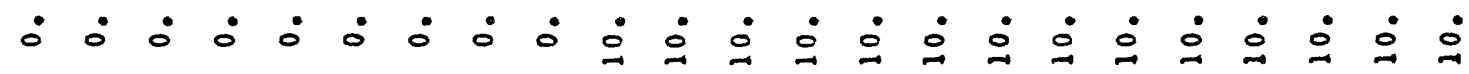

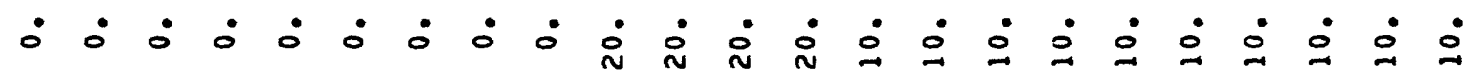
$\dot{0} \dot{0} \dot{0} \dot{0} \dot{0} \dot{i} \dot{i} \dot{i} \dot{i} \dot{i} \dot{i} \dot{i} \dot{i} \dot{i} \dot{i}$

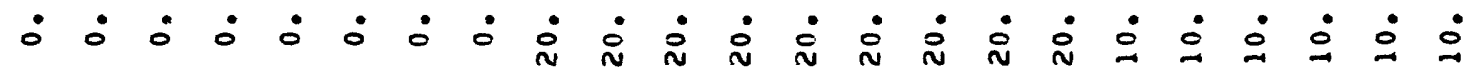

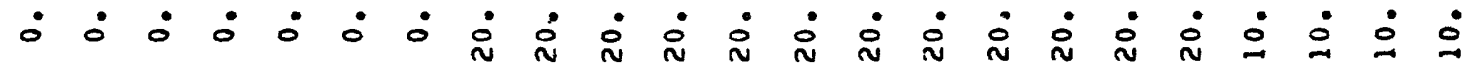
$\therefore \dot{0} \dot{0} \dot{i} \dot{i} \dot{i} \dot{i} \dot{i} \dot{i} \dot{i} \dot{i} \dot{i} \dot{i} \dot{i}$ $\dot{0} \dot{0} \dot{0} \dot{j} \dot{m} \dot{m} \dot{m} \dot{m} \dot{m} \dot{m} \dot{i} \dot{i} \dot{i} \dot{i} \dot{\sim} \dot{\sim} \dot{\sim} \dot{i}$ $\dot{0} \dot{0} \dot{0} \dot{m} \dot{m} \dot{m} \dot{m} \dot{m} \dot{m} \dot{m} \dot{i} \dot{i} \dot{i} \dot{i} \dot{i} \dot{i} \dot{i} \dot{i}$ $\dot{0} \dot{0} \dot{j} \dot{m} \dot{m} \dot{m} \dot{m} \dot{m} \dot{m} \dot{m} \dot{m} \dot{m} \dot{i} \dot{i} \dot{i} \dot{i} \dot{i} \dot{i}$ $\dot{0} \dot{0} \dot{0} \dot{m} \dot{m} \dot{m} \dot{m} \dot{m} \dot{m} \dot{m} \dot{m} \dot{m} \dot{m} \dot{m} \dot{i} \dot{i} \dot{i} \dot{i} \dot{i}$ $\dot{0} \dot{0} \dot{0} \dot{m} \dot{m} \dot{m} \dot{m} \dot{m} \dot{m} \dot{m} \dot{m} \dot{m} \dot{m} \dot{m} \dot{i} \dot{i} \dot{i} \dot{i}$ $\dot{0} \dot{0} \dot{0} \dot{j} \dot{j} \dot{j} \dot{j} \dot{j} \dot{m} \dot{m} \dot{m} \dot{m} \dot{m} \dot{m} \dot{m} \dot{m} \dot{i} \dot{i} \dot{i} \dot{i}$

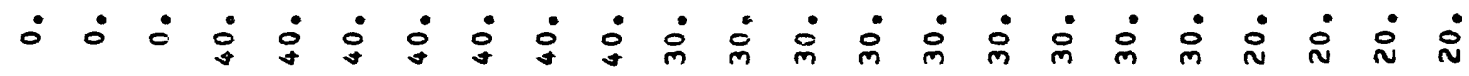
$\dot{0} \dot{0} \dot{j} \dot{j} \dot{i} \dot{i} \dot{i} \dot{q} \dot{q} \dot{q} \dot{j} \dot{m} \dot{m} \dot{m} \dot{m} \dot{m} \dot{m} \dot{i} \dot{i}$

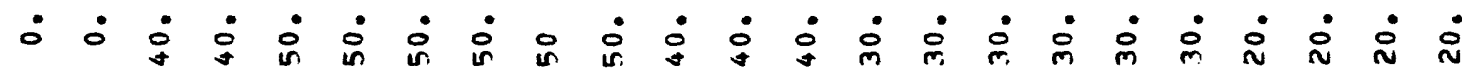

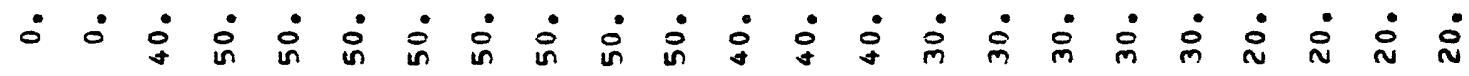

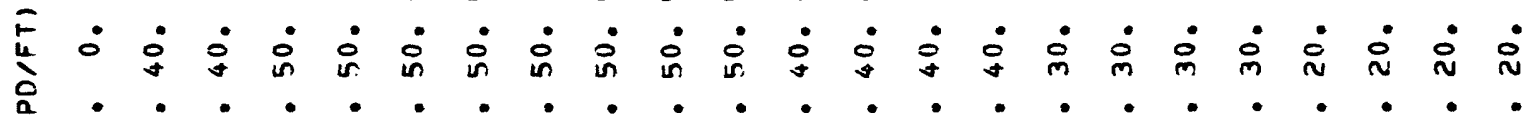

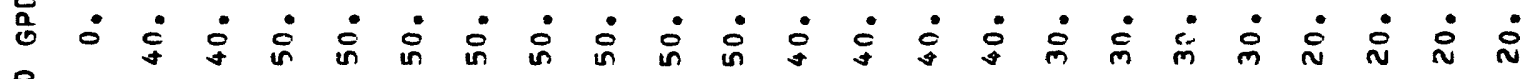

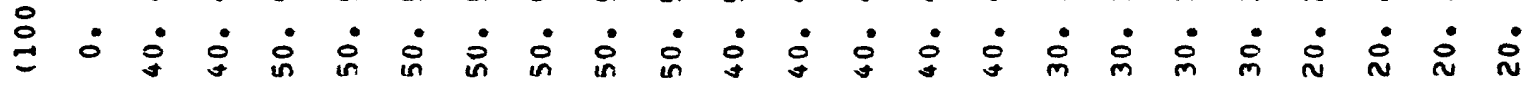

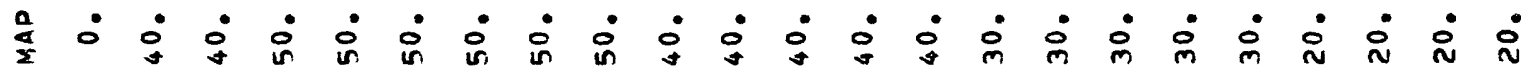

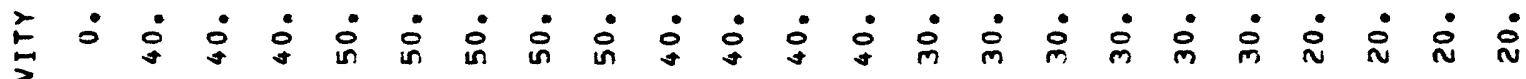

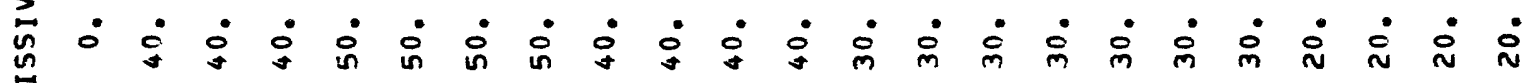

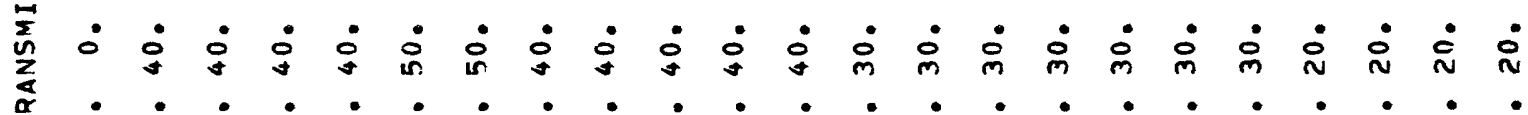

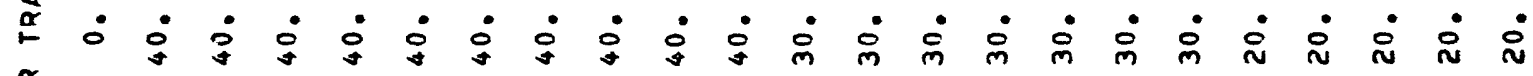
嵌 $\dot{0} \dot{0} \dot{0} \dot{0} \dot{0} \dot{0} \dot{0} \dot{0} \dot{\bar{m}} \dot{m} \dot{m} \dot{i} \dot{i} \dot{i} \dot{i} \dot{i}$ 道 $\dot{0} \dot{0} \dot{0} \dot{0} \dot{0} \dot{0} \dot{0} \dot{0} \dot{0} \dot{m} \dot{m} \dot{i} \dot{i} \dot{i} \dot{i}$

岸 $\dot{0} \dot{0} \dot{0} \dot{0} \dot{0} \dot{0} \dot{0} \dot{0} \dot{0} \dot{0} \dot{0} \dot{0} \dot{0} \dot{0} \dot{0} \dot{0} \dot{0}$ 
$\dot{0} \dot{0} \dot{0} \dot{0} \dot{0} \dot{0} \dot{0} \dot{0}$

$\dot{\circ} \dot{\circ} \dot{\circ} \dot{\circ} \dot{0}$

$\dot{\circ} \dot{\circ} \dot{\circ} \dot{\circ} \dot{\circ} \dot{\circ}$

$\dot{\square} \dot{\circ} \dot{\circ} \dot{\circ} \dot{\circ} \dot{0}$

$\dot{\circ} \dot{\circ} \dot{\circ} \dot{\circ} \dot{0} \dot{0}$

$\dot{\circ} \dot{\circ} \dot{\circ} \dot{\circ} \dot{0} \dot{0}$

$\dot{\circ} \dot{\vdots} \dot{\circ} \dot{\circ} \dot{0} \dot{0}$

$\dot{\circ} \dot{\circ} \dot{\circ} \dot{\circ} \dot{\circ} \dot{\circ}$

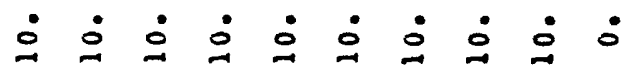

$\dot{\circ} \dot{\therefore} \dot{0} \dot{0} \dot{0} \dot{0}$

$\dot{\circ} \dot{\circ} \dot{0} \dot{\circ} \dot{0} \dot{0}$

$\dot{\circ} \dot{\therefore} \dot{0} \dot{0} \dot{0} \dot{0}$

$\dot{\circ} \dot{\circ} \dot{\circ} \dot{\circ} \dot{0} \dot{0}$

$\dot{\circ} \dot{\circ} \dot{\circ} \dot{\circ} \dot{0} \dot{0}$

$\dot{\circ} \dot{\circ} \dot{0} \dot{0} \dot{0} \dot{0}$

$\dot{\circ} \dot{\circ} \dot{0} \dot{0} \dot{0} \dot{0}$

$\dot{\circ} \dot{\circ} \dot{0} \dot{0} \dot{0} \dot{0}$

$\dot{\square} \dot{\square} \dot{0} \dot{0} \dot{0} \dot{0}$

$\dot{\circ} \dot{\circ} \dot{\circ} \dot{\circ} \dot{\circ} \dot{0}$

$\dot{\therefore} \dot{0} \dot{0} \dot{0} \dot{0} \dot{0}$

$\dot{\therefore} \dot{\therefore} \dot{0} \dot{0} \dot{0} \dot{0}$

$\dot{\circ} \dot{\circ} \dot{0} \dot{0} \dot{0} \dot{0}$

$\dot{\therefore} \dot{\therefore} \dot{0} \dot{0} \dot{0} \dot{0}$

$\dot{\circ} \dot{\therefore} \dot{0} \dot{0} \dot{0} \dot{0}$

$\dot{0} \dot{0} \dot{0} \dot{0} \dot{0} \dot{0} \dot{0}$

$\dot{0} \dot{0} \dot{0} \dot{0} \dot{0} \dot{0} \dot{0}$

$\dot{0} \dot{0} \dot{0} \dot{0} \dot{0} \dot{0} \dot{0}$

: $\dot{0} \dot{0} \dot{0} \dot{0} \dot{0}:$

$\therefore \dot{0} \dot{0} \dot{0} \dot{0} \dot{0}:$ 
000000000000000000000000000000000

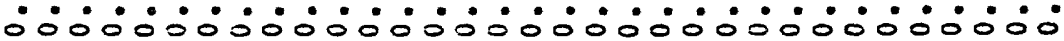
000000000000000000000000000000000

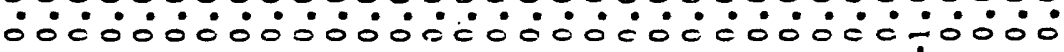
000000000000000000000000000000000

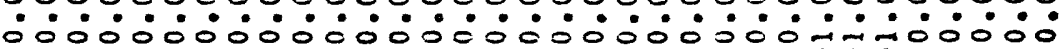
$00000000000000000000000000^{\prime} b_{0} 0000$ 000000000000000000000000000000000 -

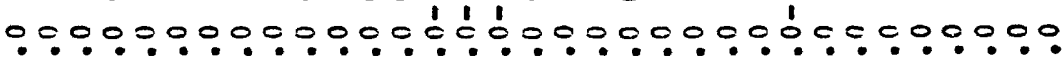

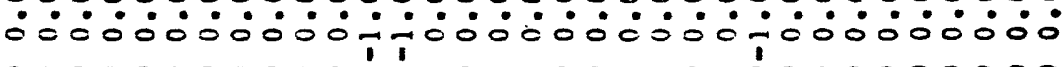
000000000000100000000000000000000

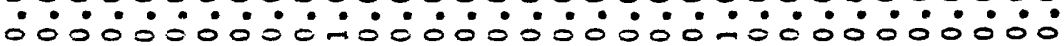
000000000070000000000010000000000
000000000000000000000000000000000 :

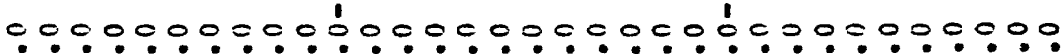
¿்́ $\dot{0} \dot{0} \dot{0} \dot{0} \dot{0} \dot{0} \dot{0} \dot{0} \dot{0} \dot{0} \dot{0} \dot{0}=\dot{0} \dot{0} \dot{0} \dot{0} \dot{0} \dot{0} \dot{0}$ 000000000000000000000000000000000

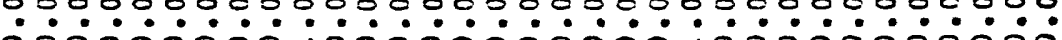

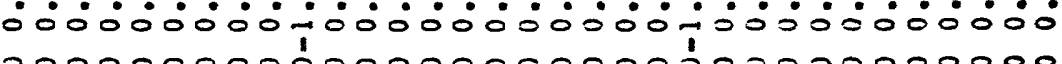
000000000000000000000100000000000

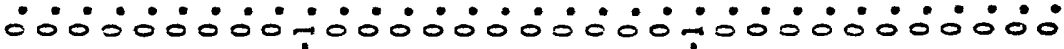
000000000700000000000700000000000

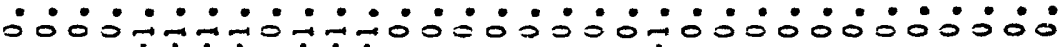
$0000 \overrightarrow{1} \overrightarrow{1} \dot{1} \overrightarrow{1} \overrightarrow{1} 00=00000 \overrightarrow{0} 00000000000$

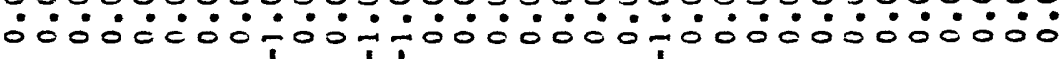
000000001000100000000000000000000 í: í: 00000000000000000010000000000000

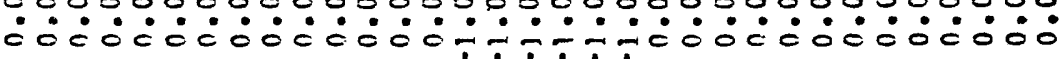

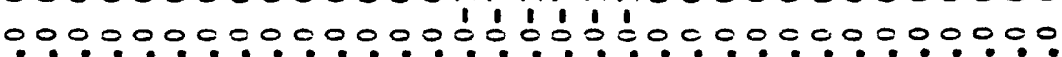

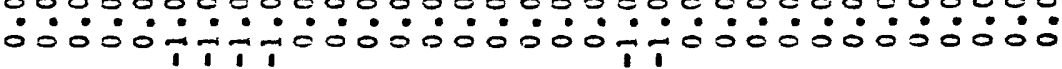

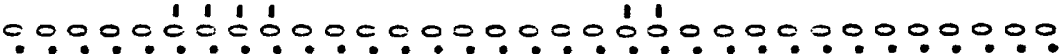
$\dot{0} \dot{0} \dot{0} \dot{0} \dot{0} \dot{0} \dot{1} \dot{1} \dot{0} \dot{0} \dot{0} \dot{0} \dot{0} \dot{0} \dot{0} \dot{0} \dot{0} \dot{0} \dot{0} \dot{0} \dot{0}$

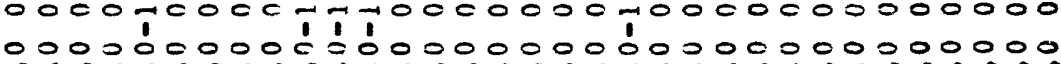
:0ं $000 \frac{1}{1} 000000-1000000011000000000000$ $000000000=0000000000000 \% 000000000$

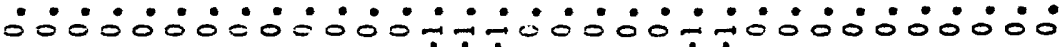

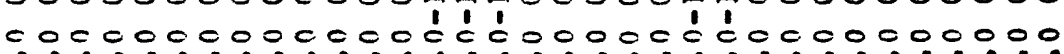
ல் $\dot{0} \dot{0} \dot{0} \dot{0} \dot{0} \dot{0} \dot{0} \dot{0} \dot{0} \dot{0} \dot{0} \dot{0} \dot{0} \dot{0} \dot{1} \dot{0} \dot{0} \dot{0} \dot{0} \dot{0}$ $00000=00=00000100000000 b^{\prime} b_{0} 000000$

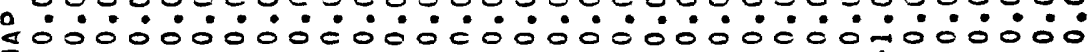
$\$ 000000000000000000000000007000000$

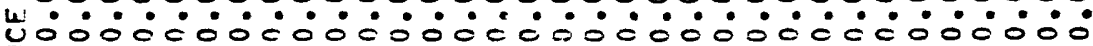
省o0000000000000000000000000000000

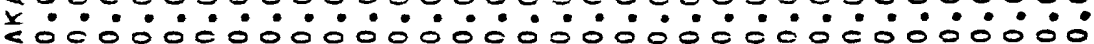
$\psi=0=0,000000000=0=0 c=0000000000000$ ब W000000000000000000000000000000000

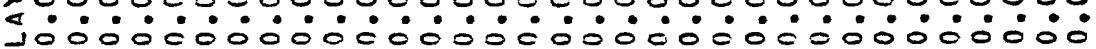
0000000000000000000000000000000000

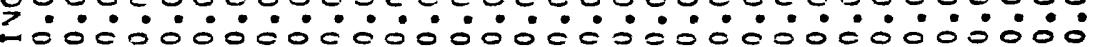

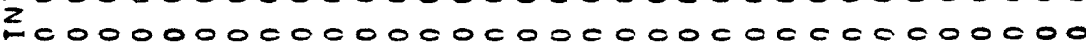
ش́: S000000000000000000000000000000000

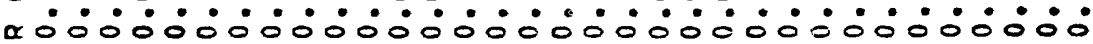
$\alpha 000000000000000000000000000000000$
$\omega 000000000000000000000000000000000$

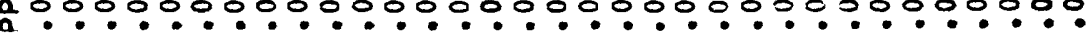


000000000000000000000000000000000

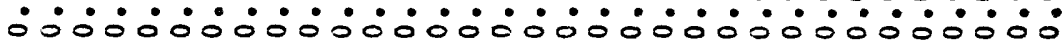
000000000000000000000000000000000

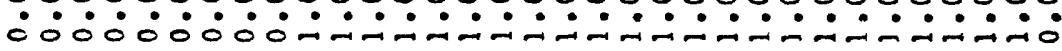
000000000000000000000000000000000

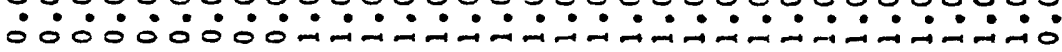
000000000000000000000000000000000

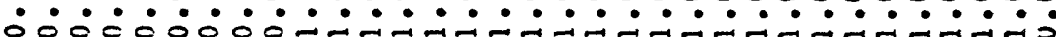
000000000000000000000000000000000

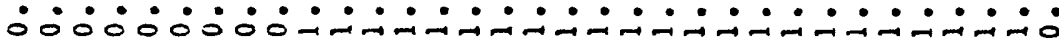
000000000000000000000000000000000

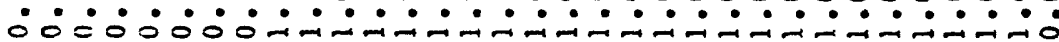
000000000000000000000000000000000

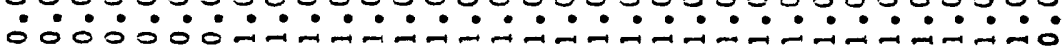
$000=00000000000000000000000000000$

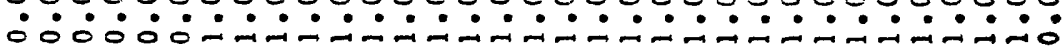
$00000=000000000000000000000000000$

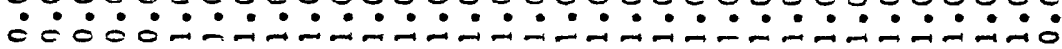
000000000000000000000000000000000

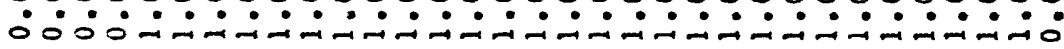
000000000000000000000000000000000

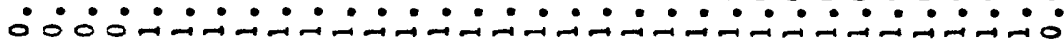
000000000000000000000000000000000 í: 000000000000000000000000000000000

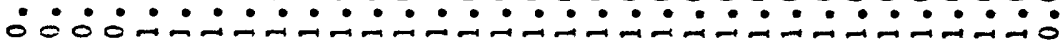
$000000000=00000000000000000000000$

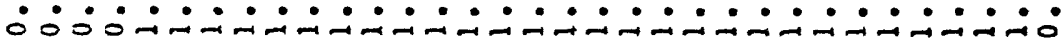
000000000000000000000000000000000

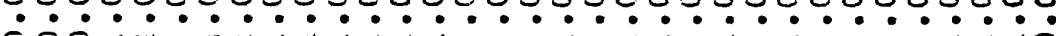
000000000000000000000000000000000

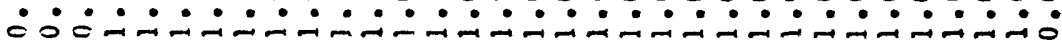
000000000000000000000000000000000

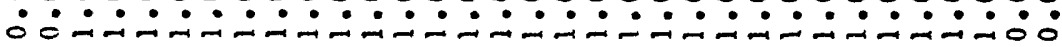
$000000=00000000000000000000000000$

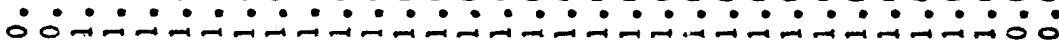
000000000000000000000000000000000

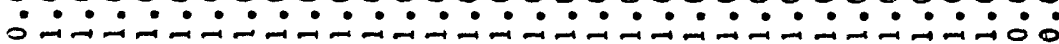
$0000000000000000000000=0000000000$

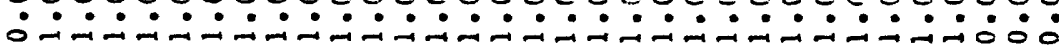
000000000000000000000000000000000

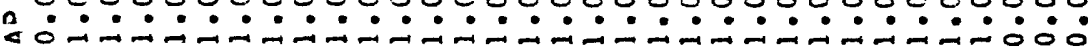
I000000000000000000000000000000000

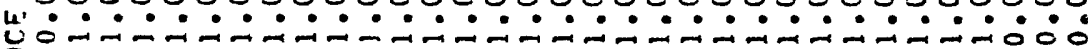
ব000000000000000000000000000000000

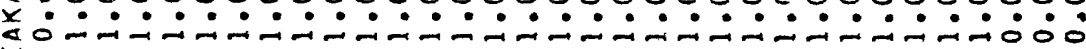
Lo00000000000000000000000000000000

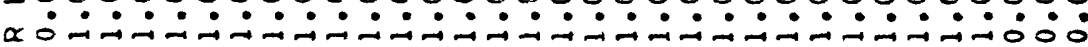
w000000000000000000000000000000000

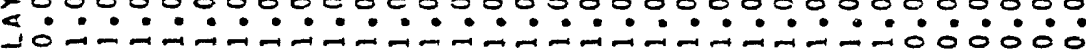
0000000000000000000000000000000000

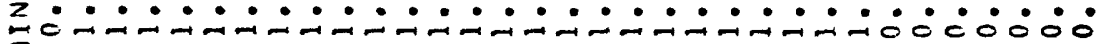
mo00000000000000000000000000000000 ये: U0:0000000000000000000000000000000

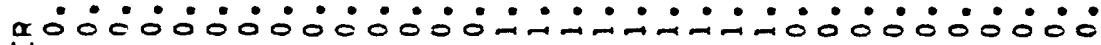
w000000000000000000000000000000000 o: 


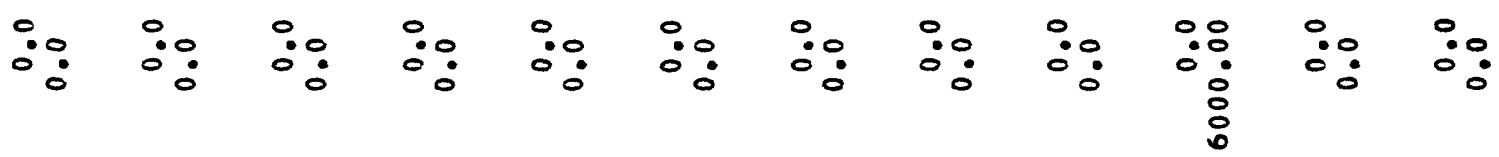

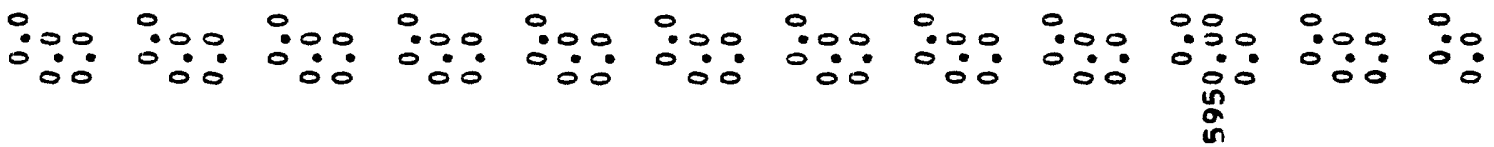

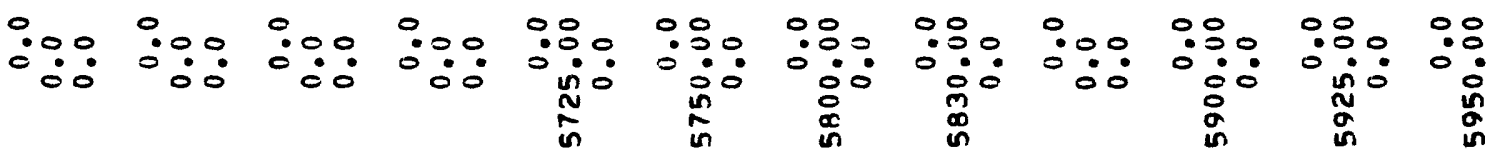

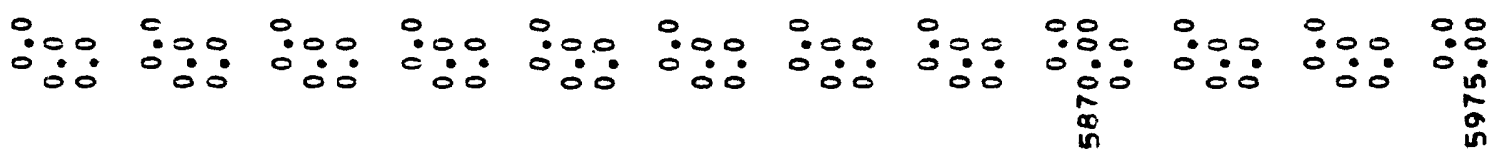

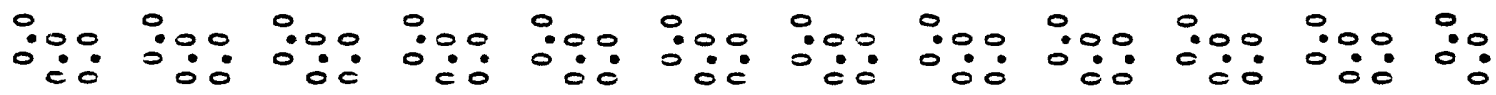

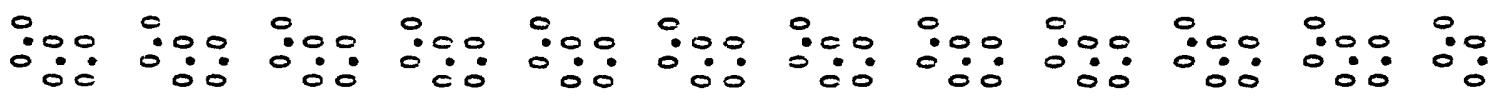

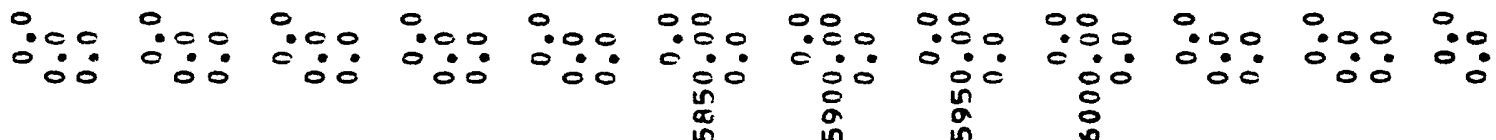

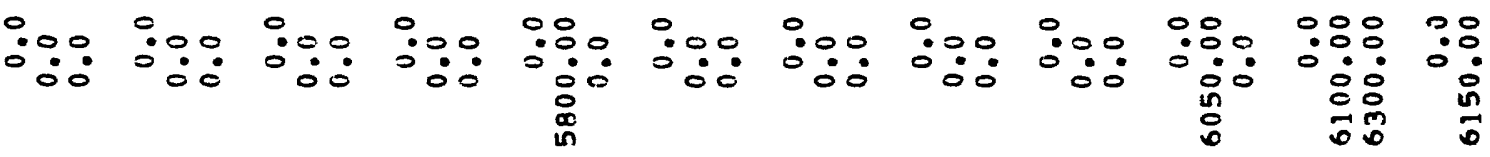

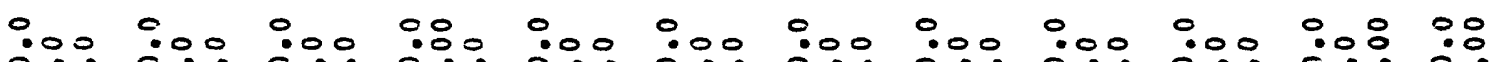

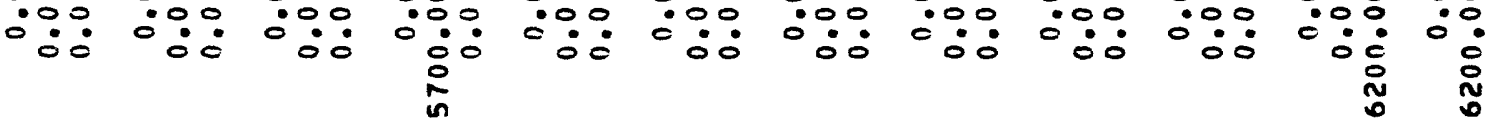

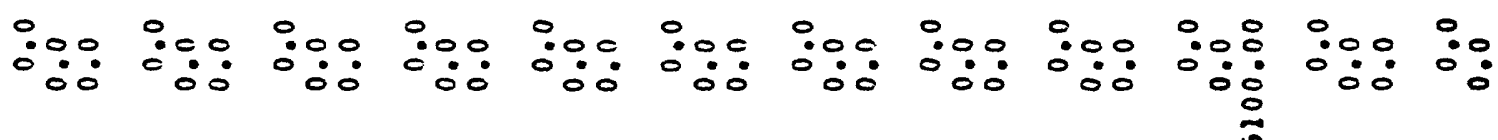




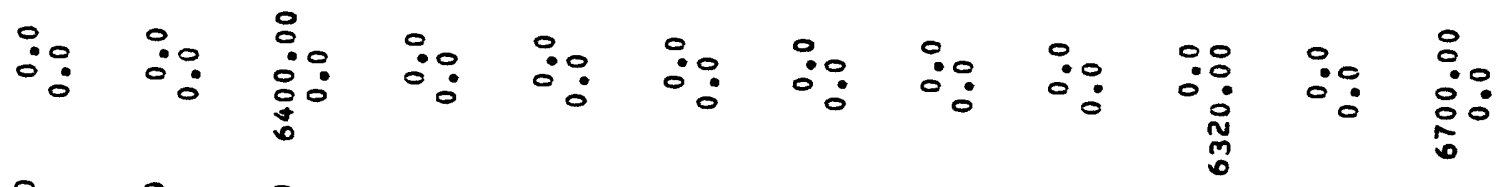

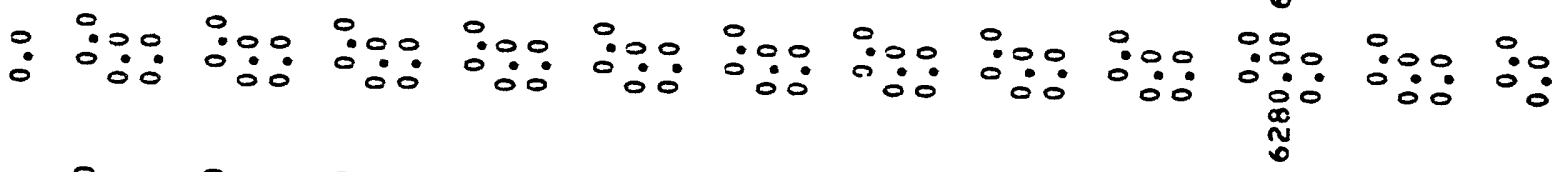

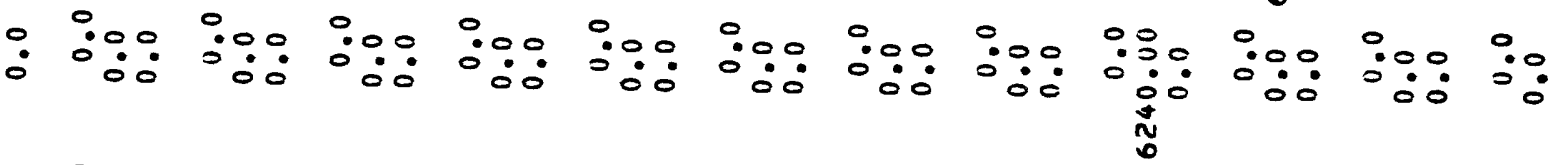

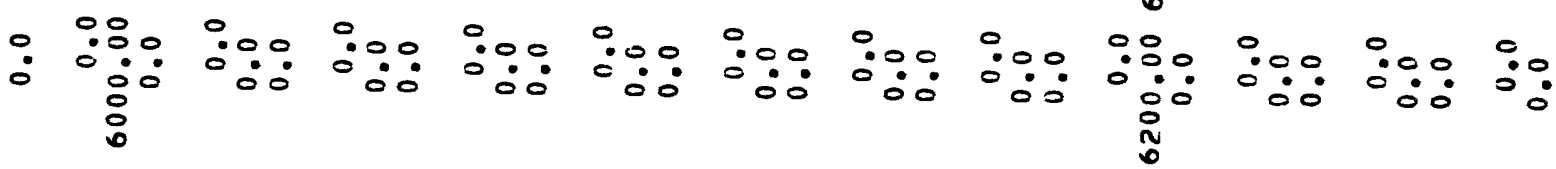
$\because \quad: 00$ :

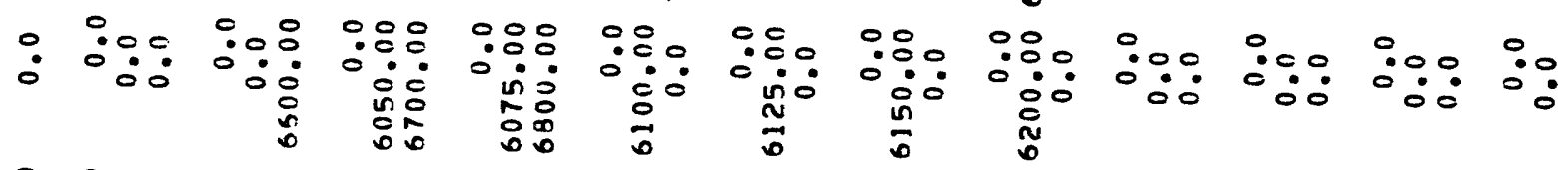

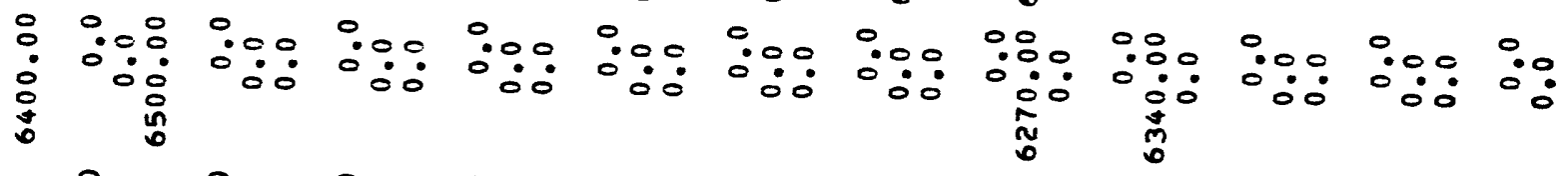

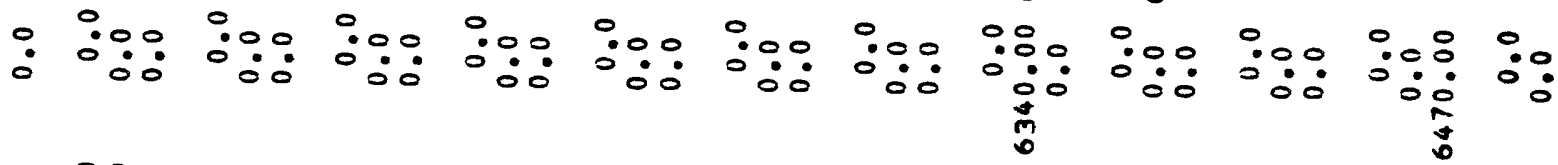
$\because$ :

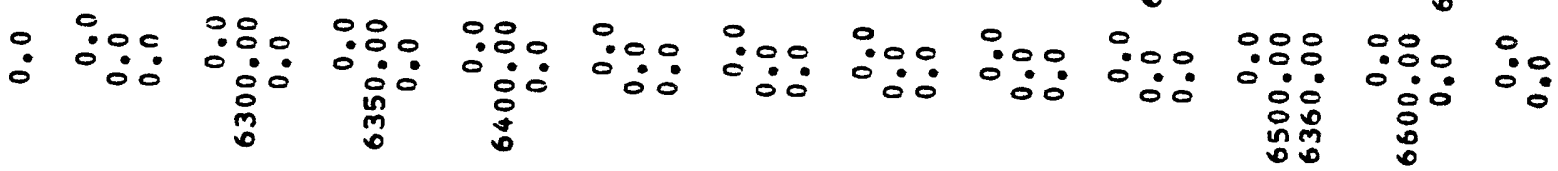




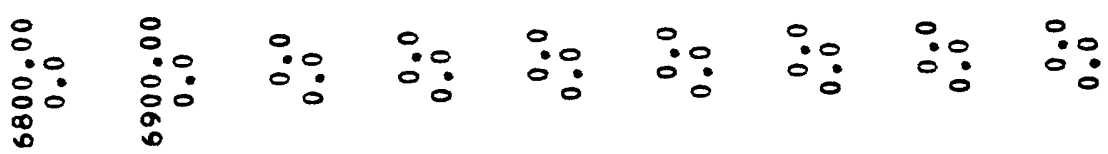

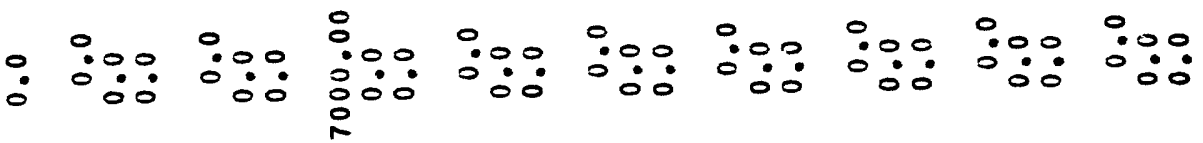

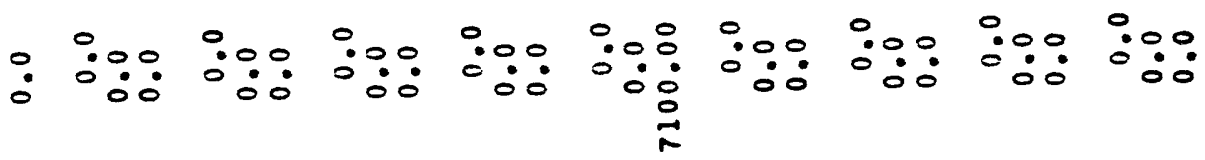

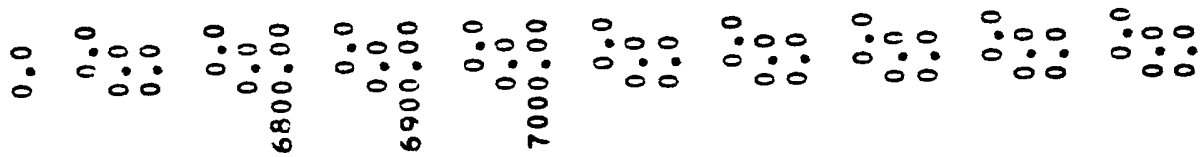

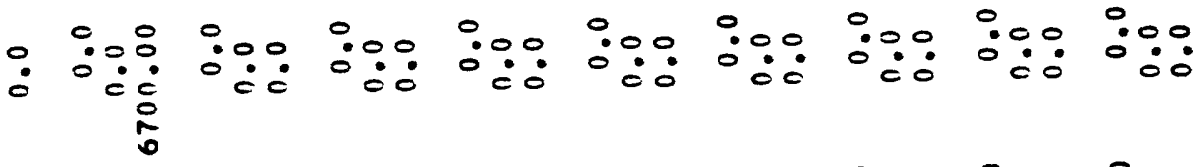

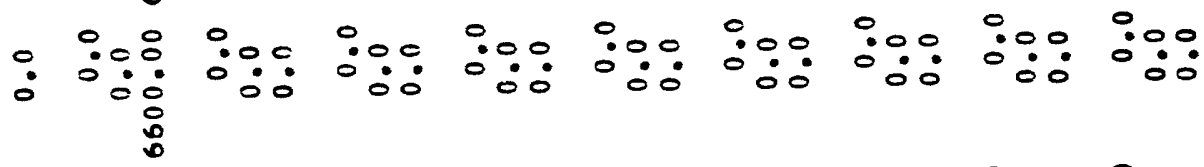

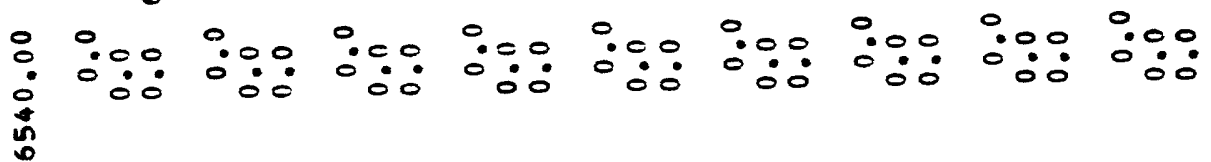

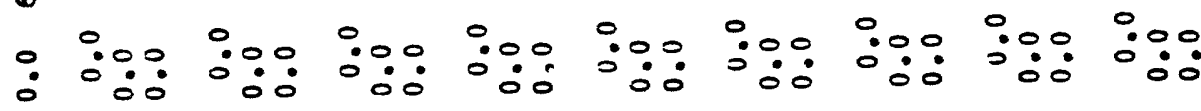

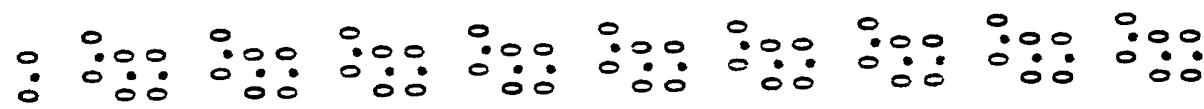

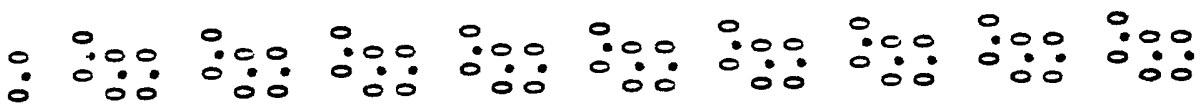




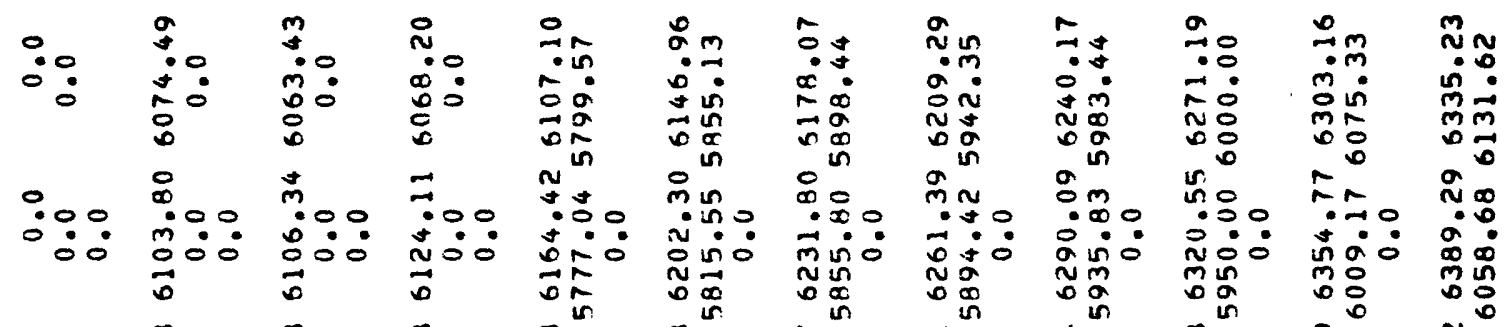

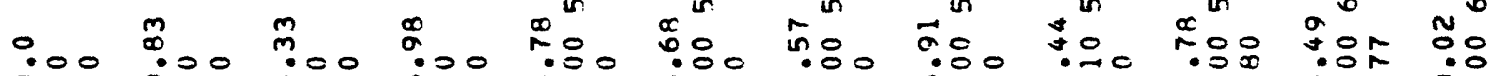

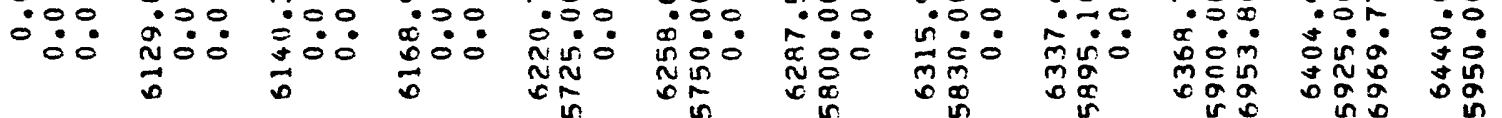

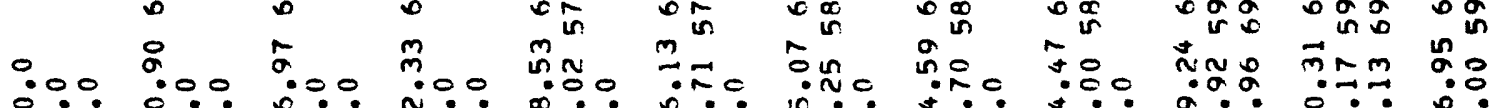

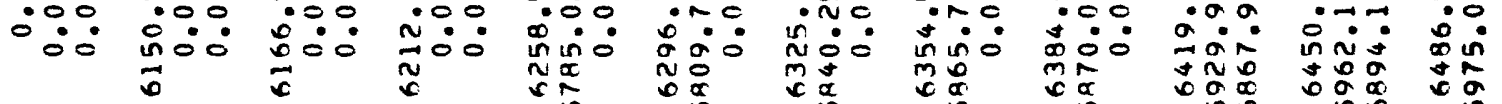

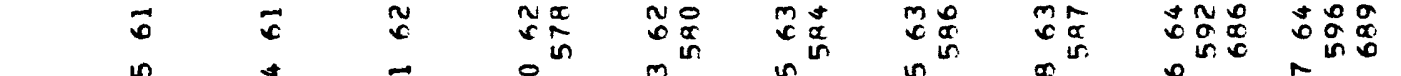

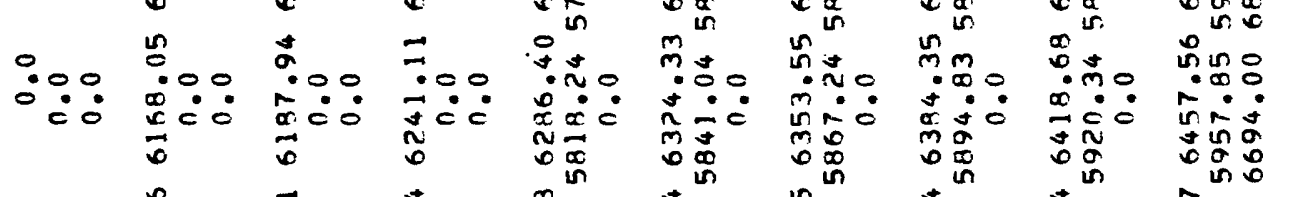

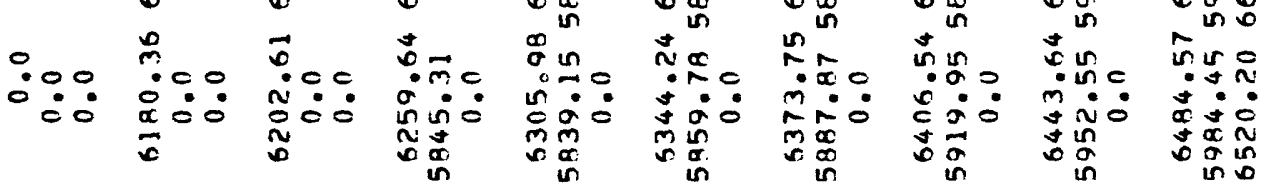

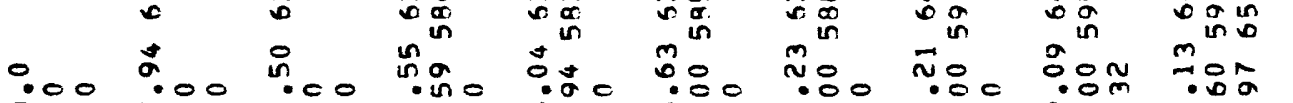

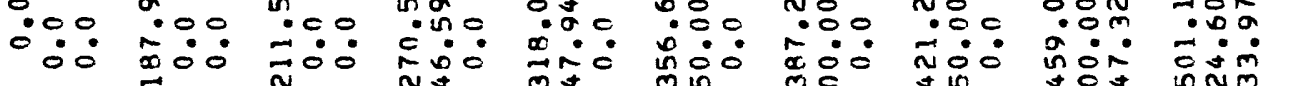
$\frac{\infty}{6} \quad \overrightarrow{\tilde{v}}$

究点

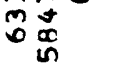
至怘

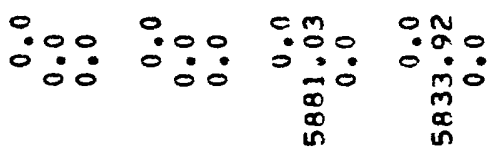
$\because 00$ 0.0 \%⿺ 菌览 $\therefore: \frac{0}{0}$

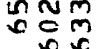
$\because$ 范 $\ddot{a} \dot{m}$ 高幺 in 0 of on å in士 ○。 $\sim$ n แn 0

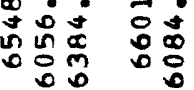

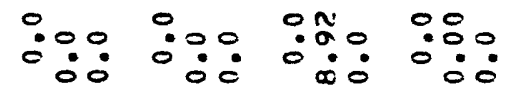

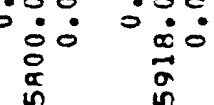

0 i。

020

$0 x$

$0: m$

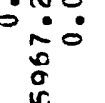

음

0
0
0
0
0

:

$\because \therefore 8$

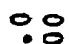

:0

둥

in

ox or

웅으.

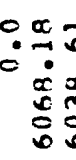

0.0

000

웡으

:웅

$\begin{array}{ll}\infty & 0 \\ 0 & 0 \\ \text { in } & 0\end{array}$

io $\frac{a}{a}$

官品

둥

0

mi:

0 :

$\stackrel{0}{0}$

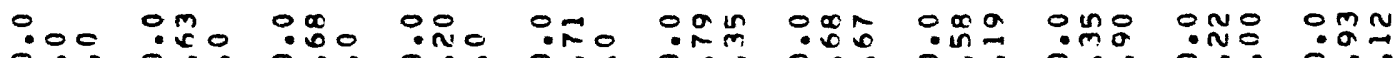

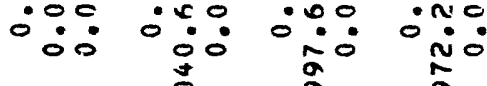
要

in

in

ํㅜㅇ

ia

용

둥

0

:m

吉吉

$: \div 0$
0
0
0
0

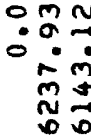

$0: 0$
$0: 0$
0
0
0 


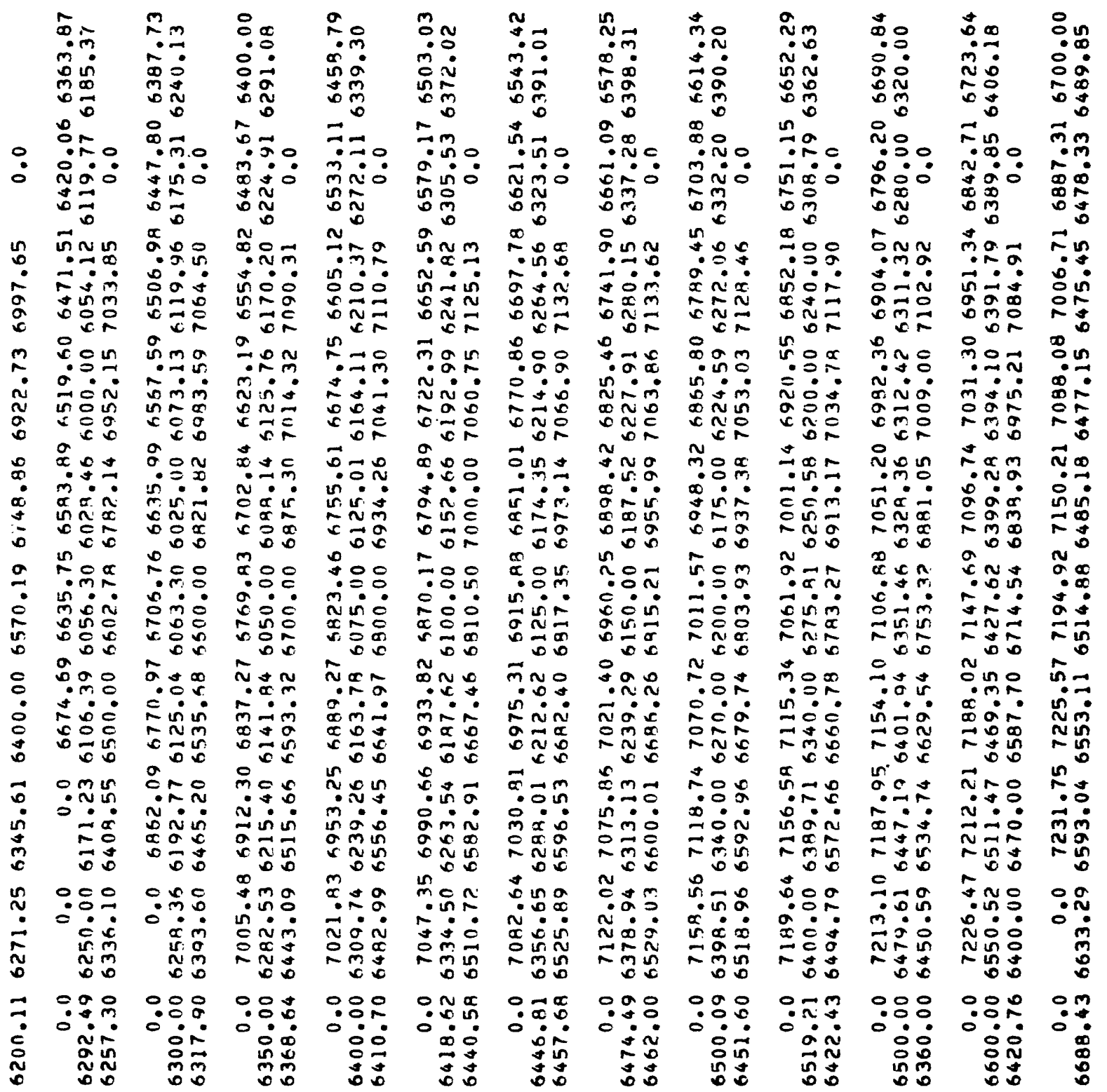




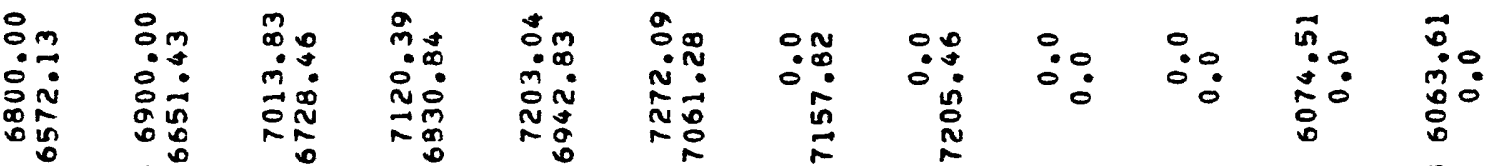
ํ. $m^{0}$ :

5o. तี่ं: ूํㅇㅇ oु 5
0.0 ถึกน แ $\pm \rightarrow n$ 웅 - 0 in กำ $\infty$ $\rightarrow 0^{\circ}$ जี⿻:日 㲾员 $\operatorname{nin} a+\infty$

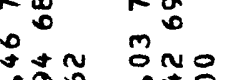

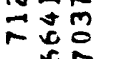

- $\div: 0$. iे: iñ $\operatorname{tac}$

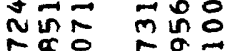
O0: 동

人类

$\because \div$ $x: \dot{0}$

$\Delta \div$ $\rightarrow$ in 0 $\because \propto 0$ g. $x$ $\infty=N$ ñ.0 nin:

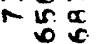
mino

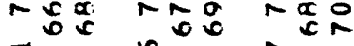
mo음

mo:

ro용 ต농요 울 $\because \stackrel{\infty}{N}$ o.

ana

웅

$\forall$

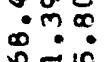

m̃n

0

n

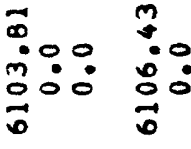

केष:

N舟足

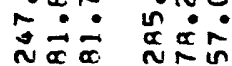

$\sim \alpha \propto$

Nin

$\infty \infty$

a $\dot{m}$

in

$m \pi m$

$\sim \infty$

in 0

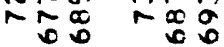

뭉ㅇㅇ

ini:

Tñ

$\sim 00$

oma

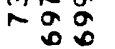

0 每

$\because 00$ :

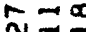

Nơ

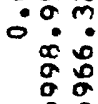

-

$\because: 0$

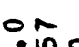

0000

?ำ

$\therefore \therefore$.

동

요

$n_{\infty}^{n} N$

$\div 0 \infty$

70

웅

C

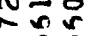

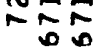

Imin

un

$0 \rightarrow$ in

- $-n$

$\begin{array}{ll}0 & 0 \\ 0 & 0\end{array}$

$\sum_{i=0}^{0}$

- m

$\therefore$.

$m \infty \%$

$\infty$ i

Nin

$\dot{0}$

00

N

No

60

ẫ

$m \vec{c}$

a

- :-

$\stackrel{n}{=}$

0 -

$\therefore: 0$

$\underset{0}{0} 0$

$0 \div 0$

0 하

으음

$0 \div$

$0: \dot{0}$

i i

ํํㅇ

0 in

00

in

on

mi்

$\begin{array}{lll}\infty & 1 & 0 \\ 0 & 0 & 0 \\ 0 & 0\end{array}$

ç弋

om 0

ono

$\therefore 0$

0 in

$\pm \frac{1}{2}$

$\because \pi N$

ㅁ․ 0 :

- 요 in 옷

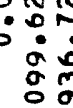

oc n

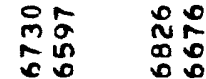

$\dot{0} \mathbf{0} \dot{0}$

a

웅

$\underset{\infty}{\infty}$

O 0 Ont O\& O ON

00

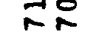

1

$\because 0 \pi$

茴: $0: 00: 0$

r

$0 \infty a$

00

$\infty_{000}$

요

$0 \div \dot{0}$

N

$\therefore: 00: 00$

ก :

0

: -0

CN

-00 000

$\therefore \infty$

$c \infty$

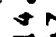

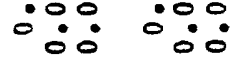

N $N$

oํำ

0.0

in

$\vec{n}$

onn

:

$\therefore: 0$

in

$\vec{n}$

$0 \leq n$

in

20

$0 \times$

: 뜸

满

$\sim N$

$00 \rightarrow$

0 in

กิำ

$N$

옴

0 No

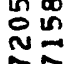

$\because 0 \%$

$\because 00: 00:$

$\because 00: 000$

$0000 \stackrel{0}{0} 00^{n}$

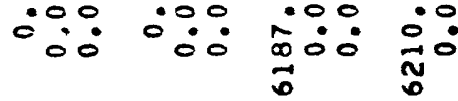

:00 :00 0.00 난

0:0 0:00 0:0

in

$0 \div \%$

0.0

0

0

$\stackrel{m}{N}$

$0:$

$0: 0$

$\because 00$

$: 00$

$\because 0$

o n $\infty$

on

$0 \div$ :

No

잉

$\circ \%$

$\circ$.

足

$\because \div$

品品

$\stackrel{n}{N}$

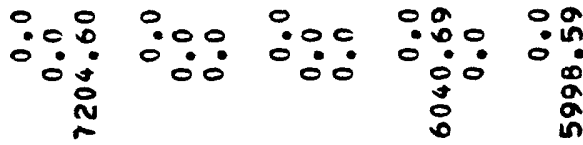




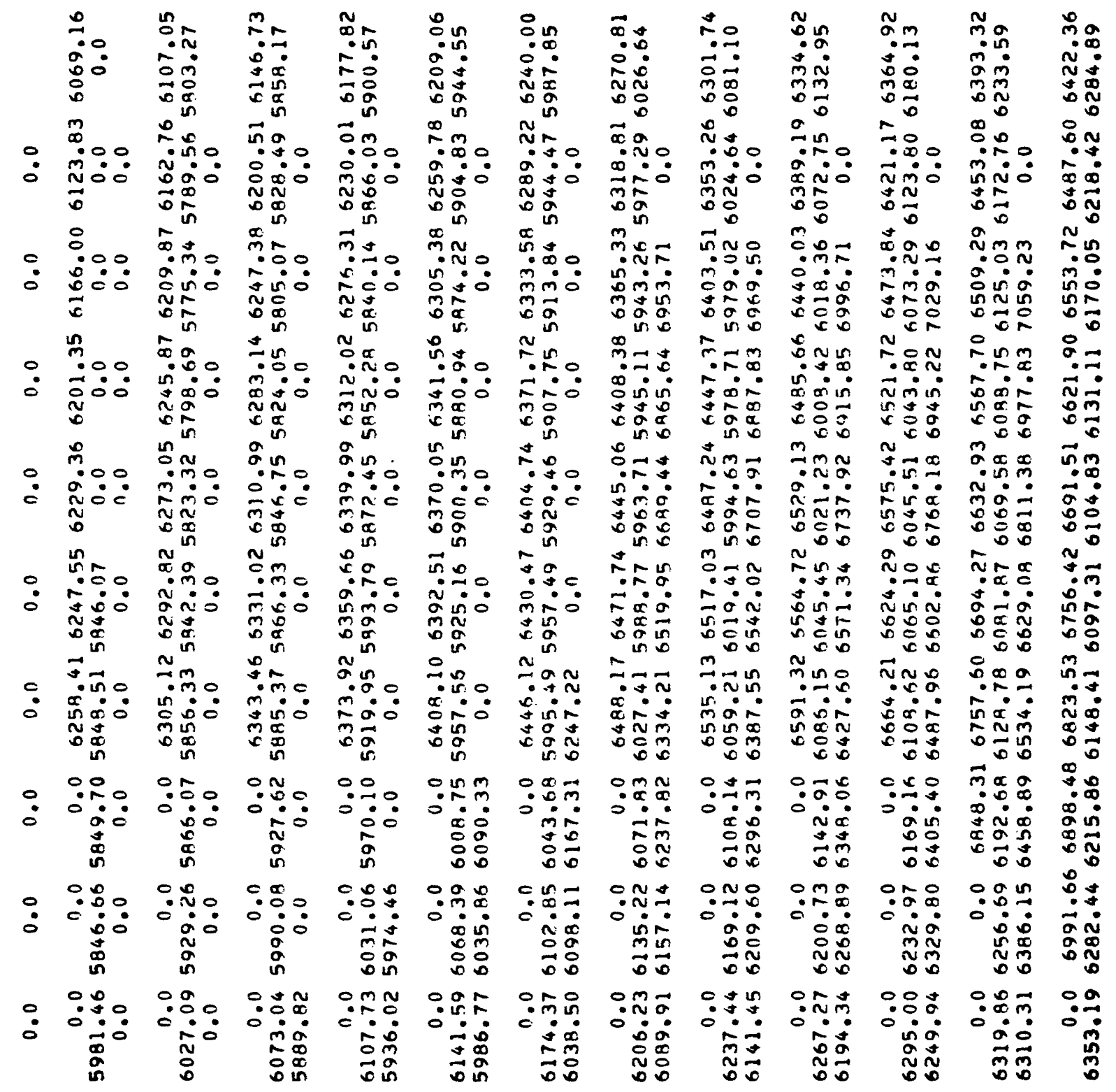




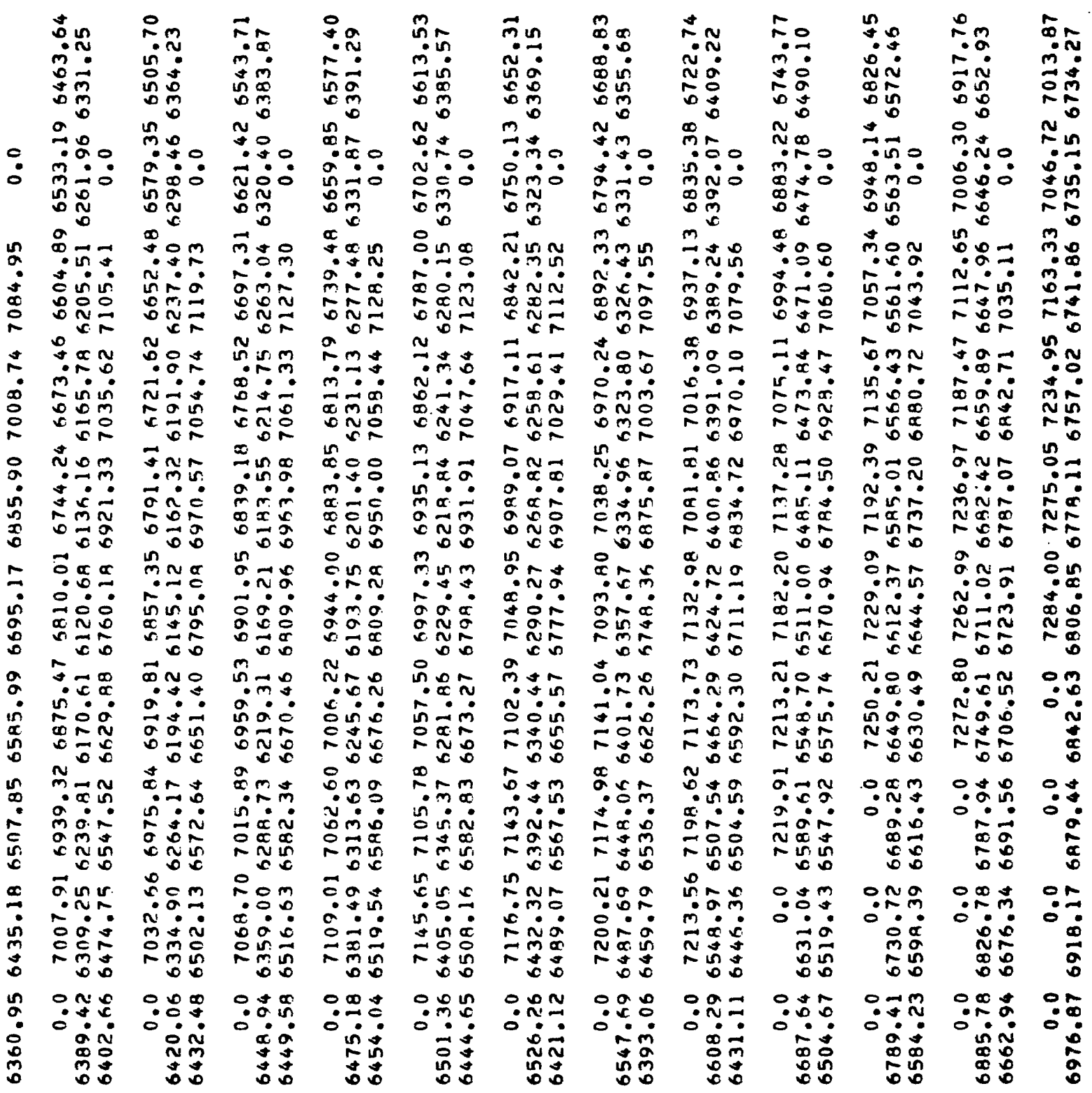




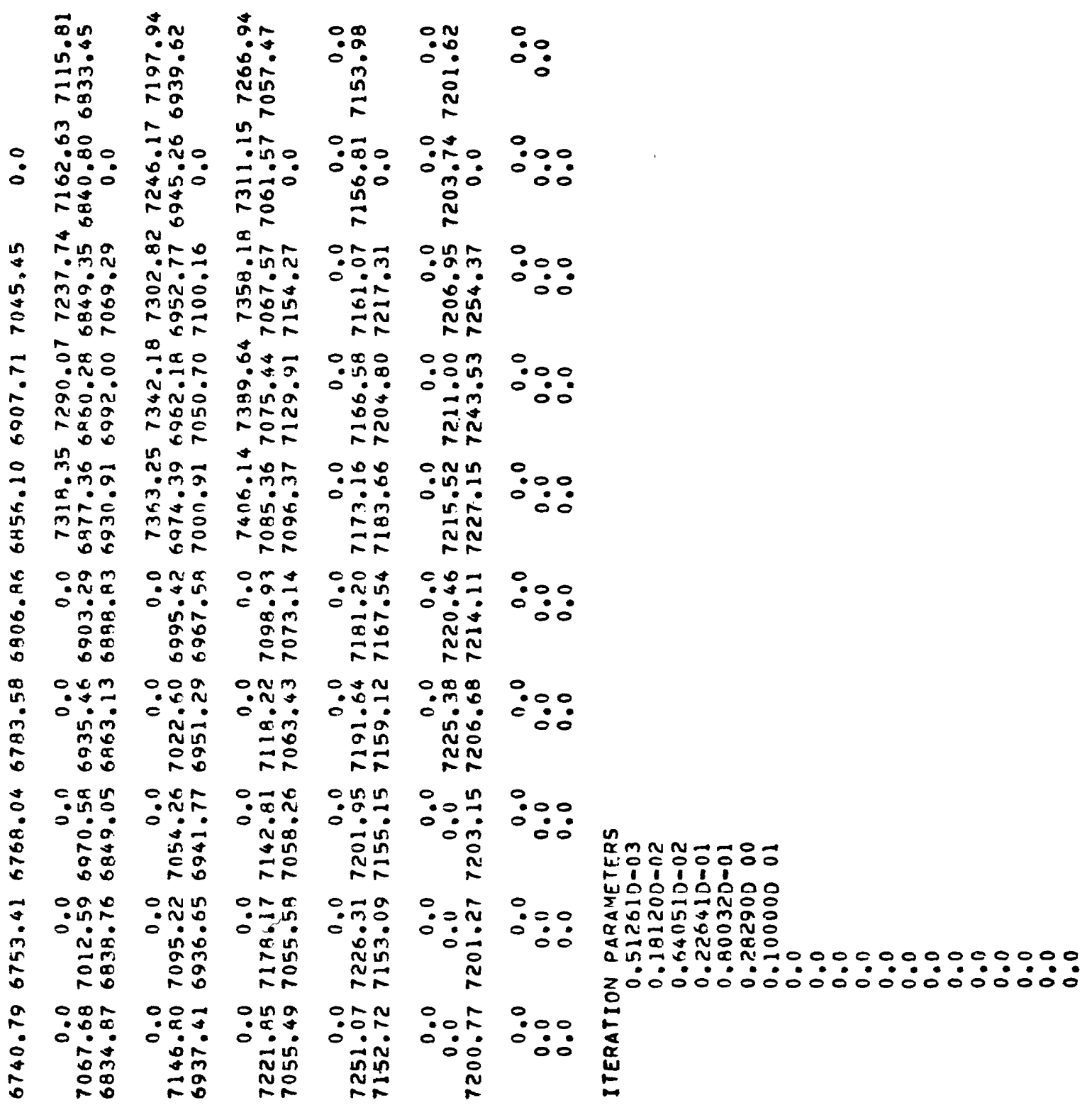


$\dot{0} \dot{0} \dot{0} \dot{0} \dot{0} \dot{0} \dot{0} \dot{0} \dot{0} \dot{0} \dot{0} \dot{0} \dot{0} \dot{0} \dot{0}$ $\dot{0} \dot{0} \dot{0} \dot{0} \dot{0} \dot{0} \dot{0} \dot{0} \dot{m} \dot{m} \dot{m} \dot{m} \dot{m} \dot{m} \dot{m} \dot{m} \dot{m} \dot{m}$ $\dot{0} \dot{0} \dot{0} \dot{0} \dot{0} \dot{0} \dot{0} \dot{m} \dot{m} \dot{m} \dot{m} \dot{m} \dot{m} \dot{m} \dot{m} \dot{m} \dot{m} \dot{m} \dot{m} \dot{m}$ $\dot{0} \dot{0} \dot{0} \dot{0} \dot{0} \dot{0} \dot{0} \dot{0} \dot{m} \dot{m} \dot{m} \dot{m} \dot{m} \dot{m} \dot{m} \dot{m} \dot{m} \dot{m} \dot{m} \dot{m}$ $\dot{0} \dot{0} \dot{0} \dot{0} \dot{0} \dot{0} \dot{0} \dot{0} \dot{m} \dot{0} \dot{m} \dot{m} \dot{m} \dot{m} \dot{m} \dot{m} \dot{m} \dot{m}$ $\dot{0} \dot{0} \dot{0} \dot{0} \dot{0} \dot{0} \dot{0} \dot{0} \dot{0} \dot{m} \dot{m} \dot{m} \dot{m} \dot{m} \dot{m} \dot{m} \dot{m}$ $\dot{0} \dot{0} \dot{0} \dot{0} \dot{0} \dot{0} \dot{0} \dot{0} \dot{0} \dot{0} \dot{m} \dot{m} \dot{m} \dot{m} \dot{m} \dot{m} \dot{m} \dot{m} \dot{m} \dot{0}$ $\dot{0} \dot{0} \dot{0} \dot{0} \dot{0} \dot{0} \dot{0} \dot{0} \dot{0} \dot{m} \dot{m} \dot{m} \dot{m} \dot{m} \dot{m} \dot{m} \dot{m} \dot{0} \dot{0}$ 岕 $\dot{L}: \dot{0} \dot{0} \dot{0} \dot{0} \dot{0} \dot{0} \dot{0} \dot{m} \dot{m} \dot{m} \dot{m} \dot{m} \dot{m} \dot{m} \dot{0}$ $\dot{0} \dot{0} \dot{0} \dot{0} \dot{0} \dot{0} \dot{0} \dot{0} \dot{0} \dot{0} \dot{0} \dot{0} \dot{m} \dot{m} \dot{m} \dot{m} \dot{m} \dot{0} \dot{0}$ $\dot{0} \dot{0} \dot{0} \dot{0} \dot{0} \dot{0} \dot{0} \dot{0} \dot{0} \dot{0} \dot{m} \dot{m} \dot{0} \dot{m} \dot{m} \dot{0} \dot{0}$ $\dot{0} \dot{0} \dot{0} \dot{0} \dot{0} \dot{0} \dot{0} \dot{0} \dot{0} \dot{0} \dot{0} \dot{0} \dot{0} \dot{0} \dot{0} \dot{0}$ $\dot{0} \dot{0} \dot{0} \dot{0} \dot{0} \dot{0} \dot{0} \dot{0} \dot{0} \dot{0} \dot{0} \dot{0} \dot{0} \dot{0} \dot{0} \dot{0} \dot{0} \dot{0}$ 湈 $\dot{0} \dot{0} \dot{0} \dot{0} \dot{0} \dot{0} \dot{0} \dot{0} \dot{0} \dot{0} \dot{0} \dot{0} \dot{0} \dot{0} \dot{0} \dot{0} \dot{0} \dot{0}$ $\dot{0} \dot{0} \dot{0} \dot{0} \dot{0} \dot{0} \dot{0} \dot{0} \dot{0} \dot{0} \dot{0} \dot{0} \dot{0} \dot{0} \dot{0} \dot{0} \dot{0}$ $\dot{0} \dot{0} \dot{0} \dot{0} \dot{0} \dot{0} \dot{0} \dot{0} \dot{0} \dot{0} \dot{0} \dot{0} \dot{0} \dot{0} \dot{0}$ $\dot{0} \dot{0} \dot{0} \dot{0} \dot{0} \dot{0} \dot{0} \dot{0} \dot{0} \dot{0} \dot{0} \dot{0} \dot{0} \dot{0} \dot{0} \dot{0}$ $\dot{0} \dot{0} \dot{0} \dot{0} \dot{0} \dot{0} \dot{0} \dot{0} \dot{0} \dot{0} \dot{0} \dot{0} \dot{0} \dot{0} \dot{0} \dot{0} \dot{0}$ N $\dot{\widetilde{W}} \dot{0} \dot{0} \dot{0} \dot{0} \dot{0} \dot{0} \dot{0} \dot{0} \dot{0} \dot{0} \dot{0} \dot{0} \dot{0} \dot{0} \dot{0} \dot{0} \dot{0} \dot{0} \dot{0}$ $\dot{0} \dot{0} \dot{0} \dot{0} \dot{0} \dot{0} \dot{0} \dot{0} \dot{0} \dot{0} \dot{0} \dot{0} \dot{i} \dot{i}$ 这 $\dot{0} \dot{0} \dot{0} \dot{0} \dot{0} \dot{0} \dot{0} \dot{0} \dot{0} \dot{0} \dot{0} \dot{0} \dot{0} \dot{0} \dot{0} \dot{i}$

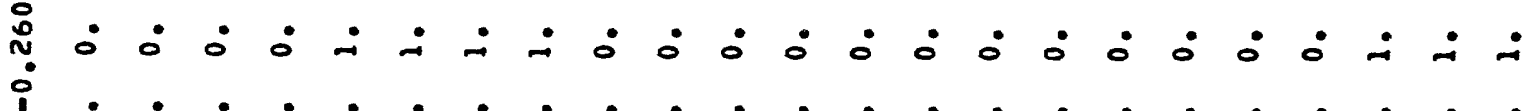
$\dot{0} \dot{0} \dot{\sim} \dot{\sim} \dot{\sim} \dot{\sim} \dot{0} \dot{0} \dot{0} \dot{0} \dot{0} \dot{0} \dot{0} \dot{ }$

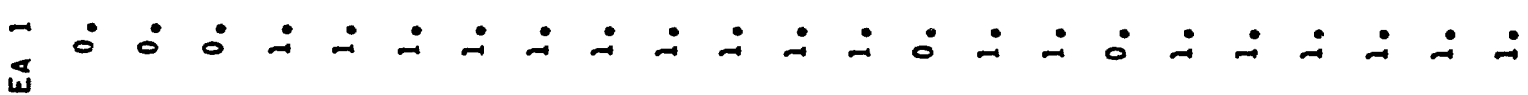

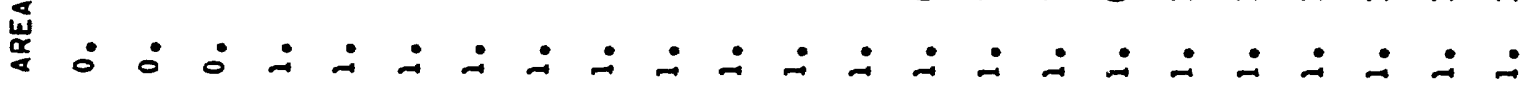

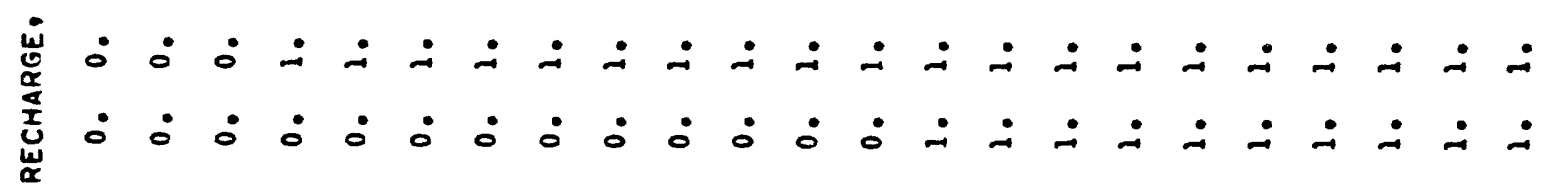

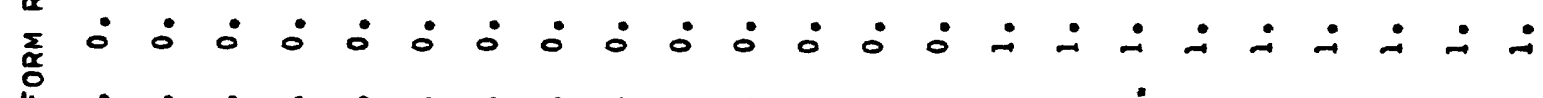

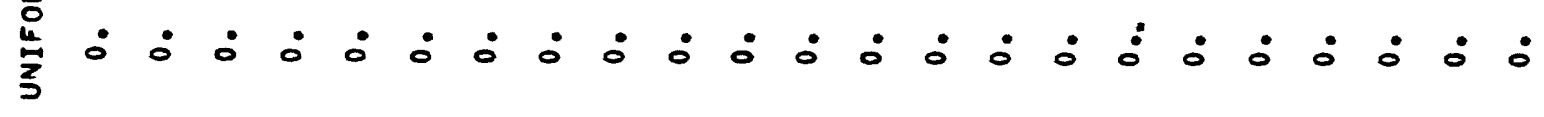

95 


$$
\begin{aligned}
& \therefore \dot{0} \dot{0} \dot{0} \dot{0} \dot{0} \\
& \dot{m} \dot{m} \dot{\sim} \dot{\sim} \dot{\sim} \dot{\sim} \dot{N} \dot{N} \dot{0} \\
& \dot{m} \dot{i} \dot{0} \dot{i} \dot{\sim} \dot{\sim} \dot{\sim} \\
& \dot{m} \dot{i} \dot{\sim} \dot{N} \dot{\sim} \dot{\sim} \dot{\sim} \dot{\sim} \dot{0} \\
& \dot{m} \dot{\sim} \dot{\sim} \dot{\sim} \dot{\sim} \dot{\sim} \dot{\sim} \dot{\sim} \\
& \dot{0} \dot{\sim} \dot{\sim} \dot{\sim} \dot{N} \dot{N} \dot{N} \dot{\sim}:
\end{aligned}
$$

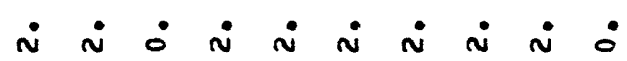

$$
\begin{aligned}
& \therefore \dot{0} \dot{0} \dot{i} \dot{N} \dot{N} \dot{0} \\
& \dot{0} \dot{0} \dot{0} \dot{\sim} \dot{\sim} \dot{\sim} \dot{\sim} \dot{\sim} \quad \dot{0} \\
& \therefore \dot{0} \dot{0} \dot{\sim} \dot{N} \dot{N} \dot{\sim} \\
& \therefore \dot{0} \dot{0} \dot{N} \dot{N} \dot{N} \dot{N} \dot{N} \\
& \dot{0} \dot{0} \dot{\sim} \dot{\sim} \dot{N} \dot{N} \dot{\sim} \\
& \therefore \dot{0} \dot{0} \dot{\sim} \dot{\sim} \dot{\sim} \dot{\sim} \dot{0} \\
& \therefore \dot{0} \dot{0} \dot{0} \dot{N} \dot{N} \dot{\sim} \\
& \therefore \dot{0} \dot{N} \dot{N} \dot{N} \dot{N} \dot{N} \dot{0} \\
& \dot{0} \dot{\sim} \dot{\sim} \dot{\sim} \dot{N} \dot{N} \dot{N}: \\
& \therefore \dot{\sim} \dot{\sim} \dot{N} \dot{N} \dot{N} \dot{\sim}: \dot{0} \\
& \dot{0} \dot{0} \dot{\sim} \dot{N} \dot{N} \dot{N} \dot{0} \\
& \dot{\Delta} \dot{\sim} \dot{\sim} \dot{\sim} \dot{\sim} \dot{\sim} \dot{\sim} \dot{0} \\
& \dot{0} \dot{0} \dot{\sim} \dot{\sim} \dot{\sim} \dot{0}: \dot{0} \\
& \dot{\sim} \dot{\sim} \dot{\sim} \dot{\sim} \dot{\sim} \dot{\sim} \dot{\sim} \dot{0} \dot{0} \\
& \therefore \dot{I} \dot{-} \dot{\dot{0}} \dot{0} \\
& \dot{I} \dot{I} \dot{a} \dot{0} \dot{0}
\end{aligned}
$$

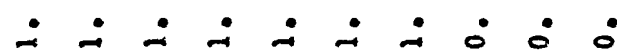

$$
\begin{aligned}
& \dot{I} \dot{i} \dot{0} \dot{0} \dot{0} \\
& \dot{i} \dot{i} \dot{0} \dot{0} \dot{0} \dot{0} \\
& \text { } \dot{0} \dot{0} \dot{0} \dot{0} \dot{0} \\
& \dot{0} \dot{0} \dot{0} \dot{0} \dot{0} \dot{0} \\
& \dot{0}: \dot{0}: \dot{0} \dot{0}: z^{\frac{\mathrm{W}}{z}}
\end{aligned}
$$




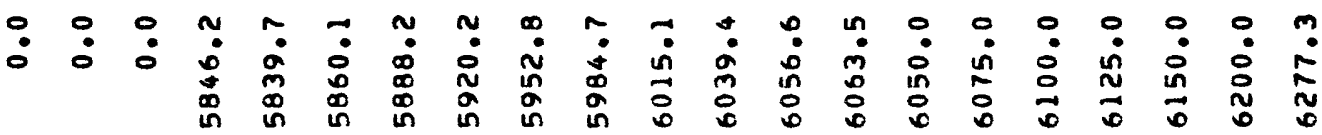

00000010000000000000000000 nomomoncNom00000

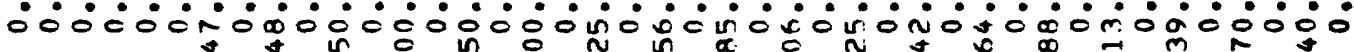

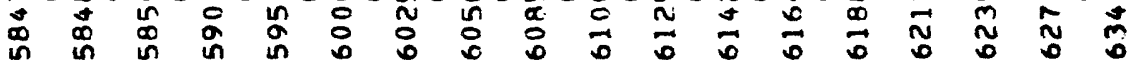

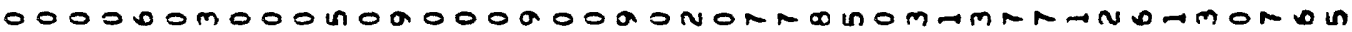
ட் $\dot{0} \dot{0} \dot{0} \dot{0} \dot{0} \dot{0} \dot{0} \dot{0} \dot{0} \dot{0} \dot{0} \dot{0} \dot{0} \dot{0} \dot{0} \dot{0} \dot{0} \dot{0} \dot{0} \dot{0} \dot{0} \dot{0} \dot{0} \dot{0} \dot{0}$

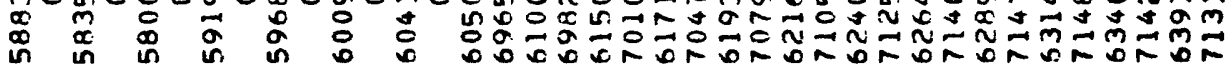

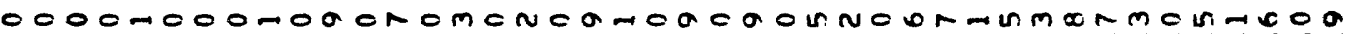

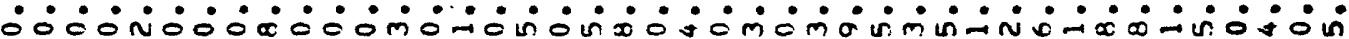

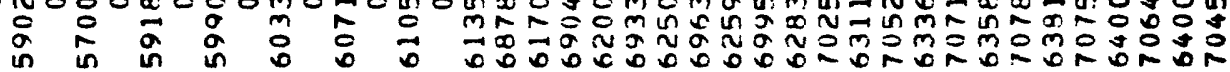

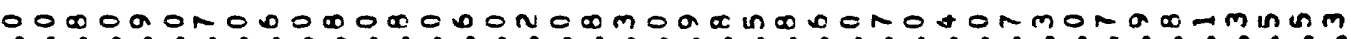

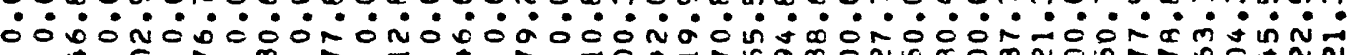
o a

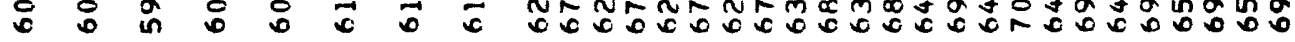

$0000 m 0000000 N 0$ tonoOLn

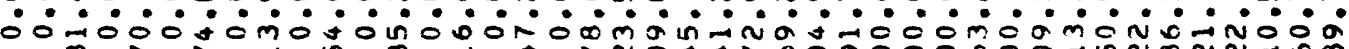

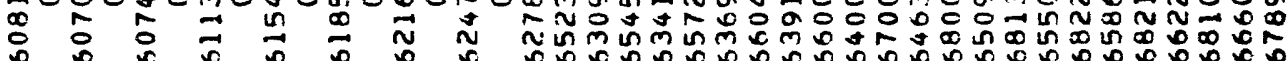

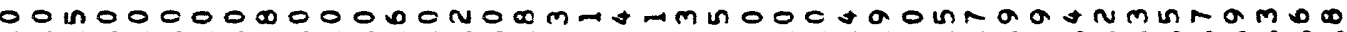

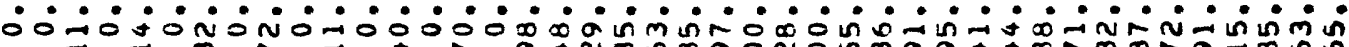

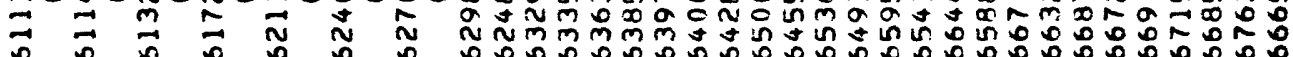

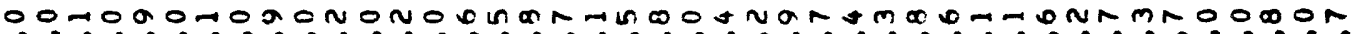

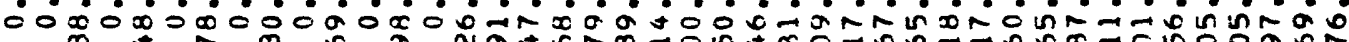

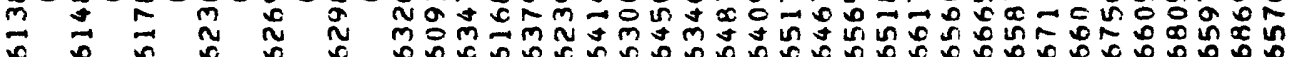

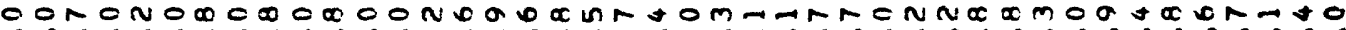

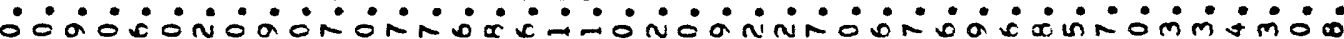
in

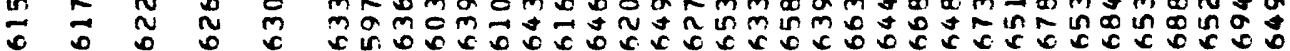

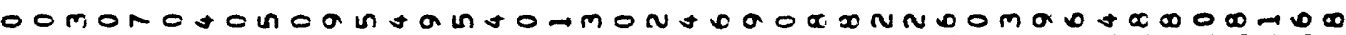

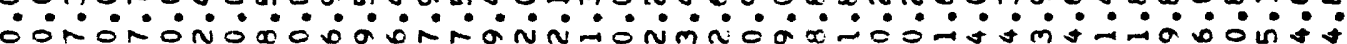
I a w o

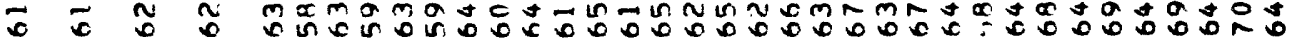

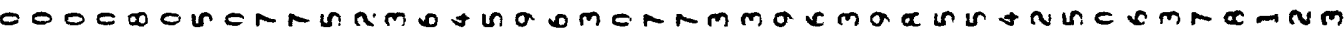

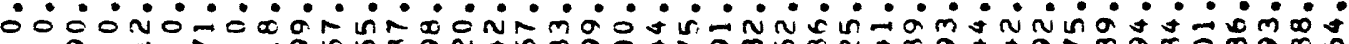

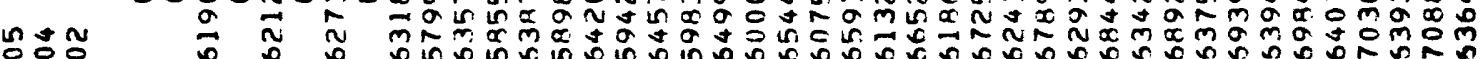

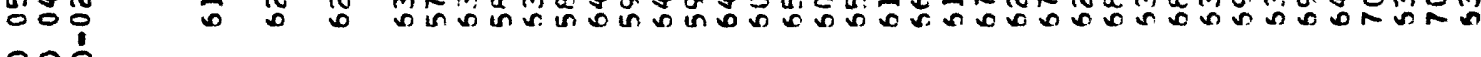
응응 $00 \mathrm{~m}$ $\forall \forall$ $\infty \sim N$

$\therefore$ :ं:

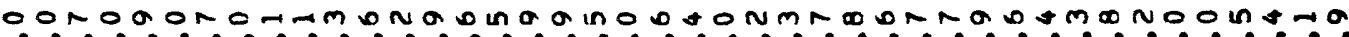

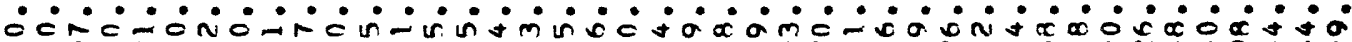

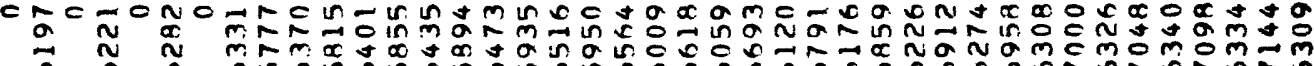

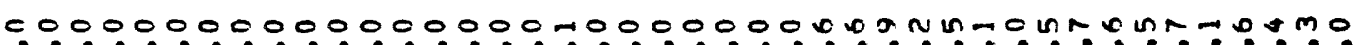
T⿱乛龰 $\|$ "

$\ln \bar{n} \bar{n}$

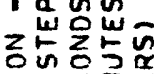

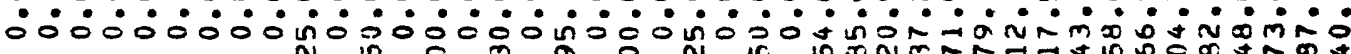

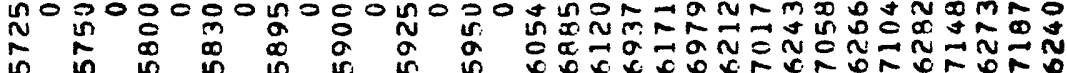
- Uz $000000000 N 000+0000000 m 000000 m 0 m N N N \$ m \alpha 0 N m 00$

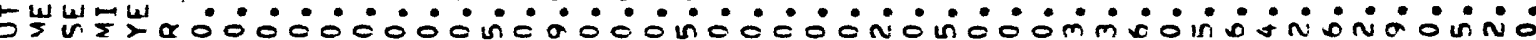
ब, $-\frac{1}{m}$ $\stackrel{\Delta}{\alpha}$ in

ox

움

谣

岁文

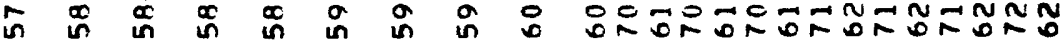

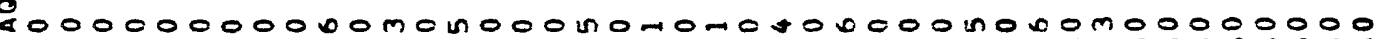

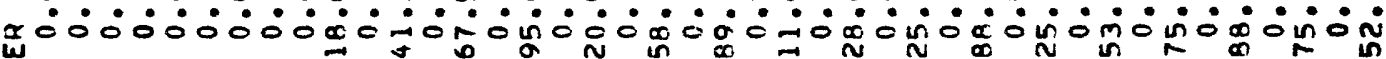

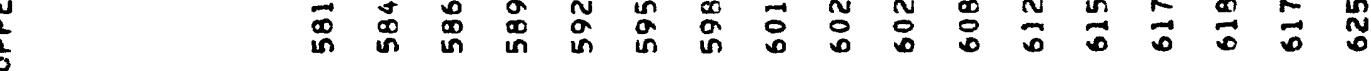


OODO OMOOONONONOOONOMOOO

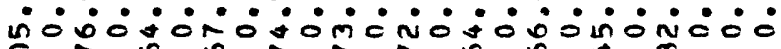

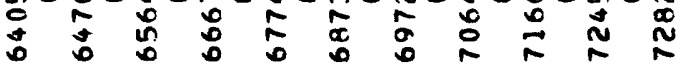

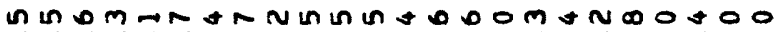
$\dot{0} \dot{0} \dot{0} \dot{0} \dot{0} \dot{0} \dot{0} \dot{0} \dot{0} \dot{0} \dot{0} \dot{0} \dot{0} \dot{0} \dot{0} \dot{0}$

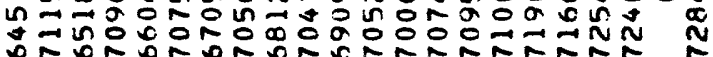

nom n m n n

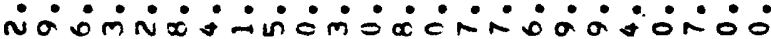

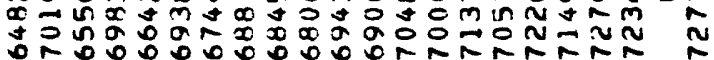

onoaoun unona unoor $\dot{0} \dot{0} \dot{0} \dot{0} \dot{0} \dot{0} \dot{0} \dot{0} \dot{0} \dot{0} \dot{0} \dot{0} \dot{0} \dot{0} \dot{0}$

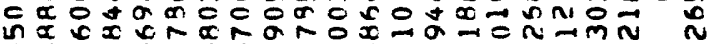

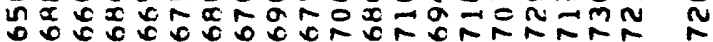

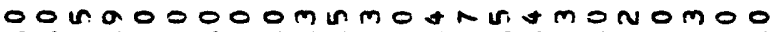
á $\dot{0} \dot{0} \dot{0} \dot{0} \dot{0} \dot{0} \dot{0} \dot{0} \dot{0} \dot{0} \dot{0} \dot{0} \dot{0} \dot{0} \dot{0} \dot{0}$

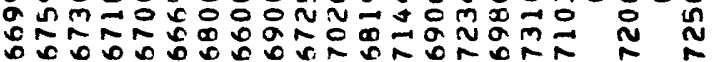

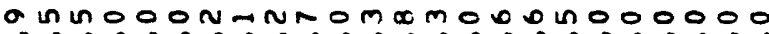
m nojona nó

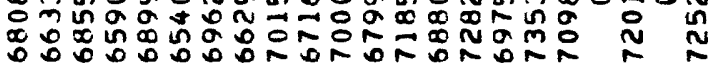

n十nOR nRm

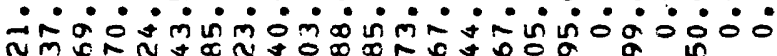

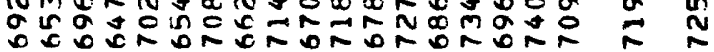

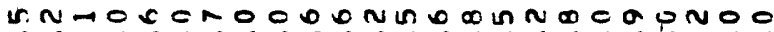

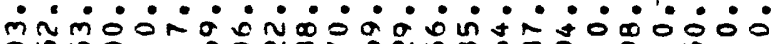
으의

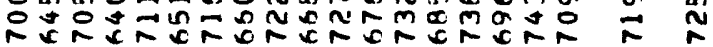

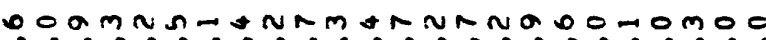
ம் $\dot{0} \dot{0} \dot{0} \dot{0} \dot{m} \dot{m} \dot{m} \dot{0} \dot{0} \dot{0} \dot{0} \dot{0} \dot{0} \dot{0}$

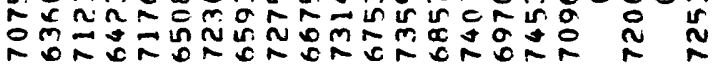

coun unanamm- monotonomono $\dot{0} \dot{0} \dot{0} \dot{0} \dot{0} \dot{n} \dot{0} \dot{0} \dot{0} \dot{0} \dot{0} \dot{0} \dot{0} \dot{0} \dot{0} 0$

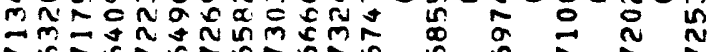

00-0n-arnto000000R000000 $\dot{m} \dot{0} \dot{0} \dot{0} \dot{0} \dot{0} \dot{0} \dot{0} 0 \dot{0} \dot{0} \dot{0} \dot{0} \dot{0} \dot{0} \dot{0}$

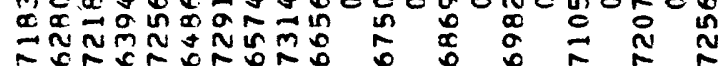

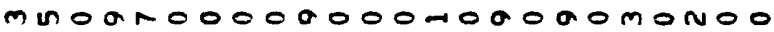

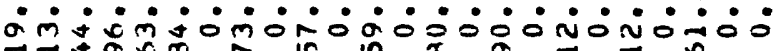

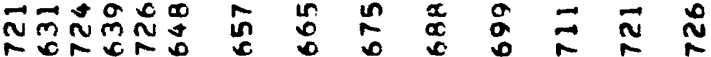

000000007000 UnOm00000ROD

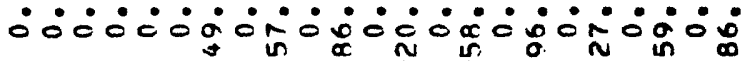

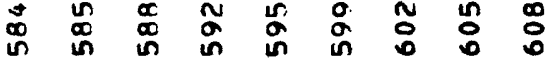

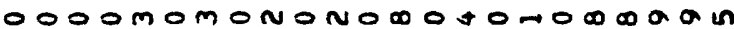
$\dot{0} \dot{0} \dot{0} \dot{0} \dot{0} \dot{0} \dot{0} \dot{0} \dot{0} \dot{0} \dot{0} \dot{0} \dot{N} \dot{0} \dot{m}$

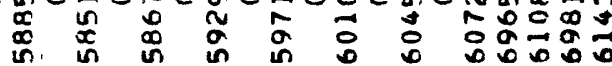

ocoonomoacmonenORORn-40

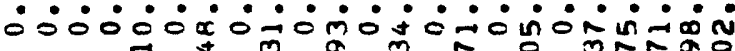
i ${ }^{1}$ in

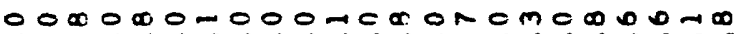
○் $\dot{0} \dot{0} \dot{0} \dot{0} \dot{0} \dot{0} \dot{0} \dot{0} \dot{0} \dot{0} \dot{0} \dot{0} \dot{0}$

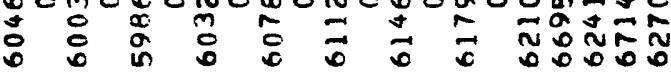

OOMOMOROGODOaONOOOOMmNM

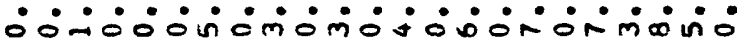

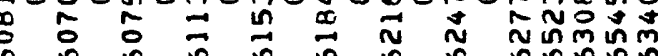

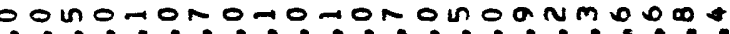

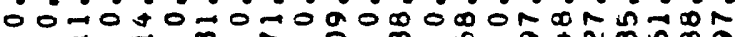

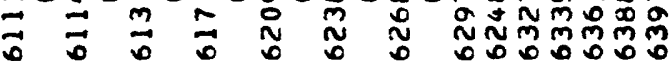

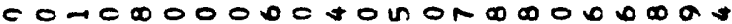
$\dot{0} \dot{0} \dot{0} \dot{0} \dot{0} \dot{0} \dot{0} \dot{0} \dot{0} \dot{0} \dot{0} \dot{0} \dot{0} \dot{0} \dot{0} \dot{m} \dot{0}$ $\frac{m}{0} \underset{0}{*}$ N

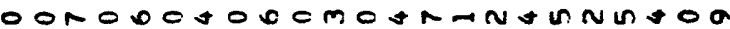
$\dot{0} \dot{0} \dot{0} \dot{0} \dot{0} \dot{0} \dot{0} \dot{0} \dot{0} \dot{0} \dot{m} \dot{m} \dot{0} \dot{0} \dot{0}$

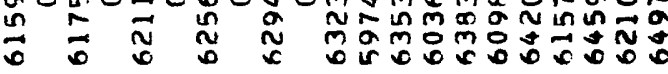

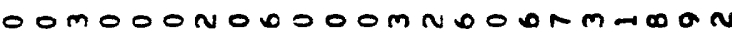

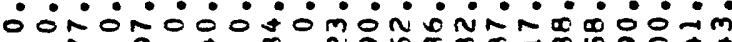

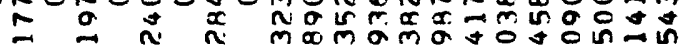
$\overrightarrow{0} \overrightarrow{0}$ ก

$000000000+N M N R \alpha R N 0000$ In $\dot{0} \dot{0} \dot{0} \dot{0} \dot{0} \dot{0} \dot{i} \dot{m} \dot{x} \dot{0} \dot{0} \dot{0} \dot{0} \dot{0} \dot{0} \dot{0}$

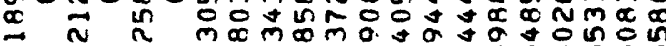
OORONOMONR00*700+00*00\%

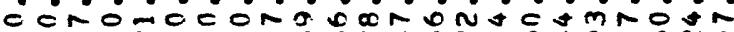
a N N $\vec{b}$ 응 ococoooconononomoaomono

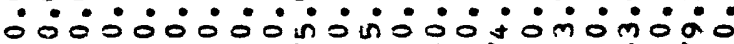

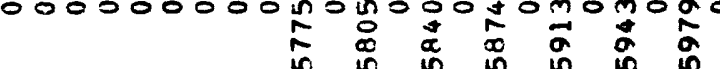

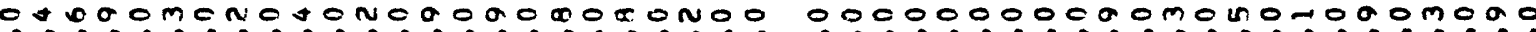

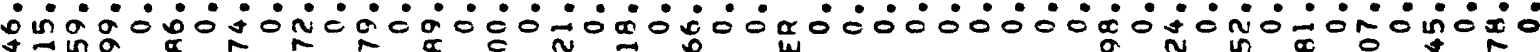

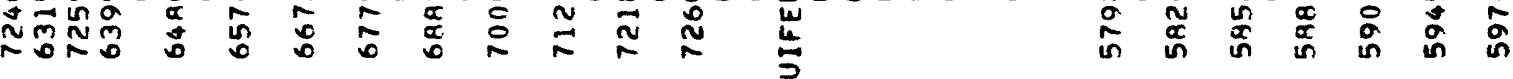

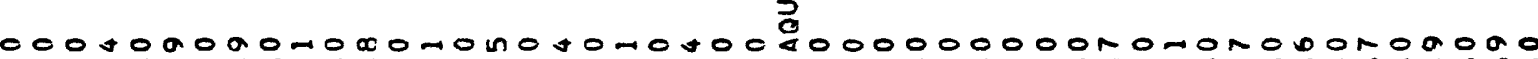

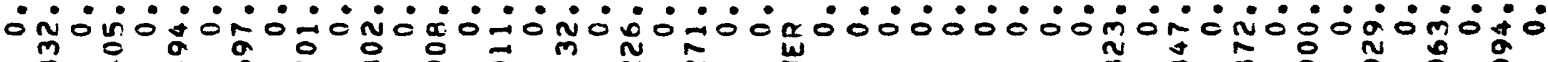

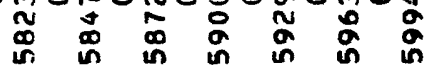




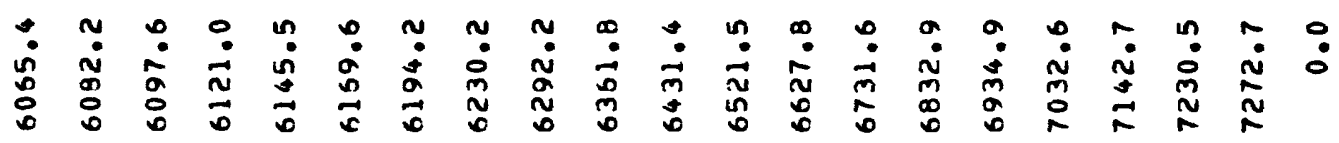

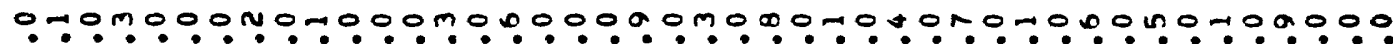

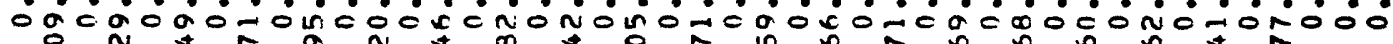

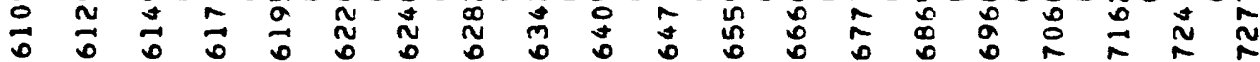

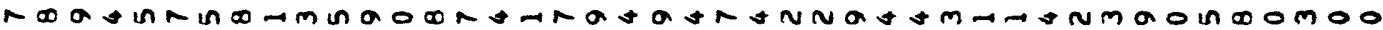

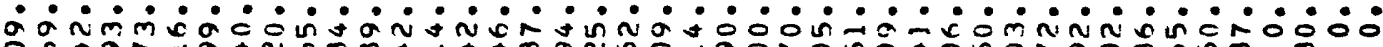

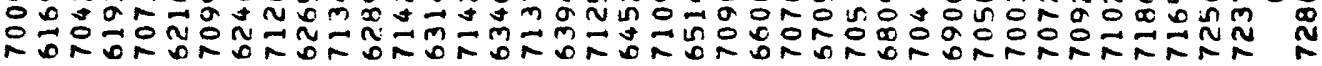

romomranor m m $\dot{0} \dot{0} \dot{0} \dot{0} \dot{0} \dot{0} \dot{0} \dot{0} \dot{0} \dot{0} \dot{0} \dot{0} \dot{0} \dot{0} \dot{0} \dot{0} \dot{0} \dot{0} \dot{0} \dot{0} \dot{0} \dot{0} \dot{0} \dot{0}$

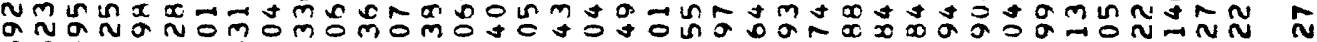

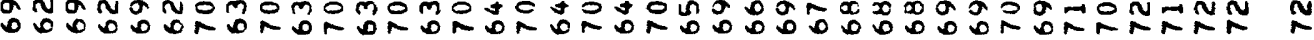

N M m-

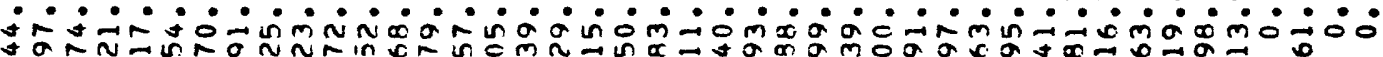

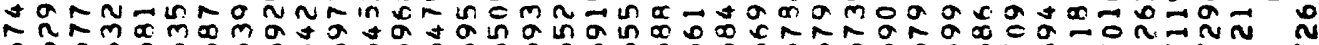

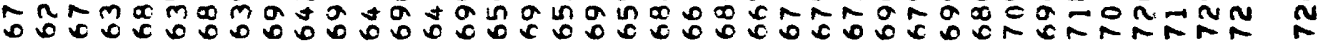

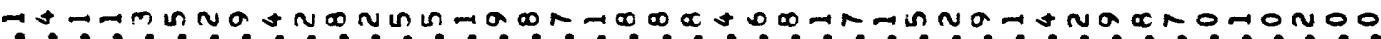

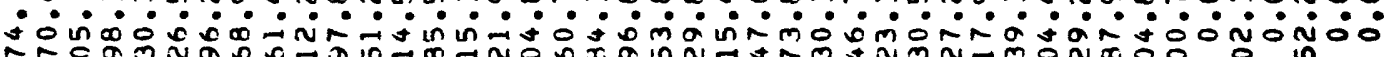

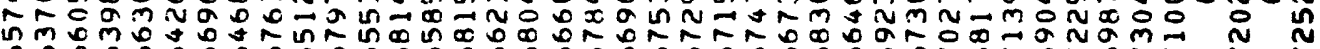

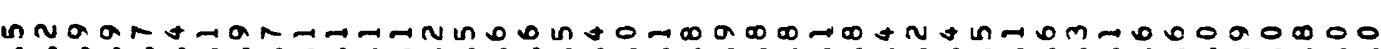

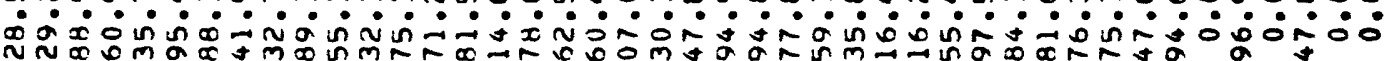

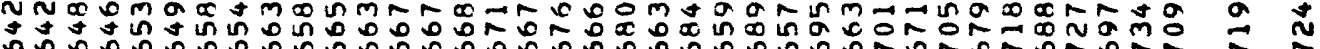

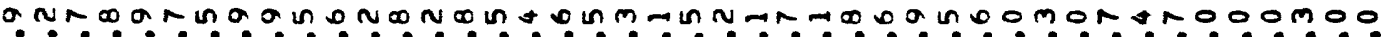

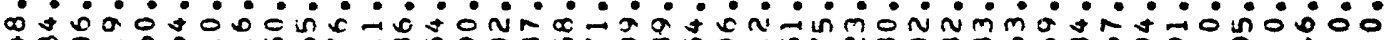

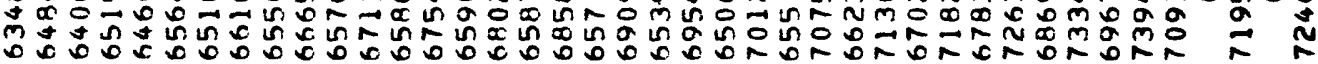

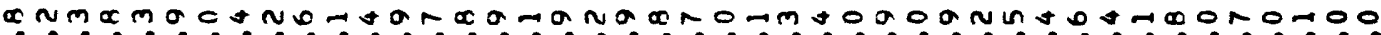

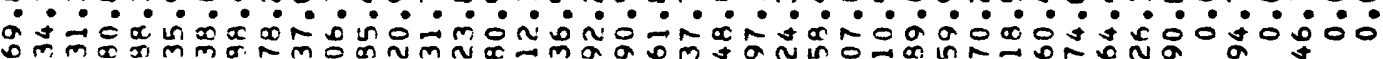

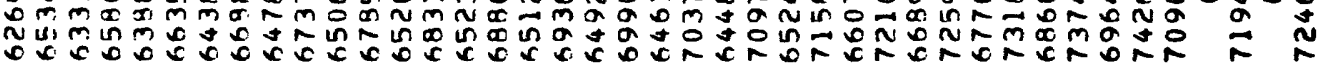

nמmRง

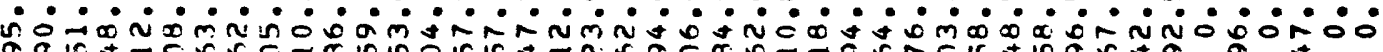

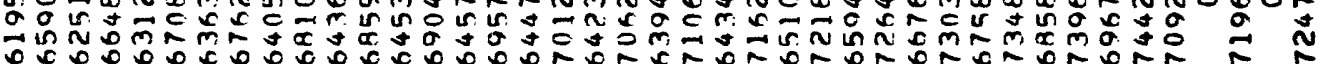

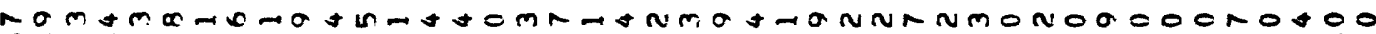
mím

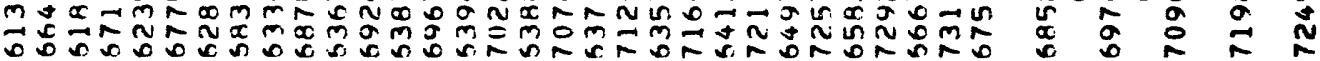

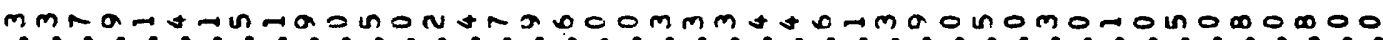

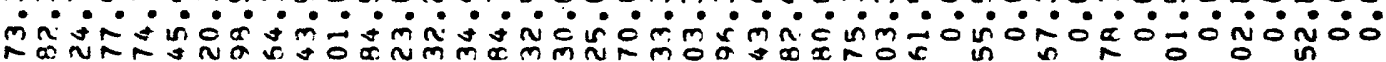

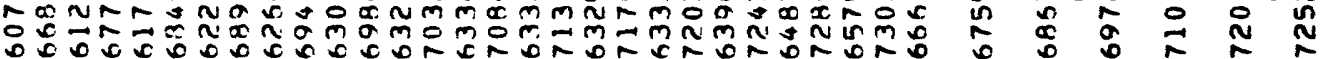

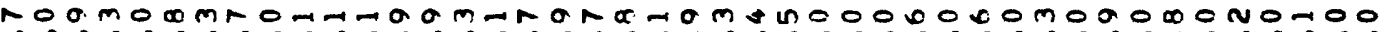

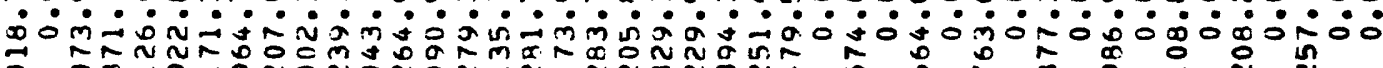

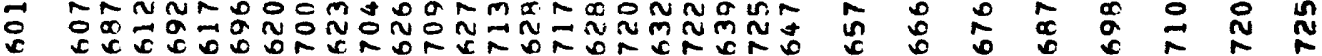

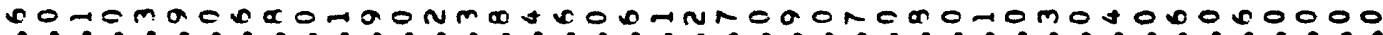

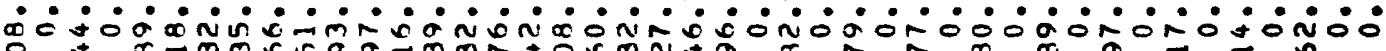

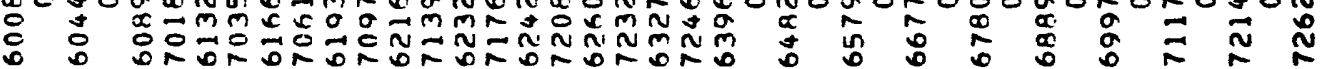

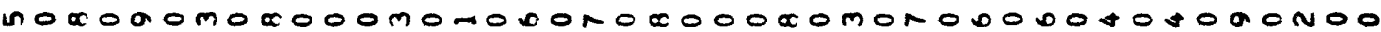
$\therefore \dot{n}$ in og

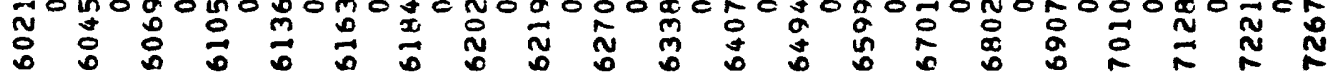




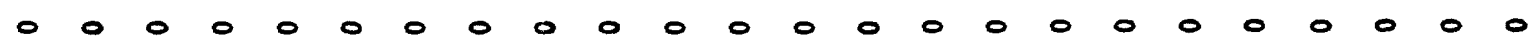

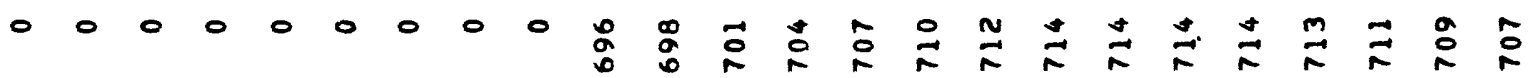

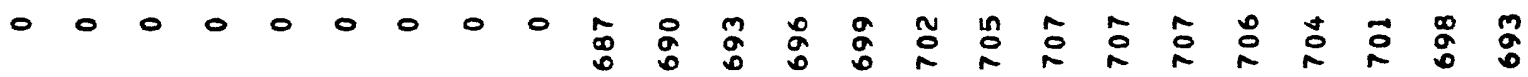

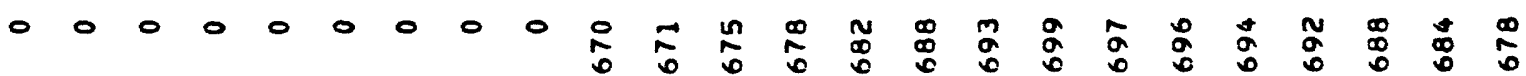

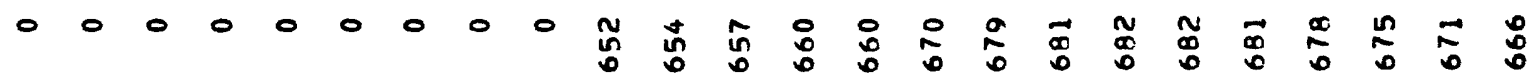

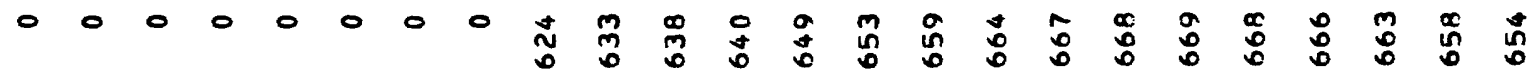

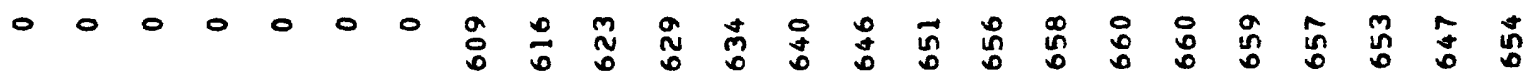

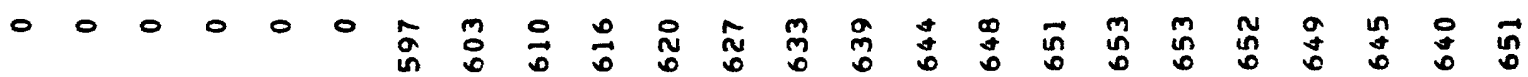

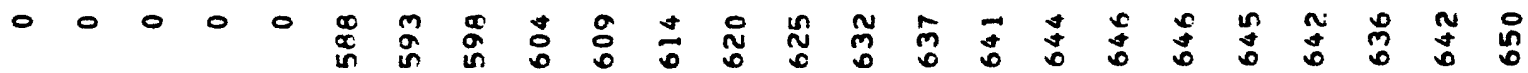

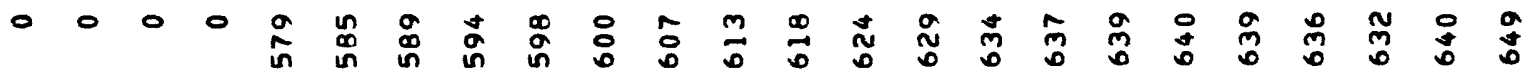

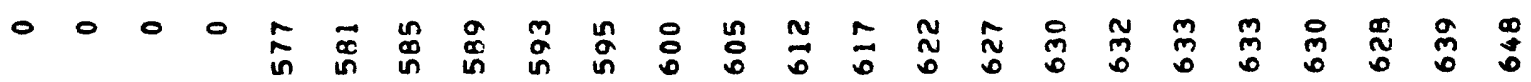

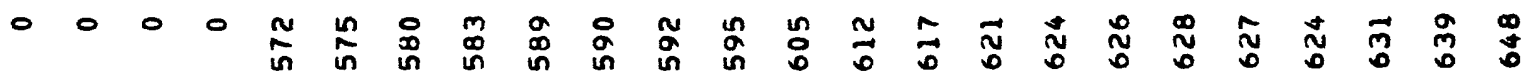

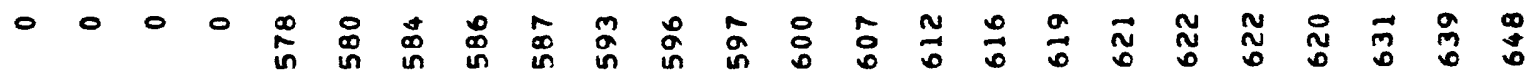

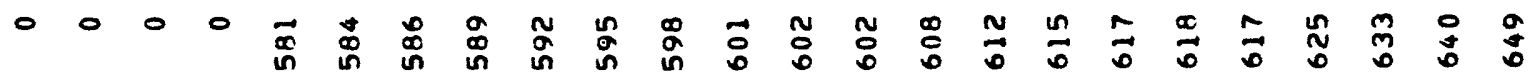

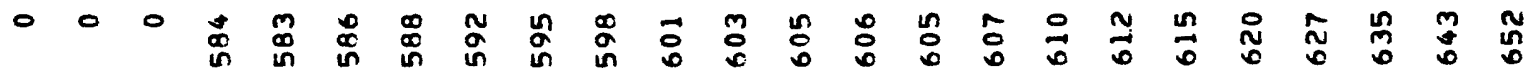

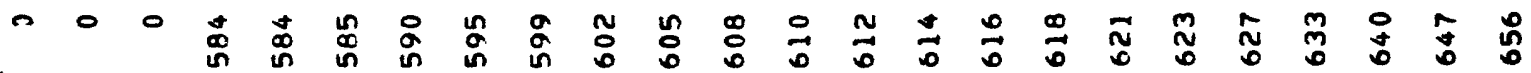

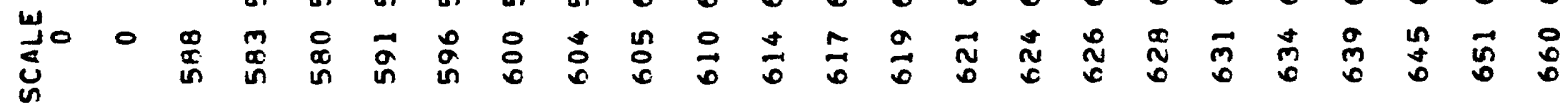

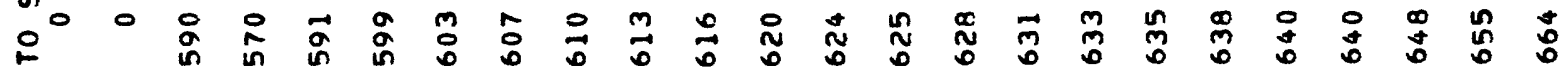

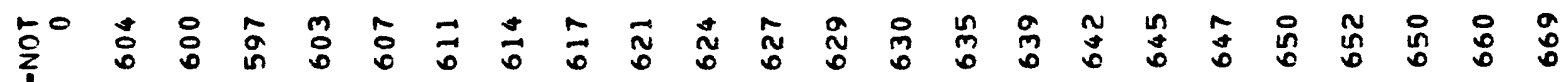

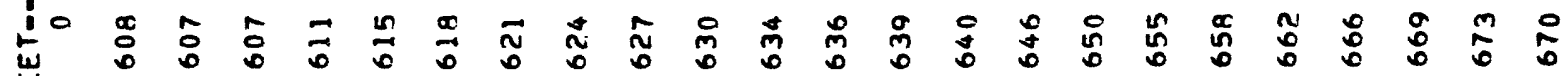

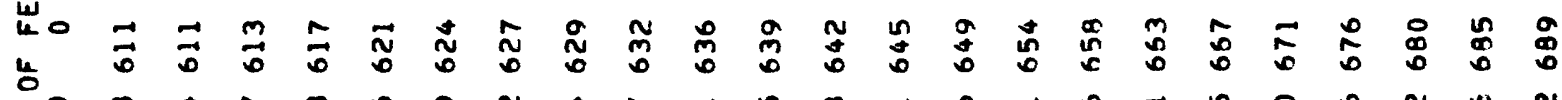

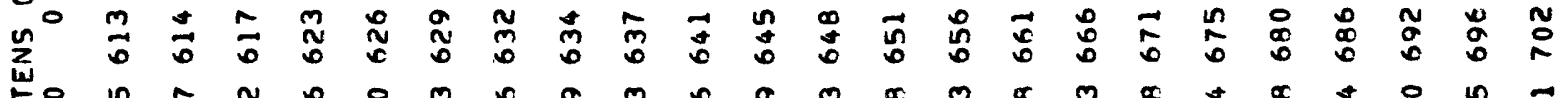

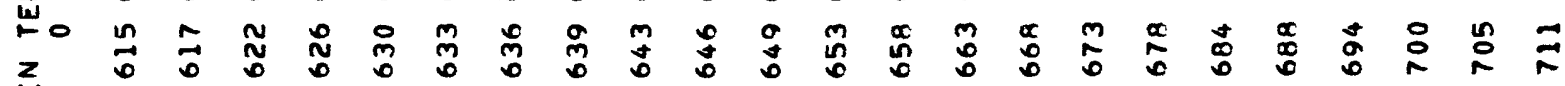

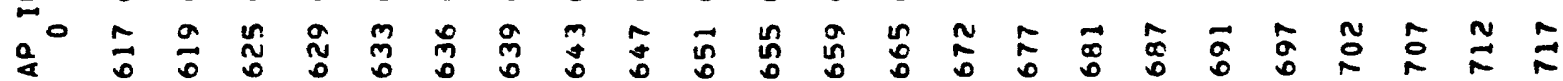

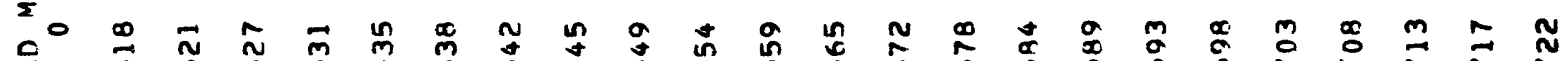

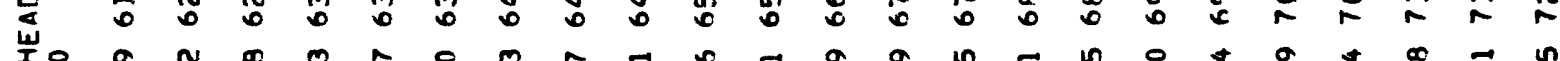

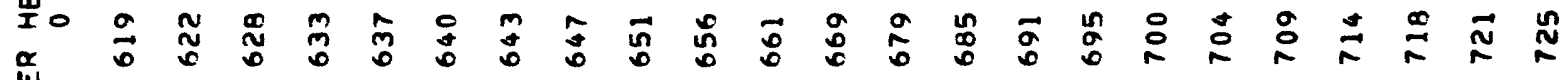

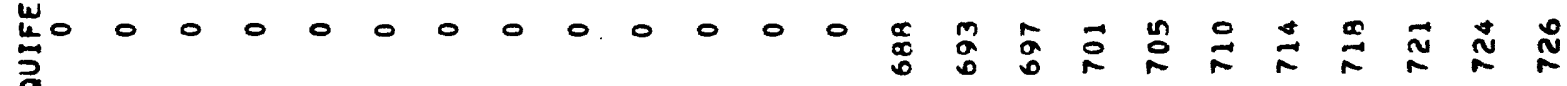

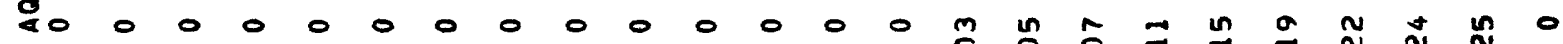


00000000000

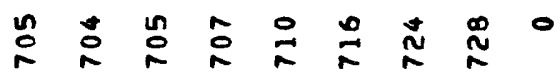

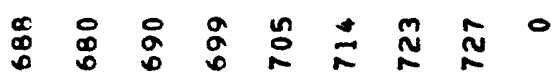

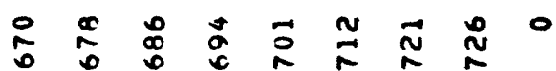

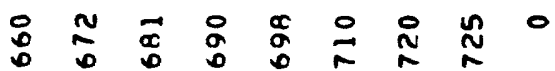

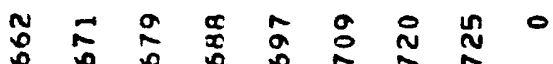

- 00000

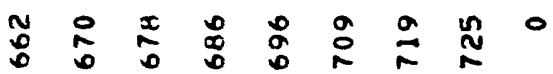

$\begin{array}{lllllllll}0 & \infty & 0 & n & 0 & 0 & 0 & n & 0 \\ 0 & 0 & 0 & 0 & 0 & 0 & \mathbb{N} & 0 & 0\end{array}$

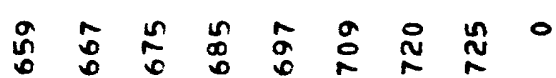

年

in

กิ

ก

貼

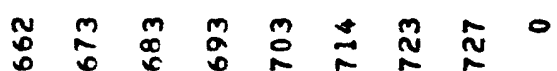

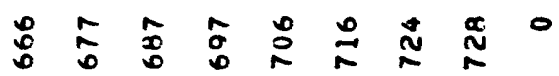

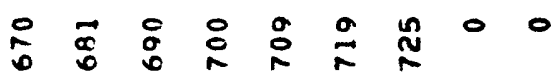

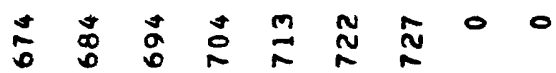

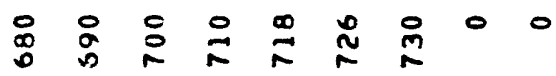

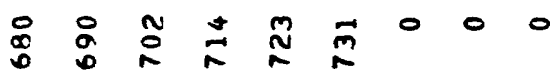

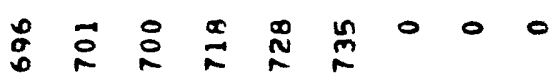

$\stackrel{N}{\infty} \stackrel{N}{N} \stackrel{N}{N} 000$

$\because N \underset{N}{N} \underset{N}{N} \stackrel{m}{N} 000$

$\stackrel{N}{N} \cong \stackrel{M}{N} \stackrel{N}{N} 000$

$\stackrel{N}{N} \underset{N}{N} \underset{N}{N} 000000000$

$\stackrel{N}{N} 0 \quad 0 \quad 0 \quad 00000$

00000000000

000000000000

- 00000000 


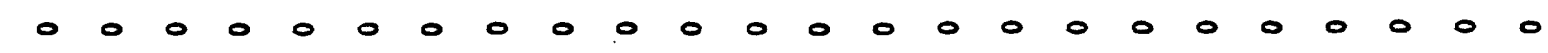

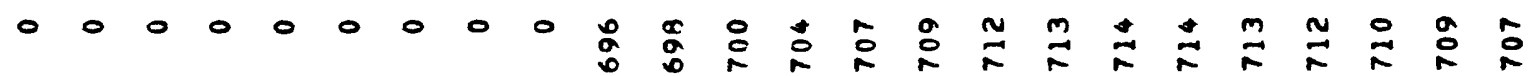

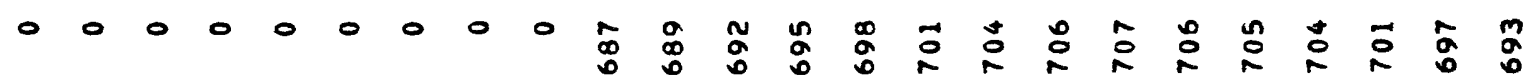

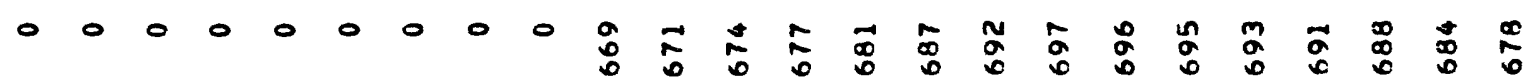

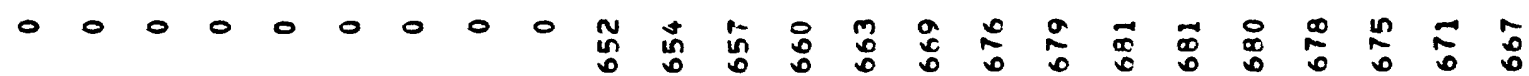

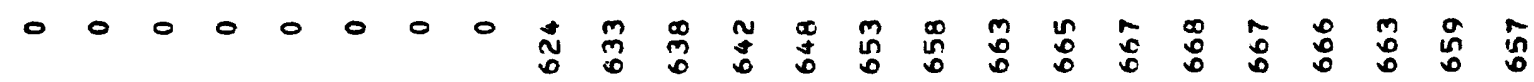

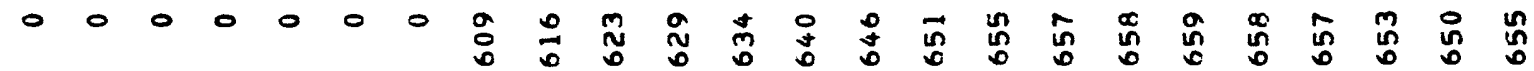

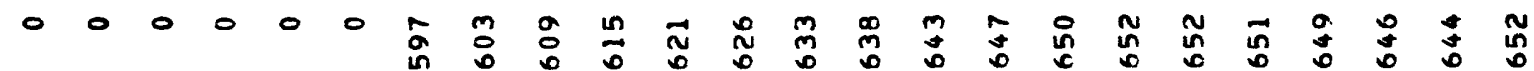

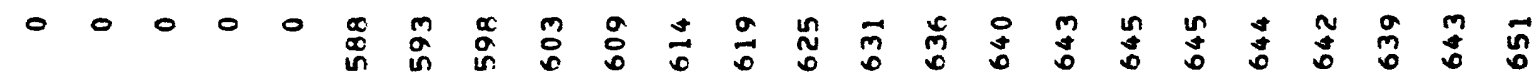

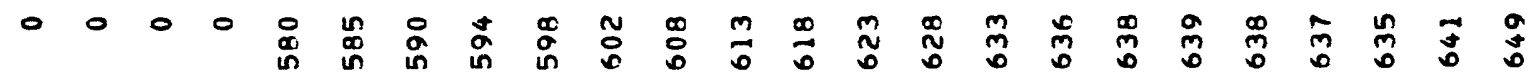

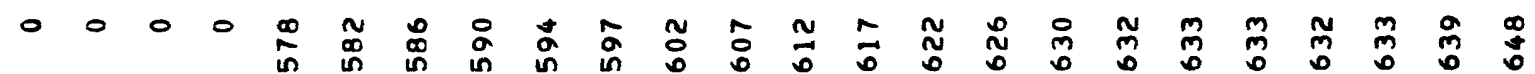

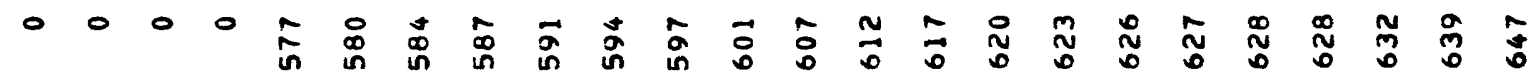

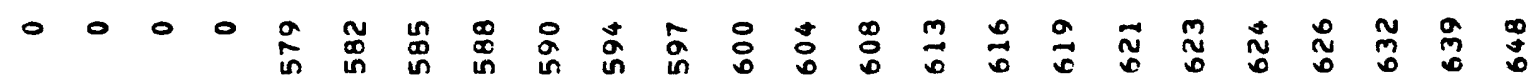

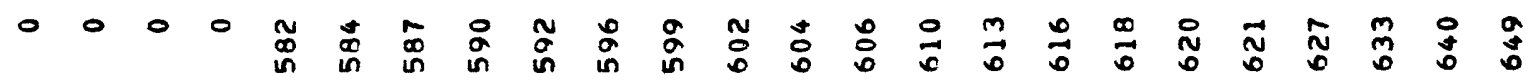

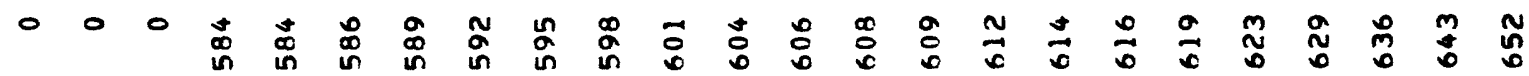

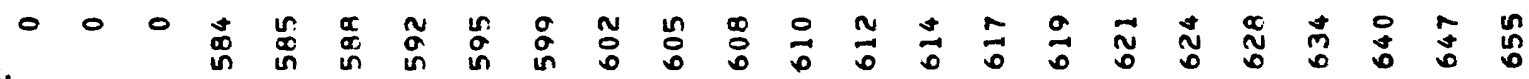

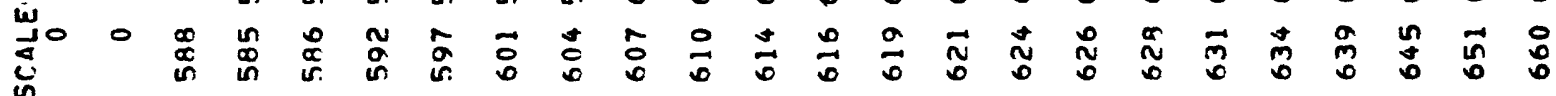

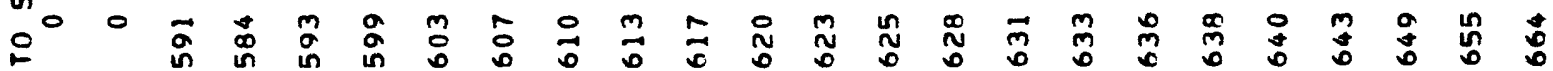

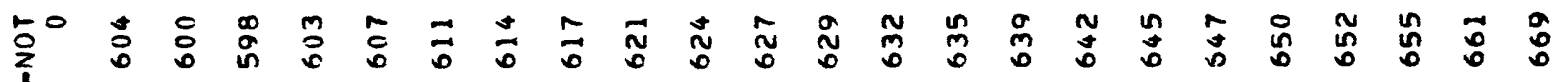

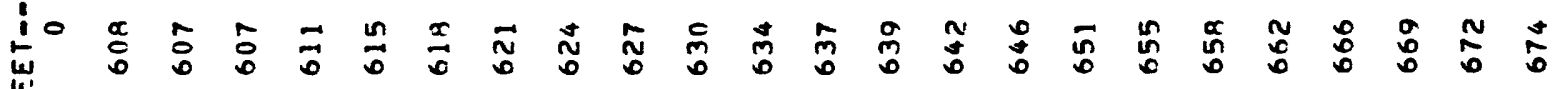

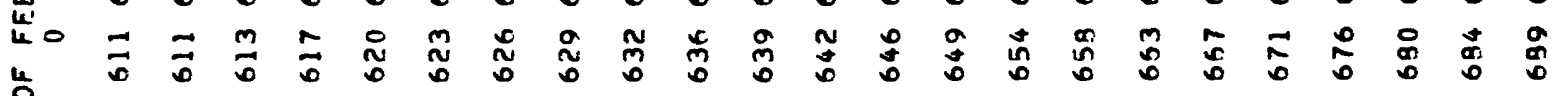

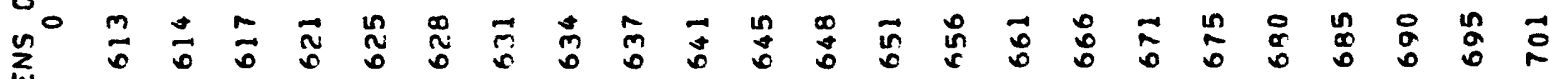

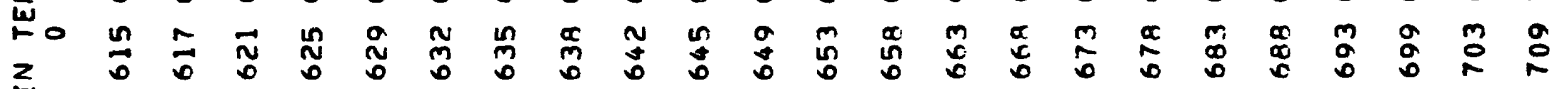

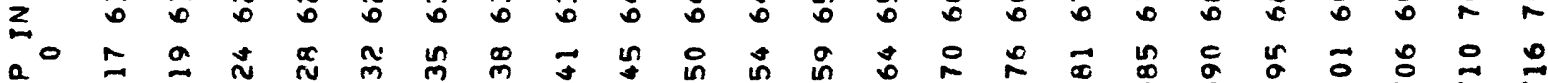

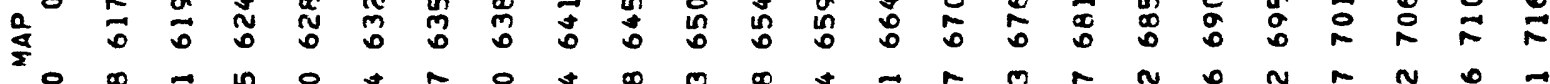

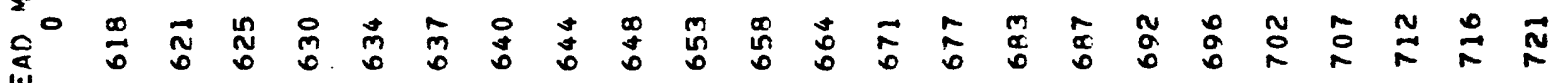

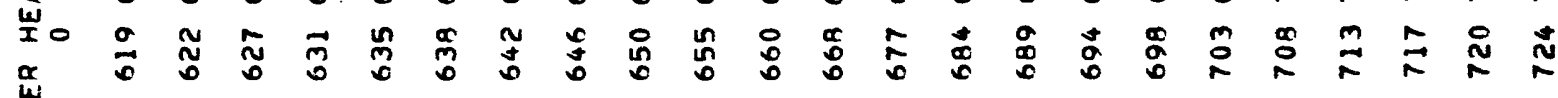

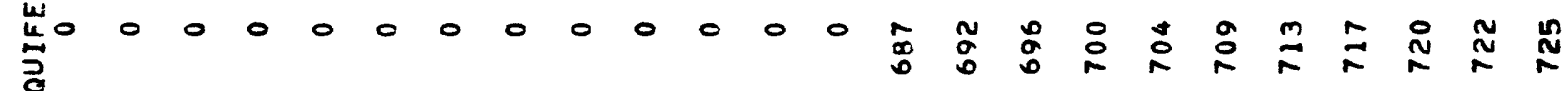

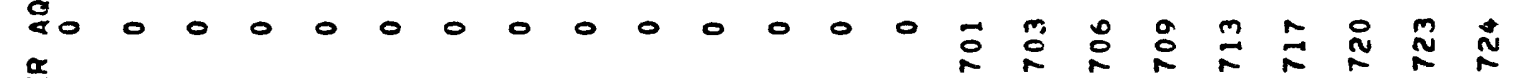

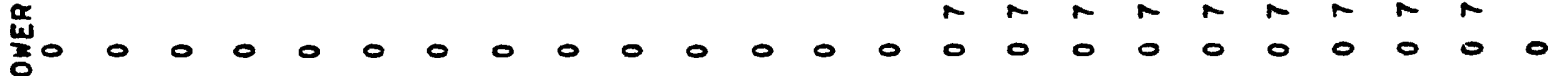


0000000000

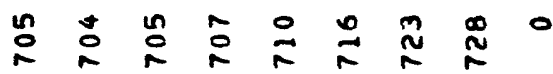

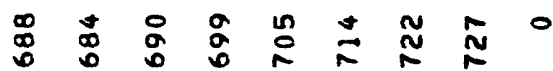

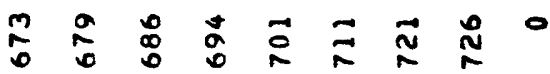

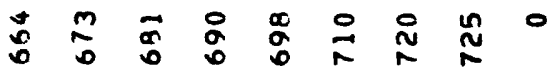

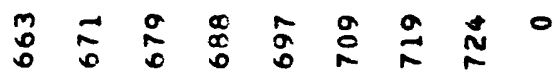

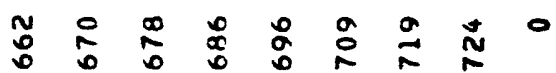

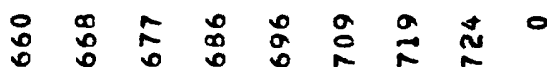

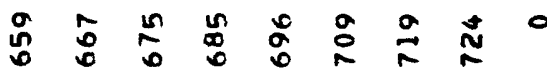

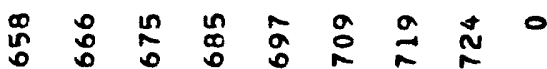

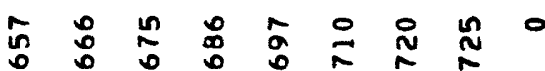

in

ก

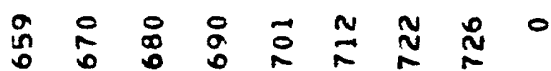

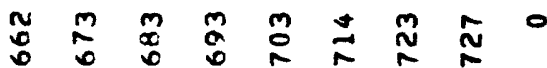

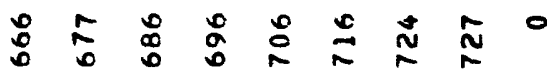

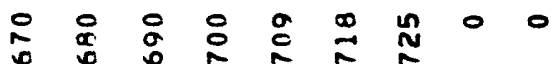

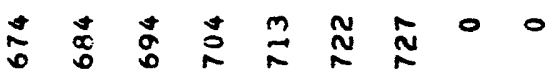

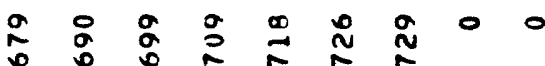

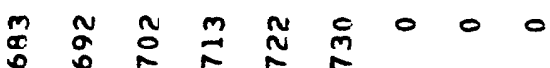

$\stackrel{n}{0} \mathbb{N} \stackrel{N}{N} \stackrel{N}{N} \stackrel{M}{N} 000$

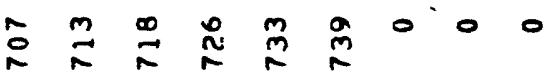

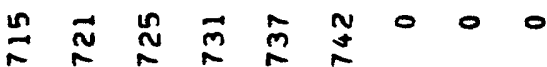

$\mathbb{N} \stackrel{N}{N} \stackrel{M}{M} \stackrel{M}{N} \mathbb{N} \quad 00$

$\stackrel{\sim}{N} \underset{\sim}{M} 0000000$

N 000000000

$\stackrel{M}{N}$

- 00000000

0000000000

000000000 


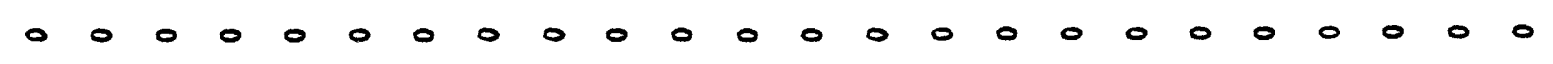

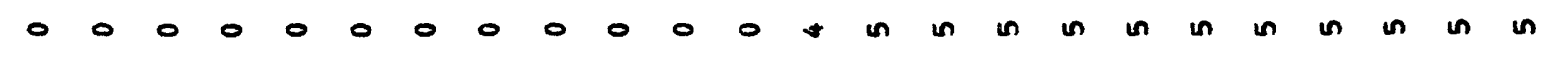

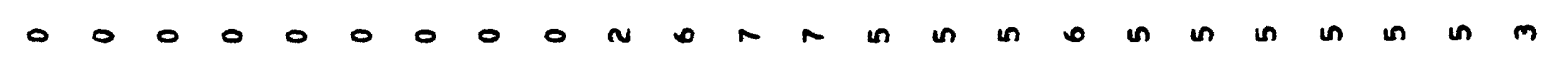

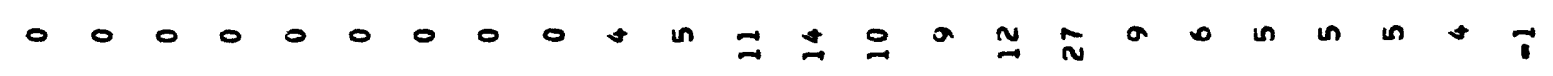

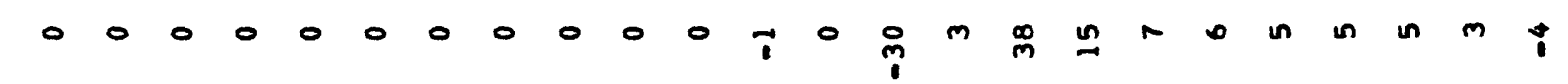

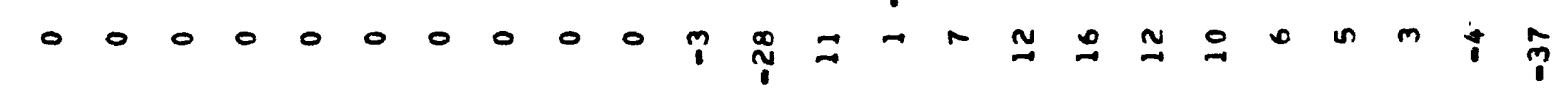

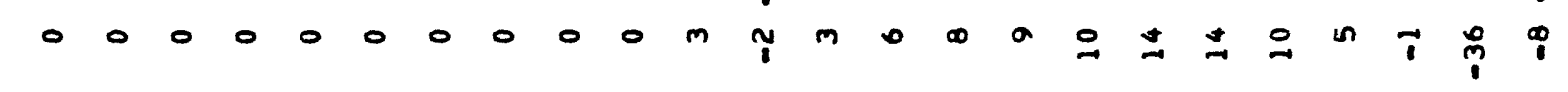

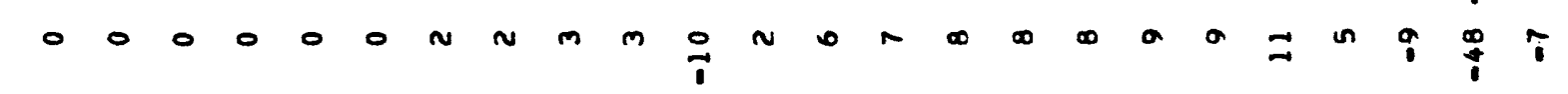

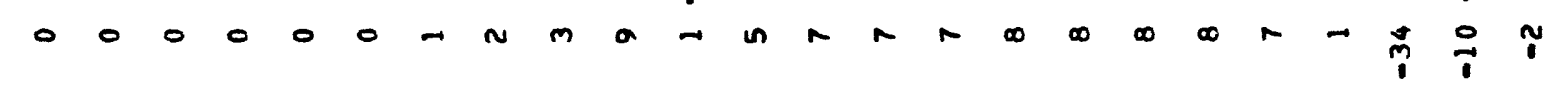
- 0 0 0 M

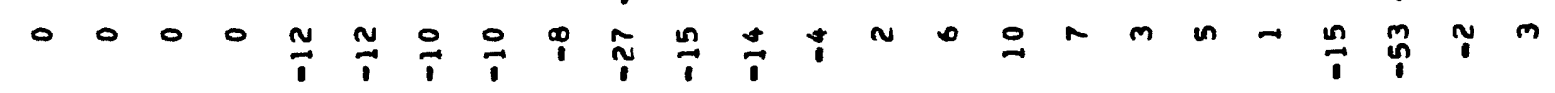
- 0 0 0 i

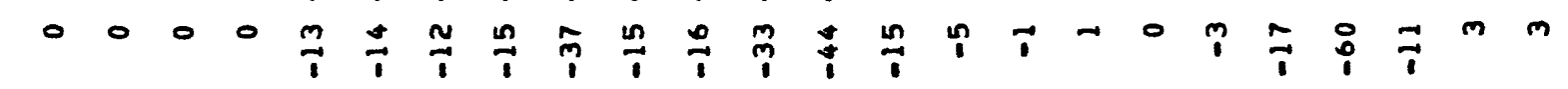
- 0 o 0 n

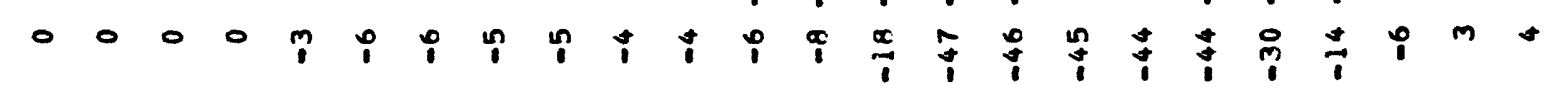
- 0 - $\rightarrow$ m

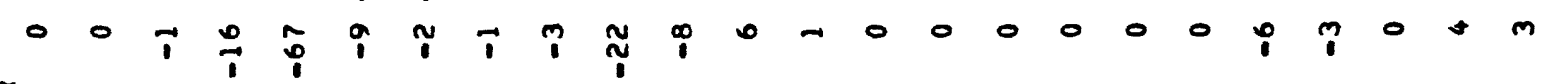

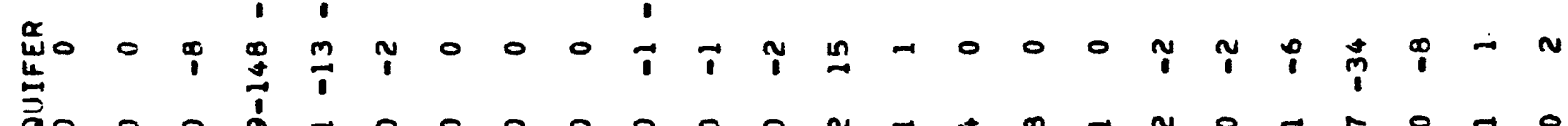
家0 0 0

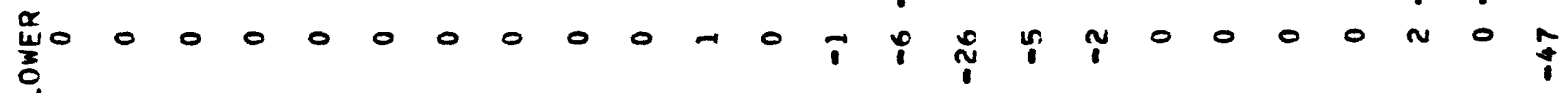

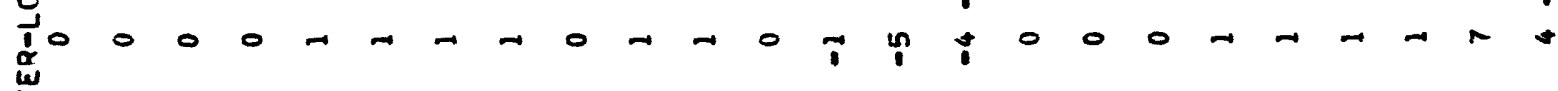

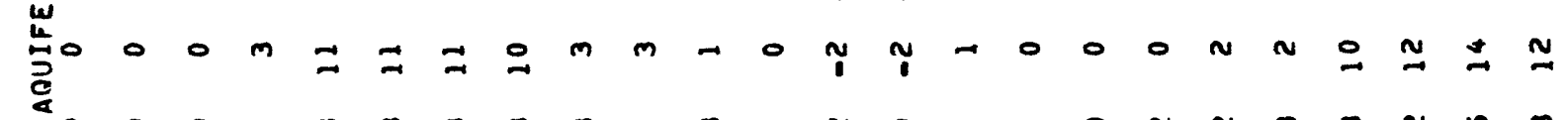

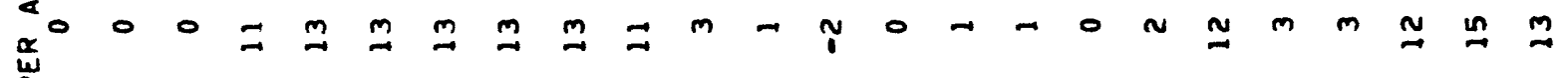

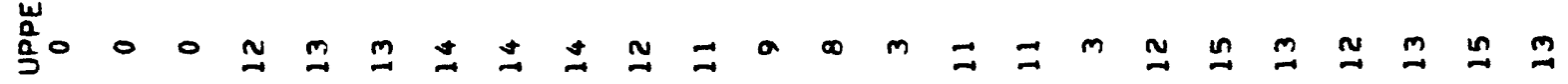

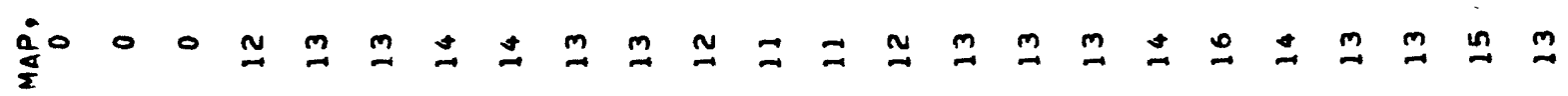

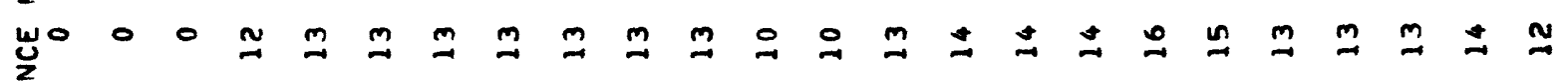

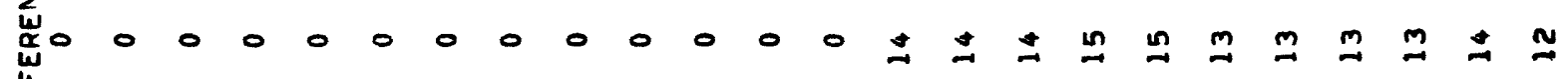

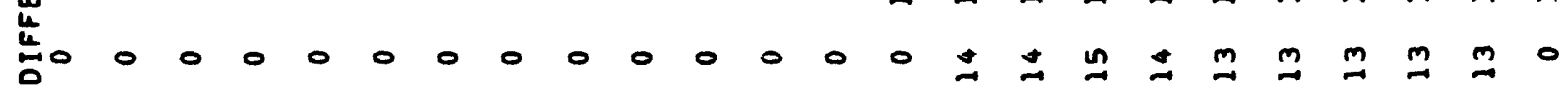
$104 \frac{\omega}{x}$

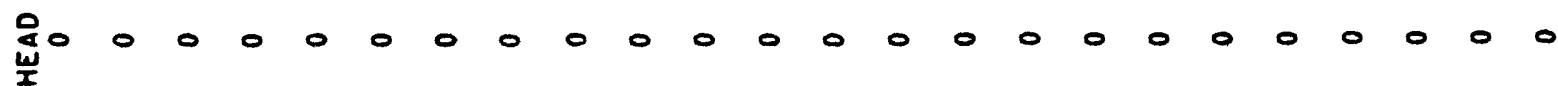




$$
\begin{aligned}
& 00000000000 \\
& +N N \text { N } N-m+0 \\
& \text { m } \$ 000 * 0 \\
& \ddot{m} n \rightarrow N \dddot{i}+* 0 \\
& \text { \& } \\
& \text { i } 0-70 m+t 0 \\
& 00-\text { N } 0 m+\infty \\
& \vec{i} 00 m 0 m+0 \\
& 0 \text { o in } 0 \text { m } *+* 0 \\
& 0 \div ! \div m+*+\infty \\
& \text { o i }
\end{aligned}
$$

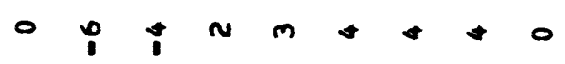

$$
\begin{aligned}
& \text { !n } n 00 m+t \\
& \overrightarrow{1} 000++t+0 \\
& \text { - } 0 m m m+\$ \\
& \circ m m+*+*+0 \\
& \text { - N } m \text { m m m m o } \\
& 000+4 \text { n } 00 \\
& m+n \text { in un n } 00 \\
& m \underset{n}{m} 0+n \text { n } 000 \\
& m \text { n } \\
& \therefore 0 \text { in } 009000 \\
& \Rightarrow=0 \Rightarrow=000 \\
& \Rightarrow \vec{I} \vec{\Rightarrow} \vec{\Rightarrow} 000 \\
& \Rightarrow \ddot{I} 000000 \\
& \Rightarrow \equiv 0000000 \\
& 0000000000 \\
& 00000000000 \\
& 00000000000
\end{aligned}
$$




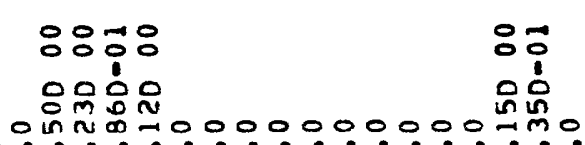

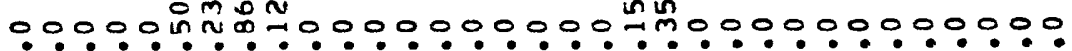

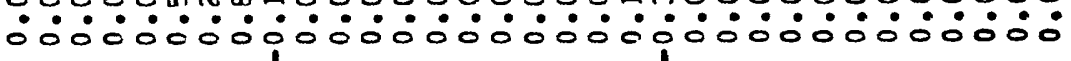

- $\quad 880$

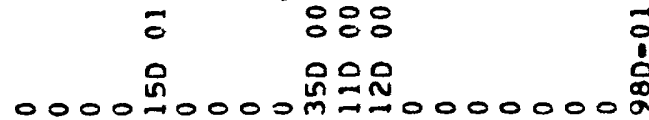

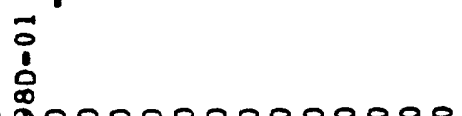

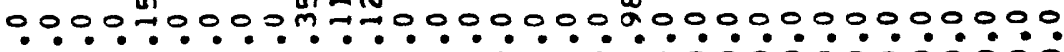

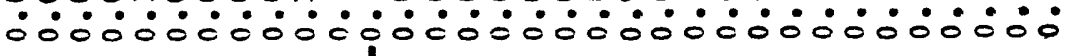
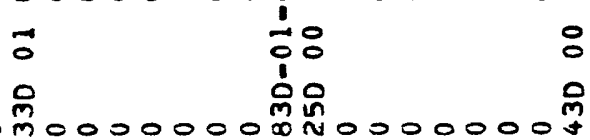

$000 m 0000000 \stackrel{m}{n} 0000000 \stackrel{m}{N} 000000000000$

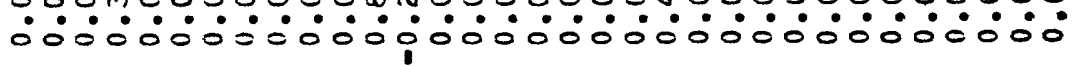

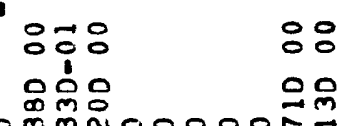

$0000000000000 \mathrm{~m}$ m $00000 \hat{N}=0000000000$

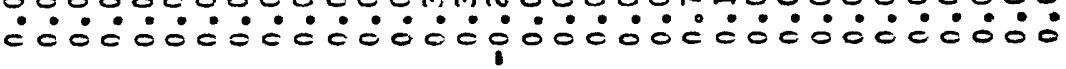

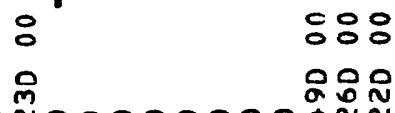

$00000000000000 n 00000000+N N 0000000$

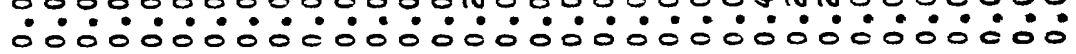
웅 in 00000000000000000000000000 tho00000

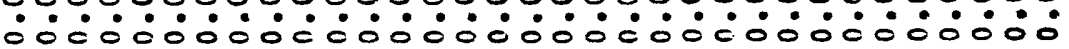

000000000000000000000000000000000

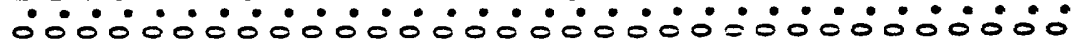

000000000000000000000000000000000

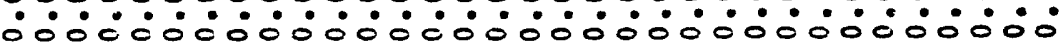

000000000000000000000000000000000

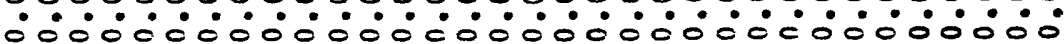

$\approx$

西

$m$

?:

10

H

$\bar{v}$

un

w

함

닝

$\alpha z$

I

$0_{0}^{N}$ $: 0$

0000000000000000000000000000000000

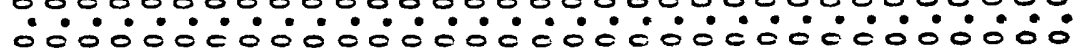

요

赵 000000000000000000000000000000000

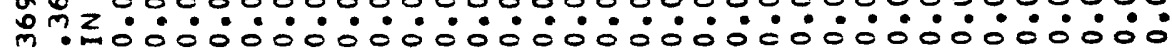

" " $\frac{a}{2}$

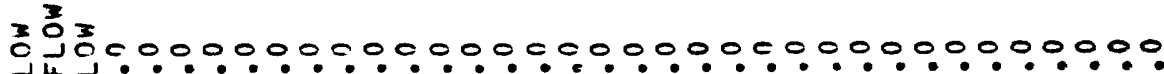
Uا 으은

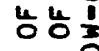

$\omega \omega 000000000000000000000000000000000$

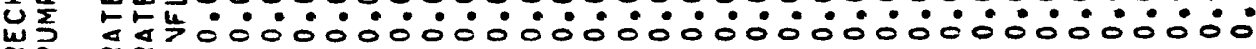


0000000000000000000000000000 N10000

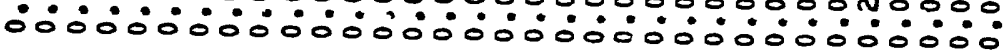

뭉ㅇㅇㅇ

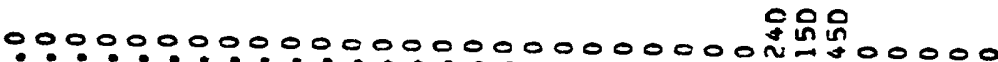

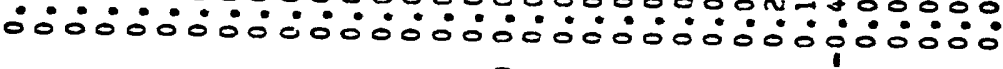

6

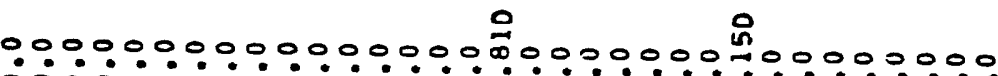
íं जㅇㅇ

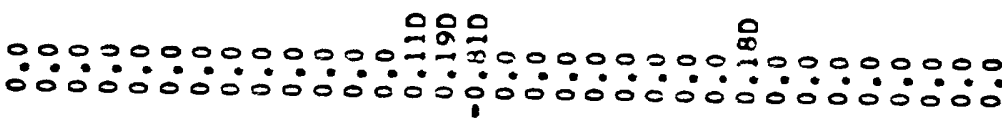
웅용 웅

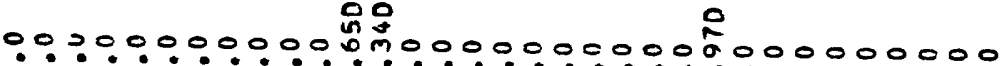

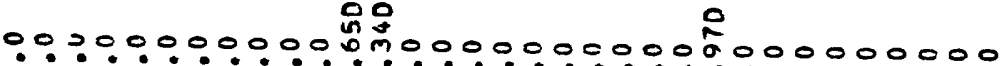
¿́:

\section{8}

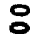

$0003000000 \stackrel{9}{1} 00000000000 \hat{1}$

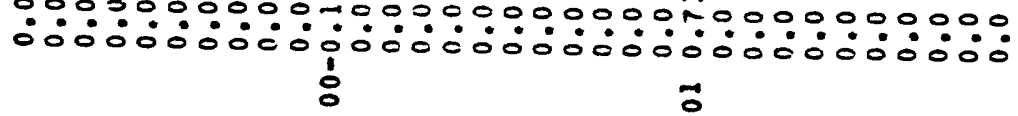

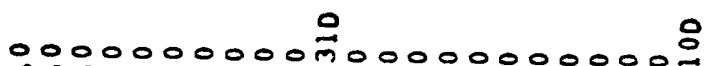

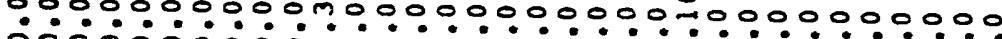

$\begin{array}{ll}0 & 0 \\ 0 & 0\end{array}$

$000000000 \stackrel{m}{n} 00000000000 \pi 00000000000$ 迆00000

:

$000000000^{\infty} 00000000000^{0}$

$\because: 90: \div 0,400000000000 n 00000000000$

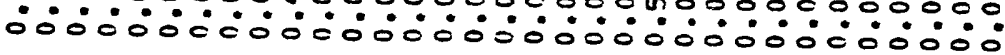

$\begin{array}{ll}8 & 0 \\ 0 & 0\end{array}$

$\because 00000000 \stackrel{0}{100000000000000000000000}$

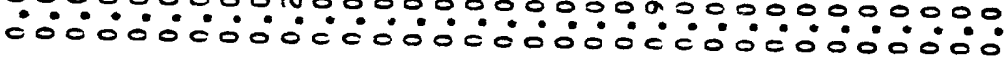

\begin{tabular}{|c|c|}
\hline 등영응 & 용: \\
\hline$-\pi$ & 옹도 \\
\hline
\end{tabular}

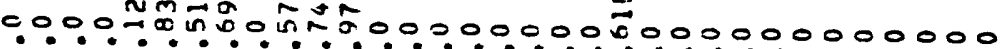

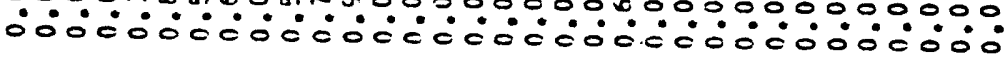

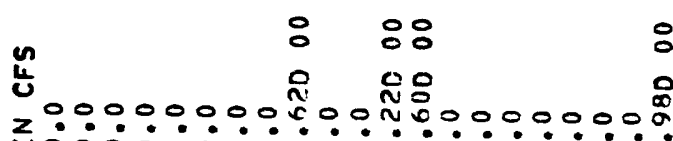

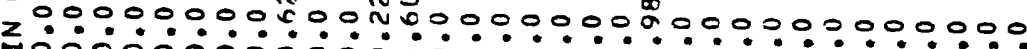
1000000000000000000000000000000000

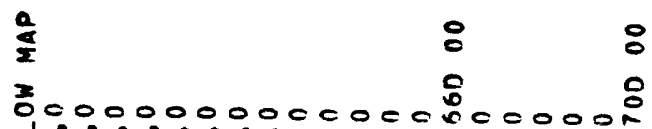

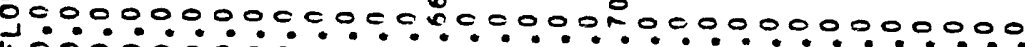

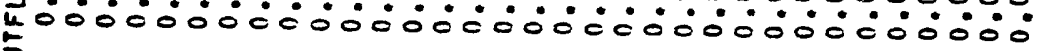
:

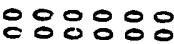
등으으응옹오

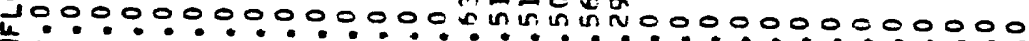

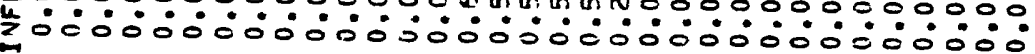




\section{REFERENCES}

Bredehoeft, J. D., and Pinder, G. F., 1970, Digital analysis of areal flow in multiaquifer systems: A quasi three-dimensional model: Water Resources Research, v. 6, no. 3, p. 883-888.

Carnahan, B., Luther, H. A., and Wilkes, J. 0., 1969, Applied numerical methods: New York, John Wiley, $604 \mathrm{p}$.

Coffin, D. L., Welder, F. A., and Glanzman, R. K., 1971, Geohydrology of the Piceance Creek structural basin between the White and Colorado Rivers, northwestern Colorado: U.S. Geological Survey Hydrologic Investigations Atlas $\mathrm{HA}-370$.

Coffin, D. L., Welder, F. A., Glanzman, R. K., and Dutton, X. W., 1968, Geohydrologic data from Piceance Creek basin between the White and Colorado Rivers, northwestern Colorado: Colorado Water Conservation Board Water-Resources Circular 12, $38 \mathrm{p}$.

Donnell, J. R., 1961, Tertiary geology and oil-shale resources of the Piceance Creek bas in between the Colorado and White Rivers, northwestern Colorado: U.S. Geological Survey Bullet in 1082-L, p. 835-891.

Douglas, J., Jr., and Rachford, H. H., Jr., 1956, On the numerical solution of heat conduction problems in two and three space variables: American Mathematical Society Transactions, v. 82, p. 421-439.

Ficke, J. F., Weeks, J. B., and Welder, F. A., 1974, Hydrologic data from the Piceance basin, Colorado: Colorado Water Conservation Board Basic-Data Release $31,246 \mathrm{p}$.

Frickel, D. G., Shown, L. M., and Patton, P. C., 1975, An evaluation of hillslope and channel erosion in the Piceance basin, Colorado: Colorado Water Conservation Board Water-Resources Circular $30,37 \mathrm{p}$.

Trescott, P. C., Pinder, G. F., and Larson, S. P., 1976, Finite-difference model for aquifer simulation in two dimensions with results of numerical experiments: U.S. Geological Survey, Techniques of Water-Resources Investigations, book 7, chapter C1, $116 \mathrm{p}$.

Weeks, J. B., Leavesley, G. H., Welder, F. A., and Saulnier, G. J., Jr., 1974, Simulated effects of oil-shale development on the hydrology of Piceance basin, Colorado: U.S. Geological Survey Professional Paper 908, 84 p.

Weeks, J. B., and Welder, F. A., 1974, Hydrologic and geophysical data from the Piceance basin, Colorado: Colorado Water Conservation Board BasicData Release 35, $121 \mathrm{p}$.

Wymore, I. F., 1974, Estimated average annual water balance for Piceance and Yellow Creek watersheds: Fort Collins, Colorado State University, Environmental Resources Center, Technical Report No. 2, 60 p. 